\title{
Phase separation of biomimetic membranes:
}

\section{Influence of glycosphingolipid structure and substrate adhesion}

\author{
Dissertation \\ for the award of the degree \\ Doctor rerum naturalium \\ of the Georg-August University Göttingen \\ within the doctoral program \\ Chemistry of the Georg-August University School of Science (GAUSS)
}

submitted by

Jeremias Sibold

from Donaueschingen

Göttingen 2019 
Members of the Thesis Committee:

Prof. Dr. Claudia Steinem (Reviewer),

Institute of Organic and Biomolecular Chemistry,

Georg-August University Göttingen

Prof. Dr. Jörg Enderlein,

Third Institute of Physics,

Georg-August University Göttingen

Members of the Examination Board:

Prof. Dr. Claudia Steinem (Reviewer),

Institute of Organic and Biomolecular Chemistry,

Georg-August University Göttingen

Prof. Dr. Jörg Enderlein,

Third Institute of Physics,

Georg-August University Göttingen

Further members of the Examination Board:

apl. Prof. Dr. Burkhard Geil,

Institute of Physical Chemistry,

Georg-August University Göttingen

Prof. Dr. Marcus Müller,

Institute for Theoretical Physics,

Georg-August University Göttingen

apl. Prof. Dr. Bert L. de Groot, Institute for Computational Biolmolecular Dynamics,

Max Planck Institute for Biophysical Chemistry

Prof. Dr. Sarah Köster,

Institute for X-Ray Physics,

Georg-August University Göttingen

Date of oral examination:

14.10.2019 


\section{Declaration}

I, Jeremias Sibold, hereby certify, that this thesis has been written independently and there was no use of other sources than quoted.

Göttingen, 2019

Jeremias Sibold 

- Meiner Familie -

„Verzicht auf Denken ist geistige Bankrotterklärung."

Albert Schweizer 



\section{Abstract}

The bacterium enterohemorrhagic Escherichia coli (EHEC) is one of the main causes of food borne illness. The toxin of this bacteria is shiga toxin (STx) which inhibits the protein biosynthesis of cells. Approaching the cells B-subunit of STx (STxB) binds to the receptor lipid globotriaosyl ceramide $\left(\mathrm{Gb}_{3}\right)$ which is heterogeneous distributed in the outer membrane of human cells. Such a cellular membrane is itself very heterogeneous and has regions of higher and lower ordering which influences $\mathrm{Gb}_{3}$ distribution. Furthermore, the cellular membrane is heterogeneously connected either to the cytoskeleton in the inside or to the surfaces on the outside of the cell, inducing different adhesional properties in the cell membrane (adhered membranes and non-adhered membranes). As postulated in a theory of Lipowsky et al. 1 this is assumed to influence ordering and lipid distribution in lipid membranes.

In this work, the phase distribution of the $\mathrm{Gb}_{3}$ is analyzed in biomimetic model membranes as a function of the creamide backbone harboring different fatty acids.

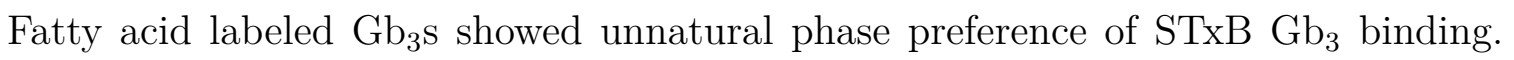
Fluorescently head group labeled $\mathrm{Gb}_{3} \mathrm{~s}$ were investigated to study the influence of different fatty acids in liquid ordered $\left(l_{\mathrm{o}}\right)$ /liquid disordered $\left(l_{\mathrm{d}}\right)$ phase-separated giant unilamellar vesicles. $\mathrm{Gb}_{3} \mathrm{~S}$ with the saturated fatty acid is preferentially distributed in the $l_{\mathrm{o}}$ phase, while the $\mathrm{Gb}_{3} \mathrm{~s}$ with the unsaturated fatty acid prefer the $l_{\mathrm{d}}$ phase. An $\alpha$-hydroxylation at the fatty acid had no effect of the distribution of $\mathrm{Gb}_{3}$. In contrast different sphingomyelins ( $\mathrm{SMs}$ ) influence the partitioning of $\mathrm{Gb}_{3}$.

The influence of heterogeneous membrane adhesion to biomimic membranes was analyzed using pore-spanning membranes (PSMs) on $\mathrm{SiO}_{\mathrm{x}}$ functionalized substrates. The heterogeneous membrane adhesion influences the measured phase diagram. A membrane composed of DOPC/SM/cholesterol (Chol) (1:1:2) is still phase-separated with the $l_{\mathrm{d}}$ phase in the solid supported PSM (s-PSM) and the $l_{\mathrm{o}}$ phase in the freestanding PSM (f-PSM). Phase diagrams of homogeneous membrane adhesion were reported to have no phase separation at the same Chol content which verifies the theory of Lipowsky et al.1 1 for the analyzed lipid compositions. 



\section{Abbreviations}

${ }^{2} \mathrm{H}-\mathrm{NMR}$

AFM

ATTO488-DOPE

ATTO488-DPPE

ATTO532-DOPE

ATTO647N-DOPE

ATTO655-DOPE

BODIPY

BODIPY 500/510-PC

BODIPY-Chol

BODIPY-PC

BSA

cb-DOPE

Chol

CLSM

CPEO3

CTx

CTxB

CVE

Cy3

$D$

$\mathrm{DiD}_{18}$

DiI-C $_{18}$

DLPC deuterium nuclear magnetic resonance

atomic force microscopy

ATTO488-1,2-dioleoyl-sn-glycero-3-phosphoethanolamin

ATTO488-1,2-dipalmitoyl-sn-glycero-3-

phosphoethanolamin

ATTO532-1,2-dioleoyl-sn-glycero-3-phosphoethanolamin

ATTO647N-1,2-dioleoyl-sn-glycero-3-phosphoethanolamin

ATTO655-1,2-dioleoyl-sn-glycero-3-phosphoethanolamin boron-dipyrromethene

2-(4,4-difluoro-5-octyl-4-bora-3a,4a-diaza-s-indacene-3pentanoyl)-1-hexadecanoyl-sn-glycero-3-phosphocholine 23-(dipyrrometheneboron difluoride)-24-norcholesterol 2-(4,4-difluoro-5,7-dimethyl-4-bora-3a,4adiaza-s-indacene-3pentanoyl)-1-hexadecanoyl-sn-glycero-3-phosphocholine bovine serum albumin 1,2-dioleoyl-sn-glycero-3-phosphoethanolamine-N-capbiotinyl

cholesterol

confocal laser scanning microscope

$O$-cholesteryl $\mathrm{N}$-(8'-mercapto-3',6'-dioxaoctyl)carbamate

cholera toxin

B-subunit of cholera toxin

covariance-based estimation

cyanine dye 3

diffusion coefficient

Dioctadecyl-3,3,3',3'-tetramethylindodicarbocyanine perchlorate

1,1'dioctadecyl-3,3,3',3'-tetramethylindocarbocyanine perchlorate

1,2-dilauroyl-sn-glycero-3-phosphatidylcholine 
DMPC

DOPC

DOPE

DPhPC

DPPC

DPPE

DPPE-KK114

DRM

DSC

DSPE

DSPE-KK114

DSPE-PEG-KK114

Dy731-DOPE

EHEC

FCS

f-PSM

FRAP

FRET

GalCer

$\mathrm{Gb}_{3} \mathrm{PH}$

$\mathrm{Gb}_{3 \text { porc }}$

$\mathrm{Gb}_{3} \mathrm{TT}$

$\mathrm{Gb}_{3}$

$\mathrm{Gb}_{3} \mathrm{PEG}_{13} \mathrm{C}_{24: 0} \mathrm{H}$

$\mathrm{Gb}_{3} \mathrm{PEG}_{13} \mathrm{C}_{24: 0} \mathrm{OH}$

$\mathrm{Gb}_{3} \mathrm{PEG}_{13} \mathrm{C}_{24: 1} \mathrm{H}$

$\mathrm{Gb}_{3} \mathrm{PEG}_{13} \mathrm{C}_{24: 1} \mathrm{OH}$

$\mathrm{Gb}_{3} \mathrm{PEG}_{3} \mathrm{C}_{24: 0} \mathrm{H}$

$\mathrm{Gb}_{3} \mathrm{PEG}_{3} \mathrm{C}_{24: 0} \mathrm{OH}$ 1,2-dimiristeoyl-sn-glycero-3-phosphatidylcholine

1,2-dioleoyl-sn-glycero-3-phosphatidylcholine

1,2-dioleoyl-sn-glycero-3-phosphatidylethanolamine

1,2-diphytanoyl-sn-glycero-3-phosphatidylcholine

1,2-dipalmitoyl-sn-glycero-3-phosphatidylcholine

1,2-dipalmitoyl-sn-glycero-3-phosphatidylethanolamine

KK114-1,2-dipalmitoyl-sn-glycero-3-phosphoethanolamin detergent restistant membrane

differential scanning calorimetry

1,2-stearoyl-sn-glycero-3-phosphatidylethanolamine

KK114-1,2-distearoyl-sn-glycero-3-phosphoethanolamin

KK114-PEG ${ }_{45}$-1,2-distearoyl-sn-glycero-3-phosphoethanolamin

Dy731-PEG ${ }_{25}$-1,2-dioleoyl-sn-glycero-3-phosphoethanolamin

enterohemorrhagic Escherichia coli

fluorescence correlation spectroscopy

freestanding pore-spanning membrane

fluorescence recovery after photobleaching

fluorescence resonance energy transfer

galactosylceramide

globotriaosyl ceramid phenyl-modified fatty acid

porcine erythrocytes globotriaosyl ceramide

globotriaosyl ceramid thienothienyl-modified fatty acid

globotriaosyl ceramide

BODIPY labeled lignoceroyl globotriaosyl ceramid with 13 PEG units

BODIPY labeled 2-R-hydroxyl-lignoceroyl globotriaosyl ceramid with 13 PEG units

BODIPY labeled lignoceroyl nervonoyl ceramid with 13 PEG units

BODIPY labeled 2-R-hydroxyl-nervonoyl globotriaosyl ceramid with 13 PEG units

BODIPY labeled lignoceroyl globotriaosyl ceramid with 3 PEG units

BODIPY labeled 2-R-hydroxyl-lignoceroyl globotriaosyl ceramid with 3 PEG units 


\begin{tabular}{|c|c|}
\hline $\mathrm{Gb}_{3} \mathrm{PEG}_{3} \mathrm{C}_{24: 1} \mathrm{H}$ & $\begin{array}{l}\text { BODIPY labeled lignoceroyl nervonoyl ceramid with } 3 \text { PEG } \\
\text { units }\end{array}$ \\
\hline $\mathrm{Gb}_{3} \mathrm{PEG}_{3} \mathrm{C}_{24: 1} \mathrm{OH}$ & $\begin{array}{l}\text { BODIPY labeled 2-R-hydroxyl-nervonoyl globotriaosyl ce- } \\
\text { ramid with } 3 \text { PEG units }\end{array}$ \\
\hline $\mathrm{GM}_{1}$ & pentasaccharide ganglioside \\
\hline GNP & gold nano particle \\
\hline GPMV & giant plasma-membrane vesicle \\
\hline GUV & giant unilamellar vesicle \\
\hline HeLa cells & cervical cancer cells from Henrietta Lacks 2 \\
\hline HUS & haemolytic uraemic syndrome \\
\hline iSCAT & interferometric scattering microscopy \\
\hline ITO-slide & Indium Tin Oxide coated slide \\
\hline$K_{\mathrm{d}}$ & dissociation constant \\
\hline laser & light amplification by stimulated emission of radiation \\
\hline$l_{\beta}$ & gel like \\
\hline$l_{\mathrm{d}}$ & liquid disordered \\
\hline LED & light-emitting diode \\
\hline L-scan FCS & line-scan fluorescence correlation spectroscopy \\
\hline LMM & linear mixed-effects model \\
\hline$l_{\mathrm{o}}$ & liquid ordered \\
\hline$\% l_{\mathrm{o}}$ & liquid ordered phase distribution \\
\hline LUV & large unilamellar vesicle \\
\hline MD & molecular dynamics simulation \\
\hline 12MDD & 12-mercapto-1-dodecanol \\
\hline $2 \mathrm{ME}$ & 2-mercapto-1-ethanol \\
\hline $2 \mathrm{ME}-\mathrm{Au}$ & 2-mercapto-1-ethanol on gold \\
\hline $6 \mathrm{MH}$ & 6-mercapto-1-hexanol \\
\hline $6 \mathrm{MH}-\mathrm{Au}$ & 6-mercapto-1-hexanol on gold \\
\hline 16MHD & 16-mercapto-1-hexadecanol \\
\hline 16MHD-Au & 16-mercapto-1-hexadecanol on gold \\
\hline MLV & multi-lamellar vesicle \\
\hline $8 \mathrm{MO}$ & 8-mercapto-1-octanol \\
\hline 8MO-Au & 8-mercapto-1-octanol on gold \\
\hline 11MUD & 11-mercapto-1-undecanol \\
\hline 11MUD-Au & 11-mercapto-1-undecanol on gold \\
\hline$N$ & numbers of measurements \\
\hline naphthopyrene & naphtho[2,3-a]pyrene \\
\hline
\end{tabular}


NBD

OT

OT-Au

PBS

PC

PE

PEG

pHBMEC

PIP

POPC

POPE

POPS

PS

PSF

p- $-\mathrm{Si}_{3} \mathrm{~N}_{4}$

PSM

QI

Rhod-DOPE

SAM

SEM

$\mathrm{Si}_{3} \mathrm{~N}_{4}$

$\sigma$

$\mathrm{SiO}$

$\mathrm{SiO}_{2}$

$\mathrm{SiO}_{x}$

SM

$\mathrm{SM} \mathrm{C}_{16: 0}$

$\mathrm{SM} \mathrm{C}_{18: 0}$

$\mathrm{SM} \mathrm{C}_{20: 0}$

$\mathrm{SM} \mathrm{C}_{22: 0}$

$\mathrm{SM} \mathrm{C}_{24: 0}$

$\mathrm{SM} \mathrm{C}_{24: 1}$

$\mathrm{SM}_{\text {egg }}$

$\mathrm{SM}_{\text {porc }}$

SPR

s-PSM 7-nitrobenz-2-oxa-1,3-diazol-4-yl

octan-1-thiol

octan-1-thiol on gold

phosphate-buffered saline

phosphatidylcholine

phosphatidylethanolamine

polyethylene glycol

primary human blood microvascular endothelial cells

phosphatidylinositolphosphate

1-palmitoyl-2-oleoyl-sn-glycero-3-phosphatidylcholine

1-palmitoyl-2-oleoyl-sn-glycero-3-phosphatidylethanolamine

1-palmitoyl-2-oleoyl-sn-glycero-3-phosphatidy-L-serine

phosphatidylserine

point spread function

plasma cleaned silicon nitride

pore-spanning membrane

quantitative imaging

1,2-dioleoyl-sn-glycero-3-phosphoethanolamine-N-(lissamine rhodamine B sulfonyl)

self-assembled monolayer

scanning electron microscopy

silicon nitride

membrane tension

silicon monooxide

silicon dioxide

silicon oxide

sphingomyelin

palmitoyl sphingomyelin

stearoyl sphingomyelin

arachidoyl sphingomyelin

behenoyl sphingomyelin

lignoceroyl sphingomyelin

nervonoyl sphingomyelin

egg sphingomyelin

porcine brain sphingomyelin

surface plasmon resonance

solid supported pore-spanning membrane 
SPT

SSM

STx

STxA

STxB

SUV

$T$

TexasRed-DHPE

$T_{\mathrm{M}}$

Triton X-100

UK

US

UV

vis

Z-scan FCS single particle tracking

solid supported membrane

shiga toxin

A-subunit of shiga toxin

B-subunit of shiga toxin

small unilamellar vesicle

temperature

sulforhodamine-1,2-dihexanoyl-sn-glycero-3-

phosphoethanolamine

transition temperature

polyethylene glycol p-(1,1,3,3-tetramethylbutyl)-phenyl ether

United Kingdom

United States

ultraviolett light

visible light

Z-scan fluorescence correlation spectroscopy 



\section{Table of contents}

Abstract i

Abbreviations iii

1. Introduction 1

1.1. Enterohemorrhagic Escherichia coli . . . . . . . . . . . . . . . . . . 1

$1.2 . \quad$ Shiga toxin . . . . . . . . . . . . . . . . . 2

1.3. Cell membranes and their properties . . . . . . . . . . . . . . . 5

\begin{tabular}{ll}
\hline 2. Scope of the thesis & 9
\end{tabular}

3. Materials and methods 11

3.1. Used materials . . . . . . . . . . . . . . . . . . . . . . . . . 11

3.1.1. Matrix lipids . . . . . . . . . . . . . . . . . . 11

3.1.2. Membrane fluorescent probes . . . . . . . . . . . . . 13

3.1.3. Globotriaosyl ceramide . . . . . . . . . . . . . . . . 16

3.1.4. B-subunit of shiga toxin . . . . . . . . . . . . . . . . 18

3.2. $\quad$ Preparative methods . . . . . . . . . . . . . . . . . . . . . . 19

3.2.1. Preparation of vesicles . . . . . . . . . . . . . . . . . . . . . 19

3.2.2. Functionalization of porous substrates . . . . . . . . . . 21

3.2.3. Preparation of pore-spanning membranes (PSMs) . . . . . . . 22

3.3. Biophysical methods . . . . . . . . . . . . . . . . 23

3.3.1. Fluorescence microscopy . . . . . . . . . . . . . . . . . . . . 23

3.3.2. Interferometric scattering microscopy . . . . . . . . . . . . . 26

3.3.3. Atomic force microscopy . . . . . . . . . . . . . . . . . . 27

3.3.4. Surface plasmon resonance . . . . . . . . . . . . . . 28

3.4. Data evaluation . . . . . . . . . . . . . . . . . . . . 30

3.4.1. Phase distribution in GUVS . . . . . . . . . . . . . . . . 30

3.4.2. Phase separation on PSMs . . . . . . . . . . . . . . . . . 32

4. Phase behavior of globotriaosyl ceramide $\left(\mathrm{Gb}_{3}\right) \quad 37$

4.1. $\quad$ Binding studies of STxB to its receptor lipid $\mathrm{Gb}_{3} \ldots \ldots$. . . . . . . 37 
4.2. Analysis of the phase preference of labeled $\mathrm{Gb}_{3} \mathrm{~S} \quad \ldots . . \ldots$

4.2.1. Fluorescent properties of labeled $\mathrm{Gb}_{3} \mathrm{~S}$. . . . . . . . . . . . . 41

4.2.2. $\quad$ Preference of fatty acid labeled $\mathrm{Gb}_{3} \mathrm{~s}$ in phase-separated GUVs 46

4.2.3. Preference of head group labeled $\mathrm{Gb}_{3} \mathrm{~s}$ in phase-separated GUVs 49

4.3. Conclusion of phase behavior of $\mathrm{Gb}_{3} \ldots \ldots \ldots$

5. Pore-spanning membranes (PSMs) 63

5.1. Different functionalization strategies and their effects on PSMs . . . 64

5.1.1. Visualization of PSMs . . . . . . . . . . . . . 65

5.1.2. Membrane tension modulation of PSMs . . . . . . . . . . . . 71

5.1.3. Diffusion in PSMs . . . . . . . . . . . . . . . . . . 76

$5.2 . \quad$ Phase separation in PSMs . . . . . . . . . . . . . . . . . . . 82

5.3. Conclusion of PSMs . . . . . . . . . . . . . . . . . . . . . . 91

\begin{tabular}{ll}
\hline 6. & Conclusion \\
\hline
\end{tabular}

\begin{tabular}{ll}
\hline 7. References & 95
\end{tabular}

\begin{tabular}{ll}
\hline A. Appendix & 123
\end{tabular}

A.1. List of figures . . . . . . . . . . . . . . . . . . . . 123

A.2. List of schemes . . . . . . . . . . . . . . . . . . . . . 124

A.3. List of tables . . . . . . . . . . . . . . . . . . . . . . . . . 125

A.4. Lipid diffusion constants . . . . . . . . . . . . . . . . . 126

A.5. Membrane tension of pore-spanning membranes . . . . . . . . . . . . 128

A.6. Fluorescence labeled $\mathrm{Gb}_{3}$. . . . . . . . . . . . . . . . . . . . . . 129

A.7. Statistical analyses . . . . . . . . . . . . . . . . . . . 130

A.7.1. Statistical analysis of fatty acid labeled $\mathrm{Gb}_{3}$. . . . . . . . . 130

A.7.2. Statistical analysis of head group labeled $\mathrm{Gb}_{3}$. . . . . . . . 131

A.7.3. Statistical analysis of Membrane tension . . . . . . . . . . 133

A.8. Chemicals and consumables . . . . . . . . . . . . . . . . . . . 135

A.9. Software . . . . . . . . . . . . . . . . . . . . . 137

A.10. Equipment . . . . . . . . . . . . . . . . . . 137 


\section{Introduction}

\subsection{Enterohemorrhagic Escherichia coli}

Enterohemorrhagic Escherichia coli (EHEC) is a bacterial strain, which triggers many outbreaks around the world, including industrialized nations. 3 6] This bacterial strain was found in 1977 and the toxin was called vero toxin, a toxin that destroyed the vero cells. 7 EHEC became prominent in the 1980s, namely with large scale outbreaks in the United States (US) from the EHEC-strain O157:H7 in 1982.8 In 1996, EHEC was prominent in Japan with 9451 infected people. 3 In 2006, there was again a large outbreak in the US which involved 26 states. 9 Not only in the US and Japan were EHEC outbreaks, but also in Europe, e.g. Sweden $2002[10$ and Norway 2006 111 such outbreaks appeared.

In Germany, there were two outbreaks. The first appeared in 1995 in Bavaria, 12 the second 2011 in northern Germany. 13 18 To recognize such outbreaks, a reporting requirement for EHEC disease was introduced 1998 in Germany. 19. In the first years (2001 to 2003) of the reporting system, around 1000 peoples in Germany yearly got infected by EHEC.19 In the last six years, more than 2000 people got infected by EHEC per year (figure 1.1). 20

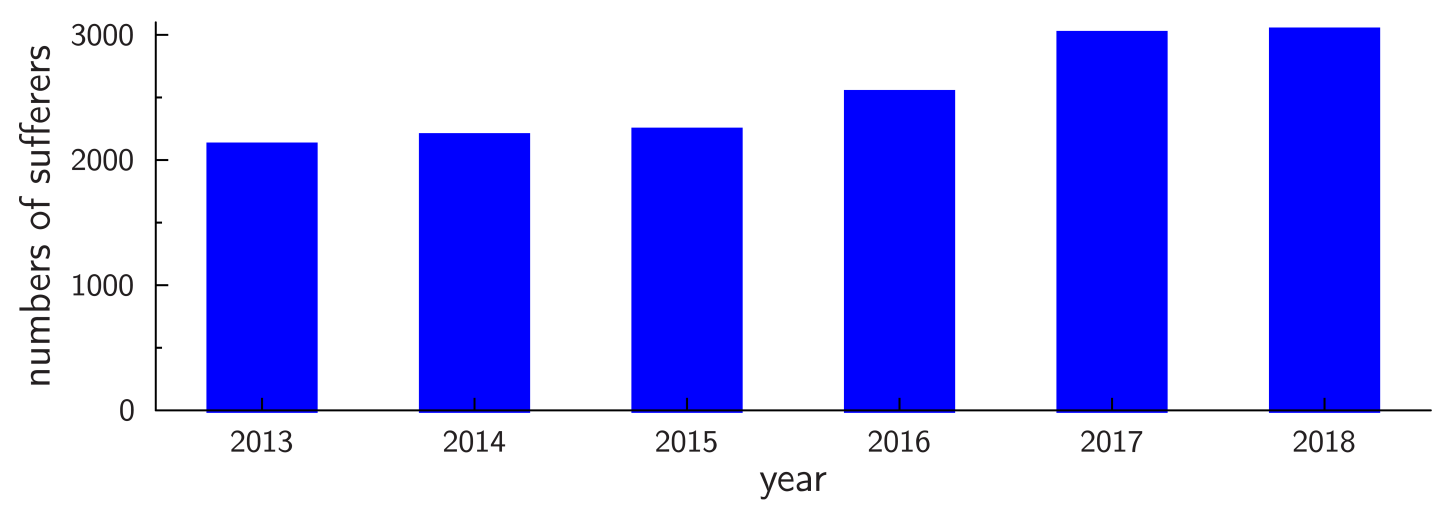

Figure 1.1.: The reported cases of enterohemorrhagic Escherichia coli (EHEC) disease in Germany from 2013 to 2018. The data was taken from the Robert Koch-Institut with SurvStat@RKI 2.0 at 30.07.2019.20] 
The effects of the disease caused by EHEC ranges from diarrhea, 21|22 bloody diarrhea, $23 \sqrt[25]{25}$ hemorrhagic colitis, 26,28 haemolytic uraemic syndrome (HUS) $1729 \sqrt{23}$ to the damages of the central nervous system. $30[34$ Some patients even died from EHEC infection due to multiple organ failure. 19

The different symptoms could be explained by different bacterial strains. 35 36. The different bacterial strains produce the same toxin, but different sub types due to a different binding affinity to the cells. The toxin of EHEC is the protein shiga toxin (STx) which is also the toxin from Shigella dysenteriae. 37

The uptake of the EHEC happens via food, e.g. raw meat 438 or unwashed vegetables. $\frac{39}{39}$ Within the intestine, the bacteria produces STx.40 The toxin activates the innate immunity. This results in a change of the cell morphology and in a change of the intercellular tight junctions in the intestinal epithelial. The STx can then cross the intestinal epithelial barrier into the blood stream. ${ }^{40}$ Through the blood stream, the STx is distributed into the whole body. The cell membrane of different tissues in the human body has different amounts of globotriaosyl ceramide $\left(\mathrm{Gb}_{3}\right)$ which is the receptor lipid of STx in the outer membrane leaflet.4143 The microvascular endothelial cells of the glomerular and brain have a high amount of the receptor lipid $\mathrm{Gb}_{3}$ in the outer membrane leaflet.44 47 This correlates with the disease caused by EHEC, HUS damage of the kidney (glomerular cells) and damage of the central nervous system. A topographical analysis of the distribution from $\mathrm{Gb}_{3}$ in a whole human body has not been done yet, but in a study with a whole weaned piglet. The topographical analysis showed the highest amount of $\mathrm{Gb}_{3}$ in the intestine and the lung. 48 The amounts of $\mathrm{Gb}_{3}$ in the tissues were ranked, the kidney cells are only at rank 8 and the cells of the brain are at rank 21 of altogether 25 ranks. 48 .

\subsection{Shiga toxin}

The toxin from EHEC is the STx which kills cells. To understand the mechanism of the toxicity from EHEC, a deeper look at the structure and uptake of STx is necessary. The STx is a $\mathrm{AB}_{5}$ toxin with one toxic A-subunit of STx (STxA) and five binding subunits the B-subunits of STx (STxB) (figure $1.2 \mathrm{~A}$ ). 4952 The five $\mathrm{STxB}$ form a pentameric ring (figure $1.2 \mathrm{~B}$ ). The STxA binds with a $\alpha$-helix into the middle of the ring (figure $1.2 \mathrm{~A}$ ). 51 The receptor lipid $\mathrm{Gb}_{3}$ has 15 binding sides in the pentameric structure of STxB (figure 1.2 C). 50

Cells which were incubated with STx and had $\mathrm{Gb}_{3}$ at the outer leaflet died, because the STxA binds to the ribosomes and blocks the protein biosynthesis. 53 The STx cannot diffuse through the membrane. The mechanism of the up take of STx into the 

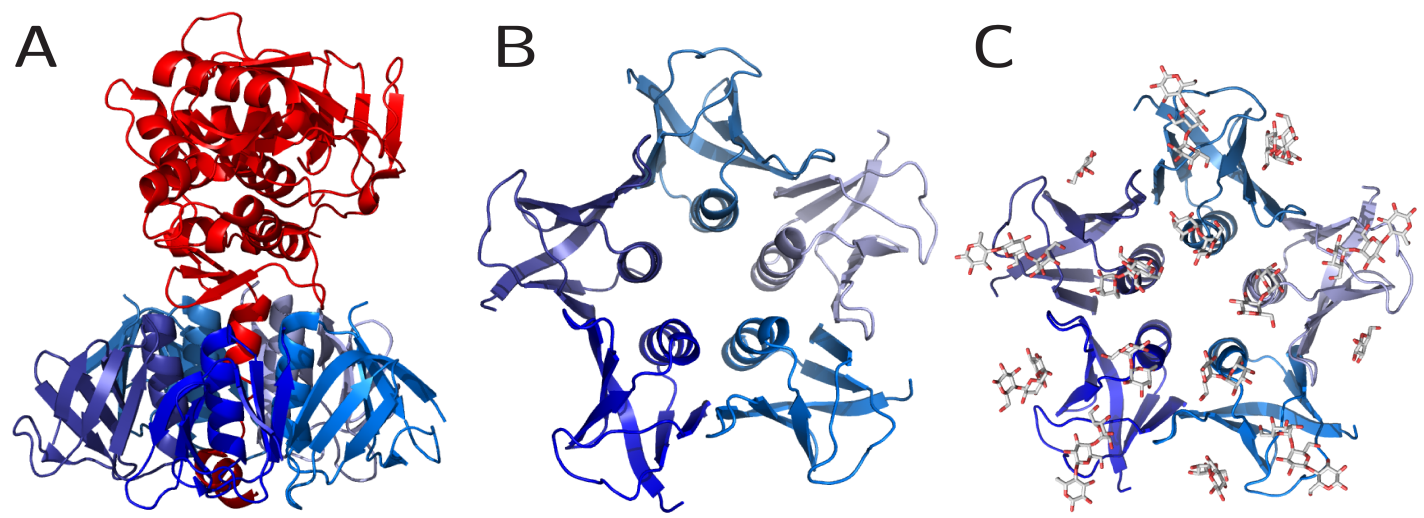

Figure 1.2.: The full protein shiga toxin (STx) is shown in A. The dark blue subunit is an A-subunit of shiga toxin (STxA) and the pentamer structure underneath are five B-subunit of shiga toxin (STxB) (PDB ID: 4M1U). 51 The pentamer structure of STxB is separately shown from the membrane site in B (PDB ID: 1C48). The 15 globotriaosyl ceramide $\left(\mathrm{Gb}_{3}\right)$ bindings sites of the pentameter of STxB are visualized in C (PDB ID: 1BOS).50

cell is shown in (figure 1.3). The initiate step of the uptake of STx into the cell is the binding of $\mathrm{STxB}$ to its receptor lipid $\mathrm{Gb}_{3}$ at the outer leaflet of the plasma membrane which is a heterogeneous two-dimensional fluid of proteins and lipids and is explained in the next chapter 1.3. The STx clusters on the membrane, 54 builds up membrane invaginations 55 and endocytotic pits. 56 The 5 The endocytotic pits are absorbed by the cells through clathrin-dependent $58 \mid 59]$ or clathin-independent endocytosis. 60[61 In the early endosomes, the STx are directed towards the late endosomal traffic route. The STx path to the endoplasmic reticulum goes from the late endosomes through the trans-Golgi network into the endoplasmic reticulum. In the endoplasmic reticulum, the membrane-associated endoprotease furin cuts the STx between the amino acids Arg251-Met252 from the STxA and activates its toxicity. 62 In this step, the translocation of STxA from the inside of the endoplasmatic reticulum into the cytosol takes place as well. 63 In the cytosol, the activated STxA inhibits the protein biosynthesis at the rRNA-N-glykosidase which kills the cells. $53[64$

An interesting fact is that cancer cells also have a high amount of $\mathrm{Gb}_{3}$ at the outer membrane leaflet 65 and the usage of STx for the cancer therapy is under research. 68 70] The nice STx uptake mechanism of a cell is perfect for transporting therapeutic proteins into the ensoplasmic reticulum or directly into the cytosol.69/71] There are different approaches to design anti-cancer drugs with STxB. A linker residue combined with a drug is chemically synthesized to the STxB pentamer.72 74 The design of fusion proteins was also evaluated. For that purpose, the toxic part of STxA was substituted by a therapeutic protein.75. Also, multi-lamellar vesicles (MLVs) coated with STxB at the outer leaflet were used, with anti cancer drugs on the 
क

8

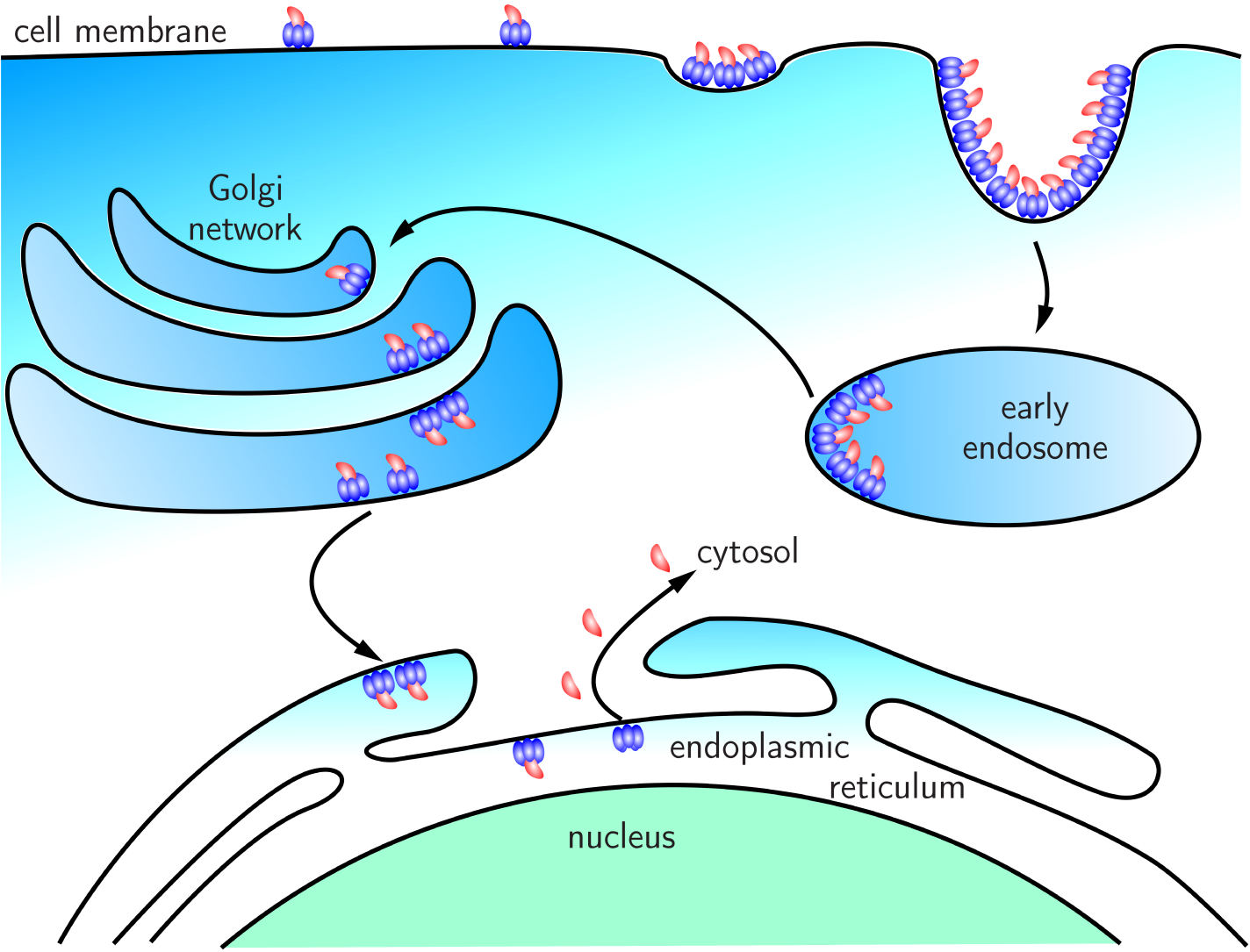

Figure 1.3.: Shiga toxin (STx) is taken up from the cell via binding to the cell membrane and clusters. The clusters build endocytotic pits and are taken up into the early endosomes. The STx is directed by the early entosomes to the retrograde traffic route over the Golgi network into the endoplasmatic reticulum. There, the STxA is cut from the STx and is translocated into the cytosol, where it inhibits the ribosomes. $62[64$

inside. 76 In all those cases, the drugs for the cancer cells are taken up from all cells with $\mathrm{Gb}_{3}$ on the outer leaflet. In the cancer therapy, the STxB can be mutated to have a better selection on the cell type and $\mathrm{Gb}_{3}$ concentrations.

The better understanding of the binding from STx to the cell membrane would help to design better drugs against EHEC and to design a selective drug against cancer. One important aspect is to know where the $\mathrm{Gb}_{3} \mathrm{~s}$ are concentrated before STx binds at the cell membrane. 


\subsection{Cell membranes and their properties}

The cell membrane is not only a homogeneous two dimensional fluid, which Singer and Nilcolson suggested. 77 The plasma membrane of an eucaryotic cell has many different components which can be divided into two major groups, lipids and membrane proteins. The lipid builds up the membrane matrix in which the membrane proteins are incorporated. The membrane has many different lipids which are divided into the subgroups glycerolipids, sterols and sphingolipids. $28 / 79$ The glycerolipids as well as the sphingolipids have different head groups. Very often, a phosphate is present in the lipid head group called phopholipids, which can be either a glycerolipid or a sphingolipid. The main lipid content are the phosphoglycerolipids, which are divided into the different head groups, phosphatidylcholine (PC), phosphatidylethanolamine (PE), phosphatidylinositolphosphate (PIP) and phosphatidylserine (PS). The glycerolipids as well as the sphingolipids have different fatty acids.

The membrane has a huge amount of different lipids aggregated into a two dimensional fluid. The different lipids and different proteins cluster in different ways and amounts which results in a heterogeneous membrane. 80 .83 These clusters or domains are called lipid rafts (figure 1.4 . 80 Lipid rafts are defined as "small (10-200 $\mathrm{nm}$ ), heterogeneous, highly dynamic, sterol- and sphingolipid-enriched domains that compartmentalize cellular processes. Small rafts can sometimes be stabilized to form larger platforms through protein-protein and protein-lipid interactions." 84 Three different lipid rafts are illustrated in figure 1.4. First, different proteins are activated in the lipid raft which are inactive in the non-raft membrane (figure 1.4 ). $.85[86$ It was reported that the actin network binds to a lipid raft. 87 The cell used the glycosphingolipids for signaling processes and communications which are enriched in such lipid rafts. 88 The last illustrated lipid raft contains the receptor lipid for STx it is $\mathrm{Gb}_{3}$ and the STx binds to it. 64] It is not clear if the lipid rafts exist first, before the toxin bind, or if the lipid raft is created by the binding of the toxin. A problem is that such lipid rafts are not visualized with the newest technical equipment in eukaryotes. 89 Only indirect information from detergent restistant membrane (DRM), ${ }^{90}$ fluorescence resonance energy transfer (FRET) ${ }^{91 \mid 92}$ and fluorescence correlation spectroscopy (FCS) ${ }^{93[94]}$ measurements hints that such rafts exist. In bacterial small domains of $\sim 40 \mathrm{~nm}$ in diameter were found. 95

The heterogeneity of the lipid raft model was analyzed in model membrane systems with the three major membrane lipids of eucaryotic cells, a low melting phospholipid (POPC or DOPC), a high melting lipid (DPPC or sphingomyelin (SM)) and sterol (cholesterol $(\mathrm{Chol})$ ). 9798 These model membranes undergo phase separation in a wide 


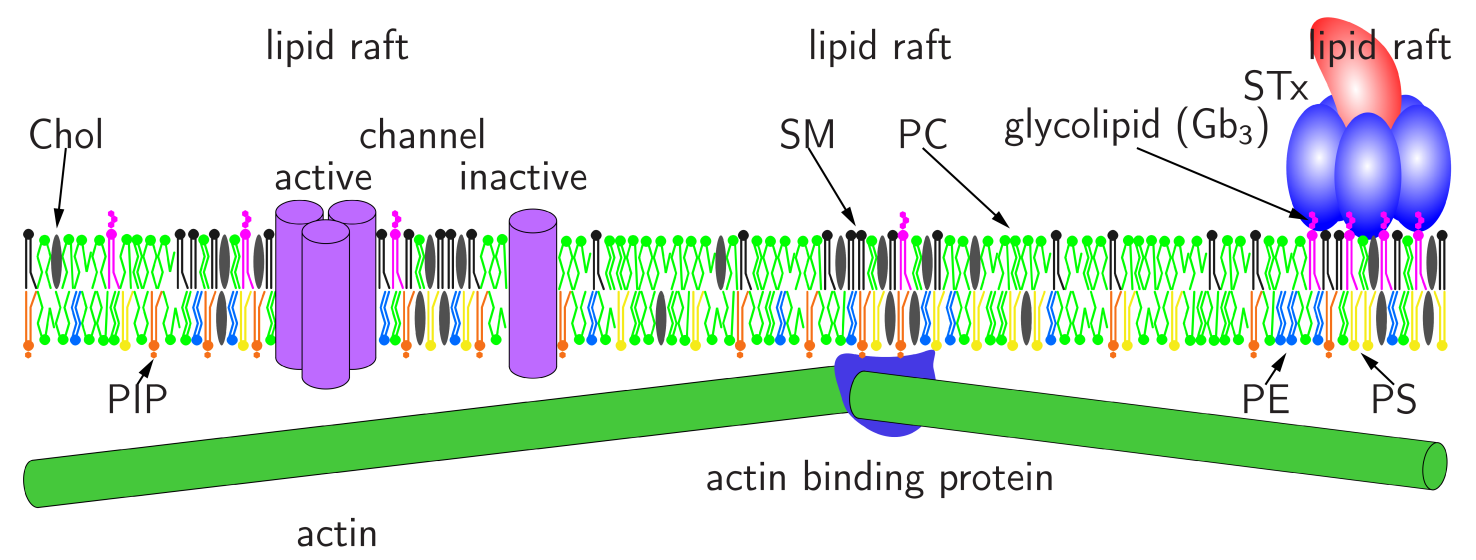

Figure 1.4.: A schematic drawing of the cell membrane with three different lipid rafts. From left to right, the first lipid raft activates a channel protein, 85 with the second one the actin is bound 87 and in the third one glycolipids are enriched and shiga toxin (STx) binds to it. 88 The cell membrane has different lipid species such as cholesterol (Chol), sphingomyelin (SM), phosphatidylcholine (PC), glycolipid as globotriaosyl ceramide $\left(\mathrm{Gb}_{3}\right)$, phosphatidylinositolphosphate (PIP), phosphatidylethanolamine (PE) and phosphatidylserine (PS). 96

region of the lipid compositions. 99 The lipid raft hypothesis is mimicked by such phase-separated membranes. The two phases of the raft mimic model membranes are the liquid ordered $\left(l_{\mathrm{o}}\right)$ phase which is enriched in high melting lipids and sterols and mimics the raft domains and the liquid disordered $\left(l_{\mathrm{d}}\right)$ phase which is enriched in low melting lipids and mimics the membrane matrix. The non-physiological lipid DOPC is preferential used instead of POPC, because the domains are larger and better visible with the fluorescent microscope. $\frac{97}{}$ The raft mimic model membranes which represent the cell membranes and the phase distribution of the STx receptor lipid will be analyzed. These data are an indication of the position from $\mathrm{Gb}_{3}$ in the plasma membrane relative to the lipid raft theory.

The measurement of the raft like lipid composition can be accomplished on different membrane systems which can be the giant unilamellar vesicles (GUVs), 100 adhered GUVs, 101$]$ pore-spanning membranes (PSMs) 102 and solid supported membranes (SSMs) 103 (figure 1.5). GUVs and SSMs mimic the cell membrane with the same conditions for each membrane region (figure 1.5 A, D). The two different leaflets can have different surroundings. In GUVs the buffer inside can be different from the buffer outside of a GUV. 104|105] The different leaflets of the SSMs are distinguishable: One is in contact with the surface of the support and the other leaflet is in contact with the solution. SSMs are very easy to handle and the membrane is, in contrast to the GUVs, two dimensional which is useful for microscopy studies (figure 1.5 D). With the supported membrane, the SSMs mimic the membrane cell adhesion to a 
surfaces. The increase of water between support and the membrane of SSM makes sure that the surface interaction has no influence of the properties from integral membrane proteins. 106 Therefore polymer-supported membranes were developed which increase the water film between the membrane and the substrates from few nm to many nm. 107 This increase of the water layer also increases the activity of proteins in the SSMs and brings it nearer to the membrane system of GUVs which mimic the membrane part of a cell which has no contact. To combine the adhesion membrane model (SSM) and the membrane model without any adhesion (GUV), the whole cellular membrane is described, because the cellular membrane has parts with adhesion and parts without. The cell membrane interacts not only with the surface outside of the cell but it has also adhesion properties to the inside of the cell. 108 The actin network stabilizes the plasma membrane and interacts strongly over actin binding proteins with the membrane. $\frac{109}{10}$ This can be mimicked with membrane systems which have different adhesion properties such as the adhered GUVs and the PSMs (figure $1.5 \mathrm{~B}$ and C). Both membrane systems have an adhered area as in SSMs and in both membrane systems there are lipid areas where the membrane is as free-standing as in GUVs. $102 \mid 110$ These membrane systems mimic the cell membranes with different adhesion properties to a surface or to the actin network. 102

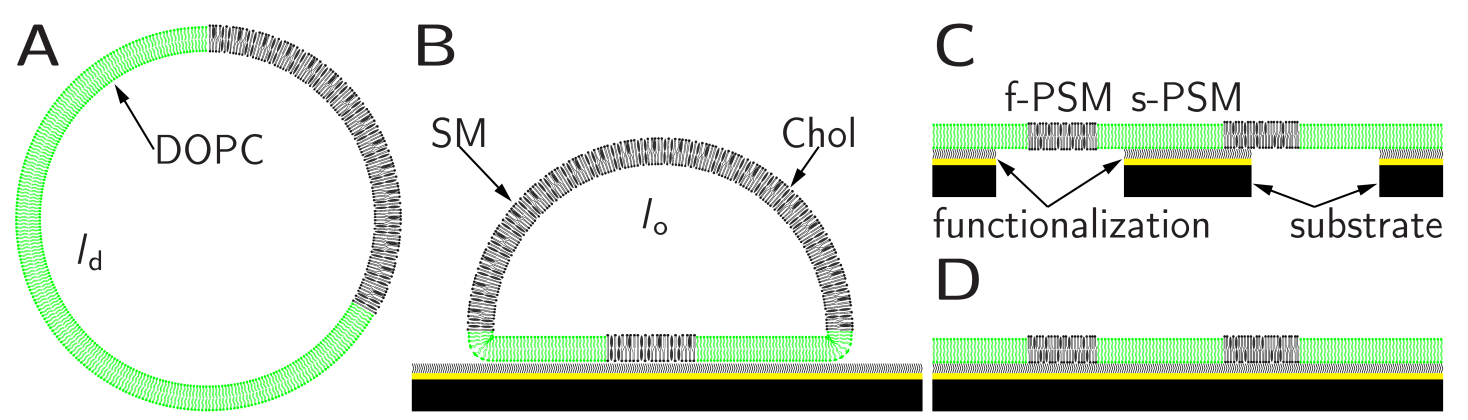

Figure 1.5.: Different model membrane systems are giant unilamellar vesicles (GUVs) (A), adhered GUVs (B), pore-spanning membranes (PSMs) (C) and solid supported membranes (SSMs). The shown systems are schematically drawn with phase-separated membranes, which contain DOPC/sphingomyelin $(\mathrm{SM}) /$ cholesterol $(\mathrm{Chol})$. The liquid disordered $\left(l_{\mathrm{d}}\right)$ phase is enriched in DOPC and the liquid ordered $\left(l_{\mathrm{o}}\right)$ phase is enriched in SM and Chol. The substrates for the adhered GUVs, PSMs and SSMs are functionalized with two different layers. The functionalization can differ for each system.

The actin network has an influence on the phase separation in membranes. Liu et al. showed that the $l_{\text {o }}$ phase is co-localized with the actin network. 87 This can be a rearrangement of lipids or that the phase diagram of such a lipid mixture changes with different adhesion regions which are adhesion to the action network or not. A phase diagram of PSMs was investigated which mimics such two different adhesion 
regions for one membrane. This model system should clarify if cell adhesion also has an influence on the raft building or if only the different lipid and protein components do that. These influences have then also an effect on the distribution of $\mathrm{Gb}_{3}$ in the plasma membrane. The distribution of the $\mathrm{Gb}_{3}$ was investigated with a raft mimic membrane system in which fluorescently labeled $\mathrm{Gb}_{3} \mathrm{~s}$ were incorporated. This analysis was done in GUVs. The knowledge of the distribution of the $\mathrm{Gb}_{3}$ in plasma membrane or plasma mimic membrane can help to understand the binding properties of $\mathrm{STxB}$ to $\mathrm{Gb}_{3}$ so that different drugs against EHEC can be designed which changes the $\mathrm{Gb}_{3}$ distribution in the plasma membrane and prevent the uptake of STx into the cell. 


\section{Scope of the thesis}

The bacterium enterohemorrhagic Escherichia coli (EHEC) is one of the main causes of food-borne illness around the world. The disease caused by EHEC ranges from different diarrheas over hemorrhagic colitis and haemolytic uraemic syndrome up to damage of the central nervous system, in some cases with lethal outcome. All these different diseases are only triggered by the toxic protein shiga toxin (STx) which inhibits the protein biosynthesis of eukaryotic cells. In a first step, while approaching the cells, the B-subunit of STx (STxB) binds to the receptor lipid globotriaosyl ceramide $\left(\mathrm{Gb}_{3}\right)$ which is present in the outer membrane of human cells. The cellular membrane is highly heterogeneous and has regions of various ordering. This might lead to enrichment or depletion of $\mathrm{Gb}_{3}$ in certain areas.

One important finding is that the $\mathrm{Gb}_{3}$, the receptor for STx, is enriched in membranes of cancer cell. A better understanding of the binding from STx to the cell membrane would help to improve drug development against EHEC and open pathways for drugs to selectively target cancer cells. One important point is the knowledge where $\mathrm{Gb}_{3} \mathrm{~s}$ are concentrated before STx binds to the cell membrane. Quantification of local $\mathrm{Gb}_{3}$ concentration in areas of different lipid order of biomimetic membranes is one scope within this thesis. For the quantification giant unilamellar vesicles (GUVs) and pore-spanning membranes (PSMs) are used which allow preparation of membranes with high definition to identify the impact of membrane ordering to $\mathrm{Gb}_{3}$ enrichment.

The regions of various ordering in the cellular membrane can be affected by heterogeneous connections to either the cytoskeleton in the inside of the cell or to different surfaces at the outside of the cell. A second scope of this thesis is to identify the effects related to membrane-surface interactions (adhesion) to the organization of membranes with regions of higher and lower ordering. This might also influence the preferential distribution of $\mathrm{Gb}_{3}$ in lipid membranes. 



\section{Materials and methods}

\subsection{Used materials}

\subsubsection{Matrix lipids}

In this work, different lipids with different functions were used. The lipid composition of the analyzed membranes with an amount of at least of $94 \%$ are summarized as matrix lipids and represent the three main compositions of mammalian lipids, glycerolipids, sphingolipids and sterols.78

\section{1,2-dioleoyl-sn-glycero-3-phosphatidylcholine (DOPC)}<smiles>CCCCCCCC/C=C\CCCCCCCCC(=O)OC[C@H](COP(=O)(O)OCC[N+](C)(C)C)OC(=O)CCCCCCCCCCCCC</smiles>

Scheme 3.1.: The structure of 1,2-dioleoyl-sn-glycero-3-phosphatidylcholine (DOPC).

1,2-dioleoyl-sn-glycero-3-phosphatidylcholine (DOPC) is a non-natural glycerophospholipid with two oleic acids in the hydrophobic part (Scheme 3.1). This phospholipid is used in many model systems and has a low transition temperature $\left(T_{\mathrm{M}}\right)$. The reported $T_{\mathrm{MS}}$ are $\left.-14{ }^{\circ} \mathrm{C}, 111\right]-16.5^{\circ} \mathrm{C}, \underline{112}-17.3^{\circ} \mathrm{C}, \underline{113}-18.3 \pm 3.6{ }^{\circ} \mathrm{C}, 114,-18^{\circ} \mathrm{C}, 115$ $-19.1^{\circ} \mathrm{C}, 116-21^{\circ} \mathrm{C} 117$ and $-22^{\circ} \mathrm{C}$. 118 The molecular weight of DOPC is $786.129 \mathrm{~g} \mathrm{~mol}^{-1}$ and the chemical formula is $\mathrm{C}_{44} \mathrm{H}_{84} \mathrm{NO}_{8} \mathrm{P}$.

\section{Cholesterol (Chol)}

Cholesterol (Chol) is a sterol (scheme 3.2). Chol has a molecular weight of $386.35 \mathrm{~g} \mathrm{~mol}^{-1}$ and its chemical formula is $\mathrm{C}_{27} \mathrm{H}_{46} \mathrm{O}$. It is present in mammalian membranes and reduces the fluidity in the liquid-crystalline phase. 119 Chol increases the fluidity for gel phase membranes. Sphingomyelin (SM) and Chol are the main lipid parts in the lipid rafts. $\frac{84}{3}$ 


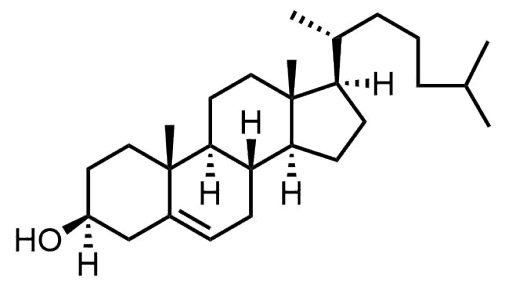

Scheme 3.2.: The structure of cholesterol (Chol).

\section{Sphingomyelin (SM)}

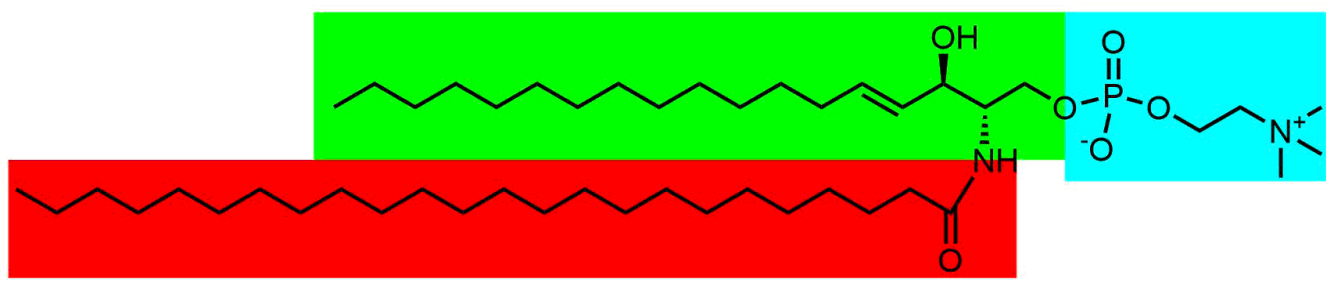

Scheme 3.3.: The structure of lignoceroyl sphingomyelin $\left(\mathrm{SM} \mathrm{C}_{24: 0}\right)$ is shown as an example of sphingomyelin (SM) structure. The sphingosine structure is labeled in green. The hydrophilic cholin-phosphate-ester group (blue) is connected with a phosphate-esters to the sphingosine. The lignoceric acid (red) is connected via an amide bond to the 2 amine group of the sphingosine.

Sphingomyelin (SM) has a sphingosine basic structure (figure 3.3 green). A cholin group (figure 3.3 blue) is connected at the 1 hydroxyl-group of the spingosine by a phosphate-ester. The fatty acid (figure 3.3, red) which is connected through a amide bond to the 2 amine group of the sphingosine differs in each SM species. The shown $\mathrm{SM} \mathrm{C}_{24: 0}$ has a lignoceric acid as a fatty acid. Porcine brain $\mathrm{SM}\left(\mathrm{SM}_{\text {porc }}\right)$ is a natural SMs extraction and was used in this work. $\mathrm{SM}_{\text {porc }}$ is a $\mathrm{SM}$ mixture with six known different SM species (table 3.1.

Table 3.1.: The natural sphingomyelin (SM) extraction, porcine brain sphingomyelin $\left(\mathrm{SM}_{\text {porc }}\right)$, has different amounts of different $\mathrm{SMs}$, which are listed here. The different extracted $\mathrm{SMs}$ are palmitoyl sphingomyelin $\left(\mathrm{SMC}_{16: 0}\right)$, stearoyl sphingomyelin ( $\mathrm{SM} \mathrm{C}_{18: 0}$ ), arachidoyl sphingomyelin ( $\left.\mathrm{SM} \mathrm{C}_{20: 0}\right)$, behenoyl sphingomyelin $\left(\mathrm{SM} \mathrm{C}_{22: 0}\right)$, lignoceroyl sphingomyelin $\left(\mathrm{SM} \mathrm{C}_{24: 0}\right)$ and nervonoyl sphingomyelin $\left(\mathrm{SM}_{24: 1}\right)$ and an unknown fraction. $120 \mid 121]$

\begin{tabular}{cllllllc}
$\mathrm{SMs}_{\mathrm{S}}$ & $\mathrm{SM} \mathrm{C}_{16: 0}$ & $\mathrm{SM} \mathrm{C}_{18: 0}$ & $\mathrm{SM} \mathrm{C}_{20: 0}$ & $\mathrm{SM} \mathrm{C}_{22: 0}$ & $\mathrm{SM} \mathrm{C}_{24: 0}$ & $\mathrm{SM} \mathrm{C}_{24: 1}$ & Unknown \\
\hline$\%$ & 2 & 49 & 5 & 8 & 6 & 20 & 10
\end{tabular}

The synthetic SMs which were used in this work are palmitoyl SM $\left(\mathrm{SMC}_{16: 0}\right)$, stearoyl SM ( $\left.\mathrm{SMC}_{18: 0}\right)$, arachidoyloyl $\mathrm{SM}\left(\mathrm{SMC}_{20: 0}\right)$, behenoyl $\mathrm{SM}\left(\mathrm{SMC}_{22: 0}\right)$ and lignoceroyl $\mathrm{SM}\left(\mathrm{SM} \mathrm{C}_{24: 0}\right)$. At room temperature, all $\mathrm{SMs}$ are in the gel-phase. The 
$T_{\mathrm{MS}}$ from the gel-phase to the liquid-crystalline phase for different SMs are listed in table 3.2. For some SMs, there are reported gel to gel-phase $T_{\mathrm{M}} \mathrm{s}$ which are also listed. SMs occur in mammalian cells and are contributed to the lipid raft theory. The lipid rafts are enriched in SMs and Chol. In model membranes, SMs are enriched in the liquid ordered $\left(l_{\mathrm{o}}\right)$ phase when the membrane undergoes phase separation into the liquid disordered $\left(l_{\mathrm{d}}\right)$ and $l_{\mathrm{o}}$ phase.

Table 3.2.: The different sphingomyelins (SMs) have different transition temperatures $\left(T_{\mathrm{MS}}\right)$. Some literature reported pre- $T_{\mathrm{MS}}$ which are also listed and separated with a comma. The various $T_{\mathrm{M}}$ from varying literature are separated with a semicolon. The different $\mathrm{SMs}$ are porcine brain sphingomyelin $\left(\mathrm{SM}_{\text {porc }}\right)$, palmitoyl sphingomyelin $\left(\mathrm{SM} \mathrm{C}_{16: 0}\right)$, stearoyl sphingomyelin ( $\mathrm{SM} \mathrm{C}_{18: 0}$ ), arachidoyl sphingomyelin ( $\mathrm{SMC}_{20: 0}$ ), behenoyl sphingomyelin $\left(\mathrm{SM} \mathrm{C}_{22: 0}\right)$, lignoceroyl sphingomyelin $\left(\mathrm{SM} \mathrm{C}_{24: 0}\right)$ and nervonoyl sphingomyelin $\left(\mathrm{SM} \mathrm{C}_{24: 1}\right)$.

\begin{tabular}{|c|c|}
\hline SMs & List of reported transition temperatures $\left[{ }^{\circ} \mathrm{C}\right]$ \\
\hline $\mathrm{SM}_{\text {porc }}$ & $35-40 ; 12035.7 \pm 5.8122$ \\
\hline $\mathrm{SM} \mathrm{C}_{16: 0}$ & 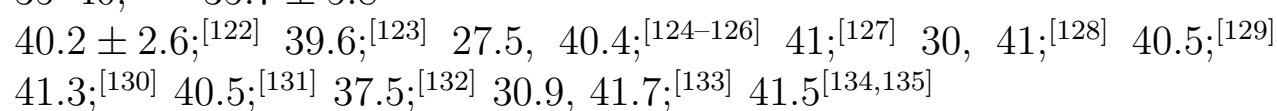 \\
\hline $\mathrm{SM} \mathrm{C}_{18: 0}$ & 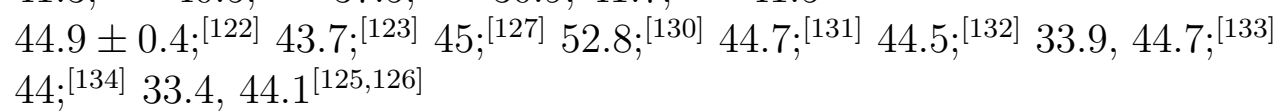 \\
\hline $\mathrm{SM} \mathrm{C}_{20: 0}$ & $46.5 ; 12212732.4,44.6125126$ \\
\hline $\mathrm{SM} \mathrm{C}_{22: 0}$ & $31.7,45.8 ; 124[126] 18.4,46.9 ; 127 \quad 44.5$\begin{tabular}{ll|l|l|l|l|}
122 & 132 \\
\end{tabular} \\
\hline $\mathrm{SM} \mathrm{C}_{24: 0}$ & 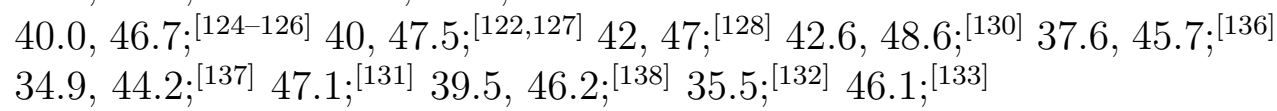 \\
\hline $\mathrm{SM} \mathrm{C}_{24: 1}$ & $18.0,22.3,27.4 ; 12314,16,23 ; 12826.2 ; 13127.5 ; 122132$ \\
\hline
\end{tabular}

\subsubsection{Membrane fluorescent probes}

To visualize the lipid bilayer under a microscope, different fluorescent probes were used. The used $l_{\mathrm{d}}$ phase marker are all coupled to DOPE or 1,2-dipalmitoyl-snglycero-3-phosphatidylethanolamine (DPPE). Different light amplifications by stimulated emission of radiation (lasers) were used to excite the fluorophores.

\section{ATT0488-DOPE}

ATTO488-1,2-dioleoyl-sn-glycero-3-phosphoethanolamin (ATTO488-DOPE) is a lipid coupled membrane fluorophore (scheme 3.4). The marked lipid is DOPE and the fluorophore is ATTO488. The excitation maximum is at the wavelength $500 \mathrm{~nm}$ and the emission maximum at the wavelength $520 \mathrm{~nm}$. The excitation with the laser line $488 \mathrm{~nm}$ is possible. This fluorophore is an $l_{\mathrm{d}}$ phase marker. In this work, the fluorophore was used to visualized membranes and phase-separated membranes. 


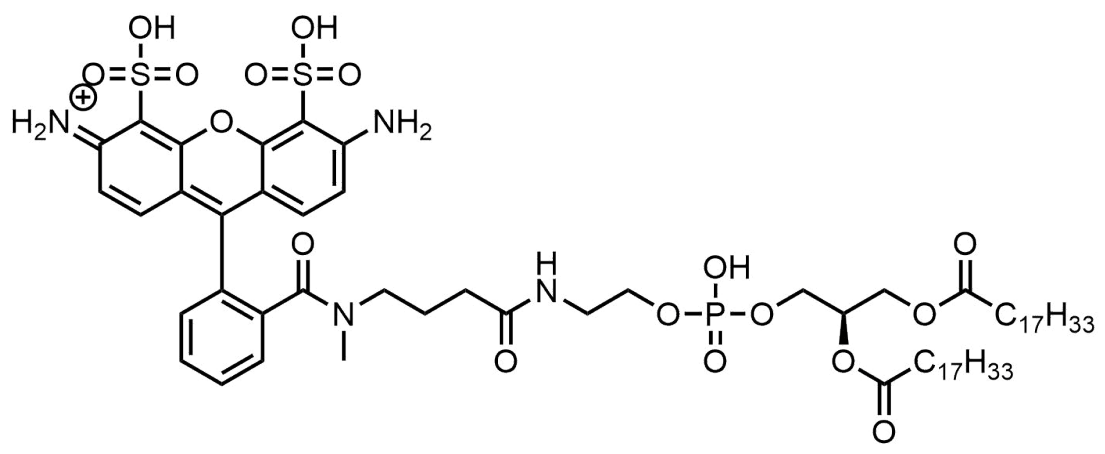

Scheme 3.4.: The structure of ATTO488-1,2-dioleoyl-sn-glycero-3-phosphoethanolamin (ATTO488-DOPE). The ATTO488 fluorophore is connected to a DOPE lipid.

\section{TexasRed-DHPE}

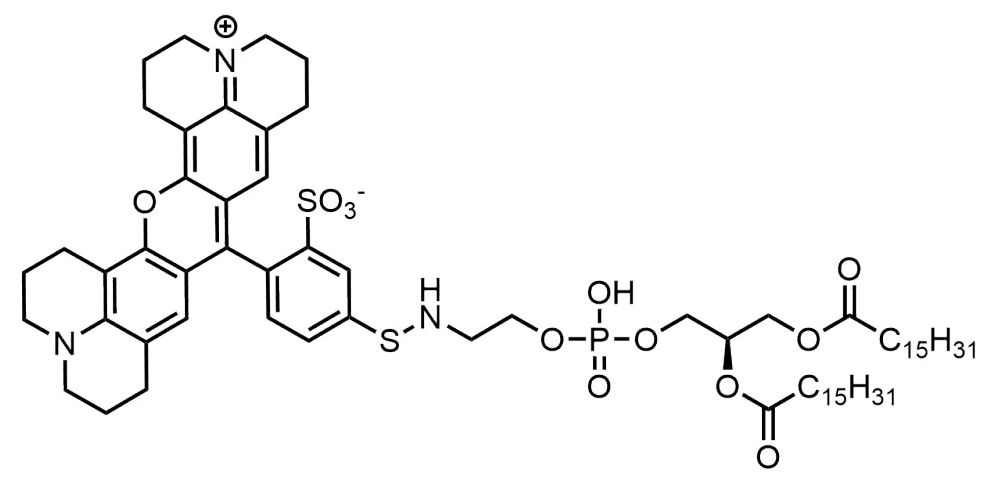

Scheme 3.5.: The structure of sulforhodamine-1,2-dihexanoyl-sn-glycero-3phosphoethanolamine (TexasRed-DHPE). The sulforhodamine fluorophore is connected to a DPPE lipid.

A $l_{\mathrm{d}}$ phase marker fluorophore is the sulforhodamine-1,2-dihexanoyl-sn-glycero3-phosphoethanolamine (TexasRed-DHPE).139|140 The sulforhodamine is connected to a DPPE lipid (scheme 3.5). This fluorophore was used to label membranes and phase-separated membranes. The diffusion constant of this fluorophore was determined with fluorescence correlation spectroscopy (FCS) in freestanding pore-spanning membranes (f-PSMs) composed of the DOPC membrane. The excitation maximum and emission maximum are $595 \mathrm{~nm}$ and $615 \mathrm{~nm}$. The fluorophore was excited with the laser wavelength $561 \mathrm{~nm}$.

\section{ATT0655-DOPE}

ATTO655-1,2-dioleoyl-sn-glycero-3-phosphoethanolamin (ATTO655-DOPE) is a lipid-coupled membrane fluorophore (scheme 3.6). The lipid is DOPE and the fluorophore is ATTO4655. The excitation maximum is at the wavelength $663 \mathrm{~nm}$ and 


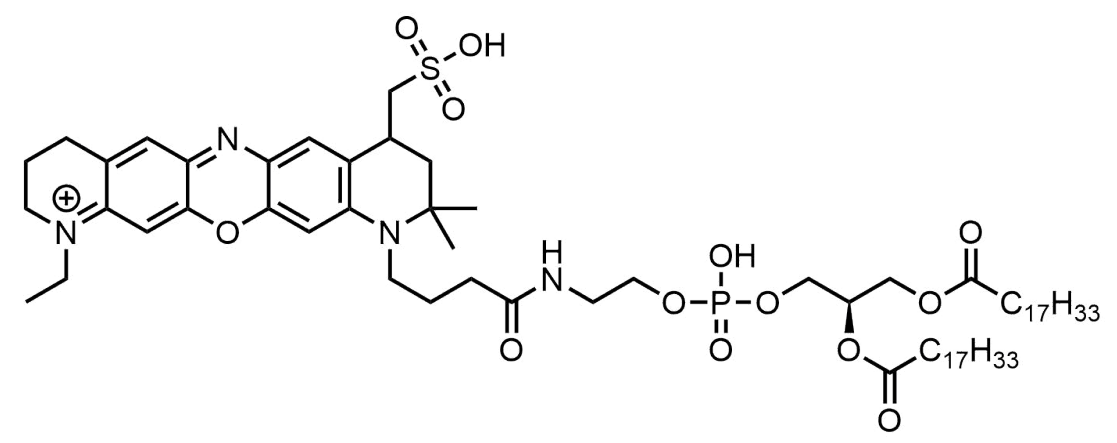

Scheme 3.6.: The structure of ATTO655-1,2-dioleoyl-sn-glycero-3-phosphoethanolamin (ATTO655-DOPE). The ATTO655 fluorophore is connected to a DOPE lipid.

the emission maximum is at the wavelength $680 \mathrm{~nm}$. The excitation with the laser line $633 \mathrm{~nm}$ is suitable and this fluorophore is an $l_{\mathrm{d}}$ phase marker. In this work the fluorophore was used to visualized membranes and to measure the diffusion constant with FCS in DOPC f-PSMs.

\section{Dy731-DOPE}

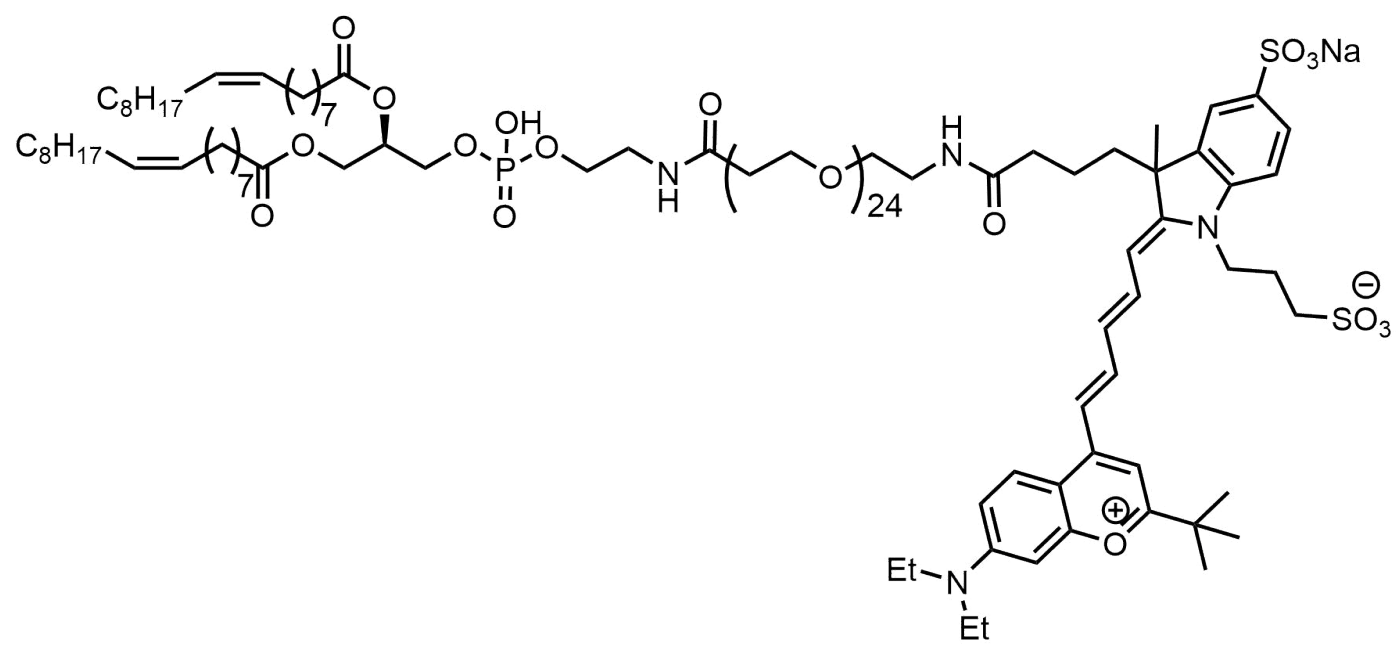

Scheme 3.7.: The structure of Dy731-PEG 25 -1,2-dioleoyl-sn-glycero-3-phosphoethanolamin (Dy731-DOPE). The Dy731 fluorophore is connected via 25 polyethylene glycol (PEG) units to a DOPE lipid.

Dy731-PEG ${ }_{25}$-1,2-dioleoyl-sn-glycero-3-phosphoethanolamin (Dy731-DOPE) is a lipid coupled fluorophore (scheme 3.7). The fluorophore (Dy731) was connected to a DOPE with 25 polyethylene glycol (PEG) units in between. This was done in the group of Prof. Dr. Daniel B. Werz. The excitation wavelength is $732 \mathrm{~nm}$ and the fluorophore emits by $763 \mathrm{~nm}$. The phase preference of this fluorophore is the $l_{\mathrm{d}}$ phase. 141 This fluorophore was used in the phase distribution measurements of 
globotriaosyl ceramide $\left(\mathrm{Gb}_{3}\right)$. It is possible to excite Dy731-DOPE with the laser wavelength $633 \mathrm{~nm}$.

\section{Naphthopyrene}

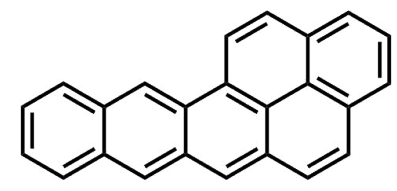

Scheme 3.8.: The structure of naphtho[2,3-a]pyrene (naphthopyrene).

The fluorophore naphtho[2,3-a]pyrene (naphthopyrene) has an excitation maximum at $459 \mathrm{~nm}$ and an emission maximum at $495 \mathrm{~nm}$. The structure of naphthopyrene is planar and has $24 \pi$ electrons (scheme 3.8). The phase preference of naphthopyrene is the $l_{\mathrm{o}}$ phase. $139 \mid 140$ In this work naphthopyrene was used to measure the diffusion constant with FCS in the $l_{\mathrm{o}}$ phase in the f-PSM. The excitation laser wavelength amounted to $458 \mathrm{~nm}$.

\section{BODIPY-Chol}

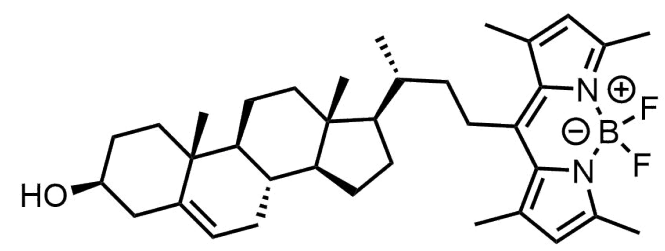

Scheme 3.9.: The structure of 23-(dipyrrometheneboron difluoride)-24-norcholesterol (BODIPY-Chol).

23-(dipyrrometheneboron difluoride)-24-norcholesterol (BODIPY-Chol) is a Chol derivative (scheme 3.9). The fluorophore BODIPY is connected to the 23'-C-atom of Chol. The BODIPY-Chol has the phase preference of Chol and is an $l_{\text {o }}$ phase marker.140|142] The emission maximum is at the wavelength $506 \mathrm{~nm}$ and the maximum of the excitation wavelength is at $499 \mathrm{~nm}$. BODIPY-Chol was stimulated with the laser line $488 \mathrm{~nm}$. The BODIPY-Chol was used to visualized the $l_{\mathrm{o}}$ phase in PSMs and the diffusion constant was measured in the $l_{\mathrm{o}}$ phase with FCS in f-PSMs.

\subsubsection{Globotriaosyl ceramide}

Globotriaosyl ceramide $\left(\mathrm{Gb}_{3}\right)$ is the receptor lipid of shiga toxin (STx). The receptor structure motive is the trisaccharide $\alpha$-D-galactose- $(1 \rightarrow 4)-\beta$-D-galactose- $(1 \rightarrow 4)$ - 


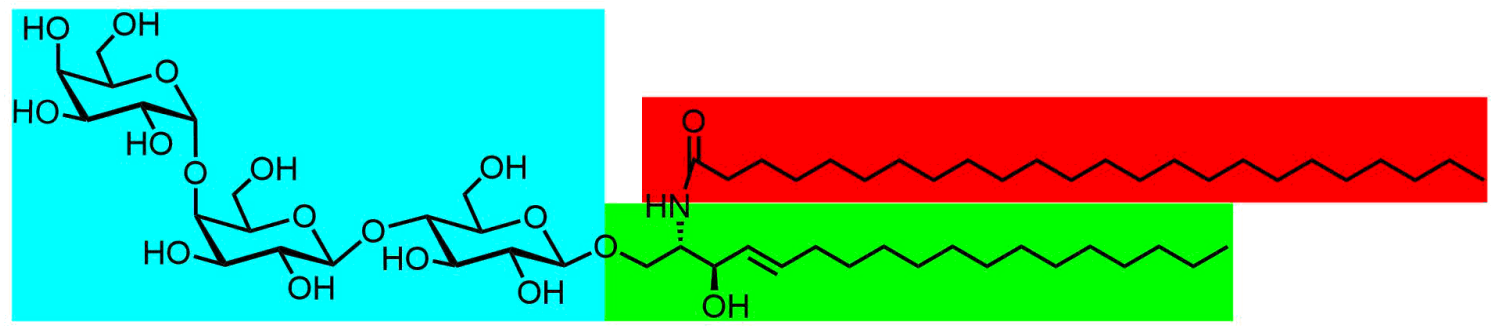

Scheme 3.10.: The structure of globotriaosyl ceramide $\left(\mathrm{Gb}_{3}\right)$ has a sphingosine motive (green). The head group of $\mathrm{Gb}_{3}$ consists of a trisaccheride which is $\alpha$-D-galactose- $(1 \rightarrow 4)-\beta$ D-galactose- $(1 \rightarrow 4)-\beta$-D-glucose (blue). The hydroxyl/aldehyde group from glucose 1 binds to the sphingosine 1 hydroxyl group via an acetal bond. The $\mathrm{Gb}_{3}$ can vary in the fatty acid (red) which is connected via an amide bond to the 2 amine group of the sphingosine. The shown $\mathrm{Gb}_{3}$ has a lignoceroyl fatty acid.

$\beta$-D-glucose (figure 3.10 blue). This is connected to a sphingosine skeleton with an acetal bond (figure 3.10 green). The fatty acid which is connected via an amide bond to the 2 amine of the sphingosine can vary in the $\mathrm{Gb}_{3}$ like the SMs (figure $3.10 \mathrm{red}$ ). A porcine erythrocytes $\mathrm{Gb}_{3}\left(\mathrm{~Gb}_{3 \text { porc }}\right)$ was used. The fatty acid mixture in the $\mathrm{Gb}_{3 \text { porc }}$ is composed of $21 \%$ palmitic, $4 \%$ stearic, $2 \%$ oleic, $14 \%$ behenic, $1 \%$ tricosylic, $19 \%$ lignoceric, $11 \%$ nervonic, $4 \% \alpha$-hydroxylated behenic, $5 \% \alpha$-hydroxylated lignoceric and $17 \% \alpha$-hydroxylated nervonic acids.

\section{Fatty acid labeled $\mathrm{Gb}_{3} \mathrm{~S}$}

Two different $\mathrm{Gb}_{3}$ s species with a labeled fatty acid were used in this work. The $\mathrm{Gb}_{3}$ phenyl-modified fatty acid $\left(\mathrm{Gb}_{3} \mathrm{PH}\right)$ and $\mathrm{Gb}_{3}$ thienothienyl-modified fatty acid $\left(\mathrm{Gb}_{3} \mathrm{TT}\right)$ were synthesized by the group of Prof. Dr. Daniel B. Werz.141] Both structures have an aromatic ring system, phenyl and thienothienyl respectively. The $\pi$ electron systems are expanded with conjugated double bonds. Five conjugated double-bonds in a trans configuration were synthesized at the phenyl ring to increase the wavelength of the absorption and emission. The carbon-chain between the phenyl group and the acid group has 25 carbon atoms. The thienothienyl $\pi$ electron system was expanded with three conjugated double-bonds in direction to the carbon acid and to the other direction with one double bond. All double bonds have the trans-configuration. There are 14 carbon atoms between the carbon acid and the thienothienyl ring system. The excitation wavelengths for the $\mathrm{Gb}_{3} \mathrm{PH}$ and $\mathrm{Gb}_{3} \mathrm{TT}$ are from 330 to $366 \mathrm{~nm}$ and 369 to $391 \mathrm{~nm}$ respectively. The emission maxima are at $434 \mathrm{~nm}$ and $440 \mathrm{~nm}$ respectively. 


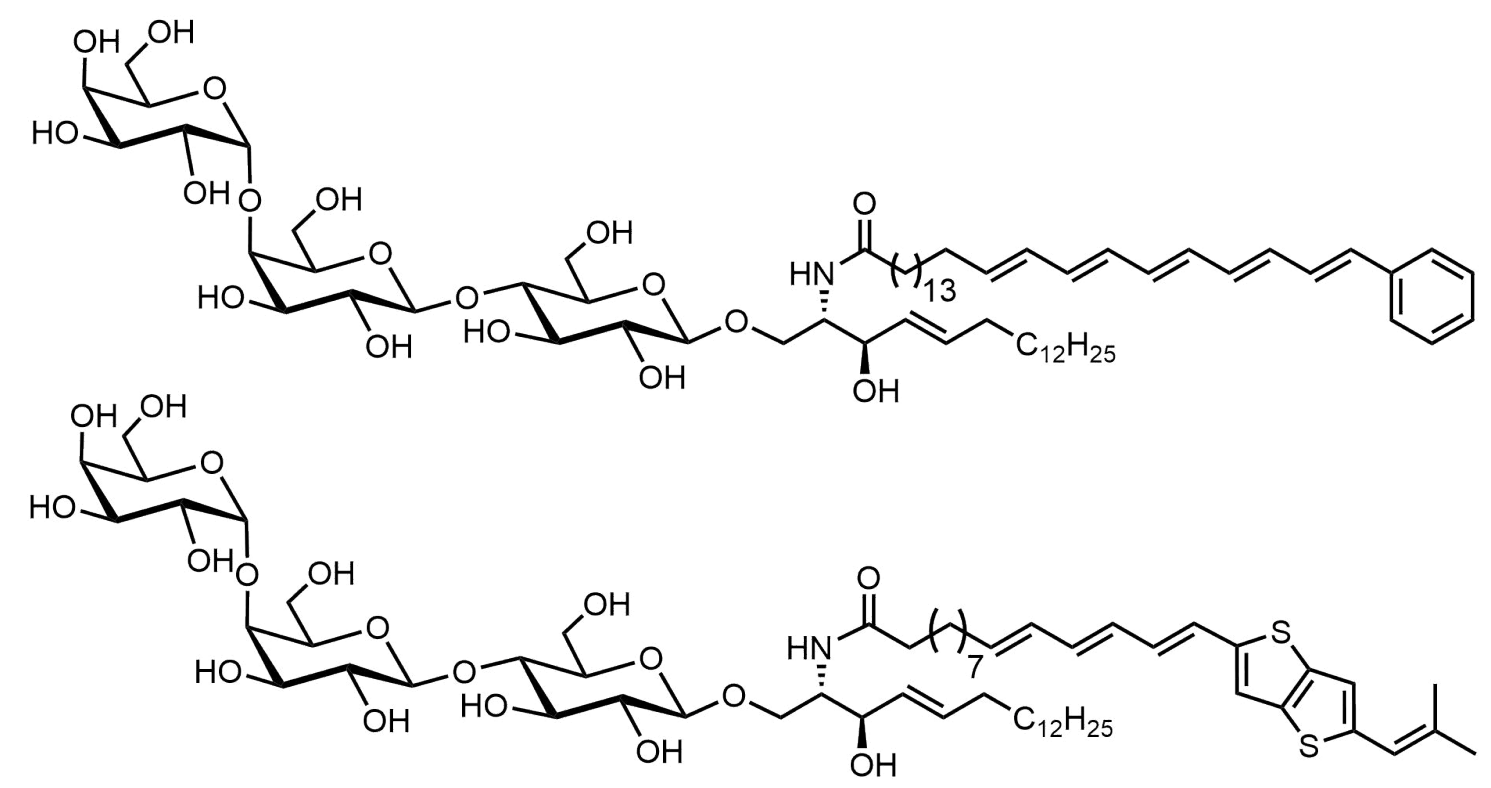

Scheme 3.11.: Structures of the two different fatty acid labeled globotriaosyl ceramides $\left(\mathrm{Gb}_{3} \mathrm{~s}\right)$. The structure of globotriaosyl ceramid phenyl-modified fatty acid $\left(\mathrm{Gb}_{3} \mathrm{PH}\right)$ is shown at the top, the structure of globotriaosyl ceramid thienothienyl-modified fatty acid $\left(\mathrm{Gb}_{3} \mathrm{TT}\right)$ below. Both fatty acids have a conjugated $\pi$ electron system in the ring structure and conjugated double bonds with them.

\section{head group labeled $\mathrm{Gb}_{3} \mathrm{~S}$}

The head group labeled $\mathrm{Gb}_{3} \mathrm{~s}$ were synthesized by the group of Prof. Dr. Daniel B. Werz (figure 3.12). 143 The group synthesized eight different head group labeled $\mathrm{Gb}_{3} \mathrm{~s}$. The fluorophore which is connected over a PEG linker to the 2'-hydroxylgroup of the $\beta$ galactose was in all cases BODIPY. The linker length (n) differs from 3 to 13 units. The carbon chain of the fatty acid ( $\mathrm{m}$ ) in each case has 24 carbon atoms, but it differs in the saturation $(\Delta)$ meaning the saturated fatty acid $(\Delta=0$, scheme 3.12 top structure) and mono-unsaturated fatty acid ( $\Delta=1$, scheme 3.12 bottom structure). The $\alpha$ position $(\mathrm{X})$ from the fatty acid is hydroxylated $(\mathrm{OH})$ or not $(\mathrm{H})$. In total, all head group labeled $\mathrm{Gb}_{3} \mathrm{~s}$ are abbreviated to $\mathrm{Gb}_{3} \mathrm{PEG}_{\mathrm{n}} \mathrm{C}_{\mathrm{m}: \Delta} \mathrm{X}$.

\subsubsection{B-subunit of shiga toxin}

The shiga toxin (STx) has six subunits, the A-subunit of STx (STxA) and a pentameteric structure of five B-subunits of STx (STxB). Only the STxB binds to the $\mathrm{Gb}_{3}$ and therefore only the STxB was used in this study. The STxB samples A and $\mathrm{C}$ were purified by the working group of Prof. Dr. Winfried Römer (BIOSS an Institute of Biology II, Albert-Ludwigs University Freiburg) in 2013 and 2005.144 145 The STxB sample B was purified by the working group of Prof. Dr. Daniel Huster (Insti- 


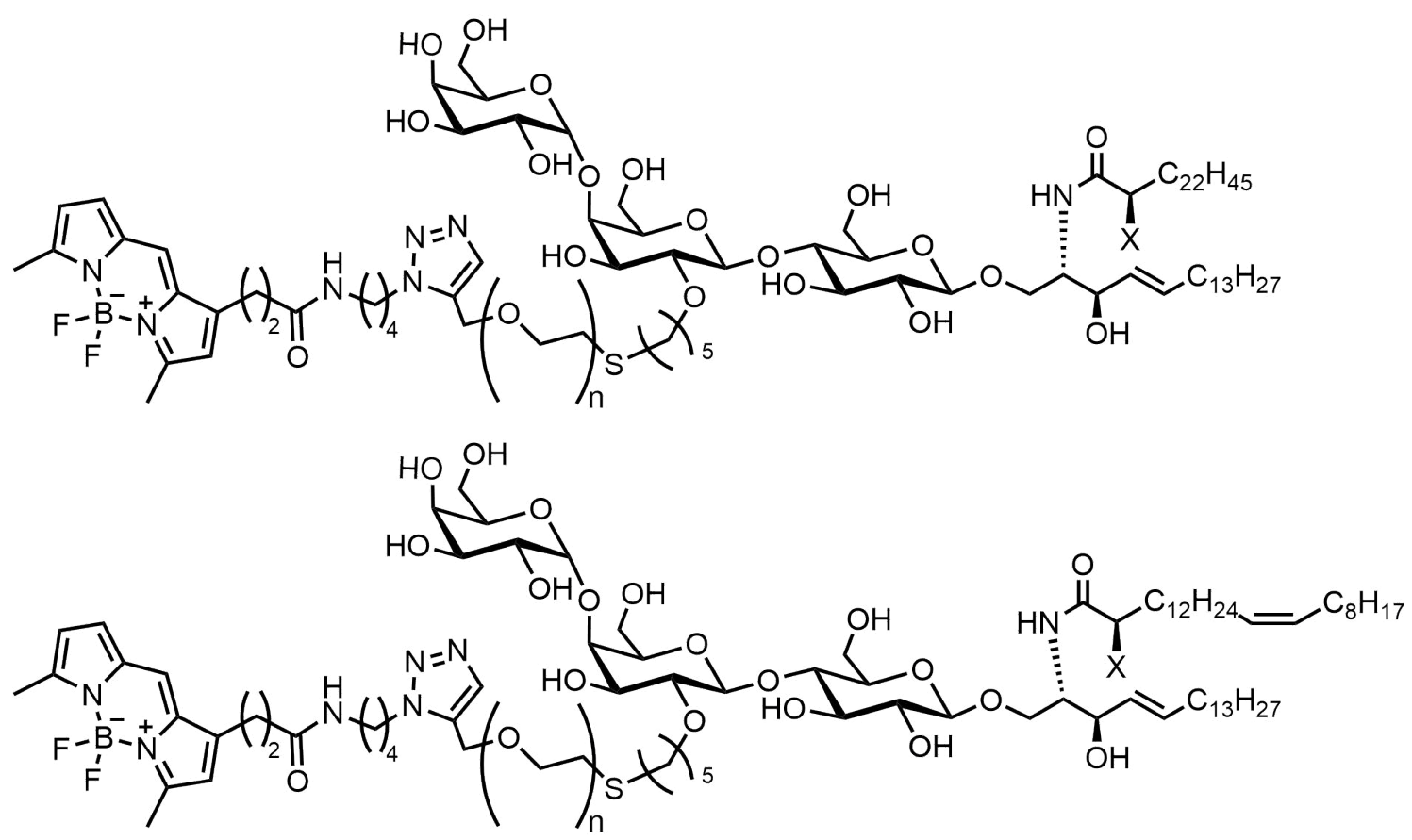

Scheme 3.12.: The structures of the head group labeled globotriaosyl ceramides $\left(\mathrm{Gb}_{3} \mathrm{~s}\right)$. The fluorophore BODIPY is connected via a PEG linker with $\mathrm{n}$ units $(3,13)$ to the 2' hydroxylgroup of the $\beta$ galactose. The fatty acid $\left(\mathrm{C}_{\mathrm{m}: \Delta}\right)$ varies in the saturated $\left(\mathrm{C}_{24: 0}\right)$ top structure and mono-unsaturated $\left(\mathrm{C}_{24: 1}\right)$ bottom structure. The $\alpha$ position of the fatty acid $(\mathrm{X})$ is hydroxylated $(\mathrm{OH})$ or not hydroxylated $(\mathrm{H})$. In total, there are eight different combination which are shorten to $\mathrm{Gb}_{3} \mathrm{PEG}_{\mathrm{n}} \mathrm{C}_{\mathrm{m}: \Delta} \mathrm{X}$.

tute for Medical Physics and Biophysics, Leipzig University) in 2017. 146 The STxB was stored at $-80^{\circ} \mathrm{C}$ and was used in a phosphate-buffered saline (PBS) buffer. The molecular weight of STxB is $7.7 \mathrm{kDa}$ and it has 69 amino acids. The protein sequence is:

TPDCVTGKVE YTKYNDDDTF TVKVGDKELF TNRWNLQSLL LSAQITGMTV TIKTNACHNG GGFSEVIFR. 50.

\subsection{Preparative methods}

The used buffers are PBS or Tris. PBS consisted of $\mathrm{KCl}(2.7 \mathrm{~mm}), \mathrm{NaCl}(136 \mathrm{~mm})$, $\mathrm{KH}_{2} \mathrm{PO}_{4}(1.5 \mathrm{~mm})$ and $\mathrm{Na}_{2} \mathrm{HPO}_{4}(8.1 \mathrm{~mm})$ and had a $\mathrm{pH}$ of 7.4 . Tris consisted of Tris $(20 \mathrm{~mm}), \mathrm{NaCl}(100 \mathrm{~mm})$ and $\mathrm{CaCl}_{2}(1 \mathrm{~mm})$ and had a $\mathrm{pH}$ of 7.4

\subsubsection{Preparation of vesicles}

For this work, vesicles of different lipid compositions at different temperatures were prepared. Vesicles with DOPC and a fluorescent lipid were incubated at $30^{\circ} \mathrm{C}$ for 
preparation of multi-lamellar vesicle (MLV) and incubated at room temperature for the preparation of large unilamellar vesicles (LUVs) and giant unilamellar vesicles (GUVs). The preparation temperature of phase-separated and $l_{\mathrm{o}}$ phase vesicles was $55^{\circ} \mathrm{C}$ for all vesicle preparations. The phase-separated vesicles composed of DOPC/ $\mathrm{SM} / \mathrm{Chol} / \mathrm{Gb}_{3} /$ fluorescent lipid and the $l_{\mathrm{o}}$ phase vesicles composed of SM/Chol/ fluorescent lipid.

\section{Multi-lamellar vesicles}

Multi-lamellar vesicles (MLVs) were prepared from a lipid film. $0.2-0.4 \mathrm{mg}$ of the lipid-mixture were dissolved in $200 \mu \mathrm{L}$ chloroform methanol solution (2:1) in a glass tube. The solvent was removed under a nitrogen stream with the preparation temperature of the lipid-mixture. The rest of the solvent was removed under reduced pressure at this temperature. The dry lipid film was stored at $4{ }^{\circ} \mathrm{C}$ for later use.

The lipid film was hydrated with $500 \mu \mathrm{L}$ of buffer and incubated for $20 \mathrm{~min}$ at the preparation temperature. After this time the film was vortexed for $30 \mathrm{~s}$ and incubated for $5 \mathrm{~min}$ at the preparation temperature. This procedure was repeated 3 times and the MLVs were used for the preparation of small unilamellar vesicles (SUVs) and LUVs.

\section{Small unilamellar vesicles}

Small unilamellar vesicles (SUVs) were prepared from MLVs with ultrasonic treatment. The MLVs solution was filled in an Eppendorf Tube and was placed in the ultra sonifier at $60 \%$ power for $30 \mathrm{~min}$. The SUVs were used within one day after preparation.

\section{Large unilamellar vesicles}

Large unilamellar vesicles (LUVs) were prepared from MLVs by extrusion. The extruders were cleaned before use with 2 times ethanol, 2 times water and 2 times buffer. The diameter of the LUVs was given by the pore diameter of the membrane which was placed in the middle of the extrusion system. The MLV solution with the lipid mixture DOPC and fluorescent lipid was loaded into a hamilton syringe and pressed 31 times through the porous membrane. The LUV preparation, for the phase-separated and $l_{\mathrm{o}}$ phase lipid-mixtures, was carried out in a heating chamber at $55^{\circ} \mathrm{C}$. The extruder was heated up for $10 \mathrm{~min}$, before the MLV solution was filled in. The solution was warmed up to $55^{\circ} \mathrm{C}$ in the heating chamber for $10 \mathrm{~min}$ and pressed 11 times through the membrane. The warm up and extrusion process was repeated 
3 times. After the extrusion, the LUV solution was filled in an Eppendorf Tube and used within one day after preparation.

\section{Giant unilamellar vesicles}

Giant unilamellar vesicles (GUVs) were prepared via electroformation. In this method, the lipid-mixture (0.1 mg in chlorophorm and methanol) was spread onto Indium Tin Oxide coated slides (ITO-slides) at the preparation temperature. The solvent was removed under reduced pressure at this temperature for 3 to $18 \mathrm{~h}$. The ITO-slides were put together with a silicon ring in the middle so that a chamber of $1.5 \mathrm{~mL}$ was build and later filled up with a succrose solution ( $298 \mathrm{mOsmol})$. The GUVs were prepared by using a sinewave voltage $\left(12 \mathrm{~Hz}, 1.6 \mathrm{~V}_{\mathrm{pp}}\right)$, either $2.5 \mathrm{~h}$ or overnight, at the preparation temperature. The GUV solution was stored in dark Eppendorf Tubes up to three days at room temperature before being used.

\subsubsection{Functionalization of porous substrates}

For all experiments, porous substrates were obtained and used from Aquamarijn Micro Filtration BV (Zutphen, Netherlands) with pore diameters of 1.2, 2.0, 3.0 and $5.0 \mu \mathrm{m}$. The top layer of the substrates was made out of $\mathrm{Si}_{3} \mathrm{~N}_{4}$ and had a thickness of $500 \mathrm{~nm}$. The support structure was composed of Si with a thickness of $900 \mu \mathrm{m}$.

\section{Mercaptoalcohol on gold}

The porous substrates from Aquamarijn were cleaned with an argon-plasma. A thin titanium layer was sputter-coated on top of the substrates $(40 \mathrm{~mA}, 60 \mathrm{~s}, 0.1 \mathrm{mbar})$. The titan is needed so that the gold stuck on the surface via an orthogonal evaporation process ( 30 to $40 \mathrm{~nm}, 3 \times 10^{-6}$ to $6 \times 10^{-6} \mathrm{mbar}, 0.2$ to $0.4 \mathrm{~nm} \mathrm{~s}^{-1}$ ). The gold evaporated substrates were stored from $2 \mathrm{~h}$ up to several days in a $1 \mathrm{~mm}$ mercaptoalcohol $n$-propanol solution. The used mercaptoalcohols were 2-mercapto-1-ethanol (2ME), 6-mercapto-1-hexanol (6MH), 8-mercapto-1-octanol (8MO), 11-mercapto-1undecanol (11MUD) and 16-mercapto-1-hexadecanol (16MHD).

\section{Plasma cleaned silicon nitride}

The porous substrates were cleaned by using argon-plasma and then hydrophilised by means of oxygen plasma. On the substrates, a gold layer was orthogonally evaporated ( 30 to $40 \mathrm{~nm}, 3 \times 10^{-6}$ to $6 \times 10^{-6} \mathrm{mbar}, 0.2$ to $0.4 \mathrm{~nm} \mathrm{~s}^{-1}$ ). The orthogonal evaporated gold substrates were incubated in a $0.5 \% 3$-aminopropyltrimethoxysilane 
methanol solution for $2 \mathrm{~min}$ to silanize the inner pore walls. After this the substrate was rinsed with ethanol and incubated with a solution of methoxy PEG succinimidyl carbonate $(20 \mathrm{mg})$ in $1.5 \mathrm{~mL}$ ethanol for $5 \mathrm{~min}$, the substrate were rinsed again with ethanol, dried under a nitrogen stream and stored in a petri dish. Before using the substrates for the preparation of PSMs, the gold layer was removed with an adhesive tape. 147

\section{Silicon oxide}

The porous substrates were cleaned with ethanol and dried under a nitrogen stream. The cleaned substrates were orthogonally evaporated with silicon monooxide (30 to $40 \mathrm{~nm}, 3 \times 10^{-6}$ to $6 \times 10^{-6} \mathrm{mbar}, 0.2$ to $0.4 \mathrm{~nm} \mathrm{~s}^{-1}$ ). After evaporation, the substrates were incubate for $1 \mathrm{~h}$ in water at $55^{\circ} \mathrm{C}$ before usage. 148

\subsubsection{Preparation of pore-spanning membranes}

The functionalized substrates were rinsed with ethanol, water and PBS. The wet substrates were placed into the measuring chamber. For temperature-controlled measurements, the chamber was made of copper and the substrate laid in a suitable gap. The chamber for the FCS measurement was made of steel and the substrate was fixed with a metal plate that was itself fixed on the side with a screw. The substrate in the interferometric scattering microscopy (iSCAT) measurements was prepared in a petri dish. The rest of the measurements were made in a petri dish and the substrate was fixed with adhesive tape. The chamber was filled up with PBS. It was heated up to $55^{\circ} \mathrm{C}$ for the temperature-controlled measurements, in the other case the preparation took place at room temperature. 60-100 $\mathrm{LL}$ prepared GUV solution was sedimented through a $5 \mathrm{~mL}$ pipette tip filled with buffer over the substrate for $2 \mathrm{~min}$. After waiting of 3-15 min, the PSMs were ready and the chamber was rinsed with buffer to remove the non-spreaded GUVs. For the iSCAT measurements, the prepared PSMs were incubated with gold nano particle (GNP), diameter of 20 or $40 \mathrm{~nm}$ for $30 \mathrm{~min}$. The prepared substrates for the iSCAT measurements were transferred into the PBS filled iSCAT measurement chamber which had two gold stripes with a thickness of $200 \mathrm{~nm}$. The substrates were also flipped for these measurements and set on top of the gold stripes. A $200 \mathrm{~nm}$ water layer was then between the membrane and the bottom of the iSCAT measurement chamber.147 


\subsection{Biophysical methods}

\subsubsection{Fluorescence microscopy}

A fluorescence microscope is a normal microscope only that the excitation light is not detected. A fluorophore absorb the excitation light and emitted light with higher wavelength which is explained by the Stokes shift. The emitted light is collected and filtered to be sure that no reflective light will be measured by the fluorescence microscope.

The wavelength of the excitation light can be adjusted by an optical filter and a white light source (mercury vapour lamp) or a light source which has a smaller wavelength spectrum, such as light-emitting diodes (LEDs) or lasers. The filtered excitation light was focused with an objective into the sample. The fluorophore absorb the light and emitted light with higher wavelength which was collected by the same objective and detected by a camera, photodiode or photomultiplier.

\section{Confocal laser scanning microscope}

A confocal laser scanning microscope (CLSM) works with lasers as excitation light source. The laser is scanned over the probe with two movable mirrors, pointwise excitation. The emitted light passes a pinhole to set the confocality of the system and is detected with a photomultiplier tube. In this work three different CLSMs were used. The CLSM from OLYMPUS was used for the fatty acid $\mathrm{Gb}_{3}$ phase distribution study and the phase separation on PSMs study. Two different CLSMs from ZEISS (LSM 710, LSM 880) were used for the head group labeled $\mathrm{Gb}_{3}$ phase distribution study. The LSM 880 was also used for the FCS measurements.

\section{Fluorescence correlation spectroscopy}

To measure fluorescence correlation spectroscopy (FCS), a CLSM can be used. Only one point spread function (PSF) is measured over the time. The PSF intensity $(I(x, y, z))$ is an ellipsoid with the short half axis $\omega_{1}$ in $x$ - and $y$-directions and $\omega_{2}$ in the $z$-direction as long half axis

$$
I(x, y, z)=I_{0} \exp \left(-\frac{2\left(x^{2}+y^{2}\right)}{\omega_{1}^{2}}-\frac{2 z^{2}}{\omega_{2}^{2}}\right) .
$$




\section{Theory}

The intensity $(I(t))$ at time $(t)$ of the PSF is compared to the intensity $(I(t+\tau))$ with the lag time $(\tau)$. The auto-correlation $(G(\tau))$ is calculated on the two different intensities by changing the lag time. 149

$$
G(\tau)=\frac{\langle I(t+\tau) \cdot I(t)\rangle}{\langle I(t+\tau)\rangle \cdot\langle I(t)\rangle}
$$

The mean intensities $(\langle I()\rangle)$ of the two different intensity time traces are the same:

$$
\langle I(t+\tau)\rangle=\langle I(t)\rangle
$$

With this, the auto-correlation function is reduced to:

$$
G(\tau)=\frac{\langle I(t+\tau) \cdot I(t)\rangle}{\langle I(t)\rangle^{2}}
$$

The FCS curves are calculated by the intensity fluctuations $(\delta I(t))$ and not by the absolute intensity. The fluctuation is calculated by the difference of the mean intensity from the intensity time trace.

$$
\delta I(t)=I(t)-\langle I(t)\rangle
$$

The intensity fluctuation in the auto-correlation results in

$$
G(\tau)=\frac{\langle(\langle I(t)\rangle+\delta I(t+\tau)) \cdot(\langle I(t)\rangle+\delta I(t))\rangle}{\langle I(t)\rangle^{2}}
$$

and the factorizing of this resulted function gives

$$
G(\tau)=\frac{\langle\langle I(t)\rangle\langle I(t)\rangle+\langle I(t)\rangle \cdot\langle\delta I(t+\tau)\rangle+\langle I(t)\rangle \cdot\langle\delta I(t)\rangle+\delta I(t+\tau) \cdot \delta I(t)\rangle}{\langle I(t)\rangle^{2}} .
$$

The equation 3.7 is reduced by the fact that the mean values of the fluctuation are zero.

$$
G(\tau)=1+\frac{\langle\delta I(t+\tau) \cdot \delta I(t)\rangle}{\langle I(t)\rangle^{2}}
$$

The 3D-diffusion auto-correlation function is generated from the intensity profile of the PSF (equation 3.1) and by the assumption that the intensity fluctuation is only affected by the diffusion of particles. The 3D-diffusion auto-correlation function 
is

$$
G(\tau)=1+\frac{\gamma}{N}\left(\frac{1}{1+\tau / \tau_{d}}\right)\left(\frac{1}{1+\left(\omega_{1} / \omega_{2}\right)^{2}\left(\tau / \tau_{d}\right)}\right)^{0.5},
$$

with $\gamma$ as a correction factor for the intensity profile in the focus 150 and the fit to a measured auto-correlation function resulted in the diffusion time $\left(\tau_{d}\right)$. $149|151| 152$

The diffusion from lipids in membranes takes only place in $2 \mathrm{D}$ and not $3 \mathrm{D}$, so the 3D auto-correlation is reduced by reducing the PSF from an ellipsoid to a plane orthogonal to the optical axis. 150

$$
G(\tau)=1+\frac{\gamma}{N}\left(\frac{1}{1+\tau / \tau_{d}}\right)
$$

The diffusion constant $(D)$ of the lipids is calculated from the 2D-diffusion time $\left(\tau_{d}\right)$ with

$$
\tau_{d}=\frac{\omega_{1}^{2}}{4 D}
$$

This equation is only correct if the PSF is known and if the membrane is in the mid-plane of the PSF. Then the short half axis of the PSF $\left(\omega_{1}\right)$ is equal to the radius of the cylinder. To overcome this specification, Z-scan FCS can be performed. Z-scan FCS uses the fact that the diffusion time depends quadraticaly on the Z-position of the PSF.150

$$
\tau_{d}(\Delta z)=\frac{\omega_{1}^{2}}{4 D}\left(1+\frac{\lambda_{0}^{2} \Delta z^{2}}{\pi^{2} n^{2} \omega_{1}^{4}}\right)
$$

The diffusion constant $(D)$ and the short half axis of the PSF $\left(\omega_{1}\right)$ can now be calculated from a quadratic fit of the different calculated diffusion times $\left(\tau_{d}\right)$ at different Z-positions $\left(\Delta z=z-z_{0}\right)$. $z_{0}$ is equal to the minimum of the fitting curve. The laser wavelength $\left(\lambda_{0}\right)$ and the reflective index of the solution $(n)$ is set up. 150 The short half axis of the PSF $\left(\omega_{1}\right)$ can also be controlled by the numbers of diffusion particles in dependency of the Z-position with the averaged concentration of diffusion fluorescent molecules $(c)$.

$$
N(\Delta z)=\pi c \omega_{1}^{2}\left(1+\frac{\lambda_{0}^{2} \Delta z^{2}}{\pi^{2} n^{2} \omega_{1}^{4}}\right)
$$

\section{The measurement and calculation of the diffusion coefficient with FCS}

The auto-correlation function at different Z-positions are measured and the equation 3.12 can be fit with different diffusion times and different Z-positions.

The use of an upright fluorescence microscope has the disadvantage that the mechanical focus drift cannot be corrected by a stabilized focus which measures the 
reflection beam of a second laser at the cover slide. The mechanical focus drift must be inhibited. This was done by using a metal measurement chamber.

To increase the reliability of the measurement, the auto-correlation was measured five times for $2 \mathrm{~min}$ at each Z-position. The fit of the 2D auto-correlation function (equation 3.10 is weighted with the errors of the lag time $(\sigma(\tau))$

$$
\sigma(\tau)=\sqrt{\frac{1}{L(L-1)} \sum_{l=1}^{L}\left(\frac{G_{l}(\tau)-G_{l}(\infty)}{G_{l}(0)-G_{l}(\infty)}-\frac{1}{L} \sum_{l=1}^{L} \frac{G_{l}(\tau)-G_{l}(\infty)}{G_{l}(0)-G_{l}(\infty)}\right)^{2}} .
$$

$L$ describes the number of measurements at the same position with the index $(l)$. $G_{l}(\infty)$ and $G_{l}(0)$ are estimated by averaging of long and short delay times respectively. $\frac{150}{15}$ This calculated error is used to get a precisely fitted 2D-diffusion autocorrelation with the $\chi^{2} \operatorname{method} 153$

$$
\chi_{\text {Autocorrelation }}^{2}=\sum_{i}\left(\frac{G\left(\tau_{i}\right)-\overline{G\left(\tau_{i}\right)}}{\sigma\left(\tau_{i}\right)}\right)^{2} .
$$

The $\chi^{2}$ evaluation is also used for the Z-scan FCS fit (equation 3.12 with the error of the auto-correlations fit $\left(\chi_{\text {Autocorrelation }}^{2}\right)$ at the different Z-positions $(\Delta z)$.

$$
\chi_{\mathrm{Z} \text {-scan FCS }}^{2}=\sum_{i}\left(\frac{\tau_{d}\left(\Delta z_{i}\right)-\overline{\tau_{d}\left(\Delta z_{i}\right)}}{\chi_{\text {Autocorrelation }}^{2}\left(\Delta z_{i}\right)}\right)^{2}
$$

The diffusion constant $(D)$ is calculated from the Z-scan FCS fit (equation 3.12).

\subsubsection{Interferometric scattering microscopy}

Interferometric scattering microscopy (iSCAT) was used to determine the diffusion constants from the f-PSM and solid supported PSM (s-PSM) on the $6 \mathrm{MH}$ on gold $(6 \mathrm{MH}-\mathrm{Au})$ and plasma cleaned silicon nitride $\left(\mathrm{p}-\mathrm{Si}_{3} \mathrm{~N}_{4}\right)$ functionalized substrates. The measurements were performed by Dr. Susann Spindler in the group of Prof. Dr. Vahid Sandoghdar (Max Planck Institute for the Science of Light, Erlangen). The analyzed lipids (1,2-dioleoyl-sn-glycero-3-phosphoethanolamine-Ncap-biotinyl (cb-DOPE)) were labeled with GNPs. The lipid GNP connection was over a biotin streptavidin binding. Light was reflected not only by the GNP but also from the cover slide and the porous substrate. The reflected light from the GNP interferes with the reflected light from the substrate or cover slip. The light intensity was measured with a camera. The position of the GNP was shown by a 2D-Gausion curve in the light intensity images. This was fitted and the position of the GNPs were 
connected to a trajectory. The diffusion constant was calculated with the covariancebased estimation (CVE), this had the advantage that the fitting errors of the GNP position were considered. 154

\section{Preparation of the iSCAT measurement}

The PSMs were prepared as previous described (section 3.2.3), with PBS in a petri dish. The prepared PSMs were incubated with the 20 or $40 \mathrm{~nm}$ GNPs for $30 \mathrm{~min}$. Then the substrate was transferred to the measured chamber, filled with PBS. This camber had two gold spacers with a thickness of $\sim 200 \mathrm{~nm}$. The flipped substrate were placed, so that between the membrane and the cover slide a buffer film of $200 \mathrm{~nm}$ were. Thus substrates were measured at a home build inverse microscope in Erlangen. 147 The membrane patch were found with the microscope and the ATTO532-1,2-dioleoylsn-glycero-3-phosphoethanolamin (ATTO532-DOPE) fluorophore. The length of one video was $5 \mathrm{~s}$ by a frame rate of $1 \mathrm{kHz}$.

\subsubsection{Atomic force microscopy}

An atomic force microscopy (AFM) uses a cantilever to detect height differences in the $\mathrm{nm}$ regime or forces in the $\mathrm{pN}$ regime. A cantilever can be described with Hooke's law

$$
F=k \Delta x
$$

The measured force $(F)$ is proportional to the cantilever stiffness $(k)$ and the deflection of the cantilever $(x) .149$ The deflection of the cantilever is in the sub nm regime. The accuracy of the small deflections from the cantilever is achieved by reflecting a laser beam from the backside of the cantilever onto a 4-quadrant diode.

AFM can be used for surface topography images or force measurements. The quantitative imaging (QI) mode was used in this work to measure a surface topography from the PSMs. The principle of this mode is to measure a force distance curve up to the maximum force setting and than retract the cantilever to a set distance length at each pixel. The advantage of this mode is that shear forces which can be set to scanning the probe are minimized, because the cantilever indents the probe only from the top and not from the side.

\section{Measurement of membrane tension}

The membrane tension of PSMs was measured by AFM. PSMs were prepared, as previously described (section 3.2.3), with PBS in a petri dish and fixed with an 
adhesive tape. The center of the microscope images and the center of the topographic images were aligned by using a home-build calibration cross. A membrane patch was found with the fluorescence microscope and a QI topography image was taken with the AFM of the patch. f-PSMs were identified in the QI images. At the center of the f-PSMs, new force indentation curves were taken, to calculate the membrane tension.

The membrane tension of the f-PSM was extracted from the force indentation curves by fitting

$$
F=2 \pi R \sigma_{\mathrm{z}} \frac{u^{\prime}(R)}{\sqrt{1+u^{\prime}(R)^{2}}} .
$$

The measured force $F$ depends on the radius of the f-PSM $(R)$. The membrane surface geometry $(u(R))$ is calculated from the indentation depth $(z)$. The membrane tension $(\sigma)$ is extracted from

$$
\sigma_{z}=\sigma+K_{\mathrm{A}} \frac{\Delta A}{A_{0}}
$$

The increased membrane area $\left(\triangle A / A_{0}\right)$ multiplied with the area compressibility modulus $\left(K_{\mathrm{A}}\right)$ affected the $\sigma$ upon the indentation. This fitting neglects the bending of the membrane, because the pore radius is greater than the membrane thickness by a factor of $>100.155$

\subsubsection{Surface plasmon resonance}

Surface plasmon resonance (SPR) is used in many applications in order to analyze molecular interactions. In this work, the dissociation constant $\left(K_{\mathrm{d}}\right)$ of $\mathrm{STxB}_{\mathrm{Gb}}$ was measured with SPR. To measure the binding, the receptor molecule $\left(\mathrm{Gb}_{3}\right)$ is fixed to a sensor surface. The binding partner $(\mathrm{STxB})$ is rinsed over the surface. The change of refractive index near the surface is time-dependently detected and reflects the binding of $\mathrm{STxB}$ to $\mathrm{Gb}_{3}$.

The refractive index near the surface is detected via the surface plasmon resonance frequencies. The surface plasmon are stimulated by a total reflected laser beam in a certain angle to the optical axis. By changing the angle of the laser beam the intensity of the surface plasmon changes and the reflective light of the laser varied. At the specific angle which represents the surface plasmon resonance frequencies the reflective light is at a minimum. The change of the reflective index near the surface changes the surface plasmon resonance frequencies, which changes the angle of the minimum from the reflected laser light. This change in the angle was measured time dependent. 149 


\section{The SPR experiment}

$40 \mathrm{~nm}$ gold coated SPR chips were incubated in a $1 \mathrm{~mm}$ octanthiol solution over night to generate a hydrophobic self-assembled monolayer (SAM). A SPR chip was set up into the system and the measurement chambers were rinsed with buffer. The system had two different channels, the analytic and control channel. To avoid systematical errors like temperature fluctuation, the reflective units from both channels were measured and subtracted $\Delta R U$, fist correction.

SUVs with the lipid composition DOPC/ $\mathrm{Gb}_{3 \text { porc }}$ (95:5) were rinsed only over the analytic channel for $30 \mathrm{~min}$. The reflective index changed by spreading the SUVs on the functionalized SPR chips which is seen by the increase of the $\Delta R U$ in figure $3.1 \mathrm{~A}$ starting at the injection 1. The control SUVs composed of DOPC were rinsed overboth channels, the analytic channel and the control channel, for $30 \mathrm{~min}$. The possible spreading of the SUVs is seen by a decrease of the $\Delta R U$, because the reflective index of the control channel changes due to the fact that the vesicles spread to the surface (figure $3.1 \mathrm{~A}, 2$ ). To remove multi lamellar membrane stacks, a $50 \mathrm{~mm} \mathrm{NaOH}$ solution was rinsed over both channels five times for 2 min (figure 3.1 A, 3-7). After removing the multi lamellar membrane stacks, the surface of the SPR chip is full of a membrane monolayer. In the analytic channel, the receptor lipid was incorporated into the membrane on the surface. In the control channel, a DOPC membrane was spread on the surface.
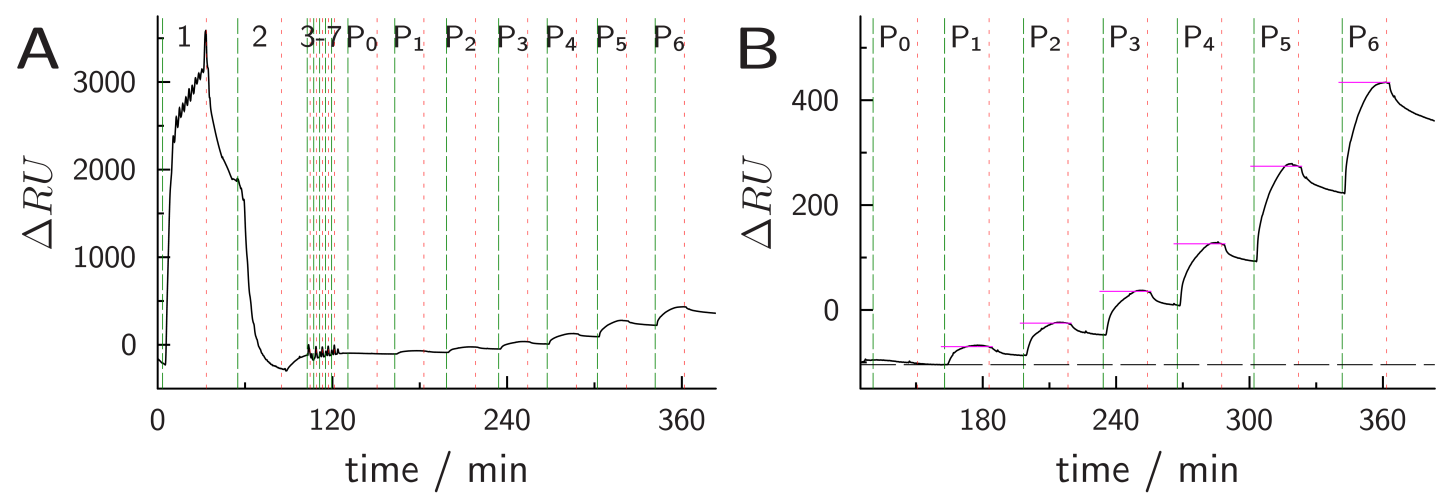

Figure 3.1.: A complete surface plasmon resonance (SPR) measurement with the different injection (1-7 and $\left.\mathrm{P}_{0-6}\right)$. The reflective index of the analytic channel was corrected by the control channel $(\triangle R U)$ and plotted again the time. The complete measurement is shown in A) with the insertion of the analytic vesicles (1), the control vesicles (2) and five steps of rinsing with $\mathrm{NaOH}(3-7)$. The addition of different protein concentration $\left(\mathrm{P}_{0-6}\right)$ starting with a control, adding only buffer $\left(\mathrm{P}_{0}\right)$. In $\mathrm{B}$, a zoom into the protein addition is shown and the cumulative analysis is illustrated by the horizontal black line and the magenta lines in the different protein additions, the differences are the measured values. 
The measurement starts with a control, only buffer was rinsed over the prepared membrane with the same settings as protein injection, to see the influence of the injection (figure 3.1. $\mathrm{P}_{0}$ ). Different protein concentrations were rinsed afterwards over the membrane, starting with the lowest one (figure 3.1, $\mathrm{P}_{1}-\mathrm{P}_{6}$ ).

The analysis of the dissociation constant can be done with the second correction method. The buffer injection will be substracted from each protein injection and plotted together. For this analysis, it must be clear that the protein will fully take off from the surface, but this is not the case for $\mathrm{STxB} \mathrm{Gb}_{3}$ binding. To overcome the not $100 \%$ take off of the protein, the SPR time trace was cumulatively analyzed. The values before the first protein injection were the reference $\Delta R U$ (figure 3.1 $\mathrm{B}$, black broken line). The difference from the reference $\Delta R U$ to the $\Delta R U$ at the end of each protein measurement (figure 3.1 $\mathrm{B}$, cyan line in $\mathrm{P}_{1}-\mathrm{P}_{6}$ ) is the reflecticity change and can be fitted with the protein concentrations to a Langmuir adsorption isotherm

$$
R_{r e l}=\frac{R_{r e l, \max } c(\mathrm{STxB})}{K_{\mathrm{d}}+c(\mathrm{STxB})}
$$

in order to calculate the dissociation constant $\left(K_{\mathrm{d}}\right)$. To compare different measurements, the relative reflectivity change was calculated by normalizing to a protein concentration of $300 \mathrm{nM}, R_{\text {rel }}=1$.

\subsection{Data evaluation}

\subsubsection{Phase distribution in GUVs}

In this work, the phase preference of fluorescently labeled $\mathrm{Gb}_{3} \mathrm{~s}$ was measured and analyzed (results in chapter 4.2.2 and 4.2.3). Phase-separated GUVs were prepared as described in chapter 3.2.1. $60-100 \mu \mathrm{L}$ of prepared GUV solution was sedimented through a $5 \mathrm{~mL}$ pipette tip filled with PBS into a bovine serum albumin (BSA) passivated petri dish for 2 min. Afterwards, GUVs were measured with the CLSM. A Z-stack was measured from the GUVs and two fluorophores are recorded in separated channels. One channel represents the fluorescence intensity of the labeled $\mathrm{Gb}_{3} \mathrm{~s}$ and the second channel shows the fluorescence intensity of the $l_{\mathrm{d}}$ phase marker (Dy731DOPE). 141

The analysis of the labeled $\mathrm{Gb}_{3}$ distribution in phase-separated GUVs was done with a custom written Matlab script. The measured Z-stacks were measured at different CLSMs with different data structures. These data structures were aligned so that there was no need to rewrite the Matlab script for the different CLSMs. 

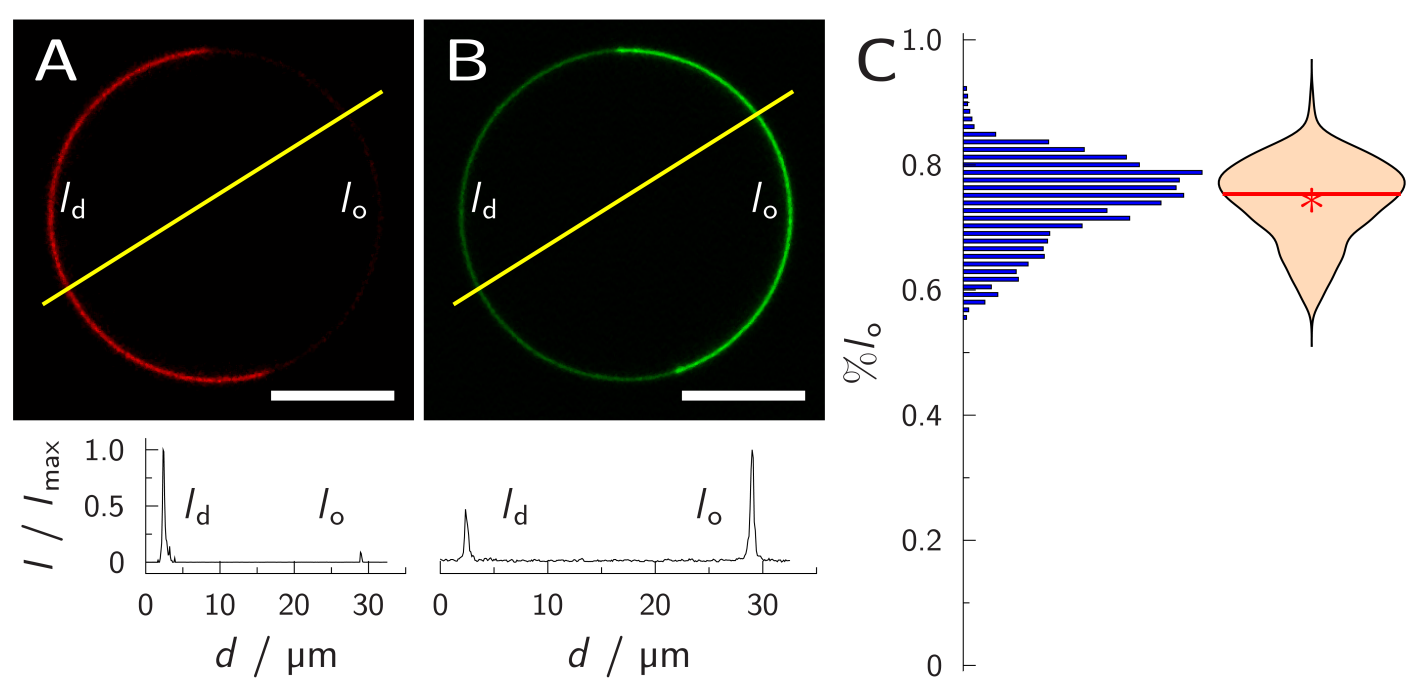

Figure 3.2.: Confocal images of a phase-separated GUV composed of DOPC/ $\mathrm{SM}_{\text {porc }} /$ Chol/ $\mathrm{Gb}_{3} \mathrm{PEG}_{13} \mathrm{C}_{24: 0} \mathrm{H} /$ Dy731-DOPE (39:39:20:1:1). A) Dy731-DOPE fluorescence (red); B) $\mathrm{Gb}_{3} \mathrm{PEG}_{13} \mathrm{C}_{24: 0} \mathrm{H}$ fluorescence (green). The yellow lines indicate where the fluorescence intensity profiles (bottom images) have been obtained. From the intensity profiles, liquid ordered phase distribution $\left(\% l_{\mathrm{o}}\right)=0.682$ was calculated for $\mathrm{Gb}_{3} \mathrm{PEG}_{13} \mathrm{C}_{24: 0} \mathrm{H}$. C) Histogram and corresponding violin plot obtained from 60 GUVs with 2525 individual analyzed profile lines with the composition as in A. The red solid line indicates the median value, the red star the mean value. Scale bar $10 \mu \mathrm{m}$.

To achieve an unbiased data evaluation, the profile lines of each GUV slide from the GUV stacks was set manually only in the phase marker channel (Dy731-DOPE, figure 3.2 A) Every profile line crosses the GUV twice. One time in the $l_{\text {o }}$ phase and the second time in the $l_{\mathrm{d}}$ phase, and it crosses the center of the GUV to overcome polarization effects (figure 3.2 yellow line). These profile lines were analyzed in both channels of the same GUV slide (figure 3.2 A and B). The intensity $(I(d))$ of the profile line was fitted against the position $(d)$ of the profile line with a two-peak Gaussian fit, each peak for one membrane crossing.

$$
I(d)=a_{1} \exp \left(\left(\frac{d-b_{1}}{c}\right)^{2}\right)+a_{2} \exp \left(\left(\frac{d-b_{2}}{c}\right)^{2}\right)+e
$$

The fit parameter $a_{1}, a_{2}$ describes the amplitude or maximum intensity of the peaks, $b_{1}, b_{2}$ the position of the peaks, $c$ the peak width which is equal for both peaks, because the membrane thickness around the GUV is the same. $e$ describes the background intensity. The peak with the maximum intensity from the Dy731-DOPE profile represents the $l_{\mathrm{d}}$ phase peak. This was used to select the phases in the analyzed labeled $\mathrm{Gb}_{3}$ intensity profile in order to calculate the $l_{\mathrm{o}}$ phase distribution $\left(\% l_{\mathrm{o}}\right)$. The ratio between $I\left(l_{\mathrm{o}}\right)$ and the total intensity of both phases allows the determination of the 
proportion of labeled $\mathrm{Gb}_{3}$.

$$
\% l_{\mathrm{o}}=\frac{I\left(l_{\mathrm{o}}\right)}{I\left(l_{\mathrm{o}}\right)+I\left(l_{\mathrm{d}}\right)}
$$

This analysis was done for several GUVs. The preparation was also done on different preparation days. To see the distribution of the $\% l_{o}$, the data was plotted in a histogram and for a better comparison it was also plotted as a violin plot (figure $3.2 \mathrm{C}$ ).

For the statistical analysis of the labeled $\mathrm{Gb}_{3} \mathrm{~S}$ (chapter A.7), a linear mixed-effects model (LMM) was fitted to the whole $\% l_{\mathrm{o}}$ data

$$
\% l_{\mathrm{o}}=X \beta+Z u+\epsilon
$$

in order to evaluate the effects of the different chemical properties of the labeled $\mathrm{Gb}_{3} \mathrm{~s}$. The effects of the measured $\% l_{\mathrm{o}}$ of a phase-separated GUV are listed in the vectors of fixed effects $\beta$ and the random effects $u$. The fixed effects describe the effect of the different chemical properties from the labeled $\mathrm{Gb}_{3}$ such as the different fatty acids, to the $\% l_{0}$. The random effects describe systematic distributions such as the influence of the GUVs population from each day to the $\% l_{\mathrm{o}}$ or the distribution of the $\% l_{\mathrm{o}}$ in one GUV. The description to which group the measured data belongs is listed in the coefficient matrix of the fixed effect $X$ and random effect $Z$. At least, a vector of random error $(\epsilon)$ was added.

Equation 3.23 was solved with the maximum likelihood estimation which optimizes the equation in the way that the different random effects as well as the random error are normally distributed with a mean value of zero. The results and the different LMM equations are summarized in chapter A.7.

\subsubsection{Phase separation on PSMs}

In this thesis, phase separation of PSMs was measured and analyzed in dependence of temperature and lipid mixture. The results of this study can be seen in chapter 5.2 . The lipid mixture varied in the Chol content from 0 to $50 \mathrm{~mol} \%$ in a DOPC/SM 1:1 matrix. The measurement was performed with a CLSM within a temperaturecontrol chamber. ${ }^{156}$ The prepared PSMs (chapter 3.2.3) on top of a silicon oxide $\left(\mathrm{SiO}_{\mathrm{x}}\right)$ functionalized substrate were heated and cooled between 25 and $55^{\circ} \mathrm{C}$. One measurement series was done on one membrane patch, either heated or cooled. The series consists of several images taken at certain temperatures. Different series were measured on one substrate with the same Chol concentration in the GUV solution. 
The taken images are composed of two different channels. The channels display the intensity of the phase markers BODIPY-Chol and TexasRed-DHPE. There are two possibilities of phase separations located in the PSM within the measured phase diagram (figure 3.3). Firstly, the PSM phase-separated into the gel like $\left(l_{\beta}\right) / l_{\mathrm{d}}$ phase at low temperature and, at high temperature, it is homogeneous in the $l_{\mathrm{d}}$ phase. Secondly, at low temperature, the phase separation is between the $l_{\mathrm{o}} / l_{\mathrm{d}}$ phase. The phase separation was analyzed by eye for each series, comparing the images with the illustration in figure 3.3 .

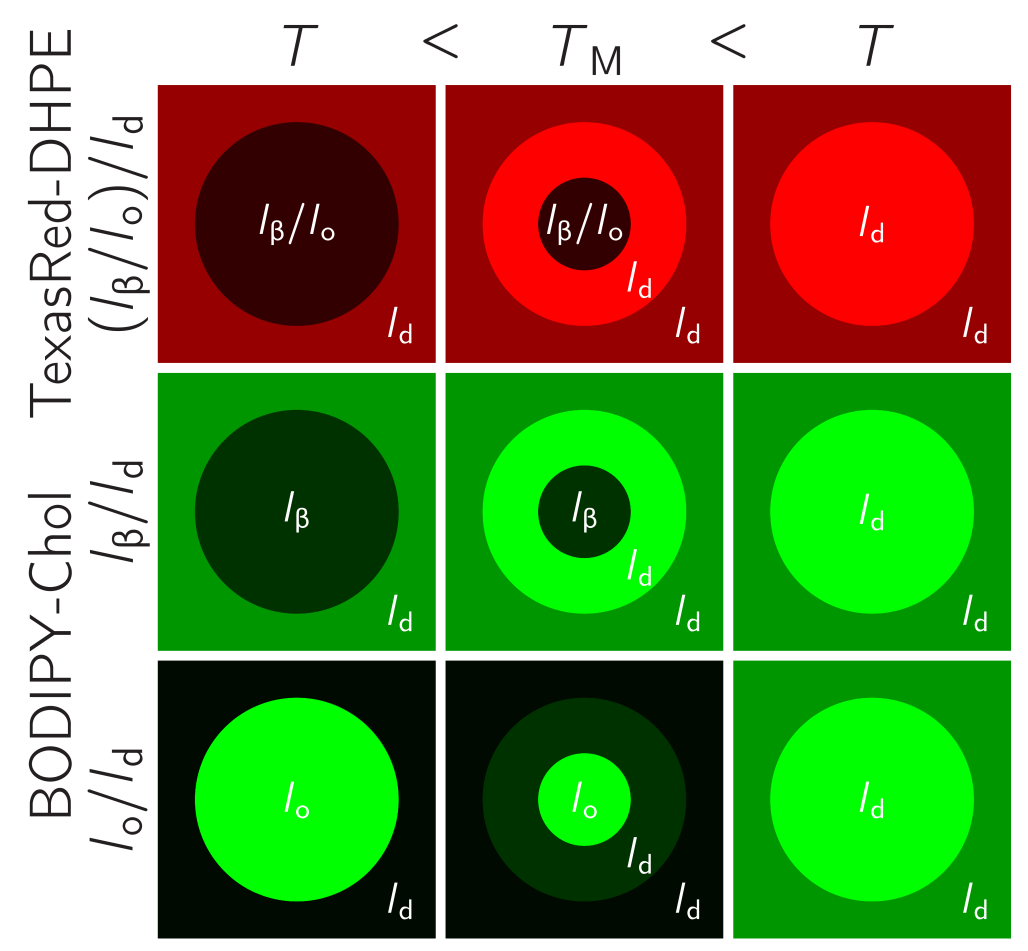

Figure 3.3.: The two different phase separations are illustrated with the different intensities of the two different fluorophores sulforhodamine-1,2-dihexanoyl-sn-glycero-3phosphoethanolamine (TexasRed-DHPE) (red, upper line) and 23-(dipyrrometheneboron difluoride)-24-norcholesterol (BODIPY-Chol) (green, bottom lines) in the pore-spanning membranes (PSMs). The membrane is phase separated at temperature lower than the transition temperature $\left(T_{\mathrm{M}}\right)$ in the gel like $\left(l_{\beta}\right)$ /liquid disordered $\left(l_{\mathrm{d}}\right)$ or liquid ordered $\left(l_{\mathrm{o}}\right) / l_{\mathrm{d}}$ phase separation. At higher temperature than the $T_{\mathrm{M}}$ the PSM is homogeneous in the $l_{\mathrm{d}}$ phase.

In the measurements and the illustration, the channel of TexasRed-DHPE is not influenced by the type of phase separation. It represents the $l_{\mathrm{d}}$ phase, which is at lower temperature as the transition temperature $\left(T_{\mathrm{M}}\right)$ always in the s-PSM and at higher temperature homogeneously distributed in the PSM. Therefore, this channel was used to identify the $T_{\mathrm{M}}$ of each series. 
In the first step, each fluorescence micrograph from all the temperature series and lipid compositions was separately analyzed to extract the intensity ratio of the f-PSM to the s-PSM. This was done with a custom designed Matlab-script. The analysis of the image sequence was performed in random order to avoid systematical errors from the user. The fluorescently micrograph analysis is schematically shown in scheme 3.13 .

The two channels of the fluorescence micrograph show the fluorescence intensity of BODIPY-Chol and TexasRed-DHPE (scheme 3.13). To divide the fluorescence micrograph into the pore and rim regions, the channels were merged and smoothed with a median filter. The pore localization in the merged fluorescence micrograph was automated. Pores were not always identified as a circular object. Unidentified pores were extrapolated with the conditions of hexagonal pore orientation and equidistant spacing between the pores (scheme 3.13). As a control of the algorithm, the result was checked by visual judgment. If the calculated pore mask did not fit into the merged image (bad alignment), some pore centers had to be set manually, while the rest were extrapolated (scheme 3.13). The well aligned pore mask was used to separate the regions of the pores and the rim. The membrane areas (f-PSM and s-PSM) in both regions were found by threshold analysis. For that, an intensity histogram of the pore region or the rim region was created in order to have a decision guide for the threshold setting. Each pixel with an intensity above the threshold belongs to the membrane area, whereas each pixel with an intensity below the threshold belongs to the non membrane region, the so-called background. The generation of the f-PSM mask was shape constrained, because pores can only be completely spanned by membrane or not. This condition was realized with a filling parameter. If the amount of pixels which were higher than the threshold was greater than the filling parameter, the whole pore was included in the f-PSM mask, and if not the whole pore was included in the background mask. The mean intensity $(I)$ of the TexasRed-DHPE channel was read out for the different areas by using these masks (f-PSM, Background, s-PSM). Afterwards, the intensity ratio

$$
\frac{I_{\mathrm{f}-\mathrm{PSM}}}{I_{\mathrm{s}-\mathrm{PSM}}}=\frac{I_{\mathrm{f}-\mathrm{PSM}}-I_{\text {Background }}}{I_{\mathrm{s}-\mathrm{PSM}}-I_{\text {Background }}} .
$$

was calculated from the mean intensities of the TexasRed-DHPE channel.

The calculated intensity ratio of one series were plotted against the temperature $(T)$ from the measured temperature ramp (figure 3.14). The $T_{\mathrm{M}}$ is the tuning point of a sigmoidal curve, 104 which was fit with 


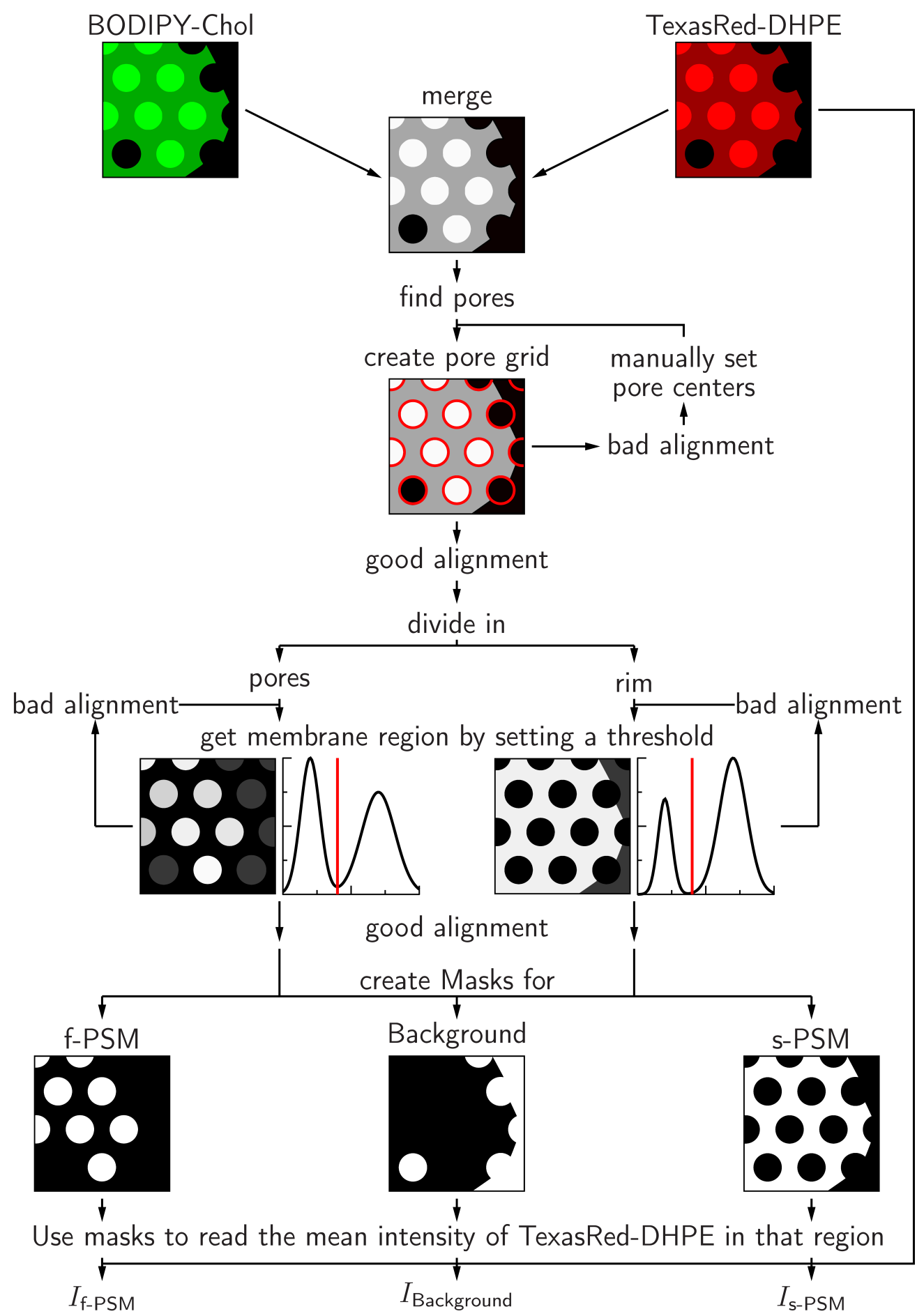

Scheme 3.13.: The process chart for the image intensity calculation. The two channels of the fluorescence image were merged to calculate the pore mask. The pore and rim regions were extracted and separately analyzed to get the masks of the membrane areas (freestanding pore-spanning membrane (f-PSM), solid supported pore-spanning membrane (s-PSM)). The non membrane areas were collected and assigned to the background. These masks were utilized to gain the mean fluorescent intensities of the specific regions from the TexasRed-DHPE fluorescence image $\left(I_{\mathrm{f}-\mathrm{PSM}}, I_{\text {Background }}\right.$ and $\left.I_{\mathrm{s}-\mathrm{PSM}}\right)$. 


$$
\frac{I_{\mathrm{f}-\mathrm{PSM}}}{I_{\mathrm{S}-\mathrm{PSM}}}(T)=I_{\mathrm{end}}+\frac{I_{\text {start }}-I_{\mathrm{end}}}{1+\exp \left(\frac{T-T_{\mathrm{M}}}{d T}\right)} .
$$

The width of the transition $(d T)$ describes the transition temperature range. The intensity ratio at low temperature is described through $I_{\text {start }}$ and the intensity ratio at high temperature is $I_{\text {end }}$.

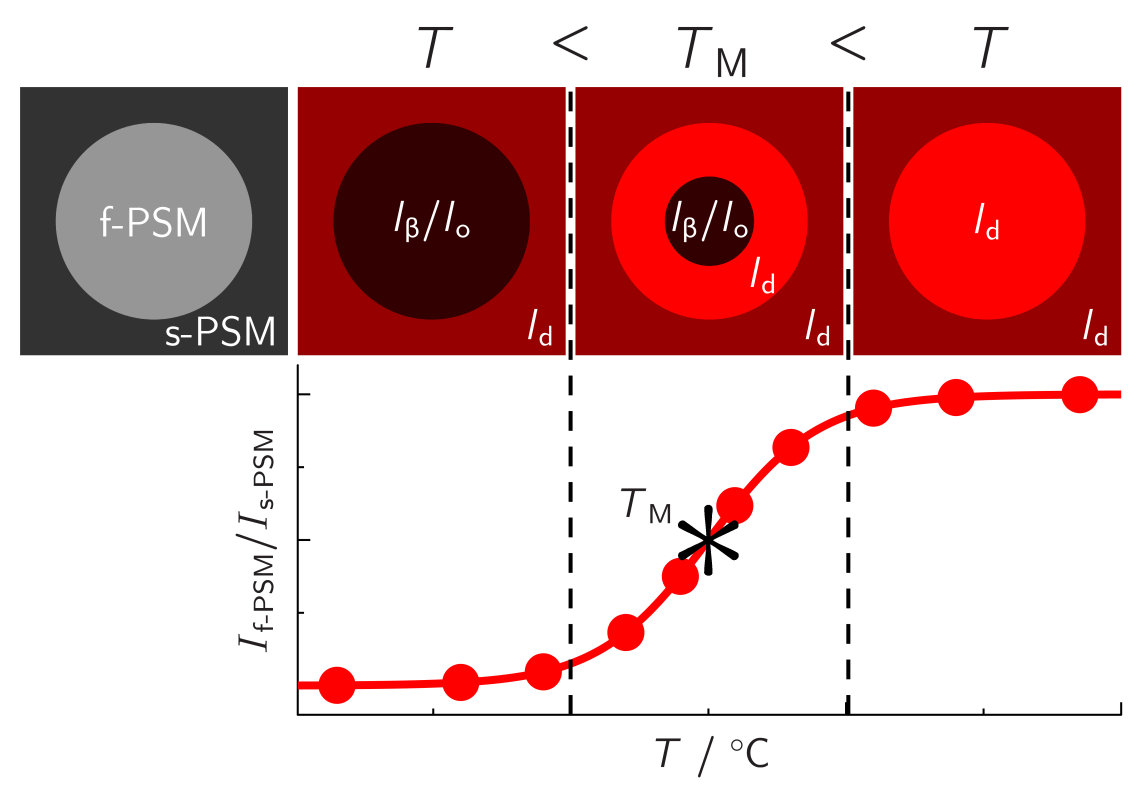

Scheme 3.14.: A schematically illustration of how to get the transition temperature $\left(T_{\mathrm{M}}\right)$ of a temperature series form pore-spanning membranes (PSMs). The intensity ratio $I_{\mathrm{f}-\mathrm{PSM}} / I_{\mathrm{s}-\mathrm{PSM}}$ is plotted against the temperature. The turning point is the $T_{\mathrm{M}}$. The intensity ratio of the PSMs at three temperatures is illustrated above as fluorescence micrograph.

The measured $T_{\mathrm{M}}$ values of all samples with equal Chol concentration were averaged and plotted against the Chol concentration, as phase diagram. 


\section{Labeled globotriaosyl ceramides $\left(\mathrm{Gb}_{3} \mathrm{~s}\right)$ and their phase behavior in phase-separated membranes}

In this thesis, the phase preference of the receptor lipid globotriaosyl ceramide $\left(\mathrm{Gb}_{3}\right)$ was analyzed before shiga toxin (STx) binding. This aspect was investigated using phase-separated giant unilamellar vesicles (GUVs) and fluorescently labeled $\mathrm{Gb}_{3} \mathrm{~s}$. The B-subunit of STx (STxB) binds the natural $\mathrm{Gb}_{3}$ in the liquid ordered $\left(l_{\mathrm{o}}\right)$ phase of phase-separated membranes. .54 Furthermore, the STxB binding preference was tested for the fluorescently labeled $\mathrm{Gb}_{3} \mathrm{~s}$. $\mathrm{Gb}_{3} \mathrm{~s}$ in cell membranes differ in fatty acid length, saturation and $\alpha$-hydroxylation. Different fatty acids were analyzed to observe the influence of the fatty acids on the phase preference of the $\mathrm{Gb}_{3} \mathrm{~s}$ and the binding preferences of STxB.

At first, different STxBs samples were analyzed with respect to the binding properties to $\mathrm{Gb}_{3}$. Afterwards, the phase preference of the different fluorescently labeled $\mathrm{Gb}_{3} \mathrm{~s}$ was investigated. Fluorescent properties of each fluorophore were measured with the fluorimeter. Then the phase behavior of the different labeled $\mathrm{Gb}_{3} \mathrm{~s}$ was analyzed, starting with the fatty acid labeled $\mathrm{Gb}_{3}$ s and ending with the study of the head group labeled $\mathrm{Gb}_{3} \mathrm{~s}$.

\subsection{Binding studies of $S T x B$ to its receptor lipid $G b_{3}$}

Three different STxB samples! ${ }^{1}$ were available to analyze the phase preference of $\mathrm{Gb}_{3}$ after $\mathrm{STxB}$ binding. The $\mathrm{STxB}$ binding to $\mathrm{Gb}_{3}$ and their purity was analyzed. The data from the STxB sample B were published in: Bosse M., Sibold J., Scheidt H. A., Patalag L. J., Kettelhoit K., Ries A., Werz D. B., Steinem C. \& Huster D. Shiga Toxin Binding Alters Lipid Packing and Domain Structure of $\mathrm{Gb}_{3}$-Containing

\footnotetext{
${ }^{1}$ The STxB samples A and C were purified by the group of Prof. Dr. Winfried Römer and B by the group of Prof. Dr. Daniel Huster (chapter 3.1.4)
} 
Membranes: A Solid-State NMR Study. Phys. Chem. Chem. Phys. 21, 1563015638 (2019).

The purity of three different STxB samples was analyzed with a $16 \%$ Schägger gel using two protein marker rows (Marker 1 ultra low range marker and Marker 2 PageRuler ${ }^{T M}$ ) and three different STxB preparations (figure 4.1). Both markers showed an exponential correlation from the molecular weight to the migration distance (seen as line, because the molecular weight is logarithmic plotted).

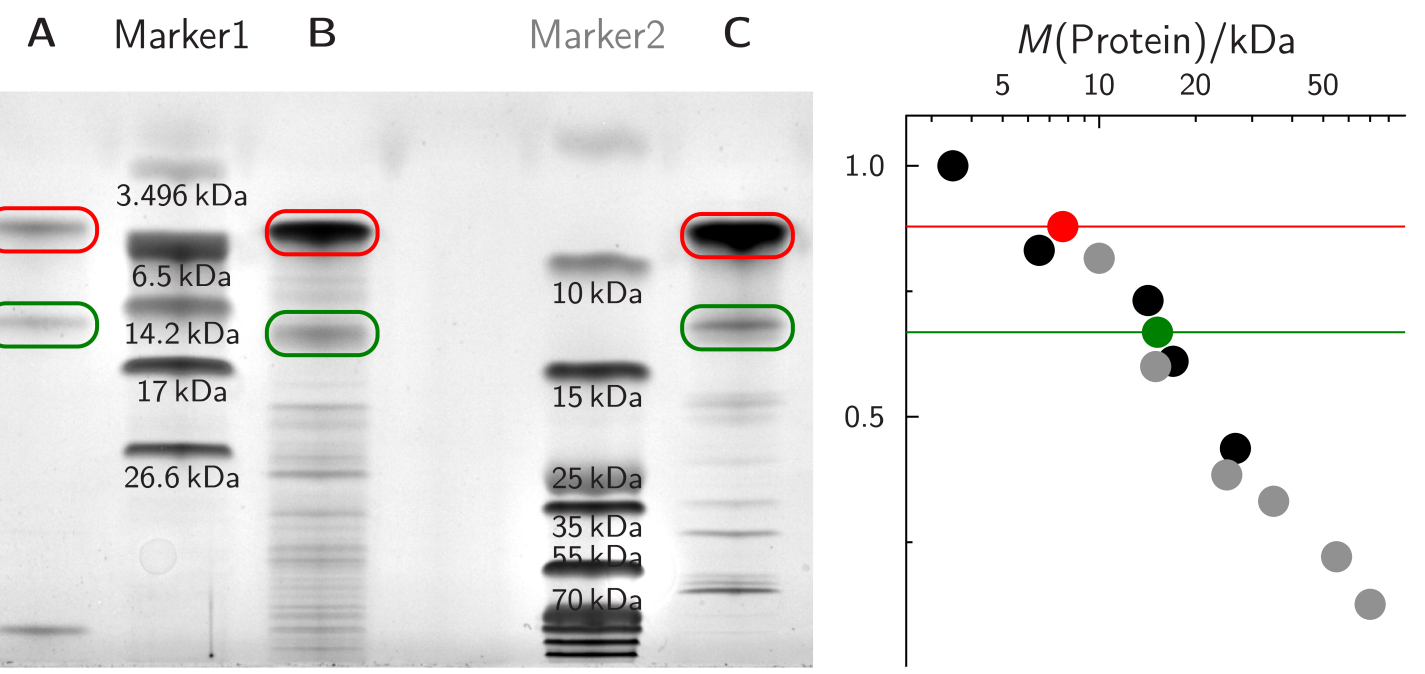

Figure 4.1.: A $16 \%$ Schägger gel with the three different B-subunits of shiga toxin (STxB) samples (A, B and C). To clarify the mass of the STxB two different protein markers were used: Marker 1 ultra low range marker and Marker 2 PageRuler ${ }^{T M}$. In red the protein bands of STxB are highlighted and in green the cluster of STxB. Beside the gel, the relative migration distances of the protein markers (Marker 1 black and Marker 2 grey) were plotted against the protein weights, showed in a logarithm scale. The two protein bands are also incorporated into the plot and the molecular weight of STxB (red dot) and the doubled weight (green dot) are marked.

The $16 \%$ Schägger gel shows that the protein bands with the highest intensities in all three samples (A, B and C) is marked in red (figure 4.1). The migration distance was plotted as red line and the calculated molecular weight of STxB $(7.7 \mathrm{kDa})[50$ as red dot in the relative distance weight plot (figure 4.1). The molecular weight of $\mathrm{STxB}$ and the migration distance were in good agreement to the protein markers. These bands were assigned to STxB. In all three STxB samples, a second protein band was visible marked in green (figure 4.1). The migration distance was plotted as a green line in the distance weight plot. The molecular weight of the STxB dimer $(15.4 \mathrm{kDa})$ was plotted as a green dot, which seemed to be in good agreement with the protein markers. However, the green marked bands were referred as STxB dimer. 
The different protein samples showed distinct amounts of impurities. The isolation A revealed one additional band with a molecular weight higher than $70 \mathrm{kDa}$. The STxB purification B showed several weak protein bands greater than $10 \mathrm{kDa}$ being presumably impurities. STxB isolation (C) has seven weak bands between 18 and $70 \mathrm{kDa}$.

The STxB binding to $\mathrm{Gb}_{3}$ was studied with adsorption isotherm measurements, performed by surface plasmon resonance (SPR) spectroscopy. A lipid monolayer composed of DOPC/porcine erythrocytes $\mathrm{Gb}_{3}$ ( $\mathrm{Gb}_{3 \text { porc }}$ ) (95:5) was spread on a hydrophobic (octan-1-thiol on gold (OT-Au)) functionalized SPR chip and the binding of different $\mathrm{STxB}$ concentrations were measured $(R$, detailed experiment set up in chapter 3.3.4). The data was normalized according to $300 \mathrm{~nm} \mathrm{STxB}\left(R_{\text {real }}\right)$ to allow a comparison of different experiments. The dissociation constant $\left(K_{\mathrm{d}}\right)$ is extracted by fitting the Langmuir adsorption isotherm

$$
R_{\text {real }}=\frac{R_{\text {real max }} \cdot c(\mathrm{STxB})}{c(\mathrm{STxB})+K_{\mathrm{d}}}
$$

to the data (figure 4.2). The concentration of $\operatorname{STxB}(c(\mathrm{STxB}))$ is the monomer concentration. The $K_{\mathrm{d}}$ values for the different STxB samples are listed in (table 4.1).

The $K_{\mathrm{d}}$ values range from 13 to $35 \mathrm{~nm}$ (figure 4.2 , table 4.1) which reflects a high binding affinity for all three STxB samples. In figure $4.2 \mathrm{D}$, the fits of all three STxB samples are compared. The STxB sample B has the highest affinity to the receptor lipid which is represented in the lowest $K_{\mathrm{d}}$ value of $12.9 \pm 3.2 \mathrm{~nm}$. The STxB sample $\mathrm{A}$ and $\mathrm{C}$ have $K_{\mathrm{d}}$ values of $17.4 \pm 2.5$ and $34.7 \pm 5.6 \mathrm{~nm}$ respectively. The binding affinity of the STxB samples A and B are comparable. The sample $\mathrm{C}$ showed a two times lower binding affinity to $\mathrm{Gb}_{3}$.

Table 4.1.: Dissociation constants $\left(K_{\mathrm{d}}\right)$ for the different STxB samples. The errors are the standard deviation calculated from the fit. The number of repeated adsorption isotherms are also listed.

\begin{tabular}{ccc} 
STxB sample & $K_{\mathrm{d}}[\mathrm{nm}]$ & number of scans \\
\hline A & $17.4 \pm 2.5$ & 4 \\
B & $12.9 \pm 3.2$ & 2 \\
C & $34.7 \pm 5.6$ & 3
\end{tabular}

$K_{\mathrm{d}}$ values for the STxB binding to cells are typically in the nM regime. 52 157 159 Schütte et al. measured $\mathrm{STxB} \mathrm{Gb}_{3}$ s binding affinities with a similar system using defined $\mathrm{Gb}_{3}$-species with different fatty acids in contrast to $\mathrm{Gb}_{3}$ mixture $\left(\mathrm{Gb}_{3 \text { porc }}\right)$ used in the experiments presented here. Their $K_{\mathrm{d}}$ values were in the range of 40 to 

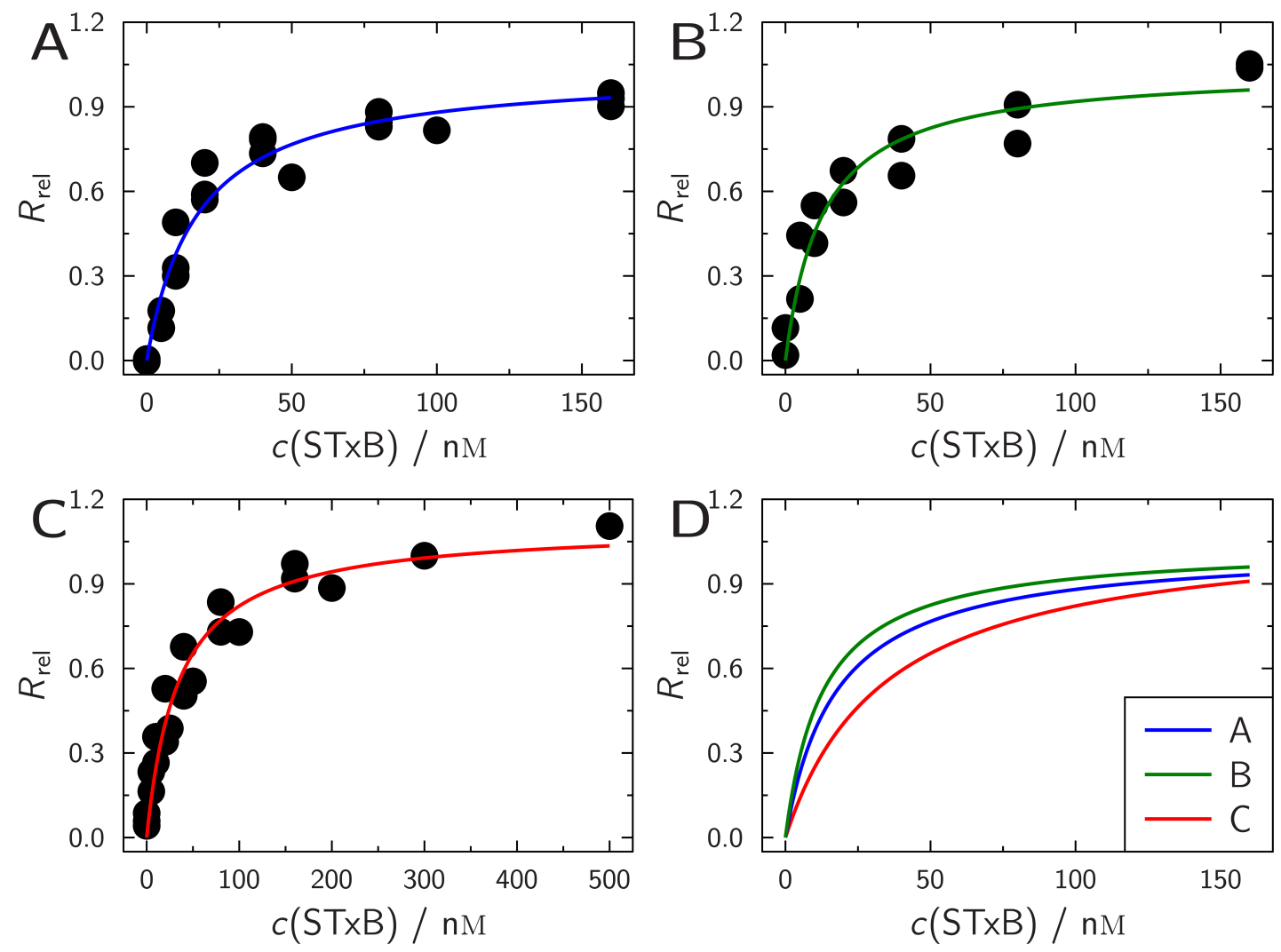

Figure 4.2.: Adsorption isotherm of three different B-subunit of shiga toxin (STxB) samples (A, B and C) bound to membranes composed of DOPC/Gb 3 porc (95:5). Langmuir adsorption isotherm (solid line) were fitted to the data and the dissociation constant is summarized in table 4.1. The fitted Langmuir adsorption isotherms are compared in D.

70 nM. 160 In an other binding study, performed by Head et al., the binding of STxB to $\mathrm{Gb}_{3}$ in microtiter wells was measured.

All three STxB samples show high binding affinities to $\mathrm{Gb}_{3}$, comparable to values found in literature. To enhance comparability, the STxB sample $\mathrm{C}$ was used in further experiments because this purification had the largest yield.

\subsection{Analysis of the phase preference of labeled $\mathrm{Gb}_{3} \mathrm{~s}$}

STxB binding was studied at cellular membranes as well as biomimetic model systems. $157 \mid 162$ The cellular membrane is very heterogeneous in terms of lipid composition and membrane structure. In the last two decades, nanoscaled domains differing in lipid composition with a higher order packing density have been discussed as functional units called lipid rafts. To mimic such domain structures, biomimetic models utilize lipid mixtures which phase separate in a $l_{\mathrm{o}}$, raft-like domains, and a liquid disordered $\left(l_{\mathrm{d}}\right)$ phase. In studies using GUVs, the $\mathrm{Gb}_{3} \mathrm{~s}$ showed preferential 
binding of STxB to the $l_{\mathrm{o}}$ phase in lipid mixture consisting of DOPC/sphingomyelin (SM)/cholesterol (Chol)/Gb3porc $(40: 35: 20: 5) .54$ From this observation, it can be concluded that $\mathrm{Gb}_{3}$ is enriched in the $l_{\mathrm{o}}$ phase after binding. $160 \mid 162$ However, it is not clear, where $\mathrm{Gb}_{3}$ is located in phase-separated membranes before STxB binding. To get information about the phase distribution of $\mathrm{Gb}_{3}$ before $\mathrm{STxB}$ binding, fluorescently labeled $\mathrm{Gb}_{3} \mathrm{~s}$ were synthesized in the group of Prof. Dr. Daniel B. Werz (TU Braunschweig, Institut für Organische Chemie, Braunschweig (Germany), scheme A.1. Their phase preference was investigated in phase-separated GUVs. One solution was the design of fluorescently labeled fatty acids with pentaene or hexaene moieties which are connected to the $\mathrm{Gb}_{3}$ (figure 3.11). These results are published in: Patalag L. J., Sibold J., Schütte O. M., Steinem C. \& Werz D. B. Gb 3 Glycosphingolipids with Fluorescent Oligoene Fatty Acids: Synthesis and Phase Behavior in Model Membranes. ChemBioChem 18, 2171-2178 (2017). The second solution was to connect the fluorophore to the second sugar of the $\mathrm{Gb}_{3}$ with a polyethylene glycol (PEG) linker in between (figure 3.12). These results are submitted as: Sibold J., Kettelhoit K., Vuong L., Liu F., Werz D. B. \& Steinem C. Synthesis of Head Group Labeled Gb3 Glycosphingolipids and Their Distribution in Phase-Separated Giant Unilamellar Vesicles. Angew. Chem. Int. Ed. 58, 17805-17813 (2019). In this study, the fluorescent properties of the $\mathrm{Gb}_{3} \mathrm{~s}$ derivates were determined as well as their preferential phase distribution in biomimetic membranes.

\subsubsection{Fluorescent properties of labeled $G b_{3} s$}

The fluorescently labeled $\mathrm{Gb}_{3} \mathrm{~s}$ used in this work are divided in two categories, fatty acid labeled $\mathrm{Gb}_{3} \mathrm{~s}$ and head group labeled $\mathrm{Gb}_{3} \mathrm{~s}$ (scheme A.1). Two different fatty acid labeled $\mathrm{Gb}_{3} \mathrm{~s}$, the $\mathrm{Gb}_{3}$ phenyl-modified fatty acid $\left(\mathrm{Gb}_{3} \mathrm{PH}\right)$ and $\mathrm{Gb}_{3}$ thienothienylmodified fatty acid $\left(\mathrm{Gb}_{3} \mathrm{TT}\right)$ (scheme 3.11), were analyzed. Additionally, eight different head group labeled $\mathrm{Gb}_{3}$ s were used (scheme 3.12). All the eight different head group labeled $\mathrm{Gb}_{3} \mathrm{~s}$ had a BODIPY as fluorophore and $\mathrm{Gb}_{3} \mathrm{PEG}_{13} \mathrm{C}_{24: 0} \mathrm{H}$ was exemplary analyzed. Absorption and emission spectra were measured by fluorimetry to determine the fluorescent properties of the $\mathrm{Gb}_{3} \mathrm{~s}$. Therefore, GUVs composed of DOPC/Gb 3 (99:1) were prepared for all $\mathrm{Gb}_{3}$ derivates. The amount of lipids in the analyzing solution was $16 \mu \mathrm{M}$, determined by the phosphor concentration of the GUV solution.

The excitation spectra of the fatty acid labeled $\mathrm{Gb}_{3}$ s were measured at an emission wavelength of $465 \mathrm{~nm}$ (figure $4.3 \mathrm{~A}$ and $\mathrm{B}$, red line). $\mathrm{Gb}_{3} \mathrm{PH}$ showed two maxima at

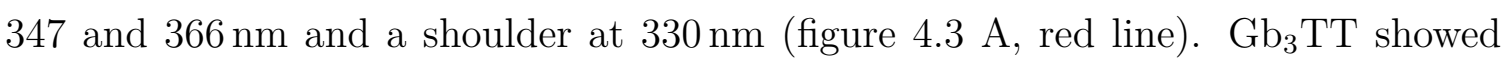


two peaks at 369 and $391 \mathrm{~nm}$ (figure $4.3 \mathrm{~B}$ red line). The excitation spectra of the head group labeled $\mathrm{Gb}_{3}$ were measured at the emission wavelength of $580 \mathrm{~nm}$. The excitation range of BODIPY is from 460 to $520 \mathrm{~nm}$ with a maximum at $506 \mathrm{~nm}$ (figure 4.3 C red line).
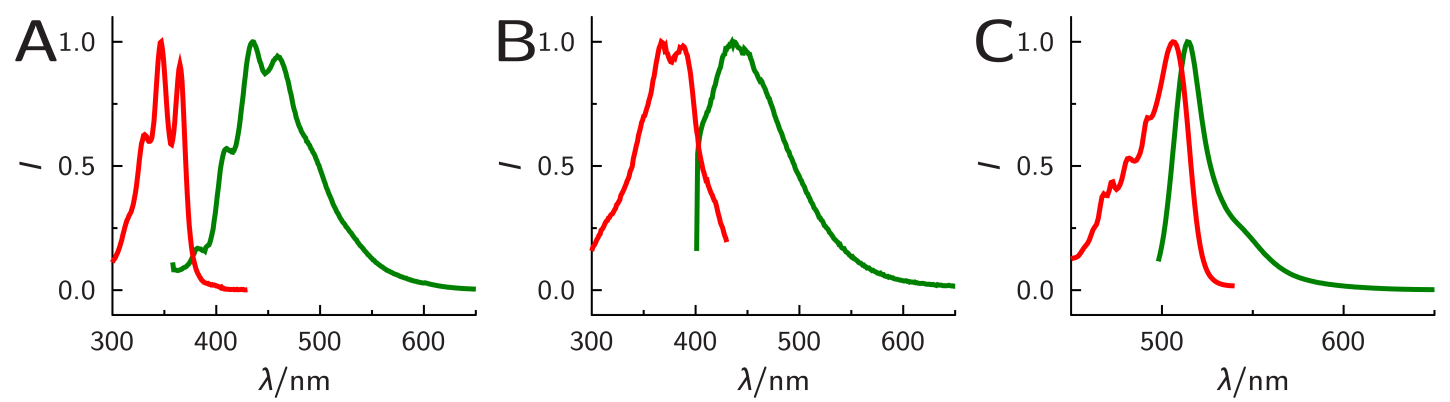

Figure 4.3.: Excitation (red) and emission (green) spectra of labeled globotriaosyl ceramides $\left(\mathrm{Gb}_{3} \mathrm{~s}\right)$ in GUVs composed of $\mathrm{DOPC} / \mathrm{Gb}_{3}$ (99:1). The lipid concentration in the cuvette was set to $16 \mu \mathrm{M}$. The fluorescence excitation spectra were measured from $\lambda=300$ to $430 \mathrm{~nm}$, with $\lambda_{\mathrm{em}}=465 \mathrm{~nm}$ for $\mathrm{Gb}_{3} \mathrm{PH}(\mathrm{A})$ and $\mathrm{Gb}_{3} \mathrm{TT}(\mathrm{B})$ and from $\lambda=450$ to $540 \mathrm{~nm}$, with $\lambda_{\mathrm{em}}=580 \mathrm{~nm}$ for $\mathrm{Gb}_{3} \mathrm{PEG}_{13} \mathrm{C}_{24: 0} \mathrm{H}(\mathrm{C})$. Fluorescence emission was excited at $\mathrm{A}$ ) $\lambda_{\text {ex }}=348 \mathrm{~nm}$, recorded from $\lambda=360$ to $650 \mathrm{~nm}$, B) $\lambda_{\text {ex }}=391 \mathrm{~nm}$, recorded from $\lambda=400$ to $650 \mathrm{~nm}$ and C) $\lambda_{\mathrm{ex}}=488 \mathrm{~nm}$, recorded from $\lambda=500$ to $650 \mathrm{~nm}$.

The emission spectrum of $\mathrm{Gb}_{3} \mathrm{PH}$ was measured with excitation at $348 \mathrm{~nm}$, resulting in a broad range from 400 to $600 \mathrm{~nm}$ with two peaks at 434 and $458 \mathrm{~nm}$ and a shoulder at $410 \mathrm{~nm}$ (figure 4.3 A green line). The intensity maximum is at $434 \mathrm{~nm}$. The excitation wavelength for the emission spectrum $\mathrm{Gb}_{3} \mathrm{TT}$ was $391 \mathrm{~nm}$. The emission of $\mathrm{Gb}_{3} \mathrm{TT}$ showed a broad range from 400 to $600 \mathrm{~nm}$, with a maximum around $450 \mathrm{~nm}$ (figure $4.3 \mathrm{~B}$ green line). The exemplarily measured head group labeled $\mathrm{Gb}_{3}$ was excited at $488 \mathrm{~nm}$ and showed a peak from 500 to $570 \mathrm{~nm}$ with a maximum at $514 \mathrm{~nm}$ (figure 4.3 C green line).

The excitation spectra of the fatty acid labeled $\mathrm{Gb}_{3}$ s show typical bands for the vibrational resolution of polyene chromophores. $\frac{163}{163}$ The excitation spectra as well as the emission spectra of the fatty acid labeled $\mathrm{Gb}_{3} \mathrm{~s}$ are red shifted compared to the excitation and emission spectra of sphingosine-like chain containing a pentaene 164 which is a result of the addition from the ring structures. The red shift of the $\mathrm{Gb}_{3} \mathrm{TT}$ compared to $\mathrm{Gb}_{3} \mathrm{PH}$ is a result of the thiophene group instead of the phenyl ring.

The excitation and emission spectra of the BODIPY in the head group labeled $\mathrm{Gb}_{3} \mathrm{~S}$ is similar to other BODIPY labeled lipids found elsewhere. $165+172$

To measure the phase distribution of the labeled $\mathrm{Gb}_{3} \mathrm{~s}$, the different phases in the phase-separated GUVs were marked. There are many different phase markers and the best phase markers exist for the $l_{\mathrm{d}}$ phase. $139 \mid 140$ 
The aim of this work is to quantitatively measure the distribution of $\mathrm{Gb}_{3}$ in the lipid phases and STxB binding by fluorescence microscopy. Quantitative analysis for the phase preference of the $\mathrm{Gb}_{3} \mathrm{~s}$ can only be obtained from fluorescence images if no significant fluorescence resonance energy transfer (FRET) between the labeled $\mathrm{Gb}_{3} \mathrm{~S}$ and the $l_{\mathrm{d}}$ marker exists. This ensures that the measured fluorescence intensities fully reassemble the $\mathrm{Gb}_{3}$ distribution. A FRET effect is detectable in the emission spectrum as an emission of the acceptor fluorophore $\left(l_{\mathrm{d}}\right.$ marker) by excitation of the donor fluorophore $\left(\mathrm{Gb}_{3} \mathrm{~s}\right)$. This is possible if the emission spectrum of the donor fluorophore overlaps with the excitation spectrum of the acceptor fluorophore. To control this, the donor fluorophore was excited and the emission spectrum of the GUVs which contains the two fluorophores was measured.

The two different $l_{\mathrm{d}}$ phase marker, TexasRed-DHPE and Dy731-DOPE, were analyzed regarding possible FRET effects with the fatty acid labeled $\mathrm{Gb}_{3} \mathrm{~s}$ and the BODIPY used for the head group labeled derivates. The analysis was performed in GUVs composed of DOPC/Gb $/ l_{\mathrm{d}}$ marker (98:1:1). The lipid concentration in the cuvette was $16 \mu \mathrm{M}$, determined by the phosphor concentration of the GUV solution. The emission spectra of the six different GUV solutions were measured by means of fluorimeter.

The excitation wavelengths for the emission spectra are 348, 391 and $488 \mathrm{~nm}$ for $\mathrm{Gb}_{3} \mathrm{PH}, \mathrm{Gb}_{3} \mathrm{TT}$ and $\mathrm{Gb}_{3} \mathrm{PEG}_{13} \mathrm{C}_{24: 0} \mathrm{H}$, respectively (figure 4.4). The emission spectra of the three labeled $\mathrm{Gb}_{3} \mathrm{~s}$ without a $l_{\mathrm{d}}$ phase marker were plotted as reference in the diagrams (figure 4.4 green). In blue, the emission spectra of the $\mathrm{Gb}_{3} \mathrm{~s}$ and TexasRedDHPE are shown. All three $\mathrm{Gb}_{3}$ s showed a FRET with TexasRed-DHPE at $610 \mathrm{~nm}$. The efficiency of the FRET is higher than $50 \%$ for all labeled $\mathrm{Gb}_{3}$-species. The FRET effect of the labeled $\mathrm{Gb}_{3} \mathrm{~s}$ and the second $l_{\mathrm{d}}$ marker, Dy731-DOPE, is shown in red in figure 4.4. These emission spectra overlapped nicely with the emission spectra without a $l_{\mathrm{d}}$ marker. As the emission of Dy731-DOPE at $760 \mathrm{~nm}$ is very low, this part of the emission spectrum is zoomed in (upper right corner figure 4.4). The FRET efficiency was determined as $<3 \%$ (figure $4.4 \mathrm{~A}, \mathrm{~B}$ ) for the fatty acid labeled $\mathrm{Gb}_{3} \mathrm{~S}$ and $<0.5 \%$ for the head group labeled $\mathrm{Gb}_{3}$ (figure $4.4 \mathrm{C}$ ). These effects are very low and have a minor influence for the determination of quantitative information from fluorescence signal of the $\mathrm{Gb}_{3} \mathrm{~s}$.

Other effects which might influence the quantitative evaluation of the fluorescence from $\mathrm{Gb}_{3}$ are possible quenching effects from salts or even self-quenching of the fluorophores. Quenching reduces the measured intensity of the fluorophores and therefore, it would lead to errors when determining quantitative information from fluorescence images. The self-quenching effect depends on the distance between two fluo- 


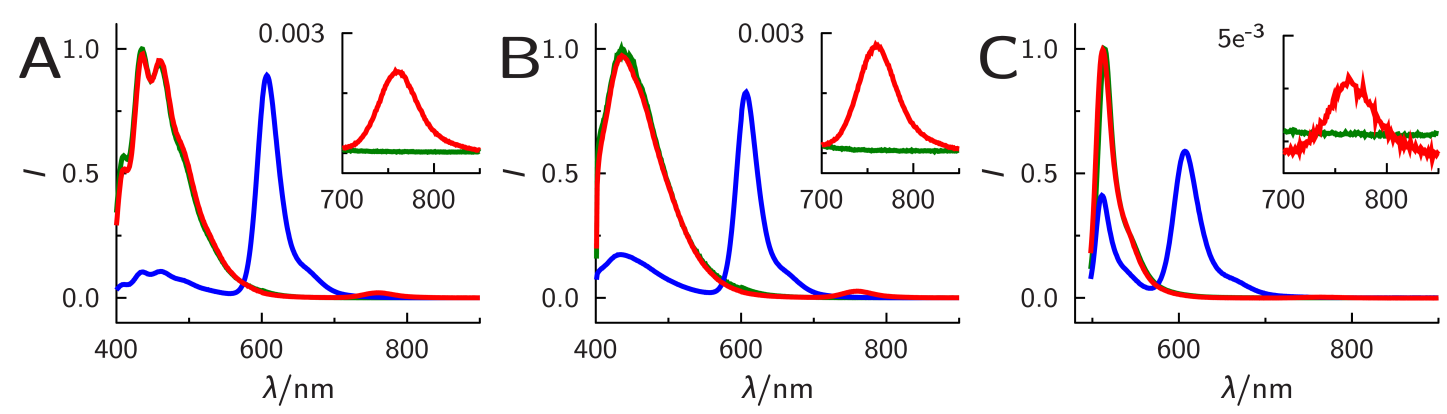

Figure 4.4.: Fluorescence spectra of A) $\left.\mathrm{Gb}_{3} \mathrm{PH}\left(\lambda_{\mathrm{ex}}=348 \mathrm{~nm}\right), \mathrm{B}\right) \mathrm{Gb}_{3} \mathrm{TT}\left(\lambda_{\mathrm{ex}}=391 \mathrm{~nm}\right)$ and $\mathrm{C}) \mathrm{Gb}_{3} \mathrm{PEG}_{13} \mathrm{C}_{24: 0} \mathrm{H}\left(\lambda_{\mathrm{ex}}=488 \mathrm{~nm}\right)$. Green lines: fluorescence spectra obtained from GUVs composed of DOPC/Gb 3 (99:1); blue lines: fluorescence spectra obtained from GUVs composed of DOPC/Gb $/$ /TexasRed-DHPE (98:1:1); red lines: fluorescence spectra obtained from GUVs composed of DOPC/Gb $3 /$ Dy731-DOPE (98:1:1). The lipid concentration in the cuvette was set to $16 \mu \mathrm{M}$.

rophore molecules and therefore, it is directly connected to the fluorophore concentration in the lipid mixture. GUVs with varying fluorophore concentration (DOPC/ $\left.\mathrm{Gb}_{3}(1-\mathrm{x}: \mathrm{x})\right)$ were prepared and studied using fluorimetry. The measured excitation intensity of the GUV solution (F) was compared with the emission intensity of the same solution after lysis of the GUVs using Triton X-100 (1 mM, lipid solution, $\left.\mathrm{F}_{0}\right)$. Lysis leads to a homogeneous distribution of the lipid material in the solution and fluorophore-fluorophore distance is drastically increased.173 To make sure that the distance in the lipid solution is large enough, a low lipid concentration of $16 \mu \mathrm{M}$ in the cuvette was used for the self-quenching experiments (figure 4.5).
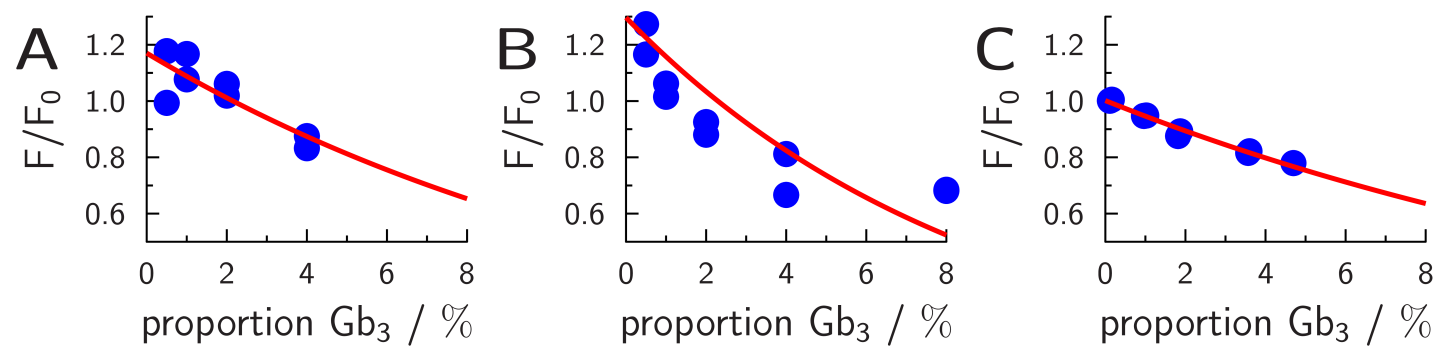

Figure 4.5.: Self-quenching effect of labeled globotriaosyl ceramide $\left(\mathrm{Gb}_{3}\right)$. The fluorescence intensity of GUVs (DOPC/Gb 3 (1-x:x)) was recorded before (F) and after Triton $\mathrm{X}-100$ addition $\left(\mathrm{F}_{0}\right)$. The excitation wavelengths of the different $\mathrm{Gb}_{3}$ s were 348, 391 and $488 \mathrm{~nm}$ for $\mathrm{Gb}_{3} \mathrm{PH}(\mathrm{A}), \mathrm{Gb}_{3} \mathrm{TT}(\mathrm{B})$ and $\mathrm{Gb}_{3} \mathrm{PEG}_{13} \mathrm{C}_{24: 0} \mathrm{H}(\mathrm{C})$, respectively. The $\mathrm{Gb}_{3}$ concentration in the GUVs of $\mathrm{Gb}_{3} \mathrm{PEG}_{13} \mathrm{C}_{24: 0} \mathrm{H}$ was measured with adsorptions spectra.

In figure 4.5, the self-quenching effects of $\mathrm{Gb}_{3} \mathrm{~s}$ labeled with three different fluorophores are shown. The normalized fluorescence intensity $\left(\mathrm{F} / \mathrm{F}_{0}\right)$ decreases with increasing labeled $\mathrm{Gb}_{3}$ proportion in the GUVs showing, a self-quenching effect. The 
self-quenching effect theoretically shows an exponential course that was adjusted (red line). 173

To allow comparison of different experiments, the absolute concentration of $\mathrm{Gb}_{3}$ in the cuvette is important to be known and is typically measured by absorption measurements (UV/Vis). Because the absorbance of the fatty acid labeled $\mathrm{Gb}_{3} \mathrm{~s}$ is very weak, the overall signal intensity was too low to allow the determination of $\mathrm{Gb}_{3}$ concentration in the GUVs by absorption measurements. Instead, the concentrations in the GUVs were approximated by the amount of the fatty acid labeled $\mathrm{Gb}_{3} \mathrm{~s}$ used for the preparation of the GUVs.

For low $\mathrm{Gb}_{3}$ concentrations, the relative fluorescence intensity of the fatty acid labeled $\mathrm{Gb}_{3}$ is above one (figure $4.5 \mathrm{~A}$ and $\mathrm{B}$ ), indicating either a dequenching effect of the fluorophores or a quenching effect of Triton X-100 to the fatty acid labeled $\mathrm{Gb}_{3} \mathrm{~s}$. The used concentrations are in a regime where self-quenching does not play a role, 173 making an interaction between Triton X-100 and fatty acid labeled $\mathrm{Gb}_{3}$ more probable.

The determination of the concentration of head group labeled $\mathrm{Gb}_{3}$ in $\mathrm{GUVs}$ was performed by UV/Vis. The self-quenching measurement of $\mathrm{Gb}_{3} \mathrm{PEG}_{13} \mathrm{C}_{24: 0} \mathrm{H}$, as an example of the head group labeled $\mathrm{Gb}_{3} \mathrm{~s}$, is shown in figure 4.5 C. The relative fluorescence intensity of the BODIPY head group labeled $\mathrm{Gb}_{3}$ were not above one, proving that no quenching effects of Triton X-100 to the fluorophore exist.

To avoid self-quenching effects of the fluorophore within the measurements head group labeled $\mathrm{Gb}_{3}$ was used in low amounts of $1 \mathrm{~mol} \%$ of $\mathrm{Gb}_{3}$ to ensure no artifacts were present in the calculation of the phase distribution of the $\mathrm{Gb}_{3}$ species.

Measurements at $1 \mathrm{~mol} \%$ fatty acid labeled $\mathrm{Gb}_{3}$ in GUVs were not executable, due to the very low signal-to-noise ratio resulting from the low absorption properties. Therefore, concentration of these $\mathrm{Gb}_{3}$ species was increased to $5 \mathrm{~mol} \%$, allowing to detect phase distributions of the fatty acid labeled $\mathrm{Gb}_{3} \mathrm{~s}$. As shown in figure $4.5 \mathrm{~A}$ and $\mathrm{B}$, this is a compromise as it represents the lowest possible concentration considering the signal detection, but measurements are influenced by self-quenching effects.

For the quantitative measurements, the measurement parameter for the following phase distribution of $\mathrm{Gb}_{3}$ studies was chosen: The fatty acid labeled $\mathrm{Gb}_{3} \mathrm{~S}$ was incorporated in phase-separated GUVs with $5 \mathrm{~mol} \%$ and the head group labeled $\mathrm{Gb}_{3}$, with the better signal to noise ratio, with $1 \mathrm{~mol} \%$. 


\subsubsection{Preference of fatty acid labeled $\mathrm{Gb}_{3} \mathrm{~s}$ in phase-separated GUVs}

The phase preference of the two fatty acid labeled $\mathrm{Gb}_{3} \mathrm{~s}\left(\mathrm{~Gb}_{3} \mathrm{PH}\right.$ and $\mathrm{Gb}_{3} \mathrm{TT}$, scheme 3.11 was analyzed in model membrane systems. The $\mathrm{Gb}_{3} \mathrm{~s}$ were included in

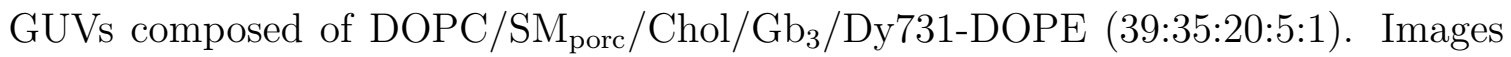
of GUVs were recorded along the Z-axis for both fluorophores, the fatty acid labeled $\mathrm{Gb}_{3}$ and the $l_{\mathrm{d}}$ marker, Dy731-DOPE. From each slide of these Z-stacks, two profile lines were analyzed to calculate the $l_{\mathrm{o}}$ phase distribution $\left(\% l_{\mathrm{o}}\right)$ as described in section 3.4.1 (figure 3.2). The results from the different line scans were shown as violin plots for each of the $\mathrm{Gb}_{3}$-species (figure 4.6).

As shown in figure 4.6. $\mathrm{Gb}_{3} \mathrm{PH}$ is nearly homogeneously distributed in the $l_{\mathrm{o}} / l_{\mathrm{d}}$ phase-separated membrane with a $\% l_{\mathrm{o}}$ of 0.45 . On the other hand, nearly one quarter of the $\mathrm{Gb}_{3} \mathrm{TT}$ is present in the $l_{\mathrm{o}}$ phase $\left(\% l_{\mathrm{o}}=0.24\right.$, figure 4.6 , table 4.2 . The distribution width of the two fatty acid labeled $\mathrm{Gb}_{3} \mathrm{~s}$ differs by a factor of 2 . The standard deviation of $\mathrm{Gb}_{3} \mathrm{PH}$ is with 0.22 double as high as for $\mathrm{Gb}_{3} \mathrm{TT}$, this can be an artefact of the self-quenshing from the fluorophores.

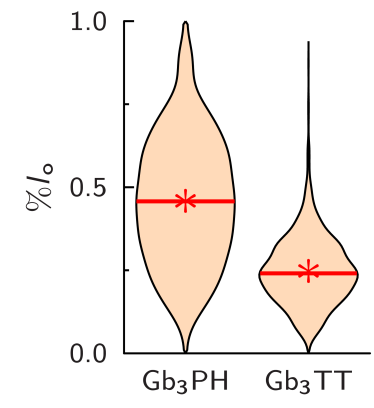

Figure 4.6.: Liquid ordered phase distribution $\left(\% l_{0}\right)$ of the different fatty acid labeled globotriaosyl ceramides $\left(\mathrm{Gb}_{3} \mathrm{~s}\right)$ in GUVs composed of DOPC/SM $\mathrm{Sorc}_{\text {c }} / \mathrm{Chol} / \mathrm{Gb}_{3} / \mathrm{Dy} 731-\mathrm{DOPE}$ (39:35:20: $5: 1)$ visualized as violin plots. The mean values are given as stars and the medians are represented with a stroke. The number of profile lines as well as the mean and the standard deviation are listed in table 4.2 .

The fatty acid labeled $\mathrm{Gb}_{3} \mathrm{~s}$ are preferentially located in the $l_{\mathrm{d}}$ phase, in contrast to native $\mathrm{Gb}_{3} \mathrm{~s}$ which are found in the detergent restistant membranes (DRMs) from primary human blood microvascular endothelial cells (pHBMEC). 47 A DRM extraction was done by Legros et al. to distinguish between the raft domains (DRM fraction, mimic as $l_{\mathrm{o}}$ phase) and fluid membrane (non-DRM fraction, mimic as $l_{\mathrm{d}}$ phase). 174.176 It has been shown, that the modification of sphingolipids with bulky fatty acids (as BODIPY or NBD) changes the phase preference of the lipid from $l_{\mathrm{o}}$ phase to the $l_{\mathrm{d}}$ phase. $139|142| 177 \mid 178$ The bulky element in the fatty acid is excluded from the $l_{\mathrm{o}}$ phase,

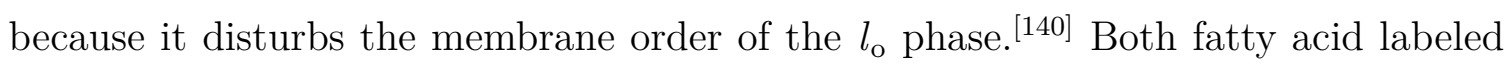
$\mathrm{Gb}_{3} \mathrm{~s}$ have a bulky structures in the modified chains (scheme 3.11). This might be the reason for the $l_{\mathrm{d}}$ phase preference. This explanation is supported by the findings, that the $\mathrm{Gb}_{3} \mathrm{TT}$ is more soluble in the $l_{\mathrm{d}}$ phase as $\mathrm{Gb}_{3} \mathrm{PH}\left(\% l_{\mathrm{o}}=0.24 \pm 0.10\right.$ 
to $0.45 \pm 0.22$ ). The fatty acid of $\mathrm{Gb}_{3} \mathrm{PH}$ has a phenyl group and a long fatty acid. The fatty acid of $\mathrm{Gb}_{3} \mathrm{TT}$ has a thienothienyl ring system which are two rings and a branched chain end which also increases the sterics of the fatty acid.

Table 4.2.: Mean values of the liquid ordered phase distribution $\left(\% l_{\mathrm{o}}\right)$ for the different fatty acid labeled $\mathrm{Gb}_{3} \mathrm{~s}$ without (-) and with (+) B-subunit of shiga toxin (STxB) (300 nM). The data are shown as $\mathrm{m} \pm \mathrm{sd}(N)$; m: mean value, sd: standard deviation, $N$ : numbers of analyzed profile lines.

\begin{tabular}{c|cc}
$\% l_{\mathrm{o}}(N)$ & $\mathrm{Gb}_{3} \mathrm{PH}$ & $\mathrm{Gb}_{3} \mathrm{TT}$ \\
\hline -STxB & $0.45 \pm 0.22(2175)$ & $0.24 \pm 0.10(2003)$ \\
$+\mathrm{STxB}$ & $0.41 \pm 0.21(2103)$ & $0.24 \pm 0.11(2329)$
\end{tabular}

Upon binding of STxB, a reorganization within the lipid membrane might be happening and the preferred phase of the $\mathrm{Gb}_{3} \mathrm{~s}$ might change to the $l_{\mathrm{o}}$ phase because the STxB binds the natural $\mathrm{Gb}_{3}$ in the $l_{\text {o phase. } 54|160| 162}$ To study this, GUVs were analyzed in the same way as described before, in presence of $300 \mathrm{~nm}$ STxB in the GUV solution. These $\mathrm{Gb}_{3}$ distributions were compared with GUVs which were not incubated with STxB (figure 4.7, table 4.2). The violin plots of the $\% l_{\mathrm{o}}$ of the $\mathrm{Gb}_{3}$ without STxB (-STxB) looks similar to the violin plots with STxB for both fatty acid labeled $\mathrm{Gb}_{3} \mathrm{~s}$. The distribution of both fatty acid labeled $\mathrm{Gb}_{3} \mathrm{~s}$ did not change significantly (figure 4.7, table 4.2 and A.4.
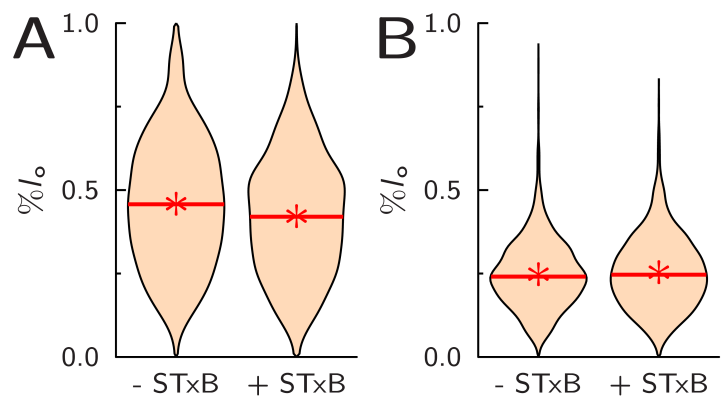

Figure 4.7.: Violin plots of liquid ordered phase distribution $\left(\% l_{\mathrm{o}}\right)$ from different fatty acid labeled $\mathrm{Gb}_{3} \mathrm{~s}$ before (-STxB) and after binding of $\mathrm{STxB}(+\mathrm{STxB}, 300 \mathrm{nM})$. The measurement was done with GUVs composed of DOPC/SM porc $/ \mathrm{Chol} / \mathrm{Gb}_{3} /$ Dy731-DOPE (39:35:20:5:1). In $\mathrm{A}$, the $\% l_{\mathrm{o}}$ of $\mathrm{Gb}_{3} \mathrm{PH}$ and in $\mathrm{B}$, the $\% l_{\mathrm{o}}$ of $\mathrm{Gb}_{3} \mathrm{TT}$ are compared to the binding of STxB. The mean values are given as stars and the medians are represented with a stroke. The number of analyzed profile lines as well as the mean and the standard deviation is listed in table 4.2 .

The binding of STxB to phase-separated GUVs with fatty acid labeled $\mathrm{Gb}_{3} \mathrm{~s}$ did not change the phase distribution of the $\mathrm{Gb}_{3} \mathrm{~s}$. There are two possible interpretations for this result: First, the STxB did not bind to the $\mathrm{Gb}_{3} \mathrm{~s}$ and second, the STxB 
binds to the $l_{\mathrm{d}}$ phase and the binding did not shift the phase distribution of the fatty acid labeled $\mathrm{Gb}_{3} \mathrm{~s}$. To check the binding of STxB to the $l_{\mathrm{d}}$ phase, the phase-separated GUVs with the fatty acid labeled $\mathrm{Gb}_{3}$ s were incubated with $300 \mathrm{~nm}$ Cy3 labeled STxB (figure 4.8). The intensity images and the radial profile of STxB is co-localized with the intensity of Dy731-DOPE (figure 4.8). This observation suggests that the binding of $\mathrm{STxB}$ to fatty acid labeled $\mathrm{Gb}_{3}$ is located in the $l_{\mathrm{d}}$ phase.
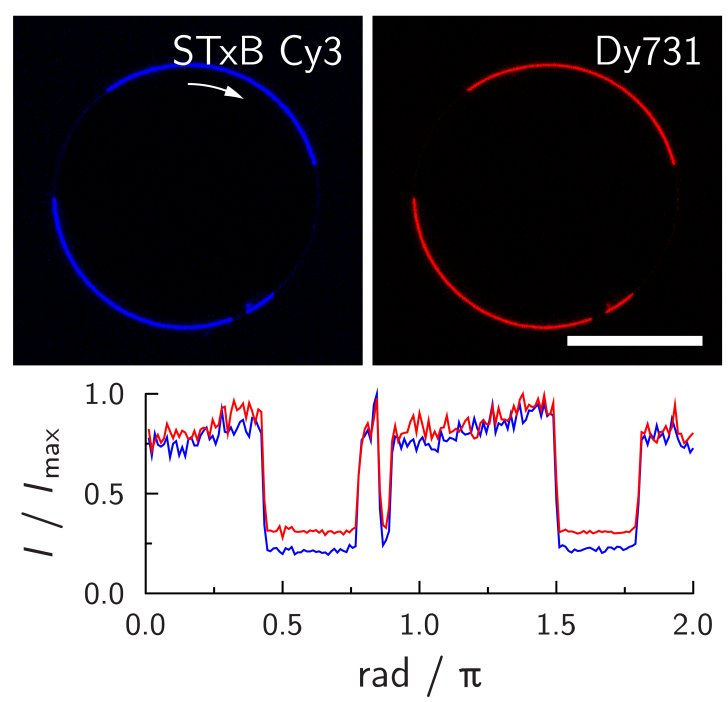
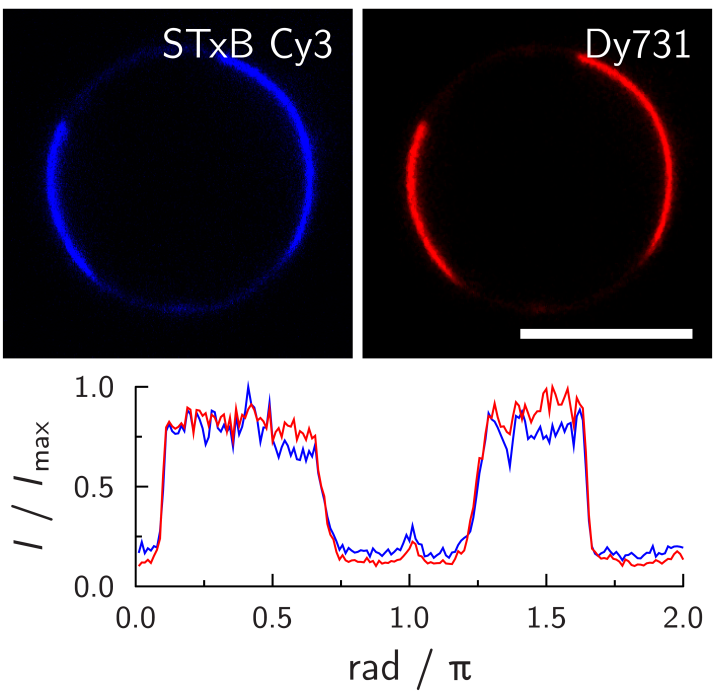

Figure 4.8.: Confocal fluorescence image of phase-separated GUVs composed of DOPC/ $\mathrm{SM}_{\text {porc }} / \mathrm{Chol} / \mathrm{Gb}_{3} / \mathrm{Dy} 731-\mathrm{DOPE}$ (39:35:20:5:1) with $300 \mathrm{~nm}$ Cy3 labeled STxB in solution. The fluorescence image from the $\mathrm{STxB}$ is shown in blue and from the phase marker Dy731DOPE is shown in red. Underneath the circular profile of the GUV intensity of both fluorophores is plotted, starting at twelve o'clock and going clockwise. A) represents a

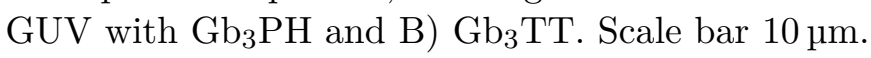

Using natural $\left[54[55]\right.$ and synthetic unlabeled $\mathrm{Gb}_{3} \mathrm{~s}, \underline{160 \mid 162} \mathrm{STxB}$ binds to the $l_{\mathrm{o}}$ phase which is not in agreement with the fatty acid labeled $\mathrm{Gb}_{3} \mathrm{~s}$. A similar phenomena was reported in studies with B-subunit of cholera toxin (CTxB) which binds to its fatty acid labeled receptor pentasaccharide ganglioside $\left(\mathrm{GM}_{1}\right) .142|177| 179 \mid 180$. While CTxB is known to bind to $\mathrm{GM}_{1}$ in the $l_{\mathrm{o}}$ phase, the fatty acid labeled $\mathrm{GM}_{1}$ binding to $\mathrm{CTxB}$ was observed in the $l_{\mathrm{d}}$ phase. $142|181| 182$ The change in the fatty acid from an alkyl to a bulky ring system changed the packing density of $\mathrm{Gb}_{3}$. The phase preference of the receptor is the $l_{\mathrm{d}}$ phase and therefore protein binding also takes place in the $l_{\mathrm{d}}$ phase. From this observation, it can be concluded that the phase preference and distribution of the fatty acid labeled $\mathrm{Gb}_{3} \mathrm{~s}$ does not represent the phase preference and distribution of the natural $\mathrm{Gb}_{3} \mathrm{~s}$. 


\subsubsection{Preference of head group labeled $\mathrm{Gb}_{3} \mathrm{~s}$ in phase-separated GUVs}

The fatty acid labeled $\mathrm{Gb}_{3}$ derivatives are preferentially distributed in the $l_{\mathrm{d}}$ phase and bind $\mathrm{STxB}$ in the $l_{\mathrm{d}}$ phase in contrast to natural $\mathrm{Gb}_{3} \mathrm{~S}$ which are typically bound by $\mathrm{STxB}$ in the $l_{\mathrm{o}}$ phase.5455 To mimic the natural situation head group labeled derivatives were synthesized. The head group of $\mathrm{Gb}_{3}$ consists of a trisaccharide which is only partially involved in the binding of STxB to $\mathrm{Gb}_{3} \cdot \frac{50|183| 184}{1}$ The 2'hydroxyl-group of the $\beta$-galactose of the $\mathrm{Gb}_{3}$ head group does not contribute to the process of STxB binding as shown by crystal structure analysis 50 and binding studies of trisaccarides. $183 \mid 184$ To ensure the head group labeled derivatives mimic the situation found in cellular systems in terms of phase preference and STxB binding, the uninvolved hydroxyl group was used to connect the fluorophore BODIPY via a PEG linker to the $\mathrm{Gb}_{3}$ (scheme 3.12). Unlike natural $\mathrm{Gb}_{3}$ which are a mixture of 16 to 24 carbon atoms in the alkyl chains, the fatty acid of the head group labeled $\mathrm{Gb}_{3} \mathrm{~S}$ is based on the $\mathrm{C}_{24}$ fatty acid. $\mathrm{C}_{24}$ was chosen, because they are a major part of the $\mathrm{Gb}_{3} \mathrm{~s}$ in erythrocytes, 185 HeLa cells $\frac{186}{186}$ and HEp-2 cells. 187. In addition, natural $\mathrm{Gb}_{3} \mathrm{~s}$ show $\alpha$-hydroxylation. The group of Prof. Dr. Daniel B. Werz synthesized head group labeled $\mathrm{Gb}_{3} \mathrm{~s}$ with different fatty acids to enable the investigation of the influence of the fatty acid saturation and hydroxylation on the phase distribution. In total, eight different head group labeled $\mathrm{Gb}_{3} \mathrm{~s}$, with three chemical modifications were synthesized. To encode the information of the chemical structure of the $\mathrm{Gb}_{3}$ the following abbreviation is used: $\mathrm{Gb}_{3} \mathrm{PEG}_{\mathrm{n}} \mathrm{C}_{24: \Delta} \mathrm{X}$ (scheme 3.12). The linker length of the PEG linker (n) was chosen to be 3 and 13 units. At position 15 of the fatty acid $\left(\mathrm{C}_{24: \Delta}\right)$, a double bond was introduced to check the effect of unsaturated fatty acids $\left(\mathrm{C}_{24: 1}\right)$ compared to saturate fatty acids $\left(\mathrm{C}_{24: 0}\right)$. The $\alpha$-hydroxylation $(\mathrm{x})$ was set to non-hydroxylized $(\mathrm{H})$ or hydroxylized $(\mathrm{OH})$ fatty acids. Phase preference of the head group labeled $\mathrm{Gb}_{3} \mathrm{~s}$ was measured according to the methodology and techniques already described for the fatty acid labeled $\mathrm{Gb}_{3} \mathrm{~s}$ (chapter 3.4.1). GUVs composed of DOPC/SM/Chol/Gb 3 /Dy731-DOPE (39:39:20:1:1) were used. An overview of the results from these eight different head group labeled $\mathrm{Gb}_{3} \mathrm{~s}$ is shown in table 4.3. In the following section, different aspects of the chemical modifications and their influence on the phase preference are discussed in detail.

\section{Linker length}

Surprisingly, the size of the linker attached to the head group of a lipid alters the phase behavior of the fluorescently labeled lipid dramatically. $188 \mid 189$ 
Table 4.3.: Mean values of the liquid ordered phase distribution $\left(\% l_{0}\right)$ for the different head group labeled $\mathrm{Gb}_{3} \mathrm{~s}$ in GUVs composed of DOPC/SM $\mathrm{Sorc}_{\mathrm{p}} / \mathrm{Chol} / \mathrm{Gb}_{3} / \mathrm{Dy} 731-\mathrm{DOPE}$ (39:39:20:1:1). The errors are the standard deviation of the mean. $N$ in brackets is the number of analyzed profile lines. The number (No.) references the $\mathrm{Gb}_{3}$-species in table 4.4 .

\begin{tabular}{l|cc} 
& $\% l_{\mathrm{o}}(\mathrm{N})$ & No. \\
\hline $\mathrm{Gb}_{3} \mathrm{PEG}_{3} \mathrm{C}_{24: 0} \mathrm{H}$ & $0.41 \pm 0.07(2516)$ & $\mathbf{1}$ \\
$\mathrm{Gb}_{3} \mathrm{PEG}_{3} \mathrm{C}_{24: 0} \mathrm{OH}$ & $0.42 \pm 0.08(2273)$ & $\mathbf{2}$ \\
$\mathrm{Gb}_{3} \mathrm{PEG}_{3} \mathrm{C}_{24: 1} \mathrm{H}$ & $0.32 \pm 0.07(2351)$ & $\mathbf{3}$ \\
$\mathrm{Gb}_{3} \mathrm{PEG}_{3} \mathrm{C}_{24: 1} \mathrm{OH}$ & $0.27 \pm 0.06(2701)$ & $\mathbf{4}$ \\
\hline $\mathrm{Gb}_{3} \mathrm{PEG}_{13} \mathrm{C}_{24: 0} \mathrm{H}$ & $0.74 \pm 0.07(2525)$ & $\mathbf{5}$ \\
$\mathrm{Gb}_{3} \mathrm{PEG}_{13} \mathrm{C}_{24: 0} \mathrm{OH}$ & $0.71 \pm 0.05(3064)$ & $\mathbf{6}$ \\
$\mathrm{Gb}_{3} \mathrm{PEG}_{13} \mathrm{C}_{24: 1} \mathrm{H}$ & $0.47 \pm 0.15(1654)$ & $\mathbf{7}$ \\
$\mathrm{Gb}_{3} \mathrm{PEG}_{13} \mathrm{C}_{24: 1} \mathrm{OH}$ & $0.50 \pm 0.08(2377)$ & $\mathbf{8}$
\end{tabular}

$\mathrm{Gb}_{3} \mathrm{~s}$ with saturated non-hydroxylated chains showed a clear $l_{\mathrm{d}}$ phase preference when a $\mathrm{PEG}_{3}$ linker was present $\left(\% l_{\mathrm{o}}=0.41 \pm 0.07\right.$, table 4.3$)$ and a strong $l_{\mathrm{o}}$ phase preference when $\mathrm{PEG}_{13}$ was used linker $\left(\% l_{\mathrm{o}}=0.74 \pm 0.07\right.$, (figure $4.9 \mathrm{~A}$, table 4.3 ). The difference of $0.33 \pm 0.14$ showed a significant change in the phase preference between these derivatives (table $4.4 \triangle \mathrm{PEG}$ first row). In the same manner, saturated and hydroxylated fatty acid derivatives (figure $4.9 \mathrm{~B}$ ) show an increase of $0.29 \pm 0.13$ in the $\% l_{\mathrm{o}}$ if the linker length was changed from 3 to 13 PEG units (table $4.4 \triangle \mathrm{PEG}$ second row). The effect of PEG linker to the phase preference is also present if unsaturated fatty acids were used without or with hydroxylation (figure 4.9 $\mathrm{C}$ and $\mathrm{D}$, table $4.4 \triangle \mathrm{PEG}$ last two rows). In conclusion, there is an average increase in the $\% l_{\mathrm{o}}$ of 0.25 from the short to the long PEG linker, independent of further chemical modifications. The influence of the linker length to the phase preference of the head group labeled $\mathrm{Gb}_{3} \mathrm{~s}$ was also analyzed with a linear mixed-effects model (LMM) to check on statistical significance (chapter A.7.2). The LMM analyzed all eight datasets of the eight different head group labeled $\mathrm{Gb}_{3} \mathrm{~s}$ at once. The LMM results in a significant influence of the PEG linker on the phase distribution from the head group labeled $\mathrm{Gb}_{3} \mathrm{~s}$. The $\mathrm{PEG}_{13}$ derivatives are preferentially located by the value of $0.27 \pm 0.03$ in the $l_{\text {o }}$ phase, as their correspondent $\mathrm{Gb}_{3}$-species with a $\mathrm{PEG}_{3}$ linker (table A.5).

The head group labeled $\mathrm{Gb}_{3} \mathrm{~s}$ with the longer linker length are preferentially lo-

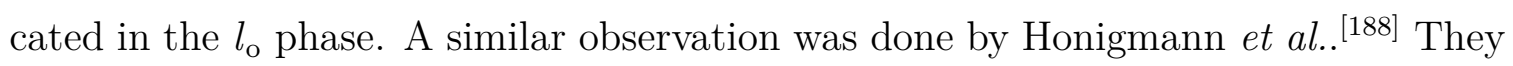
reported on a fluorophore that was connected either directly to the lipid DSPE or connected via a PEG-linker with 45 ethylene glycol units to the lipid. The fluorophore was reconstituted into supported lipid membranes composed of DPhPC/DPPC/Chol. 

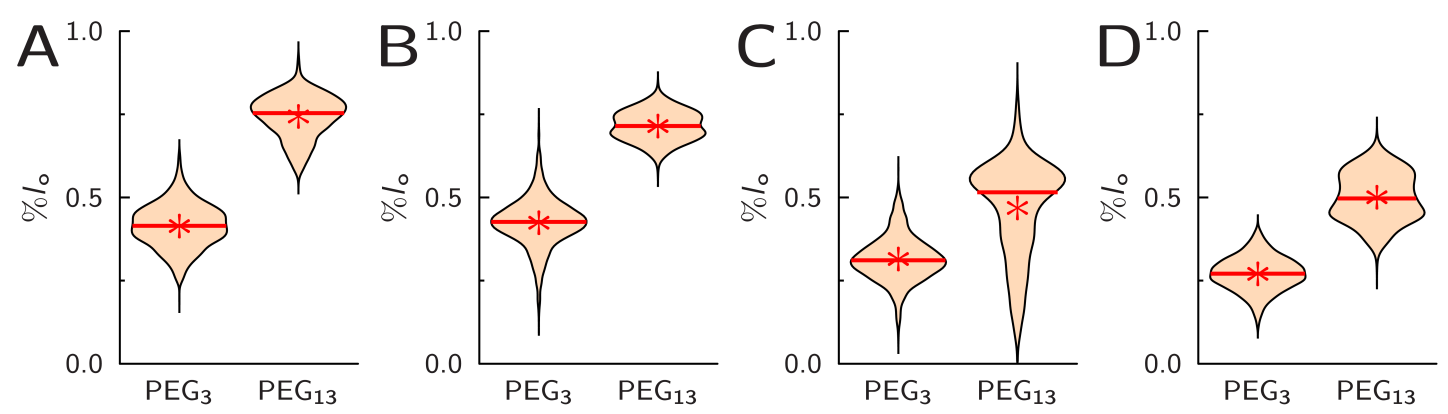

Figure 4.9.: Violin plots of the liquid ordered phase distribution $\left(\% l_{\mathrm{o}}\right)$ of different head group labeled globotriaosyl ceramide $\left(\mathrm{Gb}_{3}\right)$, which compare the different PEG linker length short $\left(\mathrm{PEG}_{3}\right)$ to long $\left(\mathrm{PEG}_{13}\right)$. The residue of the $\mathrm{Gb}_{3}$ structure is in $\mathrm{A} \mathrm{Gb}_{3} \mathrm{C}_{24: 0} \mathrm{H}$, in $\mathrm{B} \mathrm{Gb}_{3} \mathrm{C}_{24: 0} \mathrm{OH}$, in $\mathrm{C} \mathrm{Gb}_{3} \mathrm{C}_{24: 1} \mathrm{H}$ and in $\mathrm{D} \mathrm{Gb}_{3} \mathrm{C}_{24: 1} \mathrm{OH}$. The GUV is composed of DOPC/ $\mathrm{SM}_{\text {porc }} / \mathrm{Chol} / \mathrm{Gb}_{3} /$ Dy731-DOPE (39:39:20:1:1). The star represents the mean value. The median is given by the stroke. The mean values with their standard deviation are listed with the number of measurements in table 4.3 .

Table 4.4.: Differences in the mean values dependent on the functional group. The numbers are explained in table 4.3 and shorten the $\mathrm{Gb}_{3}$ species. $\Delta \mathrm{PEG}=\% l_{\mathrm{o}}\left(\mathrm{PEG}_{13}\right)-\% l_{\mathrm{o}}\left(\mathrm{PEG}_{3}\right)$; $\Delta \mathrm{C}_{24}=\% l_{\mathrm{o}}\left(\mathrm{C}_{24: 0}\right)-\% l_{\mathrm{o}}\left(\mathrm{C}_{24: 1}\right) ; \Delta \mathrm{OH}=\% l_{\mathrm{o}}(\mathrm{H})-\% l_{\mathrm{o}}(\mathrm{OH})$.

\begin{tabular}{ll||ll||rr}
\multicolumn{2}{c}{$\Delta$ PEG } & \multicolumn{2}{c}{$\Delta \mathrm{C}_{24}$} & \multicolumn{1}{c}{$\Delta \mathrm{OH}$} \\
\hline $\mathbf{5 - 1}$ & $0.33 \pm 0.14$ & $\mathbf{5 - 7}$ & $0.27 \pm 0.22$ & $\mathbf{5 - 6}$ & $0.03 \pm 0.13$ \\
$\mathbf{6 - 2}$ & $0.29 \pm 0.13$ & $\mathbf{6 - 8}$ & $0.21 \pm 0.13$ & $\mathbf{7 - 8}$ & $-0.03 \pm 0.23$ \\
$\mathbf{7 - 3}$ & $0.15 \pm 0.22$ & $\mathbf{1 - 3}$ & $0.09 \pm 0.14$ & $\mathbf{1 - 2}$ & $-0.01 \pm 0.15$ \\
$\mathbf{8 - 4}$ & $0.23 \pm 0.14$ & $\mathbf{2 - 4}$ & $0.15 \pm 0.14$ & $\mathbf{3 - 4}$ & $0.05 \pm 0.13$
\end{tabular}

A fluorescence analysis of the partition clearly showed that the fluorescent lipid lacking the PEG-linker was preferentially localized in the $l_{\mathrm{d}}$ phase, while that with the PEG-linker partitioned into the $l_{\mathrm{o}}$ phase. 188 The phase preference of fluorescently marked lipids was systematically analyzed by changing the PEG linker length, from 3 to 15 units, between the fluorophore and lipid. $189 \mid 190$ The used lipids for labeling are expected to be localized into the $l_{\mathrm{o}}$ phase, of coexisting $l_{\mathrm{o}} / l_{\mathrm{d}}$ phase-separated membranes. $189 \mid 190$ It was found that marked lipids are located in the $l_{\mathrm{d}}$ phase, if only 3 PEG units as linker were used, while, in contrast, the use of 15 PEG units as linker

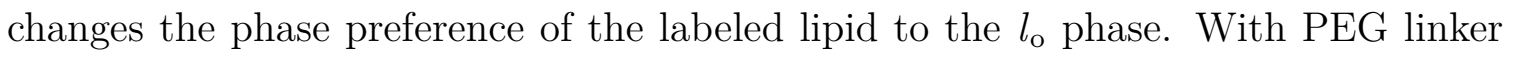
lengths of 5 and 10, they showed systematically the correlation between the increase of the PEG linker length and an increase of the $l_{\mathrm{o}}$ phase preference from the labeled lipid. $189[190$

This observation is explained by a model from Klymchenko et al..140] The fluorophore connected to the lipid is partially hydrophobic and might be also bulky. The fluorophore changes the packing parameter of the lipid, because it increases the head 
group size of the labeled lipid. If the distance from the fluorophore to the lipid was increased by a PEG linker, the influence of the fluorophore to the packing density of the lipid can be weaker, up to being not relevant at all.140

In our study, a 13-unit long linker decoupled the fluorophore from the membrane interface with the result that $\mathrm{Gb}_{3} \mathrm{PEG}_{13} \mathrm{C}_{24: 0} \mathrm{H}$ which is expected to at least preferentially partition into the $l_{\mathrm{o}}$ phase indeed has a $l_{\mathrm{o}}$ distribution of almost 0.75 . From these results, it can be conclude that the $\mathrm{Gb}_{3}$ species with $\mathrm{PEG}_{13}$ are better suited to report on the natural partition of $\mathrm{Gb}_{3}$ than those with $\mathrm{PEG}_{3}$. Thus, the experiments in which the influence of unsaturation and hydroxylation of the fatty acid of $\mathrm{Gb}_{3}$ is compared are all performed with the $\mathrm{PEG}_{13}$ species. The corresponding results with the $\mathrm{PEG}_{3}$ linker are plotted for the completeness in figures 4.10, 4.11 and 4.12 and are listed in tables 4.4 and 4.5 .

\section{Fatty acid saturation}

The influence of fatty acid saturation on the partition behavior of $\mathrm{Gb}_{3}$ was investigated (figure 4.10). The results show that introducing a fatty acid with a cis-double bond redistributes the $\mathrm{Gb}_{3}$ sphingolipid in the $l_{\mathrm{d}}$ phase which can be rationalized by the increased space requirement of the $\mathrm{Gb}_{3}$ species with the $\mathrm{C}_{24: 1}$ fatty acid. The differences between the $l_{\mathrm{o}}$ distribution of $\mathrm{Gb}_{3} \mathrm{PEG}_{13} \mathrm{C}_{24: 0} \mathrm{H} / \mathrm{Gb}_{3} \mathrm{PEG}_{13} \mathrm{C}_{24: 1} \mathrm{H}$ and $\mathrm{Gb}_{3} \mathrm{PEG}_{13} \mathrm{C}_{24: 0} \mathrm{OH} / \mathrm{Gb}_{3} \mathrm{PEG}_{13} \mathrm{C}_{24: 1} \mathrm{OH}$ harboring the $\mathrm{PEG}_{13}$ linker are significant and range between $0.21 \pm 0.13$ to $0.27 \pm 0.22$, respectively (table $4.4, \Delta \mathrm{C}_{24}$ ). The influence of saturation of the fatty acid on the phase preference was also statistically analyzed using the LMM. The analysis was done only for long linker length, these are obviously the better biological mimic model. $\mathrm{Gb}_{3} \mathrm{~s}$ with saturated fatty acids are highly present in the $l_{\mathrm{o}}$ phase than the $\mathrm{Gb}_{3} \mathrm{~s}$ with the unsaturated fatty acid (0.24 \pm 0.04 , table A.6).

The influence of the fatty acid on the lipid packing of glycosphongolipids with different saturated fatty acid was checked by Stefaniu et al. using a Langmuir-Blodgett technique. 191 The glycosphongolipids with the unsaturated fatty acid have $2 \%$ higher molecular area than the glycosphingolipids with the saturated fatty acid. The surprisingly small differences in area per lipid is explained by the strong head group interaction, which suppresses the effect of the unsaturated fatty acid, 191 as these measurements were performed at a surface pressure of $30 \mathrm{mN} \mathrm{m}^{-1}$ which reflects the packing density of lipid bilayers. 192

A bilayer study which contains glycosphingolipids with the fatty acids $\mathrm{C}_{18: 0}$ and $\mathrm{C}_{18: 1}$ was analyzed with deuterium nuclear magnetic resonance $\left({ }^{2} \mathrm{H}-\mathrm{NMR}\right) .193$ The 

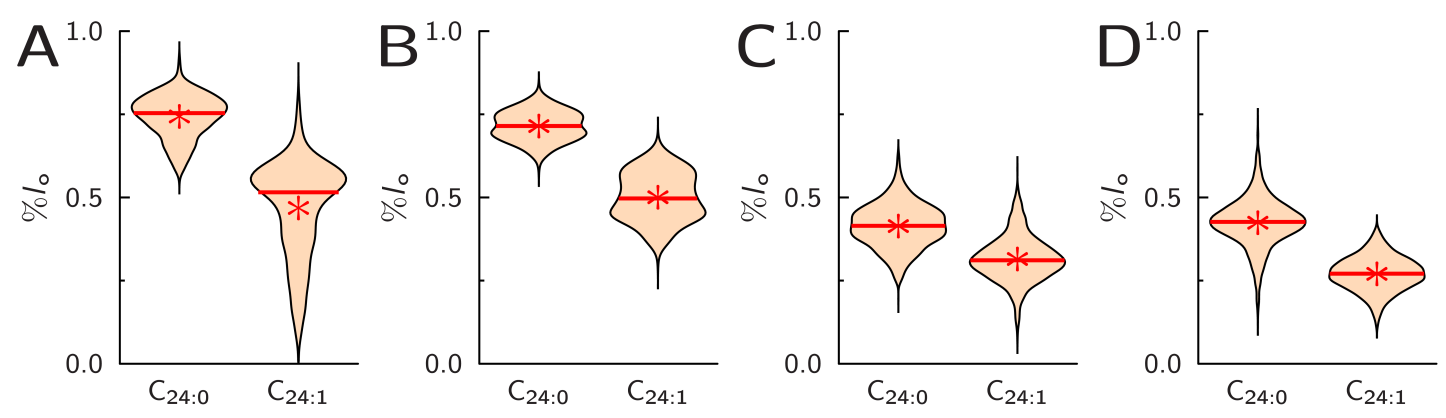

Figure 4.10.: The liquid ordered phase distribution $\left(\% l_{\mathrm{o}}\right)$ of head group labeled globotriaosyl ceramide $\left(\mathrm{Gb}_{3}\right)$. The saturated fatty acid $\left(\mathrm{C}_{24: 0}\right)$ were compared to the unsaturated fatty acid $\left(\mathrm{C}_{24: 1}\right)$ of the $\mathrm{Gb}_{3}$-species. The $\mathrm{Gb}_{3}$ residue in $\mathrm{A}$ is $\mathrm{Gb}_{3} \mathrm{PEG}_{13} \mathrm{H}$, in $\mathrm{B} \mathrm{Gb}_{3} \mathrm{PEG}_{13} \mathrm{OH}$, in $\mathrm{C} \mathrm{Gb}_{3} \mathrm{PEG}_{3} \mathrm{H}$ and in $\mathrm{D}_{\mathrm{Gb}_{3}} \mathrm{PEG}_{3} \mathrm{OH}$. The GUV composition was $\mathrm{DOPC} / \mathrm{SM}_{\text {porc }} / \mathrm{Chol} /$ $\mathrm{Gb}_{3} /$ Dy731-DOPE (39:39:20:1:1). The violin plots include the mean value as star and the median as stroke. The mean values with their standard deviation are listed with the number of measurements in table 4.3 .

advantage of the ${ }^{2} \mathrm{H}-\mathrm{NMR}$ study is that the ordering parameter is directly measurable and it shows that an unsaturated fatty acid connected to a glycoshingolipid has a less order parameter compared to the saturated fatty acid species. This indicates that unsaturated fatty acids are less incorporated into $l_{\mathrm{o}}$ phases in $l_{\mathrm{o}} / l_{\mathrm{d}}$ membranes, because the $l_{\mathrm{o}}$ phase consists of the ordered lipids. 194

Björkqvist et al.128 analyzed the transition temperature $\left(T_{\mathrm{M}}\right)$ of glycosphingolipids in bilayers by differential scanning calorimetry (DSC) measurements and by anisotropy measurements. The structures contain either saturated fatty acid $\left(\mathrm{C}_{24: 0}\right)$ or unsaturated fatty acid $\left(\mathrm{C}_{24: 1}\right)$. In all cases, the saturated system has a $\sim 20 \mathrm{~K}$ higher $T_{\mathrm{M}}$ compared to the unsaturated one. 128 The differences in the $T_{\mathrm{M}}$ for the $\mathrm{SM} \mathrm{C}_{24: 0}$ and $\mathrm{SM} \mathrm{C}_{24: 1}$ are also $\sim 20 \mathrm{~K}$ (table 3.2 ). $128 \mid 131,133$ The reduced $T_{\mathrm{M}}$ indicates lower packing density which results in less incorporation into the $l_{\mathrm{o}}$ phase. The incorporation of $\mathrm{SM} \mathrm{C}_{24: 1}$ into the $l_{\mathrm{o}}$ phase was measured by the property to create a $l_{\mathrm{o}}$ phase in DOPC/SM/Chol membranes. 195 . While $\mathrm{SM} \mathrm{C}_{24: 0}$ showed phase-separated membrane systems using this lipid mixture, the $\mathrm{SM} \mathrm{C}_{24: 1}$ resulted in a homogeneous membrane. This proves directly that the saturated fatty acid prefers the $l_{\mathrm{o}}$ phase in comparison to the unsaturated fatty acid.

The effect of fatty acid saturation was also found by doing lipid extraction studies of pHBMEC.46. DRM extraction was done to distinguish between the raft domains (DRM fraction) and fluid membrane (non-DRM fraction). 174 176 The $\mathrm{Gb}_{3} \mathrm{~S}$ were extracted from pHBMEC, the $\mathrm{Gb}_{3} \mathrm{~s}$ with the saturated fatty acids $\mathrm{C}_{24: 0}$ were enriched in the DRM fraction and the $\mathrm{Gb}_{3} \mathrm{~s}$ with the unsaturated fatty acids $\mathrm{C}_{24: 1}$ were enriched in the non-DRM fraction 46 which is in agreement with the study in this thesis. 


\section{$\alpha$-Hydroxylation}

In nature, there is a great amount of $\alpha$-hydroxylated fatty acids among the $\mathrm{Gb}_{3} \mathrm{~s} .185$ The influence of the hydroxylation in the fatty acids from the $\mathrm{Gb}_{3} \mathrm{~S}$ is shown in figure 4.11. No significant differences were found when non-hydroxylated $\mathrm{Gb}_{3} \mathrm{~s}$ are compared to hydroxylated $\mathrm{Gb}_{3} \mathrm{~S}$ (table $4.4 \Delta \mathrm{OH}$ ). The analysis of the LMM to the hydroxylation results in no significant differences between the head-group labeled $\mathrm{Gb}_{3} \mathrm{~s}(0.01 \pm 0.03 \mathrm{p}-$ Value $=0.67$, table A.5).
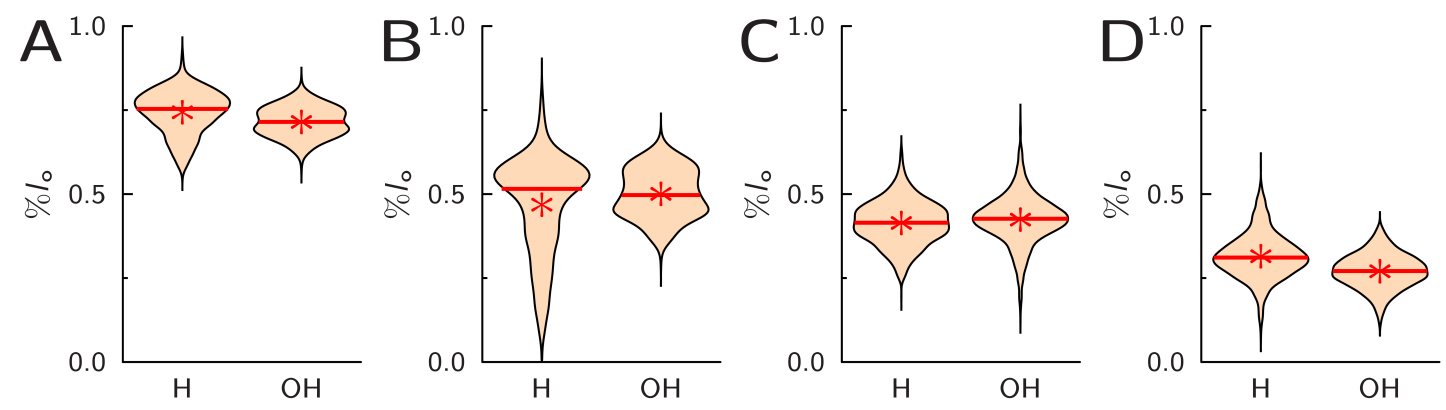

Figure 4.11.: The liquid ordered phase distribution $\left(\% l_{\mathrm{o}}\right)$ of the different head group labeled $\mathrm{Gb}_{3}$-species are compared to their hydroxylation in $\alpha$ position at the fatty acid in violin plots. The lipid composition of the GUVs was DOPC/SM $\mathrm{Sm}_{\text {porc }} / \mathrm{Chol} / \mathrm{Gb}_{3} / \mathrm{Dy} 731-\mathrm{DOPE}$ (39: 39:20:1:1). The $\mathrm{Gb}_{3}$ structures $\mathrm{Gb}_{3} \mathrm{PEG}_{13} \mathrm{C}_{24: 0}(\mathrm{~A}), \mathrm{Gb}_{3} \mathrm{PEG}_{13} \mathrm{C}_{24: 1}$ (B), $\mathrm{Gb}_{3} \mathrm{PEG}_{3} \mathrm{C}_{24: 0}$ (C) and $\mathrm{Gb}_{3} \mathrm{PEG}_{3} \mathrm{C}_{24: 1}$ (D) are plotted respectively. The mean values are given by a star and the median values are given as a stroke. The mean values with their standard deviation are listed with the number of measurements in table 4.3

The influence of $\alpha$-hydroxylation to the area per lipid was analyzed using galactosylceramide (GalCer) $\mathrm{C}_{24: 0}$ at an air water interface.191 Stefaniu et al. found that the $\alpha$-hydroxylated GalCer-species decrease the area per lipid with higher surface pressure. The influence of the surface pressure on the area per lipid from the hydroxylated species is a result of changing their fatty acid tilt angle depending on the surface pressure. In contrast, the non-hydroxylated GalCer has a constant area per lipid and a constant tilt angle during the change of the surface pressure. At a surface pressure of $30 \mathrm{mN} \mathrm{m}^{-1}$ which reflects the packing density of a bilayer, 192 both lipids have the same area per lipid.191 Therefore, no differences in lipid packing behavior are present which explains the finding that hydroxylation has no influence on the phase distribution of the $l_{\mathrm{o}} / l_{\mathrm{d}}$ phase.

Morrow et al. measured the difference of the hydroxylation in $\alpha$ position at the fatty acid $\mathrm{C}_{18: 0}$ from glycosphingolipids in POPC/Chol membranes with the ${ }^{2} \mathrm{H}$ NMR. They reported that the ordering parameter showed no differences for the $\alpha-$ hydroxylated and non-hydroxylated $\mathrm{C}_{18}$ at the glycosphingolipid. 193 The orientation of the sugar head group was also the same for both glycosphingolipids. 196 . This is 
in line with the observation that the hydroxyl-group did not significantly alter the partition of the $\mathrm{Gb}_{3}$-species in phase-separated GUVs.

However, even if the partitioning of $\mathrm{Gb}_{3}$ in the $l_{\mathrm{o}}$ phase is similar for the nonhydroxylated and hydroxylated species, Schütte et al. found that the 2-OH group influences the fraction of $l_{\text {o }}$ phase in phase-separated supported lipid bilayers. ${ }^{162}$ In case of the hydroxylated $\mathrm{C}_{24: 0}$ fatty acid, the $l_{\mathrm{o}}$ fraction is lower compared to the non hydroxylated species. $\frac{162}{2}$ Ekholm et al. showed that the 2-OH group increases the hydration in the membrane interface and decreases the affinity of a sphingolipid for sterols. 197 The same was found by Lingwood et al. 198 and Yahi et al.199 and

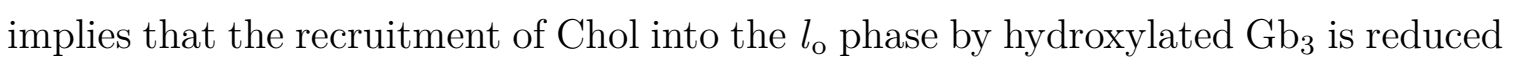
compared to the non-hydroxylated species leading to a smaller $l_{\mathrm{o}}$ fraction.

\section{Influence of the sphingomyelin fatty acid}

The studies of the influence from chemical differences on the head group labeled $\mathrm{Gb}_{3}$ s were done with a nature extraction of $\mathrm{SM}$, the $\mathrm{SM}_{\text {porc }}$. This $\mathrm{SM}$ is a mixture of different SMs, which are listed in table 3.1. The phase behavior of ternary mixtures are influenced by the lipids. The exchange of the sphingomyelin in a ternary lipid mixture from a SM mixture to a defined SM is known to alter the phase separation behavior. 200 Five different SM species with a saturated fatty acid varying in length were chosen, namely palmitoyl $\mathrm{SM}\left(\mathrm{SM} \mathrm{C}_{16: 0}\right)$, stearoyl $\mathrm{SM}\left(\mathrm{SM} \mathrm{C}_{18: 0}\right)$, arachidoyloyl $\mathrm{SM}\left(\mathrm{SM} \mathrm{C}_{20: 0}\right)$, behenoyl $\mathrm{SM}\left(\mathrm{SM} \mathrm{C}_{22: 0}\right)$ and lignoceroyl $\mathrm{SM}\left(\mathrm{SM} \mathrm{C}_{24: 0}\right)$.

For each of the eight different head group labeled $\mathrm{Gb}_{3}$-species, a $\% l_{\mathrm{o}}$ distribution was measured with each of the five different SMs (figure 4.12, table 4.5). Comparing the four $\mathrm{Gb}_{3}$-species with the $\mathrm{PEG}_{13}$ linker (figure $4.12 \mathrm{~A}, \mathrm{~B}, \mathrm{C}, \mathrm{D}$, table 4.5 first four rows) the $\% l_{0}$ increases with the length of the $\mathrm{SMs}$. From $\mathrm{SM} \mathrm{C}_{16: 0}$ to $\mathrm{SM} \mathrm{C}_{18: 0}$ there is a slide increase in the $\% l_{0}$ with the saturated fatty acid (figure $4.12 \mathrm{~A}$ and $\mathrm{B})$ and with the unsaturated fatty acid there is nearly no increase (figure $4.12 \mathrm{C}$ and $\mathrm{D})$. The strongest increase in $\% l_{\mathrm{o}}$ is found comparing $\mathrm{SM} \mathrm{C}_{18: 0}$ to $\mathrm{SM} \mathrm{C}_{20: 0}$. From $\mathrm{SM} \mathrm{C}_{20: 0}$ over $\mathrm{SMC}_{22: 0}$ to $\mathrm{SM} \mathrm{C}_{24: 0}$ there is only a minor or no increase in $\% l_{\mathrm{o}}$ for the $\mathrm{Gb}_{3} \mathrm{~S}$ with the $\mathrm{PEG}_{13}$ linker. The $\% l_{\mathrm{o}}$ data from the head group labeled $\mathrm{Gb}_{3} \mathrm{~s}$ with $\mathrm{PEG}_{13}$ linker was statistically analyzed with LMM. The influence of the fatty acid length from the $\mathrm{SMs}$ to the $\% l_{\mathrm{o}}$ of the $\mathrm{Gb}_{3}$ shows three groups, which significantly differ. The data of the $\mathrm{SM} \mathrm{C}_{20: 0}, \mathrm{SM} \mathrm{C}_{22: 0}$ and $\mathrm{SM} \mathrm{C}_{24: 0}$ showed no differences to each other. They differs significantly to the $\mathrm{SMC}_{16: 0}$ and $\mathrm{SMC}_{18: 0}$. The measured $\% l_{\mathrm{o}}$ with the $\mathrm{SM} \mathrm{C}_{16: 0}$ differs from the $\mathrm{SM} \mathrm{C}_{18: 0}$ (table A.8). 
4. Phase behavior of globotriaosyl ceramide $\left(\mathrm{Gb}_{3}\right)$


Figure 4.12.: The liquid ordered phase distribution $\left(\% l_{0}\right)$ was measured for all eight different head group labeled $\mathrm{Gb}_{3} \mathrm{~s} \mathrm{~Gb}_{3} \mathrm{PEG}_{13} \mathrm{C}_{24: 0} \mathrm{H}(\mathrm{A}), \mathrm{Gb}_{3} \mathrm{PEG}_{13} \mathrm{C}_{24: 0} \mathrm{OH}$ (B), $\mathrm{Gb}_{3} \mathrm{PEG}_{13} \mathrm{C}_{24: 1} \mathrm{H}(\mathrm{C}), \mathrm{Gb}_{3} \mathrm{PEG}_{13} \mathrm{C}_{24: 1} \mathrm{OH}(\mathrm{D}), \mathrm{Gb}_{3} \mathrm{PEG}_{3} \mathrm{C}_{24: 0} \mathrm{H}(\mathrm{E}), \mathrm{Gb}_{3} \mathrm{PEG}_{3} \mathrm{C}_{24: 0} \mathrm{OH}$ $(\mathrm{F}), \mathrm{Gb}_{3} \mathrm{PEG}_{3} \mathrm{C}_{24: 1} \mathrm{H}(\mathrm{G})$ and $\mathrm{Gb}_{3} \mathrm{PEG}_{3} \mathrm{C}_{24: 1} \mathrm{OH}(\mathrm{H})$ with different sphingomyelins (Ms). The different $\mathrm{SMs}$ are palmitoyl sphingomyelin $\left(\mathrm{SM} \mathrm{C}_{16: 0}\right)$, stearoyl sphingomyelin $\left(\mathrm{SM} \mathrm{C}_{18: 0}\right)$, arachidoyl sphingomyelin $\left(\mathrm{SM} \mathrm{C}_{20: 0}\right)$, behenoyl sphingomyelin $\left(\mathrm{SM} \mathrm{C}_{22: 0}\right)$ and lignoceroyl sphingomyelin ( $\mathrm{SM} \mathrm{C}_{24: 0}$ ) in the GUV lipid composition DOPC/SM/Chol/Gb3/ Dy731-DOPE (39:39:20:1:1). The mean values of each distribution are represented in the violin plots by a star and and median by a stroke. The mean values with their standard deviation are listed with the number of measurements in table 4.5 .

56 
Table 4.5.: Mean values of the liquid ordered phase distribution $\left(\% l_{0}\right)$ for the different head group labeled $\mathrm{Gb}_{3} \mathrm{~s}$ with different sphingomyelins (SMs) in GUVs composed of DOPC/SM/ Chol/Gb 3 /Dy731-DOPE (39:39:20:1:1). The SMs are palmitoyl sphingomyelin ( $\left.\mathrm{SM} \mathrm{C}_{16: 0}\right)$, stearoyl sphingomyelin $\left(\mathrm{SM} \mathrm{C}_{18: 0}\right)$, arachidoyl sphingomyelin $\left(\mathrm{SM} \mathrm{C}_{20: 0}\right)$, behenoyl sphingomyelin $\left(\mathrm{SM} \mathrm{C}_{22: 0}\right)$ and lignoceroyl sphingomyelin $\left(\mathrm{SM} \mathrm{C}_{24: 0}\right)$. The errors are presented as standard deviation and the numbers of analyzed profile lines are in brackets.

\begin{tabular}{l|l|l|l|l|l}
$\% l_{\mathrm{o}}(\mathrm{N})$ & $\mathrm{SMC}_{16: 0}$ & $\mathrm{SMC}_{18: 0}$ & $\mathrm{SM} \mathrm{C}_{20: 0}$ & $\mathrm{SM} \mathrm{C}_{22: 0}$ & $\mathrm{SM} \mathrm{C}_{24: 0}$ \\
\hline $\mathrm{Gb}_{3} \mathrm{PEG}_{13} \mathrm{C}_{24: 0} \mathrm{H}$ & $0.41 \pm 0.11$ & $0.55 \pm 0.17$ & $0.74 \pm 0.05$ & $0.74 \pm 0.09$ & $0.79 \pm 0.09$ \\
& $(2392)$ & $(2986)$ & $(2397)$ & $(2077)$ & $(1707)$ \\
$\mathrm{Gb}_{3} \mathrm{PEG}_{13} \mathrm{C}_{24: 0} \mathrm{OH}$ & $0.45 \pm 0.10$ & $0.59 \pm 0.14$ & $0.71 \pm 0.05$ & $0.72 \pm 0.07$ & $0.77 \pm 0.07$ \\
& $(3232)$ & $(3396)$ & $(2414)$ & $(2893)$ & $(2509)$ \\
$\mathrm{Gb}_{3} \mathrm{PEG}_{13} \mathrm{C}_{24: 1} \mathrm{H}$ & $0.24 \pm 0.10$ & $0.28 \pm 0.12$ & $0.54 \pm 0.11$ & $0.45 \pm 0.15$ & $0.57 \pm 0.12$ \\
& $(2035)$ & $(1845)$ & $(2482)$ & $(2759)$ & $(1730)$ \\
$\mathrm{Gb}_{3} \mathrm{PEG}_{13} \mathrm{C}_{24: 1} \mathrm{OH}$ & $0.35 \pm 0.06$ & $0.34 \pm 0.08$ & $0.46 \pm 0.08$ & $0.51 \pm 0.09$ & $0.50 \pm 0.10$ \\
& $(1814)$ & $(2465)$ & $(2648)$ & $(2266)$ & $(2491)$ \\
\hline $\mathrm{Gb}_{3} \mathrm{PEG}_{3} \mathrm{C}_{24: 0} \mathrm{H}$ & $0.12 \pm 0.06$ & $0.14 \pm 0.07$ & $0.37 \pm 0.07$ & $0.36 \pm 0.06$ & $0.42 \pm 0.13$ \\
& $(1890)$ & $(1539)$ & $(2188)$ & $(2363)$ & $(1695)$ \\
$\mathrm{Gb}_{3} \mathrm{PEG}_{3} \mathrm{C}_{24: 0} \mathrm{OH}$ & $0.12 \pm 0.07$ & $0.12 \pm 0.07$ & $0.36 \pm 0.09$ & $0.36 \pm 0.09$ & $0.33 \pm 0.10$ \\
& $(2769)$ & $(2549)$ & $(2907)$ & $(2155)$ & $(2431)$ \\
$\mathrm{Gb}_{3} \mathrm{PEG}_{3} \mathrm{C}_{24: 1} \mathrm{H}$ & $0.05 \pm 0.04$ & $0.05 \pm 0.03$ & $0.20 \pm 0.08$ & $0.20 \pm 0.07$ & $0.10 \pm 0.06$ \\
& $(2384)$ & $(2413)$ & $(2227)$ & $(2259)$ & $(2505)$ \\
$\mathrm{Gb}_{3} \mathrm{PEG}_{3} \mathrm{C}_{24: 1} \mathrm{OH}$ & $0.19 \pm 0.12$ & $0.09 \pm 0.04$ & $0.32 \pm 0.09$ & $0.20 \pm 0.09$ & $0.20 \pm 0.06$ \\
& $(3086)$ & $(1768)$ & $(1988)$ & $(2685)$ & $(1828)$
\end{tabular}

The general trend, that the head group labeled $\mathrm{Gb}_{3} \mathrm{~s}$ are more incorporated in the $l_{\mathrm{o}}$ phase, if the fatty acid length of the SMs increases can be explained by the higher order of the SMs with increasing fatty acid length. The gel-liquid phase $T_{\mathrm{M}}$ increases with the fatty acid length from the SMs (table 3.2). 201. The higher order of the SMs with increasing the fatty acid length was also measured at the air-water interface. $131 \mid 202$

The strong increase of the $\% l_{\mathrm{o}}$ from $\mathrm{SMC}_{18: 0}$ to $\mathrm{SM} \mathrm{C}_{20: 0}$ is not described by the higher order of the SMs by increasing the fatty acid length. The increase of the fatty acid length in the SM increases the asymmetric chain lengths. 125 Bilayers of lipids with such asymmetric chain lengths tent to interdigitate into the other leaflet. ${ }^{126}$ This effect increases with higher asymmetry between the chains. $124 \mid 203$ The influence of the fatty acid length from SMs to the interdigitation was measured with X-Ray scattering in the gel-phase. $137 \mid 204$ The interdigitation was also observed for pure SMs with the fatty acids $\mathrm{C}_{20: 0}, \mathrm{C}_{22: 0}$ and $\mathrm{C}_{24: 0}$ in liquid crystalline phases. 205|206 The SMs with the long fatty acids interdigitate also in membranes with different lipid compositions which was shown by computer simulations. 207. The fatty acid mismatch of the head group labeled $\mathrm{Gb}_{3}$ species is comparable to the fatty acid mismatch found in $\mathrm{SM} \mathrm{C}_{24: 0}$. 
This explains why $\mathrm{Gb}_{3}$ s prefers interdigitated membrane areas which are present in membranes with SMs with fatty acids longer than or equal to $\mathrm{C}_{20: 0}$.

The incorporation of $\mathrm{Gb}_{3} \mathrm{~s}$ into the $l_{\mathrm{o}}$ phase can also be an effect of the Chol content in the $l_{\mathrm{o}}$ phase. It is known that Chol is better soluble in the SMs as in phosphatidylcholine (PC) membranes. $134|202| 208]$ Chol is best soluble in $\mathrm{SM} \mathrm{C}_{16: 0} \cdot 134 \mid 208$ If the Chol content in the $l_{\mathrm{o}}$ phase would have a greater influence as the SM-species, the $\% l_{\mathrm{o}}$ from the $\mathrm{SM} \mathrm{C}_{16: 0}$ is expected to be dramatically different from the residual chain lengths. Following the data in table 4.5, this is not the case, so the interaction of $\mathrm{Gb}_{3}$ to Chol appears to be less important than the interaction of $\mathrm{Gb}_{3}$ to $\mathrm{SM}$ for phase distribution of head group labeled $\mathrm{Gb}_{3} \mathrm{~s}$.

\section{Binding of STxB}

To check whether head group labeled $\mathrm{Gb}_{3} \mathrm{~S}$ are representing the natural $\mathrm{Gb}_{3} \mathrm{~S}$ in terms of phase behavior after STxB binding, Cy3 fluorescently marked STxB was used to perform binding studies. The $\mathrm{Gb}_{3}$-species with the $\alpha$-hydroxylated fatty acids were not analyzed, because the phase preference is identical to the phase preference of the $\mathrm{Gb}_{3}$-species with the non-hydroxylated fatty acids.

GUVs consisting of DOPC/SM ${ }_{\text {porc }} / \mathrm{Chol} / \mathrm{Gb}_{3} / \mathrm{Dy} 731-\mathrm{DOPE}$ (39:35:20:5:1) were incubated with $500 \mathrm{~nm}$ STxB Cy3 and afterwards imaged via confocal laser scanning microscope (CLSM). The quantitative analysis of the phase reference of head group labeled $\mathrm{Gb}_{3} \mathrm{~S}$ was not possible, since for STxB binding, concentrations of $\mathrm{Gb}_{3}$ in selfquenching amounts was necessary. The images are shown in figure 4.13. Using the fluorescence intensity of STxB Cy3 (blue) and the $l_{\mathrm{d}}$ fluorescent marker Dy731-DOPE (red), the localization of the $\mathrm{STxB} \mathrm{Gb}_{3}$ binding was investigated. Underneath each fluorescent image, the circular intensity profile of the GUV is plotted for a better comparison of the intensity information. Figure $4.13 \mathrm{~A}$ shows the $\mathrm{Gb}_{3} \mathrm{PEG}_{13} \mathrm{C}_{24: 0} \mathrm{H}$ which has the highest preference for the $l_{\mathrm{o}}$ phase, before STxB binds. The circular profile shows a decrease in the Dy731-DOPE intensity and at the same position an increase in the STxB Cy3 intensity. This means that both fluorophores are anti correlated and are located in different phases. While Dy731-DOPE marks the $l_{\mathrm{d}}$ phase, it is clear visible that the $\mathrm{STxB}$ binds into the $l_{\mathrm{o}}$ phase.

The change of the fatty acid from the saturated to the unsaturated one of the head group labeled $\mathrm{Gb}_{3}$ changes the phase preference of the STxB binding. Since, the unsaturated $\mathrm{Gb}_{3}$ does incorporate into the $l_{\mathrm{o}}$ phase (0.47), it is of interest how the distribution changes upon STxB binding. The circular profile line in figure $4.13 \mathrm{~B}$ shows a correlation between fluorescence intensities of STxB Cy3 and Dy731-DOPE. This cor- 

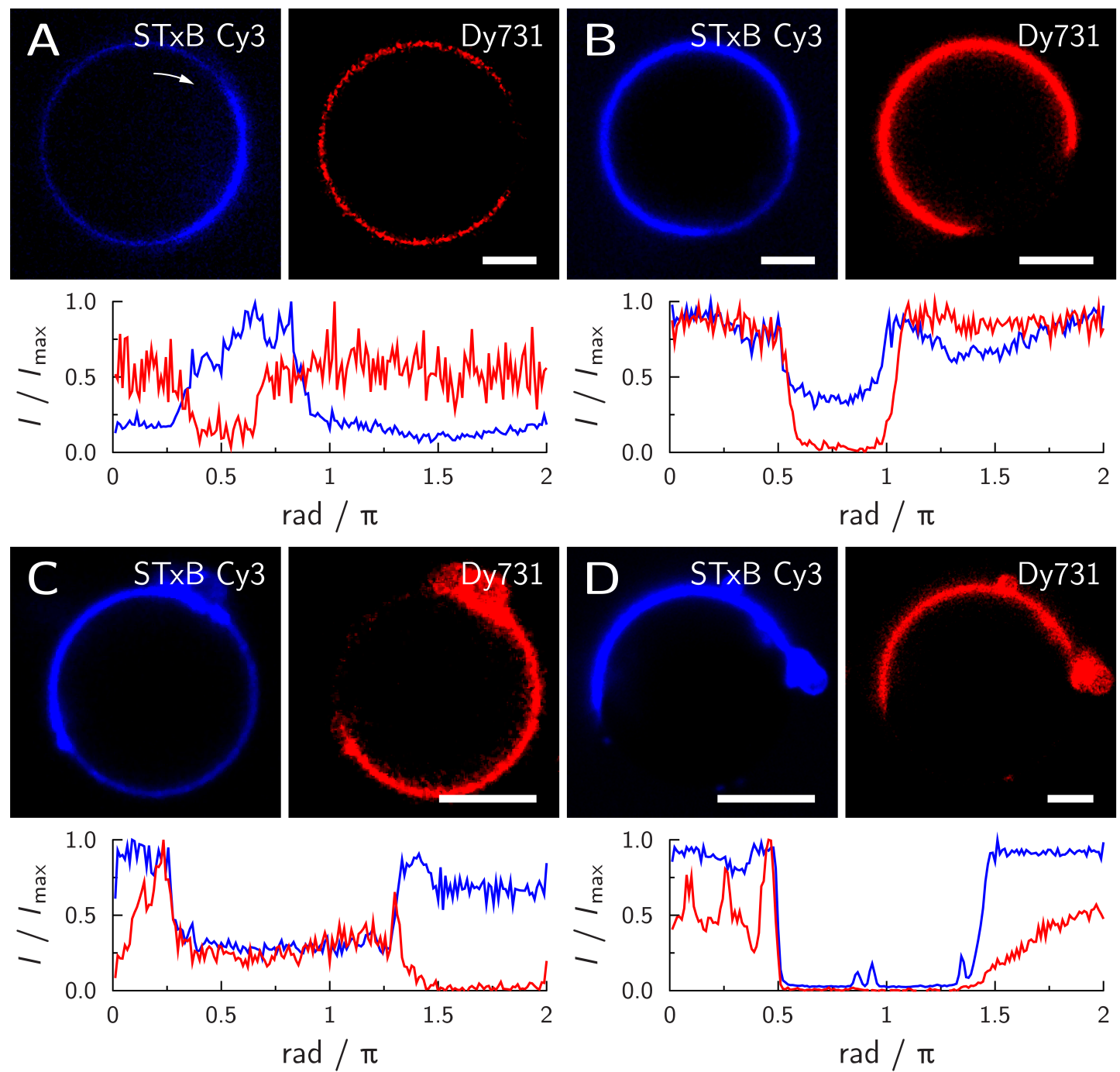

Figure 4.13.: Confocal images of phase-separated GUV composed of DOPC/SM $\mathrm{Sm}_{\text {porc }} / \mathrm{Chol} /$ $\mathrm{Gb}_{3} /$ Dy731-DOPE (39:35:20:5:1) in a $500 \mathrm{~nm}$ STxB Cy3 solution. The different head group labeled $\mathrm{Gb}_{3}$ s were $\mathrm{Gb}_{3} \mathrm{PEG}_{13} \mathrm{C}_{24: 0} \mathrm{H}(\mathrm{A}), \mathrm{Gb}_{3} \mathrm{PEG}_{13} \mathrm{C}_{24: 1} \mathrm{H}(\mathrm{B}), \mathrm{Gb}_{3} \mathrm{PEG}_{3} \mathrm{C}_{24: 0} \mathrm{H}(\mathrm{C})$ and $\mathrm{Gb}_{3} \mathrm{PEG}_{3} \mathrm{C}_{24: 1} \mathrm{H}(\mathrm{D})$. The labeled STxB is blue-colored and the Dy731-DOPE is shown in red. Under the fluorescent images, the normalized circular profile of the GUV from these two fluorophores are shown starting at twelve o'clock and going clockwise round the GUV, symbolized as a white arrow in A. The scale bars represent $5 \mu \mathrm{m}$. 
relation of the two fluorophores indicates that the $\mathrm{STxB}$ binding to $\mathrm{Gb}_{3} \mathrm{PEG}_{13} \mathrm{C}_{24: 1} \mathrm{H}$ takes place in the $l_{\mathrm{d}}$ phase.

Using unsaturated fatty acid derivates of $\mathrm{Gb}_{3}$, the $\mathrm{STxB} \mathrm{Gb}_{3}$ binding occurs a higher amount in the $l_{\mathrm{d}}$ phase (figure $4.13 \mathrm{~B}$ and $\mathrm{D}$ ). While before STxB binding the $\mathrm{Gb}_{3} \mathrm{PEG}_{13} \mathrm{C}_{24: 1} \mathrm{H}$ is distributed nearly $1: 1$ between $l_{\mathrm{o}}$ and $l_{\mathrm{d}}$ phase $\left(\% l_{\mathrm{o}}=\right.$ $0.47 \pm 0.15)$. The $\mathrm{STxB}$ binds the $\mathrm{Gb}_{3}$ to $\sim 0.33$ in the $l_{\mathrm{o}}$ phase. This indicated decrease is still in the measured errors. Schütte et al. found out that STxB binds $\mathrm{Gb}_{3}$ with the unsaturated fatty acid $\mathrm{C}_{24: 1}$ only in the $l_{\mathrm{o}}$ phase, 162 which is in contrast to our study with the $\mathrm{Gb}_{3} \mathrm{PEG}_{13} \mathrm{C}_{24: 1} \mathrm{H}$.

STxB $\mathrm{Gb}_{3}$ binding can be altered depending on the saturation state of the alkyl chains of the $\mathrm{Gb}_{3}$, when a long PEG linker is used. A preference of STxB to the $l_{\mathrm{o}}$ phase was found when saturated fatty acid were used and to the $l_{\mathrm{d}}$ phase when unsaturated fatty acid was used.

The $\mathrm{Gb}_{3} \mathrm{PEG}_{13} \mathrm{C}_{24: 0} \mathrm{H}$ is located before the $\mathrm{STxB}$ binding in the $l_{\mathrm{o}}$ phase with $0.74 \pm 0.07$ and the $\mathrm{STxB} \mathrm{Gb}_{3}$ binding is to $\sim 0.75$ in the $l_{\mathrm{o}}$ phase, indicated by the $\mathrm{STxB}$ intensity. This indicates no recruitment of the $\mathrm{Gb}_{3}$ through $\mathrm{STxB}$ which is in line with Schütte et al..162 They found that the $l_{\mathrm{o}}$ area did not increase on solid supported membranes (SSMs) composed of DOPC/SM $\mathrm{Sorc}_{\text {phol } / \mathrm{Cb}}$ (40:35:20:5), if the $\mathrm{STxB}$ binds to the $\mathrm{Gb}_{3}$ with a $\mathrm{C}_{24: 0}$ fatty acid. 162

To check the possibility that $\mathrm{STxB}$ recruits $\mathrm{Gb}_{3}$ into the $l_{\mathrm{o}}$ phase, $\mathrm{STxB} \mathrm{Cy} 3$ binding experiments were done with GUVs, containing $\mathrm{Gb}_{3}$-species with short $\mathrm{PEG}_{3}$ linker which means that the $\mathrm{Gb}_{3}$ prefers the $l_{\mathrm{d}}$ phase before STxB binding (figure 4.9). STxB binds the $\mathrm{Gb}_{3} \mathrm{PEG}_{3} \mathrm{C}_{24: 0} \mathrm{H}$ in the $l_{\mathrm{o}}$ phase, as indicated by the anti correlation in the circular profile of figure $4.13 \mathrm{C}$. The change of the fatty acid from the saturated to the unsaturated of the $\mathrm{Gb}_{3}$-species with the short PEG linker changes the phase preference of the STxB binding from the $l_{\mathrm{o}}$ to the $l_{\mathrm{d}}$ phase which is shown in the correlation of the two fluorophores in the circular profile in figure $4.13 \mathrm{D}$. The phase preference of $\mathrm{STxB}$ binding to the $\mathrm{Gb}_{3} \mathrm{PEG}_{3} \mathrm{C}_{24: 0} \mathrm{H}$ was found to be $\sim 0.7$. This indicates a high recruitment of the $\mathrm{Gb}_{3}$ s into the $l_{\mathrm{o}}$ phase, because the $\mathrm{Gb}_{3} \mathrm{PEG}_{3} \mathrm{C}_{24: 0} \mathrm{H}$ location is only up to $0.41 \pm 0.07$ in the $l_{\text {o }}$ phase before STxB binding. This recruitment of $\mathrm{Gb}_{3}$ into the $l_{\mathrm{o}}$ phase was precisely analyzed on pore-spanning membranes (PSMs) ${ }^{209}$ and in HeLa cells. 145 In HeLa cells, Falguières et al. analyzed the amount of $\mathrm{Gb}_{3}$ in the DRM before and after the STxB binding, which increases. 145 


\subsection{Conclusion of phase behavior of $\mathrm{Gb}_{3}$}

The bacterium enterohemorrhagic Escherichia coli (EHEC) is one of the main causes of food borne illness also present in industrial countries. The toxin of this bacteria is shiga toxin (STx) which inhibits the protein biosynthesis of eucaryotic cells. In a first step of approaching the cells, the B-subunit of STx (STxB) binds to the receptor lipid globotriaosyl ceramide $\left(\mathrm{Gb}_{3}\right)$. This receptor lipid is in different amounts present in the outer membrane of human cells. The cellular membrane is very heterogeneous and has regions of higher and lower ordering. It is known that binding of STx to the membrane takes place in a more ordered membrane structure. To get a better knowledge about the distribution of the $\mathrm{Gb}_{3}$ in the membrane before and after STxB binding, phase behavior of fluorescently labeled $\mathrm{Gb}_{3} \mathrm{~s}$ was analyzed in biomimetic model membranes.

The phase distribution behavior of fluorescently labeled $\mathrm{Gb}_{3}$ was studied in phaseseparated giant unilamellar vesicles (GUVs) composed of DOPC/SM $\mathrm{Sorc}_{\text {p }} /$ cholesterol (Chol). Two different labeling strategies were used: fatty acid labeled $\mathrm{Gb}_{3}$ and head group labeled $\mathrm{Gb}_{3}$. Two different fatty acid labeled $\mathrm{Gb}_{3}$ s were analyzed: $\mathrm{Gb}_{3}$ phenylmodified fatty acid $\left(\mathrm{Gb}_{3} \mathrm{PH}\right)$ and $\mathrm{Gb}_{3}$ thienothienyl-modified fatty acid $\left(\mathrm{Gb}_{3} \mathrm{TT}\right)$. $\mathrm{Gb}_{3} \mathrm{PH}$ was found to be nearly homogeneous distributed in the liquid ordered $\left(l_{\mathrm{o}}\right) /$ liquid disordered $\left(l_{\mathrm{d}}\right)$ phase-separated membrane before and after STxB binding. $\mathrm{Gb}_{3} \mathrm{TT}$ is only present at a partition of 0.24 in the $l_{\mathrm{o}}$ phase before and after STxB binding. STxB showed preferred binding to the $l_{\mathrm{d}}$ phase. This is in contradiction to the phase distribution of natural $\mathrm{Gb}_{3} \mathrm{STxB}_{\text {binding. } 54}$ Thus tail labeled $\mathrm{Gb}_{3}$-species are not suitable representations for the natural $\mathrm{Gb}_{3} \mathrm{~s}$.

A second strategy to label $\mathrm{Gb}_{3}$ is at the head group. The fluorophore BODIPY was connected with a polyethylene glycol (PEG) linker directly to the trisaccharide. This also allowed to vary the fatty acid of the labeled $\mathrm{Gb}_{3} \mathrm{~s}$. The major part of the $\mathrm{Gb}_{3} \mathrm{~S}$ in erythrcytes, $\frac{185}{1}$ HeLa cells 186 and HEp-2 cells 187 have a fatty acid length of 24 carbon atoms which was used in this thesis. The head group label allowed to study the effects of saturation and $\alpha$-hydroxylation of the chains on the phase distribution behavior before STxB binding.

First, it was found that changing the linker length from 3 to 13 PEG units changes the phase preference from $l_{\mathrm{d}}$ to $l_{\mathrm{o}}$ phase. Since the interaction between the membrane and the fluorophore decreases with increasing linker length, a longer linker is more suitable to represent the natural $\mathrm{Gb}_{3}$ behavior. $\mathrm{Gb}_{3}$-species with the unsaturated fatty acid preferred the $l_{\mathrm{d}}$ phase compared to the $\mathrm{Gb}_{3}$-species with the saturated fatty acid. Hydroxylation had no influence on the phase preference of the $\mathrm{Gb}_{3} \mathrm{~s}$. 
Furthermore, the influence of the lipid mixture on the phase preference of $\mathrm{Gb}_{3}$ was investigated. Five different sphingomyelins (SMs) were chosen to study the phase preference of $\mathrm{Gb}_{3}$. With increasing $\mathrm{SM}$ chain length, solubility of $\mathrm{Gb}_{3}$ in the $l_{\mathrm{o}}$ phase is also increasing.

Concerning STxB binding to $\mathrm{Gb}_{3}$, $\mathrm{STxB}$ binds to head group labeled $\mathrm{Gb}_{3}$, but a quantitative analysis was not possible, since for STxB binding concentrations of $\mathrm{Gb}_{3}$ in self quenching amounts were necessary prohibiting a quantitative analysis. The phase preference of STxB binding to head group labeled $\mathrm{Gb}_{3}$ ranges from the $l_{\mathrm{d}}$ phase to the $l_{\mathrm{o}}$ phase. Head group labeled $\mathrm{Gb}_{3} \mathrm{~s}$ are more suitable representations for the natural $\mathrm{Gb}_{3} \mathrm{~s}$ than fatty acid labeled $\mathrm{Gb}_{3} \mathrm{~S}$. 


\section{Pore-spanning membranes (PSMs)}

The cellular membrane is build up with different lipids and proteins and determines the shape of cells. The lipid raft theory says that there are different lipid order in the cellular membrane which are mimicked with the model of liquid ordered $\left(l_{\mathrm{o}}\right)$ /liquid disordered $\left(l_{\mathrm{d}}\right)$ phase-separated membranes.One important aspect in determining the shape of cells is the strength of the interactions between the membrane and cytoskeleton as well as between membrane and substrate. Examples of these are cell-cell junctions, 108 glycolipid domain adhesion, $210 \mid 211$ neuronal synapses 212 or the cytoskeleton. $\frac{87 \mid 213}{3}$ These interactions have in common, that the membrane is in an adhesive interaction with other structures and that these interactions strongly influence cellular shape, membrane properties like composition and mechanical stability. To mimic such a heterogeneously adhered membrane, a system with different membrane adhesion properties is required. Pore-spanning membranes (PSMs) are a suitable system to mimic different adhesion states and their influence on lipid membranes. In these models lipid membranes are spanned over nano- to micrometer large cavities, dividing the membrane into free-standing (non adhered) and solid supported (adhered) membrane areas (figure 5.1).

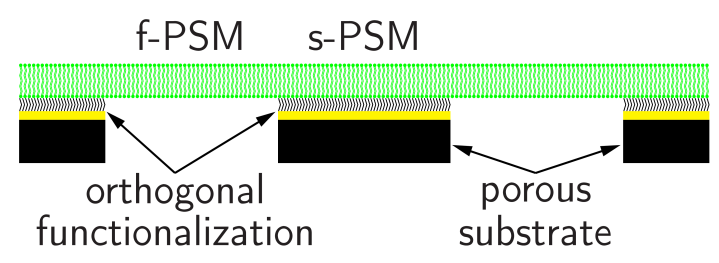

Figure 5.1.: The pore-spanning membrane (PSM) is generated on an orthogonal functionalized porous substrate. PSMs have two different membrane areas with different properties. Freestanding PSMs (f-PSMs) are spanned over the cavities. Solid supported PSMs (s-PSMs) are adhered to the functionalized surface.

With the PSMs, properties of membranes like membrane tension and lipid diffusion as well as their interplay can be studied to gain insights into their importance and effects in cellular systems.

The membrane tension strongly depends on the surface functionalization of the substrates. $\frac{214}{21}$ Therefore, different functionalization strategies were performed and 
the membrane tension was measured by means of atomic force microscopy (AFM) indentation experiments. 155 These tensions were compared with the membrane tension of cells which ranges from 0.01 to $0.15 \mathrm{mN} \mathrm{m}^{-1} .215 \mid 216$

In most common functionalization strategies a gold layer close to the membrane is included. The fluorescence emission is quenched on the s-PSM. 217 219 In this thesis, different functionalization strategies were studied regarding their fluorescence information. The silicon oxide $\left(\mathrm{SiO}_{\mathrm{x}}\right)$ functionalization allows to analyze $l_{\mathrm{o}} / l_{\mathrm{d}}$ phaseseparated membranes not only in the f-PSM, but also in the s-PSM. The influence of the two adhesion regimes were analyzed to the phase transition of the different lipid phases depending on the cholesterol (Chol) content.

Moreover, diffusion is an important aspect of different lipid species in the cell membrane. The diffusion constant of different lipids was measured in the PSM with interferometric scattering microscopy (iSCAT) or fluorescence correlation spectroscopy (FCS).

\subsection{Different functionalization strategies and their effects on pore-spanning membranes (PSMs)}

Various functionalization strategies were used to generate the PSMs. The functionalizations were mercaptoalcohols on gold, plasma cleaned silicon nitride $\left(\mathrm{p}-\mathrm{Si}_{3} \mathrm{~N}_{4}\right)$ or $\mathrm{SiO}_{\mathrm{x}}$. The $\mathrm{SiO}_{\mathrm{x}}$ functionalization was published in: Teske N., Sibold J., Schumacher J., Teiwes N. K., Gleisner M., Mey I. \& Steinem C. Continuous Pore-Spanning Lipid Bilayers On Silicon Oxide-Coated Porous Substrates. Langmuir 33, 14175-14183 (2017). The measurements of the lipid diffusion by iSCAT and of membrane tensions

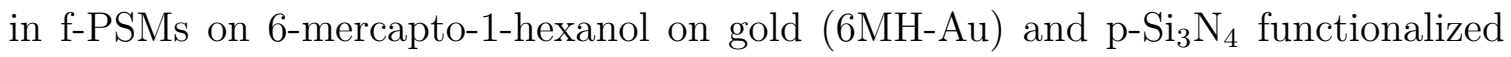
substrates were published in: Spindler S., Sibold J., Gholami Mahmoodabadi R.,

Steinem C. \& Sandoghdar V. High-Speed Microscopy of Diffusion in Pore-Spanning Lipid Membranes. Nano Lett. 18, 5262-5271 (2018).

Crucial for all functionalization methods is that the functionalization is only on the top of the porous substrates which were achieved with a orthogonal evaporation process. The orthogonal evaporation generated a cut off of functionalization at the pore border which is essential, because the membrane sheet, prepared by rupturing giant unilamellar vesicles (GUVs), can suspend over the pores. In contrast, if the cylindrical pore walls were also functionalized, the membrane typically adheres to the cylindrical pore walls and ruptures, instead of creating PSMs. Depending on the functionalization, different materials were used for the evaporation. An orthogonal 
gold layer was evaporated on the substrates for the mercaptoalcohols on gold and the p- $\mathrm{Si}_{3} \mathrm{~N}_{4}$ functionalization (chapter 3.2.2). Silicon monooxide ( $\mathrm{SiO}$ ) was orthogonally evaporated on the substrates to generate the $\mathrm{SiO}_{\mathrm{x}}$ functionalization (chapter 3.2.2).

The orthogonality of the evaporation for the two different evaporation processes was proven using scanning electron microscopy (SEM). The evaporated gold or $\mathrm{SiO}$ on top of the substrates was homogeneous (figure 5.2 A and C). A cross section of the orthogonal evaporated substrate shows the gold as well as the $\mathrm{SiO}_{\mathrm{x}}$ layer exclusively on top of the substrate (figure $5.2 \mathrm{~B}$ and $\mathrm{D}$ ). Small gold or $\mathrm{SiO}_{\mathrm{x}}$ deposits become discernible also at the inner parts of the cylindrical pore walls. A homogeneous and continuous surface coverage with only small deposits of the material inside the pores is required to induce GUV spreading to form PSMs. 214
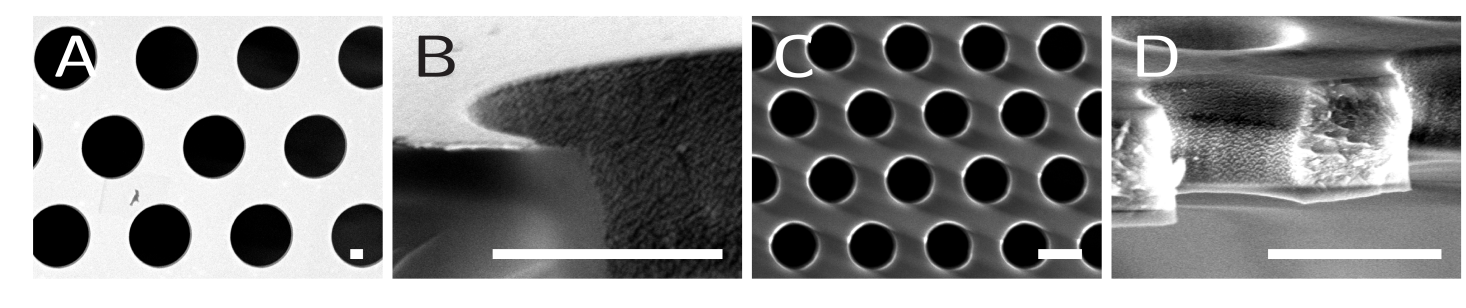

Figure 5.2.: The orthogonal evaporation of gold on porous substrates is shown in A and B. In $\mathrm{C}$ and $\mathrm{D}$ the evaporation of $\mathrm{SiO}$ is shown. $\mathrm{A}$ and $\mathrm{C}$ are top views which shows the homogeneity of both evaporation processes. B and D are cross sections which shows small clusters of the evaporation material in the pores. Scale bars $1 \mu \mathrm{m}$.

The gold and $\mathrm{SiO}$ evaporation processes resulted in a homogeneously covered layer on top of the substrates. Just small deposits were observed at the inner pore walls, indicating a successful functionalization to generate PSMs on the porous substrates.

\subsubsection{Visualization of pore-spanning membranes (PSMs)}

The silicon oxide surface was directly used to generate PSMs by spreading GUVs. The plasma cleaned silicon nitride surface was used to generate PSMs by spreading GUVs, after the cylindrical pore walls was passivated with polyethylene glycol (PEG). After successful physisorption of gold on the surface, chemisorption of thiols like mercaptoalcolhols was necessary to establish a surface vesicles can spread on.

A schematic illustration of the three different functionalization is shown in figure 5.3 (top row). All three surface functionalizations resulted in a hydrophilic surface which is covered with hydoxyl-groups. In detail, the surface of $6 \mathrm{MH}-\mathrm{Au}$ is full of hydroxylgroups from the $6 \mathrm{MH}$. The functionalization $\mathrm{p}-\mathrm{Si}_{3} \mathrm{~N}_{4}$ and $\mathrm{SiO}_{\mathrm{x}}$ resulted in a surface with silicon hydroxyl-groups. $148|220| 221$ 

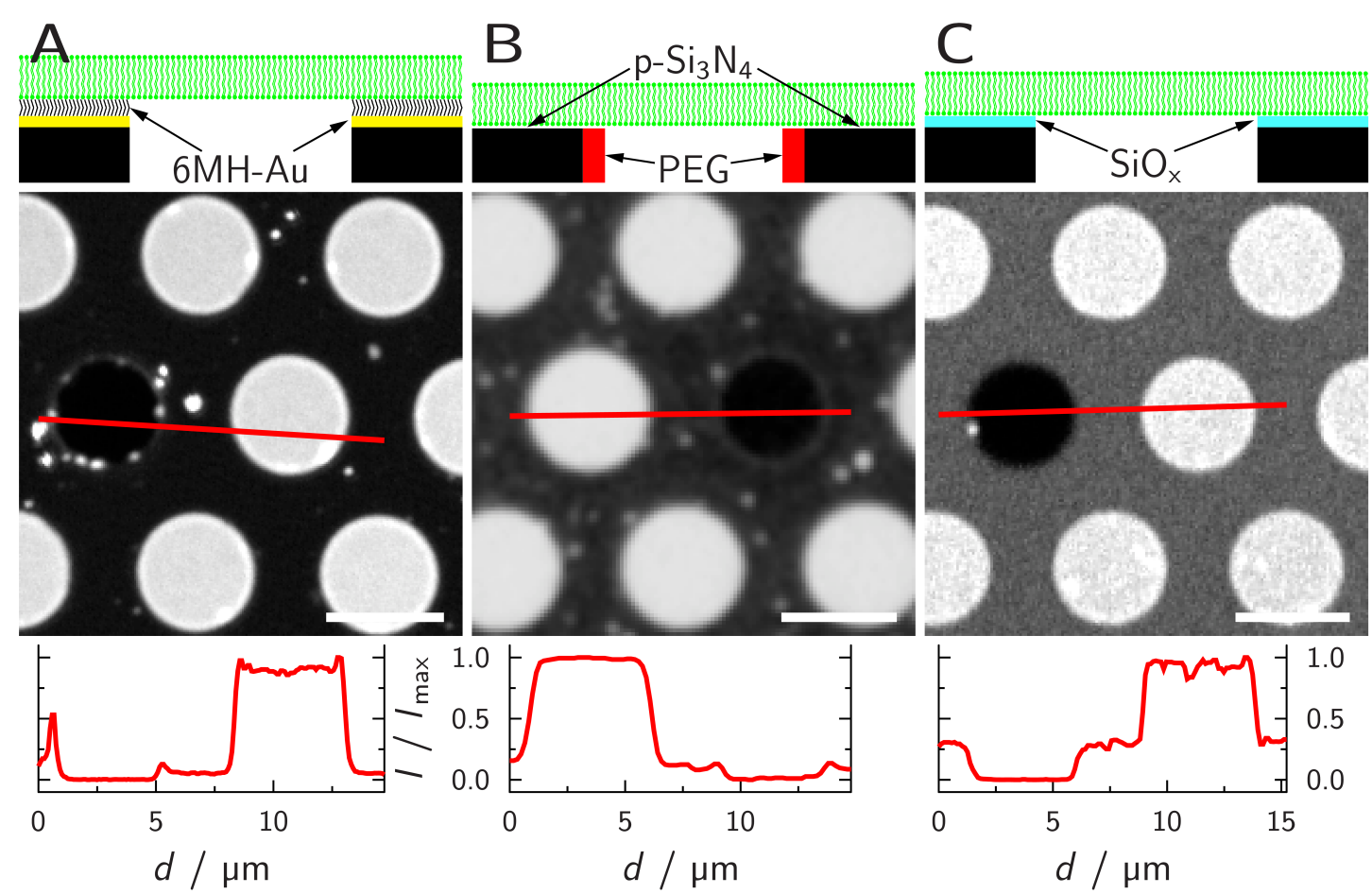

Figure 5.3.: Pore-spanning membranes (PSMs) on three different functionalizations A) $6 \mathrm{MH}-\mathrm{Au}, \mathrm{B}) \mathrm{p}-\mathrm{Si}_{3} \mathrm{~N}_{4}$ and $\mathrm{C}$ ) $\mathrm{SiO}_{\mathrm{x}}$. In the top row, schematic cross sections of the different functionalizations are shown. In the middle, fluorescence micrographs of PSMs composed of DOPC doped with ATTO488-DOPE on the different functionalizations are shown. From each fluorescent micrograph a profile line was taken at the red line and the intensity profile is plotted underneath. Scale bar $5 \mu \mathrm{m}$.

PSMs were generated by spreading of ATTO488-DOPE doped DOPC GUVs on the different functionalizations (figure 5.3 middle row). As expected, the different surface functionalizations influence the fluorescent information on s-PSM. While the f-PSMs are visible on the $6 \mathrm{MH}-\mathrm{Au}$ functionalization as bright round spots only minor to no fluorescence information on the rims can be gathered (figure $5.3 \mathrm{~A}$ ). Bright spots on the s-PSM were attributed to adhered, non-spread vesicles. The fluorescence intensity difference between a non-membrane spanned pore, representing the background fluorescence, s-PSM and f-PSM is shown in the cross section. The fluorescence intensity of the s-PSM is only $5 \%$ of the intensity measured on the f-PSM. This proves that no information on the s-PSM can be obtained with this system. Quenching of the fluorescence in the s-PSM is happening due to the short distance between membrane and gold surface. $217 \sqrt[219|222| 223]{ }$ Even if the $6 \mathrm{MH}$ builds a layer in between, this layer is only $\sim 1 \mathrm{~nm}$ thick 224 and the fluorophores are still in the quenching distance.

A fluorescent micrograph of a PSM with the $\mathrm{p}-\mathrm{Si}_{3} \mathrm{~N}_{4}$ functionalization is shown in figure 5.3 B. Again the f-PSM is detectable as bright round areas with $5 \mu \mathrm{m}$ in 
diameter. In contrast to the $6 \mathrm{MH}-\mathrm{Au}$ functionalization, the intensity of the s-PSM compared to the f-PSM intensity reaches nearly $15 \%$, as visualized by the intensity in the cross section (figure $5.3 \mathrm{~B}$ ). Comparable to the $6 \mathrm{MH}-\mathrm{Au}$ functionalization, adhered vesicles are visible as bright spots on the membrane.

The last analyzed functionalization strategy to the visualization of the PSM is the $\mathrm{SiO}_{\mathrm{x}}$ functionalization. A membrane patch was measured, as shown in figure $5.3 \mathrm{C}$. As described for the functionalizations $6 \mathrm{MH}-\mathrm{Au}$ and $\mathrm{p}-\mathrm{Si}_{3} \mathrm{~N}_{4}$, the f-PSM is detected as round areas of high fluorescence intensity. The s-PSM reaches a fluorescence intensity of $30 \%$ compared to the f-PSM, showing that this functionalization offers the best possibilities to gather fluorescence information on the s-PSM. A silicon dioxide surface has ideally no quenching, 217 but dependent on the evaporation techniques, full oxidation of the $\mathrm{SiO}_{\mathrm{x}}$ surface was not possible. 225. $\mathrm{SiO}_{\mathrm{x}}$ as a semiconductor still has distance dependent quenching properties, $226 \sqrt[228]{2}$ but the highest amount of visible fluorescence on the pore rims compared to the other analyzed functionalizations.

$\mathrm{SiO}_{\mathrm{x}}$ functionalized surfaces were used to study the phase separation on PSMs, especially on s-PSM (figure 5.4 and 5.5). Phase-separated GUVs composed of DOPC/ $\mathrm{SM}_{\text {porc }} / \mathrm{Chol} /$ porcine erythrocytes globotriaosyl ceramide $\left(\mathrm{Gb}_{3 \text { porc }}\right) /$ BODIPY-Chol/ TexasRed-DHPE (39.5:35:19.5:5:0.5:0.5) were spread at room temperature to generate phase-separated PSMs (chapter 3.2.3). In figure 5.4 A and $\mathrm{C}$ the fluorescence

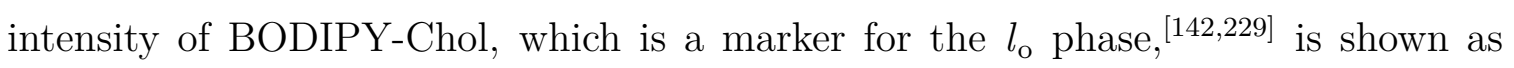
green color while in red the $l_{\mathrm{d}}$ phase is visualized by TexasRed-DHPE (figure $5.4 \mathrm{~B}$ and C). $139[140$ An intensity profile line of the corrected and normalized fluorescence intensities $\left(I / I_{\max }\right)$ is extracted which includes different f-PSMs (figure 5.4, white). Some f-PSMs are completely composed of $l_{\mathrm{o}}$ phase (green, 0-11 $\mu \mathrm{m}$ ), some completely of $l_{\mathrm{d}}$ phase (red, 14.5-16 $\mu \mathrm{m}$ ) and some consists of a small $l_{\mathrm{o}}$ phase surrounded by the $l_{\mathrm{d}}$ phase $(11-14.5 \mu \mathrm{m}$, figure 5.4). The line scans (yellow lines in figure 5.4) derived from the s-PSMs show an area mainly enriched by $l_{\mathrm{o}}$ phase in BODIPY-Chol, simultaneously excluding the $l_{\mathrm{d}}$ phase marker TexasRed-DHPE leading to $I / I_{\max }=0$ at $\mathrm{d}=2-6 \mu \mathrm{m}$. The $l_{\mathrm{o}}$ phase in the s-PSM is indicated within the white surrounded region in the fluorescence micrographs.

The merged fluorescence image (figure 5.4 C) and profile lines from the two fluorescence intensities BODIPY-Chol (green) and TexasRed-DHPE (red) prove the complete phase separation of $l_{\mathrm{o}}$ and $l_{\mathrm{d}}$ phases in PSMs (figure 5.4 $\mathrm{C}$ ). When BODIPYChol gives a high signal in either image or profile line, no signal from TexasRed-DHPE is detectable and vice versa.

Comparable phase-separated f-PSMs were also described by Schütte et al. 2230 They used the $6 \mathrm{MH}-\mathrm{Au}$ functionalization and were only able to detect the different phase 

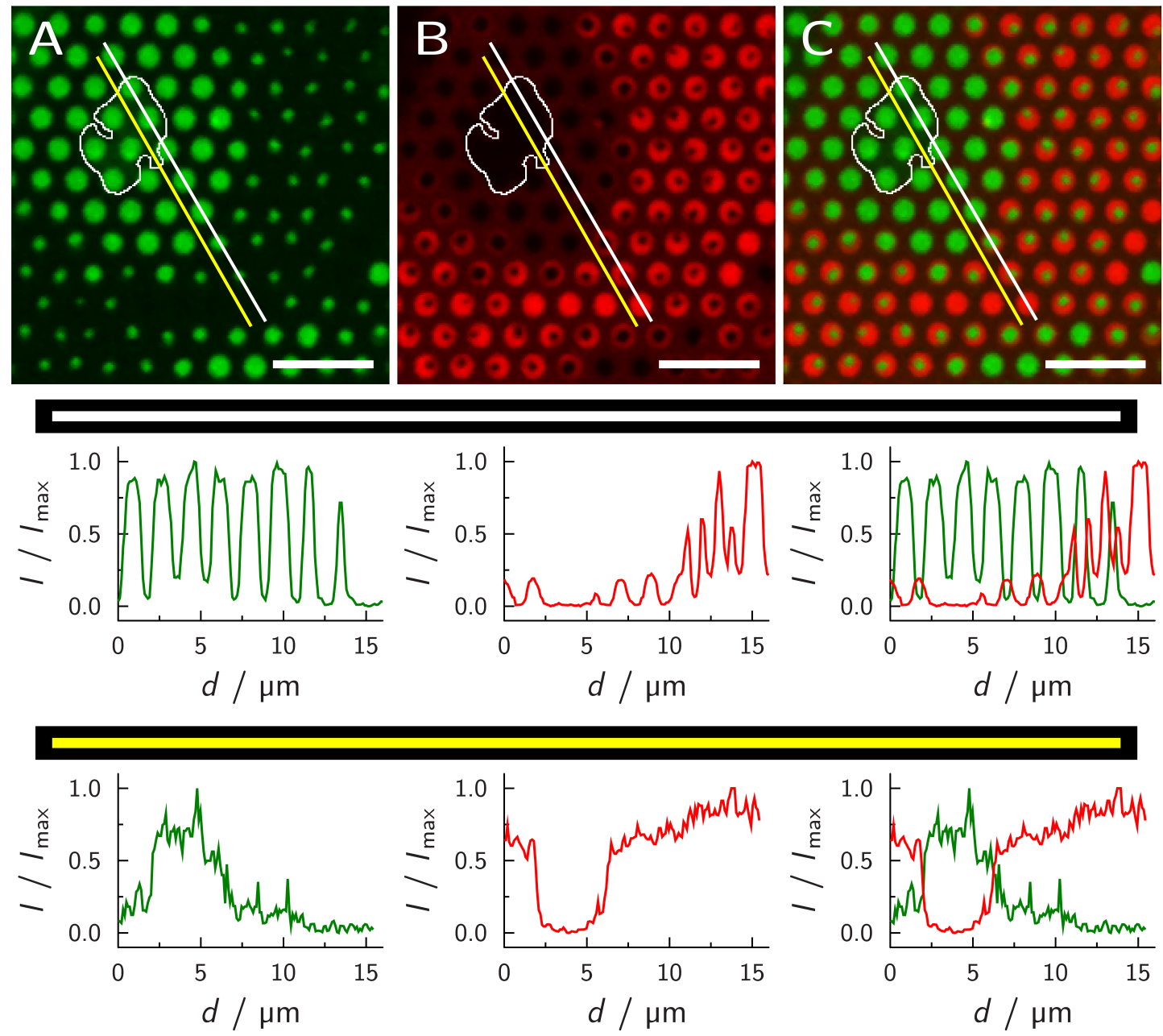

Figure 5.4.: Fluorescent images of phase-separated pore-spanning membrane (PSM) composed of DOPC/SM $\mathrm{SM}_{\text {porc }} / \mathrm{Chol} / \mathrm{Gb}_{3 \text { porc }} /$ BODIPY-Chol/TexasRed-DHPE (39.5:35:19.5: $5: 0.5: 0.5)$. Fluorescence of BODIPY-Chol, labeling the liquid ordered $\left(l_{\mathrm{o}}\right)$ phase is shown in green $(\mathrm{A}, \mathrm{C})$ and the fluorescence of TexasRed-DHPE, labeling the liquid disordered $\left(l_{\mathrm{d}}\right)$ phase is shown in red $(\mathrm{B}, \mathrm{C})$. In $\mathrm{C}$, a merged fluorescent image is shown to better visualize the fluorescence intensity differences of both channels. The corresponding yellow lines show the fluorescence intensity profiles of the solid supported pore-spanning membranes (s-PSMs) for the green and red channels, and the white line shows the fluorescence intensity profiles of the freestanding pore-spanning membranes (f-PSMs) for the green and red channels. The white area in the fluorescence images highlights the $l_{\mathrm{o}}$ phase in the s-PSM. Scale bar 5 um. 
separations in the f-PSM which are: f-PSM was full in $l_{\mathrm{d}}$ phase or $l_{\mathrm{o}}$ phase as well as phase-separated f-PSM. The use of $\mathrm{SiO}_{\mathrm{x}}$ extended the information to the description of phase separation in the s-PSM. Phase separation in PSMs was also measured by Sumitomo et al..231 They visualized only phase separation in the s-PSM and the $\mathrm{f}-\mathrm{PSM}$ in the $l_{\mathrm{d}}$ phase.

To investigate the possibilities to detect protein binding to PSMs, GUVs composed of DOPC/SM $\mathrm{Sm}_{\text {porc }} / \mathrm{Chol} / \mathrm{Gb}_{3 \text { porc }} /$ ATTO488-DPPE (39.9:35:20:5:0.1) were spread, generating PSMs on the $\mathrm{SiO}_{\mathrm{x}}$ functionalized porous substrates. The PSMs were incubated with $300 \mathrm{~nm}$ Cy3 labeled B-subunit of shiga toxin (STxB), which is expected to bind $\mathrm{Gb}_{3}$. An exemplary image of these PSMs and the bound proteins is shown in figure 5.5. The fluorescence intensity of the $l_{\mathrm{d}}$ phase marker, ATTO488-DPPE, 140] is shown in green (figure 5.5 A and C). Interestingly, fluorescence intensity from the $l_{\mathrm{d}}$ phase marker in the f-PSM is only visible near to the border of the f-PSMs. This is analyzed in more detail using intensity profile lines taken from different f-PSMs (figure 5.5 white line). In these profile lines, the intensity is highest at the border of the f-PSM and decreases in the middle of the f-PSM as well as on the s-PSM. While a reduction of fluorescence intensity from f-PSM to s-PSM is expected on the $\mathrm{SiO}_{\mathrm{x}}$ quenching effect described earlier, the decrease within the f-PSM seems to gather the $l_{\mathrm{d}}$ phase on the outer borders of the f-PSM while the middle of the pores comprises of $l_{\mathrm{o}}$ phase. $l_{\mathrm{d}}$ phase fluorescence intensity on the s-PSM is very heterogeneous which is shown in the profile line (figure 5.5 yellow line), small areas of low intensity give a strong hint to the existence of $l_{\mathrm{o}}$ phase domains on the s-PSM.

The fluorescence intensity of $\mathrm{Cy} 3$ labeled STxB is shown in red (figure 5.5 B and $\mathrm{C})$. The bright round areas in the fluorescence micrograph shows that the STxB binds in the f-PSM (figure $5.5 \mathrm{~B}$ and $\mathrm{C}$ ). This is also seen in the white profile line (figure $5.5 \mathrm{~B}$ and $\mathrm{C}$ ). The merged image of the two channels (figure 5.5 C) shows one spot without any intensity of both channels, this is a non-membrane spanned pore and also detectable in the range from 3.9 to $4.8 \mu \mathrm{m}$ in the f-PSM profile line (figure $5.5 \mathrm{C}$, white). Protein intensity is not co-localized with the $l_{\mathrm{d}}$ phase in the s-PSM, which is shown in the profile line (figure 5.5 C, yellow) where the fluorescence of the $l_{\mathrm{d}}$ phase marker decreases from 0.8 to $1.3 \mu \mathrm{m}$ and from 2.8 to $3.2 \mu \mathrm{m}$ and the STxB fluorescence was detectable.

The analysis of STxB Cy3 on phase-separated PSMs showed that the STxB Gb 3 binding takes place to the $l_{\mathrm{o}}$ phase in the f-PSM and s-PSM. This was also observed in GUVs 54 and on solid supported membranes (SSMs). 162 The $l_{\mathrm{o}} / l_{\mathrm{d}}$ phase separation with no incubation of STxB shows large areas of $l_{\mathrm{d}}$ phases in the s-PSM (figure 5.4). The incubation of STxB on such a system changes the morphology of the $l_{\mathrm{d}}$ phase 

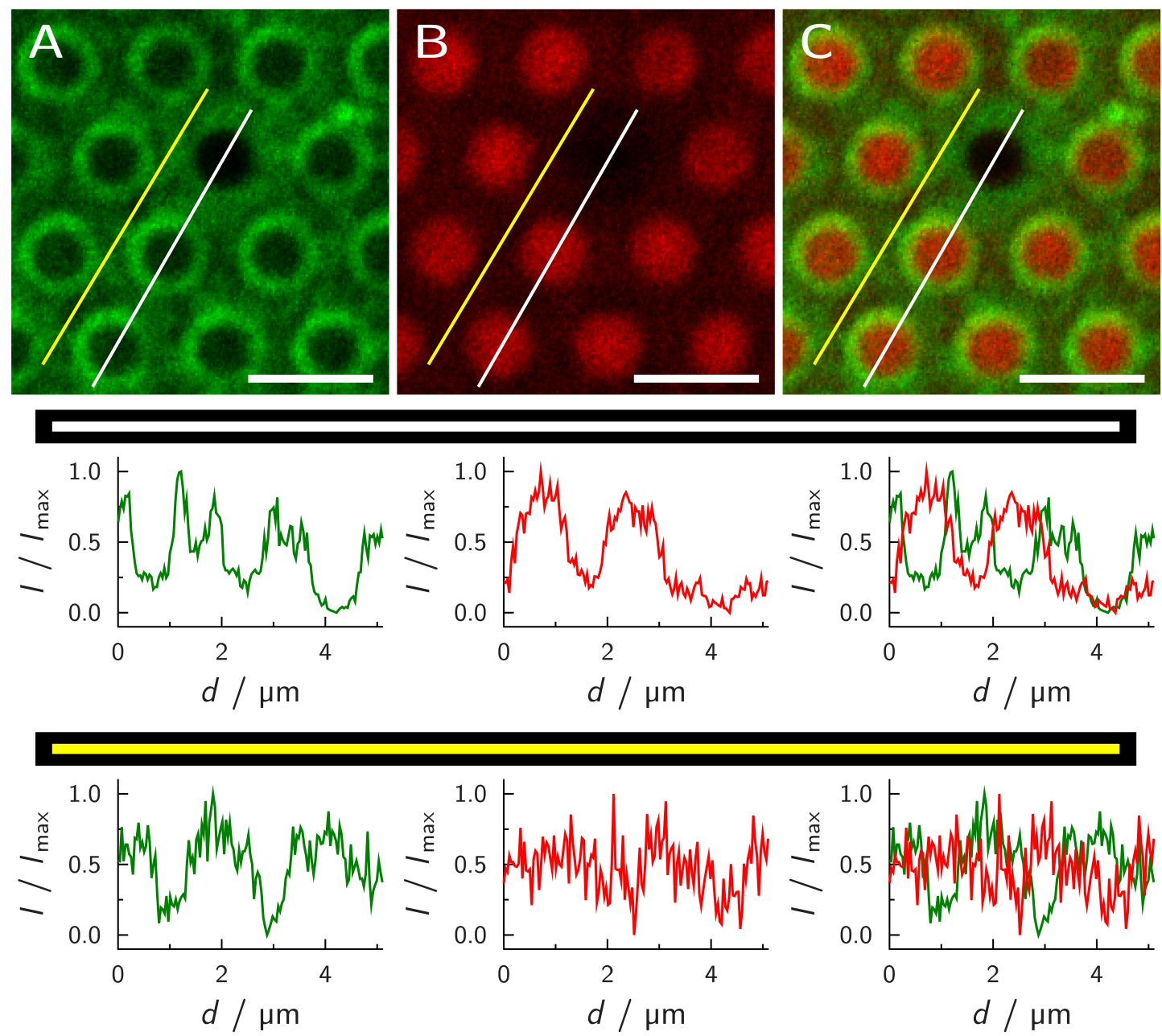

Figure 5.5.: Fluorescence images of phase-separated pore-spanning membrane (PSM) composed of DOPC/SM $\mathrm{Sorc}_{\text {p }} / \mathrm{Chol} / \mathrm{Gb}_{3 \text { porc }} / \mathrm{ATTO} 488$-DPPE (39.9:35:20:5:0.1) incubated with Cy3 labeled STxB (300 mm, 1 h). Fluorescence of ATTO488-DPPE, labeling the liquid disordered $\left(l_{\mathrm{d}}\right)$ phase is shown in green $(\mathrm{A}, \mathrm{C})$ and the fluorescence of Cy3 STxB is shown in red $(\mathrm{B}, \mathrm{C})$. Corresponding intensity profiles along the yellow line (solid supported porespanning membrane (s-PSM)) and along the white line (freestanding pore-spanning membrane (f-PSM)) are plotted below. Scale bar $2 \mu \mathrm{m}$. 
on the s-PSM. The $l_{\mathrm{d}}$ phase on the s-PSM was separated by small $l_{\mathrm{o}}$ domains. This can be a hint that the $\mathrm{STxB}$ recruits the $\mathrm{Gb}_{3}$ into the $l_{\mathrm{o}}$ phase. $55 \mid 162$

In this section three different functionalization strategies were analyzed. The possibility to generate PSMs and to detect s-PSM were investigated. The detection of membrane fluorophores in s-PSM is not possible with the 6MH-Au functionalization, because gold quenches the fluorophore. The fluorescence intensity of s-PSM on $\mathrm{p}-\mathrm{Si}_{3} \mathrm{~N}_{4}$ functionalized substrates is high enough to distinguish between s-PSM and background. The $\mathrm{SiO}_{\mathrm{x}}$ has the best signal-to-noise ratio of s-PSM fluorescence intensity. This enabled to detect phase-separated PSMs. The phase separation was not only visible in the f-PSM, but also in the s-PSM. As described in literature STx is expected to bind exclusively to the $l_{\text {o }}$ phase ${ }^{162}$ which was also found for the model of PSMs. STx binds to the s-PSMs as well as the f-PSMs in co-localization with the $l_{\mathrm{o}}$ phase in both regions.

\subsubsection{Membrane tension modulation of pore-spanning membranes (PSMs)}

Besides fluorescence intensity, PSMs membrane tension on different surface functionalization was analyzed. Tension was measured by AFM indentation experiments for all mentioned surface functionalizations. The generation of different functionalizations and the generation of PSMs were described in chapter 3.2 .2 and 3.2.3. Using correlative fluorescence microscopy, membrane patches were imaged and afterwards investigated by AFM. A topography image of a patch was done in quantitative imaging (QI) mode. In the center of the f-PSM, force-distance curves were measured. Following chapter 3.3 .3 , the tension of the membranes was derived.

According to Kuhlmann et al., membrane tension of the f-PSM is mainly determined by adhesion of the s-PSM. Lower adhesion is expected to lead to lower membrane tension. 214 Different functionalizations were investigated to check for the influence of chain length of chemisorbed mercaptoalcohols, plasma cleaned $\mathrm{Si}_{3} \mathrm{~N}_{4}$ and $\mathrm{SiO}$ evaporation to the surface hydrophobicity.

To evaluate the influence of chemisorbed thiols, especially the chain length difference of the mercaptoalcohols, GUVs composed of DOPC doped with ATTO488DPPE were spread on several functionalizations. The functionalizations are chemisorbed 2-mercapto-1-ethanol (2ME), 6MH, 8-mercapto-1-octanol (8MO), 11-mercapto1-undecanol (11MUD) and 16-mercapto-1-hexadecanol (16MHD) on gold. The length of the thiols is assumed to influence the packing density on the surface and therefore directly influences the hydrophilicity which could resulted in a higher adhesion. The 
results of the membrane tension measurements with the different mercaptoalcohols gold functionalizations are shown in figure 5.6 and table 5.1. As seen in figure 5.6. there is no direct correlation of length and tension, but different functionalization agents can influence the tension up to a factor of nearly 4. From substrate functionalization $2 \mathrm{ME}$ on gold $(2 \mathrm{ME}-\mathrm{Au})\left(1.6 \pm 1.1 \mathrm{mN} \mathrm{m}^{-1}\right)$ to $6 \mathrm{MH}-\mathrm{Au}\left(0.6 \pm 0.4 \mathrm{mN} \mathrm{m}^{-1}\right)$ membrane tension decreases but increases drastically using 8MO on gold (8MO$\mathrm{Au})\left(3.2 \pm 0.9 \mathrm{mN} \mathrm{m}^{-1}\right)$. Over 11MUD on gold (11MUD-Au) $\left(2.1 \pm 1.4 \mathrm{mN} \mathrm{m}^{-1}\right)$ to $16 \mathrm{MHD}$ on gold (16MHD-Au) $\left(1.0 \pm 0.4 \mathrm{mN} \mathrm{m}^{-1}\right)$ the tension is again decreased. The increase of membrane tension from $6 \mathrm{MH}-\mathrm{Au}$ to $8 \mathrm{MO}-\mathrm{Au}$ substrate functionalization interrupts the systematical decrease of membrane tension to the length of the mercaptoalcohols and the membrane tension has no dependency on the chain length from the mercaptoalcohols. The analysis of different membrane tensions was also done with a linear mixed-effects model (LMM), and the differences of membrane tension are not significant (table A.9.

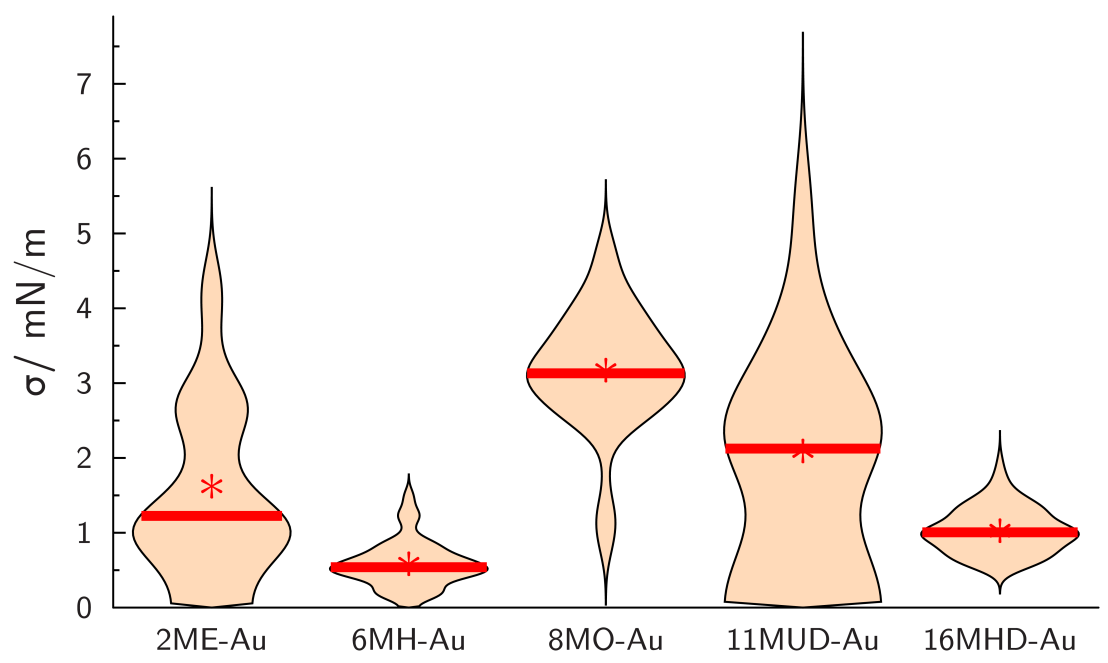

Figure 5.6.: The membrane tension $(\sigma)$ measured on gold mercaptoalcohol functionalizations. The lipid composition was DOPC/ATTO488-DPPE (99.5:0.5). The surface functionalizations were 2-mercapto-1-ethanol on gold (2ME-Au), 6-mercapto-1-hexanol on gold (6MH-Au), 8-mercapto-1-octanol on gold (8MO-Au), 11-mercapto-1-undecanol on gold (11MUD-Au) and 16-mercapto-1-hexadecanol on gold (16MHD-Au). The stroke indicates the median value in the violin plot and the cross the mean value.

If the chain length has no systematic influence on membrane tension, it seems that the surface density of the mercaptoalcohols is not chain length-dependent. The conformation of chemisorbed 2ME depends on thiol concentration. 232 A high solution concentration results in more trans conformations of the $\mathrm{Au}-\mathrm{S}-\mathrm{C}-\mathrm{C}$ chains which has a higher packing density. 232 Kudelski described that at concentrations higher than $1 \mathrm{~mm}$, the ratio of the conformations at the surface did not change which means 
Table 5.1.: The membrane tension $(\sigma)$ of the f-PSM composed of DOPC/ATTO488-DPPE (99.5:0.5) was measured on different functionalizations. The lipid composition of the $l_{\mathrm{o}}$ phase on $\mathrm{SiO}_{\mathrm{x}}$ functionalized substrate was $\mathrm{SM}_{\text {porc }} / \mathrm{Chol} /$ ATTO655-DOPE (59.5:39.5:1). The mean value and the standard derivation as the numbers of measurements $(N)$ were listed. The analyze method is described in chapter 3.3 .3 .

\begin{tabular}{|c|c|c|}
\hline functionalization & $\sigma\left[\mathrm{mN} \mathrm{m}^{-1}\right]$ & $N$ \\
\hline $2 \mathrm{ME}-\mathrm{Au}$ & $1.6 \pm 1.1$ & 251 \\
\hline $6 \mathrm{MH}-\mathrm{Au}$ & $0.6 \pm 0.4$ & 65 \\
\hline $8 \mathrm{MO}-\mathrm{Au}$ & $3.2 \pm 0.9$ & 98 \\
\hline 11MUD-Au & $2.1 \pm 1.4$ & 79 \\
\hline 16MHD-Au & $1.0 \pm 0.4$ & 353 \\
\hline $\mathrm{p}-\mathrm{Si}_{3} \mathrm{~N}_{4}$ & $1.6 \pm 0.5$ & 77 \\
\hline $\mathrm{SiO}_{x}$ & $10.5 \pm 4.3$ & 310 \\
\hline $\mathrm{SiO}_{\mathrm{x}} l_{\mathrm{o}}$ & $6.7 \pm 4.0$ & 59 \\
\hline
\end{tabular}

that the surface is packed to a maximum. $\frac{232}{2}$ In this thesis, the preparation of gold thiol functionalizations was done with a thiol concentration of $1 \mathrm{~mm}$. A high density packing was also found in electrochemical studies. Liu et al. analyzed the chemical conductance of gold to ferrocene by increasing the distance of the two materials. The distance was increased using chemisorped mercaptoalcohols with varying chain lengths from 6MH over 8MO and 11MUD to 12-mercapto-1-dodecanol (12MDD). 233 The electron transfer rate decreased with the elongation of the distance which proves that the mercaptoalcohol density on the gold surface is not dependent on the alkyl length of the mercaptoalcohol. Following this argument, the lateral tension should be the same for all mercaptoalcohols, since all are hydroxy-terminated which should result in equal surface properties. The deviations of the measurements are greater than the differences. It is not possible to find a reason for the differences of the membrane tension with the different mercaptoalcohol gold functionalizations. The high distribution can be an artefact of surface roughness or mobility of the functional groups.

Membrane tensions of different thiol gold functionalized substrates were measured and reported (summarized in table A.2). The membrane tension of DOPC membranes on 2ME-Au functionalization was measured of $1.018 \pm 0.014 \mathrm{mN} \mathrm{m}^{-1234}$ which is in good agreement with the values found in this work on $2 \mathrm{ME}-\mathrm{Au}$ with a DOPC bilayer of $1.6 \pm 1.1 \mathrm{mN} \mathrm{m}^{-1}$. The membrane tension with $6 \mathrm{MH}-\mathrm{Au}$ functionalization was measured by Kuhlmann et al. with a membrane composed of DPhPC $\left(1.4 \pm 0.1 \mathrm{mN} \mathrm{m}^{-1}\right) .214$ The range of the measured membrane tension from DOPC includes also the membrane tension value of $\mathrm{DPhPC}$. 
Furthermore, membrane tension of PSMs prepared on p- $\mathrm{Si}_{3} \mathrm{~N}_{4}$ and $\mathrm{SiO}_{\mathrm{x}}$ surfaces was determined. Measured membrane tensions from these functionalizations are listed in table 5.1 and are illustrated as violin plots in figure 5.7. The membrane tension of the f-PSM on the $\mathrm{p}-\mathrm{Si}_{3} \mathrm{~N}_{4}$ functionalized substrates $\left(1.6 \pm 0.5 \mathrm{mN} \mathrm{m}^{-1}\right)$ was in the same range as that with $2 \mathrm{ME}-\mathrm{Au}$ functionalization $\left(1.6 \pm 1.1 \mathrm{mN} \mathrm{m}^{-1}\right)$. The membrane tension with $\mathrm{SiO}_{\mathrm{x}}$ functionalization is more than three times higher than the highest and 18 times higher than the lowest tension found on chemisorped thiol functionalized substrates.

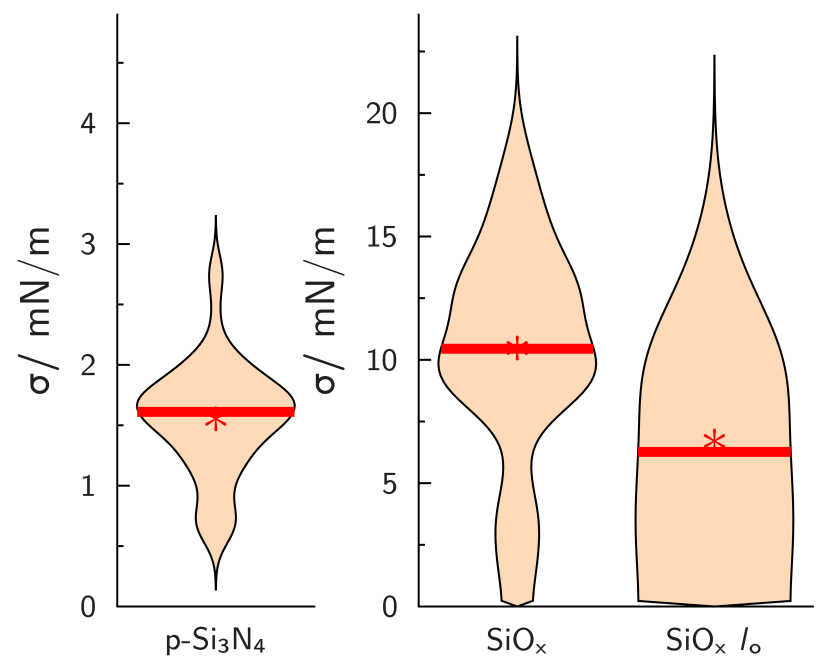

Figure 5.7.: The membrane tension $(\sigma)$ measured on gold mercaptoalcohol functionalizations. The lipid composition was DOPC/membrane marker (99.5:0.5) and for the $l_{\mathrm{o}}$ phase measurement $\mathrm{SM}_{\text {porc }} / \mathrm{Chol} /$ membrane marker (59.5:39.5:1). The surface functionalizations were plasma cleaned silicon nitride $\left(\mathrm{p}-\mathrm{Si}_{3} \mathrm{~N}_{4}\right)$ and silicon oxide $\left(\mathrm{SiO}_{\mathrm{x}}\right)$. The stroke indicates the median value in the violin plot and the cross the mean value.

Mey et al. used a porous glass and measured the membrane tension of it.235 The membrane tension was $0.15 \pm 0.05 \mathrm{mN} \mathrm{m}^{-1}$ with a DPhPC membrane. 235. This value is 70 times smaller than the membrane tension with the $\mathrm{SiO}_{\mathrm{x}}$ functionalization and the membrane lipid DOPC $\left(10.5 \pm 4.3 \mathrm{mN} \mathrm{m}^{-1}\right)$ and it is 10 times smaller than the membrane tension with the $\mathrm{p}-\mathrm{Si}_{3} \mathrm{~N}_{4}$ functionalization $\left(1.6 \pm 0.5 \mathrm{mN} \mathrm{m}^{-1}\right)$. It was concluded that the surface interaction of the $\mathrm{SiO}_{\mathrm{x}}$ and $\mathrm{p}-\mathrm{Si}_{3} \mathrm{~N}_{4}$ functionalizations to the s-PSM are stronger as the surface interaction in the study measured by Mey et al..235

As the membrane rupture tension of DOPC is at $\sim 10 \mathrm{mN} \mathrm{m}^{-1}, 236 \mid 237$ the f-PSMs on $\mathrm{SiO}_{\mathrm{x}}$ functionalized substrates were assumed to be in a critical state due to huge lateral tension. However, during the measurements, the f-PSMs were stable. The incorporation of Chol is known to stabilize the membrane, 119 as it was shown that the rupture tension increases to $\sim 19 \mathrm{mN} \mathrm{m}^{-1}$ for membranes containing $50 \mathrm{~mol} \%$ 
Chol.237 The measurement of phase-separated membranes with the lipid composition of DOPC/sphingomyelin (SM)/Chol therefore were expected to be less prone to rupture during the experiments compared to pure DOPC membranes.

The $\mathrm{SiO}_{\mathrm{x}}$ functionalization has the advantage that the phase separation of $l_{\mathrm{o}} / l_{\mathrm{d}}$ phase-separated membranes is visible in the f-PSM and s-PSM (figure 5.4). The interplay of tension and lipid composition is of great interest, therefore membrane tension of the two phases were also measured on $\mathrm{SiO}_{\mathrm{x}}$ functionalized substrates. The two different membrane phases were modeled by different membrane compositions. DOPC/membrane marker (99.5:0.5) was used to mimic the $l_{\mathrm{d}}$ phase and $\mathrm{SM}_{\text {porc }} /$ Chol/ATTO655-DOPE (59.5:39.5:1) for the $l_{\mathrm{o}}$ phase.238 The $l_{\mathrm{d}}$ phase had a membrane tension of $10.5 \pm 4.3 \mathrm{mN} \mathrm{m}^{-1}$ and the $l_{\mathrm{o}}$ phase $6.7 \pm 4.0 \mathrm{mN} \mathrm{m}^{-1}$ (figure 5.7 . table 5.1. The membrane tension of the $l_{\mathrm{d}}$ and $l_{\mathrm{o}}$ phase are not distinct within the errors of the measurement, as also seen in the statistical analysis (table A.9.

The $l_{\mathrm{o}}$ phase, high ordered structure, is expected to show higher membrane tension on $\mathrm{SiO}_{\mathrm{x}}$ functionalized substrates compared to $l_{\mathrm{d}}$ phase, low ordered structure, because membranes of DOPC PSMs shows lower membrane tensions compared to membranes with a higher ordering like DOPC/Chol.119|234 Such an effect was also measured with POPC membranes (table A.2. 239

The deviation of the expected result to the obtained results in this work can be explained taking the surface geometry and membrane surface interaction into account. The strength of the surface membrane interaction is not only a factor of surface hydrophobicity, but also roughness, 240] as known from wetting phenomena. Different studies were done with $l_{\mathrm{d}}$ and $l_{\mathrm{o}}$ phases on curved surfaces. In all studies, the $l_{\mathrm{d}}$

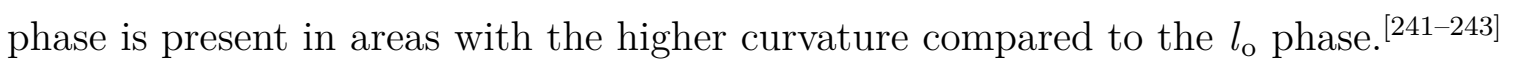
The $l_{\mathrm{o}}$ phase is associated with less curved areas, because it has a higher bending stiffness as the $l_{\mathrm{d}}$ phase and prefers flat surfaces. 243.254

The surface roughness of $\mathrm{SiO}_{\mathrm{x}}$ functionalized substrates was determined to be $0.49 \pm 0.03 \mathrm{~nm} .[148$ The differences of the expected and measured membrane tensions on $\mathrm{SiO}_{\mathrm{x}}$ functionalized substrates with $l_{\mathrm{o}}$ and $l_{\mathrm{d}}$ mimicking membranes can be explained by surface interactions. The $l_{\mathrm{o}}$ mimicking membrane has a lower adhesion, as it is not able to follow surface topography due to high bending energies needed to do so, while $l_{\mathrm{d}}$ membranes exhibit a low bending energy and therefore gain higher adhesional energies. The measured membrane tensions on $\mathrm{SiO}_{\mathrm{x}}$ functionalization are too widely spread to confirm or refute this assumption.

A comparison of membrane tension from different surface functionalizations shows, that membrane tension is affected by functionalization. Gold mercaptoalcohol and p- $\mathrm{Si}_{3} \mathrm{~N}_{4}$ have nearly the same membrane tensions, only $\mathrm{SiO}_{\mathrm{x}}$ has higher membrane 
tension by a factor of 7 ; this is close to the rupture tension of DOPC. Surface roughness can have an influence on membrane tension. If membrane is stiffer than the surface interaction can decrease, and the membrane tension also decreases. The physiological cell membrane tension of 0.01 to $0.15 \mathrm{mN} \mathrm{m}^{-1215216}$ could not be be reached with the different functionalizations. The membrane tension in f-PSMs is up to 70 times higher as the overall membrane tension of cells. Despite this big difference, the model system PSM is still more cell-similar than many other model systems, due to the different adhesion areas.

\subsubsection{Diffusion in pore-spanning membranes (PSMs) Interferometric scattering microscopy (iSCAT)}

As lipid rafts are transient lipid containing structures, diffusion of lipids is a major parameter to study the existence and properties of lipid rafts. The lipid diffusion is a property of each biomimetic membrane system influenced by artificial factors like membrane tension, contact to solid supports, and lipid composition. Therefore, for every surface functionalization, also lipid diffusion was characterized. Since fluorescence-based techniques are not available to quantify the diffusion coefficient of the lipids in the s-PSM, for the mercaptoalcohol gold functionalization due to quenching, interferometric scattering microscopy (iSCAT) measurements were used (chapter 3.3.2). iSCAT is a single particle tracking (SPT) method in which a gold nano particle (GNP) is coupled via streptavidin biotinyl to 1,2-dioleoyl-sn-glycero3-phosphoethanolamine-N-cap-biotinyl (cb-DOPE) and its movement is recorded using interference. A time series was recorded for $5 \mathrm{~s}$ with a temporal resolution of $1 \mathrm{kHz}$. From the trajectories of the GNP, the diffusion coefficient was determined using covariance-based estimation. 154255 This measurement was conducted with two different functionalized surfaces, $6 \mathrm{MH}-\mathrm{Au}$ and $\mathrm{p}-\mathrm{Si}_{3} \mathrm{~N}_{4}$.

Three different GNP paths were observed during iSCAT measurements. First, the GNP diffusion was only detected on the s-PSM (figure 5.8 A). Second, the GNP diffused on both membrane areas, the s-PSM and f-PSM (figure $5.8 \mathrm{~B}$ ). Third, the GNP diffusion was only detectable on the f-PSM (figure $5.8 \mathrm{C}$ ).

The GNP diffused freely on both, the f-PSM and s-PSM. The diffusion coefficient from the GNP was determined separately on both parts of the PSM system and is summarized in table 5.2 and figure 5.9. Comparing the diffusion on the f-PSM for both functionalizations (figure 5.9 A), the motion of the GNP on f-PSMs prepared on $\mathrm{p}-\mathrm{Si}_{3} \mathrm{~N}_{4}$ functionalized substrates was faster $\left(2.6 \pm 1.0 \mu \mathrm{m}^{2} \mathrm{~s}^{-1}\right)$ than on $6 \mathrm{MH}-\mathrm{Au}$ functionalized substrates $\left(1.8 \pm 0.7 \mathrm{\mu m}^{2} \mathrm{~s}^{-1}\right.$, figure 5.9 A, table 5.2 . 

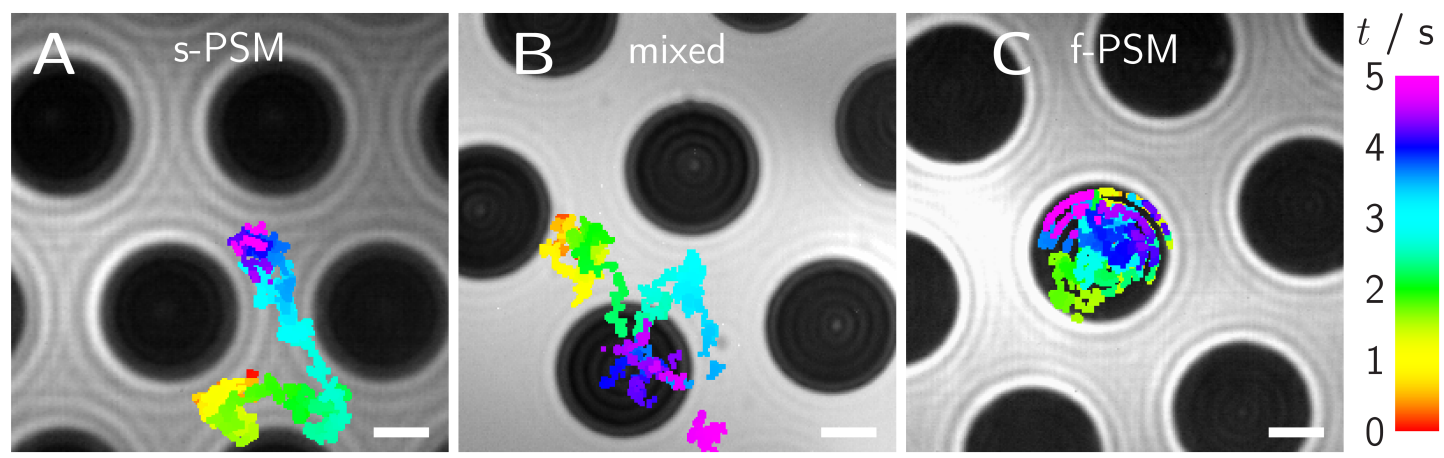

Figure 5.8.: Raw iSCAT images from videos recorded at $1 \mathrm{kHz}$ for $5 \mathrm{~s}$ overlaid with trajectories of $40 \mathrm{~nm}$ GNPs attached to cb-DOPE lipids diffusing in a DOPC membrane on a p-Si $\mathrm{N}_{4}$ functionalized substrate. A) Trajectory of a GNP on the s-PSM. B) Trajectory of a GNP moving on the s-PSM, crossing over to the f-PSM and return to the s-PSM. C) Trajectory of a GNP trapped in the f-PSM. The color code shows the time evolution in each case. Scale bar $2 \mu \mathrm{m}$

Table 5.2.: The diffusion coefficient $(D)$ were measured on different functionalized substrates to generate PSMs composed of DOPC/cb-DOPE/ATTO532-DOPE (98.8:1: $0.2)$. The surface functionalizations were plasma cleaned silicon nitride $\left(\mathrm{p}-\mathrm{Si}_{3} \mathrm{~N}_{4}\right)$ and 6mercapto-1-hexanol on gold $(6 \mathrm{MH}-\mathrm{Au})$. The diffusion coefficient was measured with interferometric scattering microscopy (iSCAT) and from gold nano particles (GNPs), which are covered with streptavidin and connected via streptavidin biotinyl bond to the cb-DOPE in the membrane. The different freestanding pore-spanning membrane (f-PSM) and solid supported pore-spanning membrane (s-PSM) were analyzed separately. The mean values and the standard deviations were listed with the number of taken trajectories $(N)$.

\begin{tabular}{lcc} 
System / Surface & $D\left[\mathrm{~mm}^{2} \mathrm{~s}^{-1}\right]$ & $N$ \\
\hline $\mathrm{f}-\mathrm{PSM} / \mathrm{p}-\mathrm{Si}_{3} \mathrm{~N}_{4}$ & $2.6 \pm 1.0$ & 28 \\
$\mathrm{~s}-\mathrm{PSM} / \mathrm{p}-\mathrm{Si}_{3} \mathrm{~N}_{4}$ & $0.8 \pm 0.5$ & 745 \\
f-PSM / 6MH-Au & $1.8 \pm 0.7$ & 89 \\
s-PSM / 6MH-Au & $1.9 \pm 0.7$ & 279
\end{tabular}

The differences in f-PSM diffusion constants are explained by the difference in membrane tension of the two different functionalizations. The membrane tension of f-PSM with $6 \mathrm{MH}-\mathrm{Au}$ functionalized substrates is $0.6 \pm 0.4 \mathrm{mN} \mathrm{m}^{-1}$, which is a factor of 3 lower than found for the $\mathrm{p}-\mathrm{Si}_{3} \mathrm{~N}_{4}$ functionalized substrates $1.6 \pm 0.5 \mathrm{mN} \mathrm{m}^{-1}$. The diffusion constant increases from $6 \mathrm{MH}-\mathrm{Au}$ functionalized substrates with $1.8 \pm 0.5 \mathrm{\mu m}^{2} \mathrm{~s}^{-1}$ to $\mathrm{p}-\mathrm{Si}_{3} \mathrm{~N}_{4}$ functionalized substrates with $2.6 \pm 1.0 \mathrm{\mu m}^{2} \mathrm{~s}^{-1}$. This dependency of membrane tension on diffusion was also shown in molecular dynamics simulations (MDs) $256[257$ and was also measured using FCS on GUVs, using micro pipette aspiration to tune the GUV tension. 258 Interestingly, bulk methods like fluorescence recovery after photobleaching (FRAP) on GUVs and the micro pipette aspiration did not enable to measure the increase found in single lipid diffusion studies. 259 

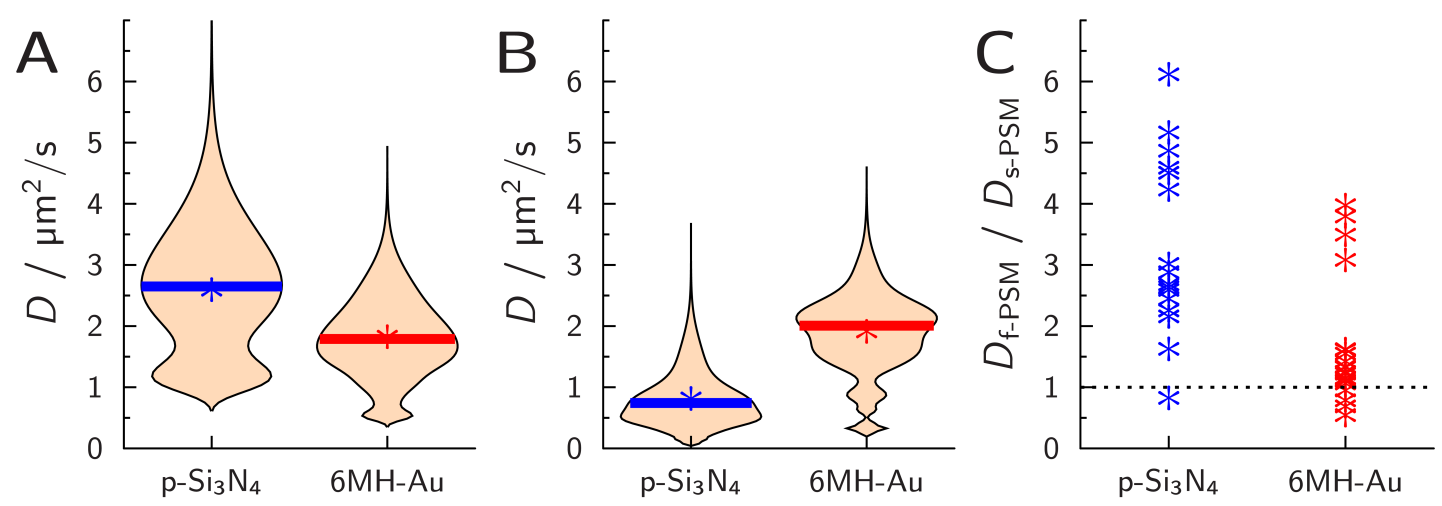

Figure 5.9.: The diffusion coefficient $(D)$ measured with interferometric scattering microscopy (iSCAT) on the pore-spanning membranes (PSMs) composed of DOPC/cbDOPE/ATTO532-DOPE (98.8:1:0.2). The substrates were functionalized with plasma cleaned silicon nitride $\left(\mathrm{p}-\mathrm{Si}_{3} \mathrm{~N}_{4}\right)$ or 6-mercapto-1-hexanol on gold $(6 \mathrm{MH}-\mathrm{Au})$. The diffusion coefficient of the freestanding pore-spanning membrane (f-PSM) (A) and solid supported pore-spanning membrane (s-PSM) (B) are compared to the different functionalizations. Some particles were observed in the f-PSM and s-PSM and from these particles the diffusion ratio $\left(D_{\mathrm{f}-\mathrm{PSM}} / D_{\mathrm{s}-\mathrm{PSM}}\right)$ were plotted in $\mathrm{C}\left(N\left(\mathrm{p}-\mathrm{Si}_{3} \mathrm{~N}_{4}\right)=16 ; N(6 \mathrm{MH}-\mathrm{Au})=21\right)$. The numbers and mean values are listed in table 5.2

The GNPs on the s-PSM with the $\mathrm{p}_{-} \mathrm{Si}_{3} \mathrm{~N}_{4}$ functionalization were slower than the GNPs on the s-PSM with the $6 \mathrm{MH}-\mathrm{Au}$ functionalization (figure $5.9 \mathrm{~B}$, table 5.2 ). The substrate membrane interaction decreases the lipid diffusion. The iSCAT measurements of DOPC membranes supported on glass and or in GUVs show the influence of the membrane support. Hsieh et al. measured the diffusion coefficient on SSM to $0.22 \mu \mathrm{m}^{2} \mathrm{~s}^{-1260}$ which has an high membrane adhesion, and Spindler et al. measured

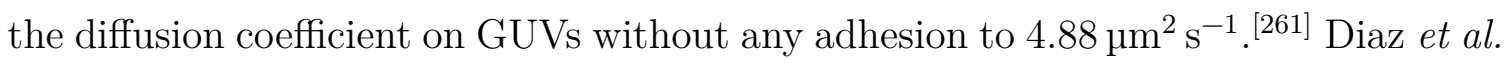
showed with protein diffusion directly on glass $\left(0.3 \pm 0.1 \mathrm{um}^{2} \mathrm{~s}^{-1}\right.$, high adhesion) or

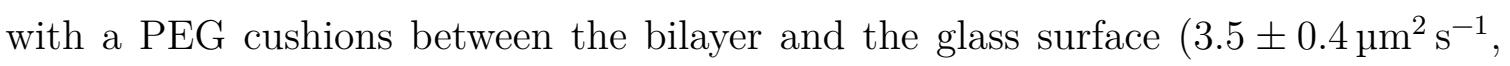
low adhesion) that different membrane surface adhesions had a comparable influence on the diffusion coefficient. 106 This indicates that the membrane substrate interaction was stronger when the substrate was functionalized with p- $\mathrm{Si}_{3} \mathrm{~N}_{4}$ compared to the $6 \mathrm{MH}-\mathrm{Au}$ functionalization. The stronger substrate membrane interaction was also shown by measurements of the membrane tension on these systems (table 5.1). High adhesion of the s-PSM is expected to result in a high lateral tension of the f-PSM and vice versa ( $\mathrm{p}-\mathrm{Si}_{3} \mathrm{~N}_{4}$ functionalization: $\sigma=1.6 \pm 0.5 \mathrm{mN} \mathrm{m}^{-1}$ and $D=0.8 \pm 0.5 \mathrm{\mu m}^{2} \mathrm{~s}^{-1} ; 6 \mathrm{MH}-\mathrm{Au}$ functionalization: $\sigma=0.6 \pm 0.4 \mathrm{mN} \mathrm{m}^{-1}$ and $D=$ $\left.1.9 \pm 0.7 \mu \mathrm{m}^{2} \mathrm{~s}^{-1}\right)$.

A direct comparison of the GNP diffusion connected to the f-PSM and s-PSM was done using particles which were observed on both regions in one experiment 
(figure $5.9 \mathrm{C})$. The resulting diffusion ratio $\left(D_{\mathrm{f}-\mathrm{PSM}} / D_{\mathrm{s}-\mathrm{PSM}}\right)$ describes the factor of how much faster the GNP is on the f-PSM compared to s-PSM. The diffusion ratio on the $\mathrm{p}-\mathrm{Si}_{3} \mathrm{~N}_{4}$ functionalized substrates showed different accumulations and ranged from 1 to 6 . In contrast to the strong difference between f-PSM and s-PSM, the diffusion ratio of GNPs from membranes prepared on $6 \mathrm{MH}-\mathrm{Au}$ functionalized surfaces is one. Indicating that the GNP diffusion constant of f-PSM and s-PSM is the same.

With iSCAT, only diffusion in the top leaflet is detectable, which might explain the diffusion constant ratio of one for the $6 \mathrm{MH}-\mathrm{Au}$ System, when interaction between membrane and surface is mainly influencing the bottom leaflet. A disadvantage of the iSCAT measurements were the unknown connection points from the GNP to the membrane. The GNP is coated with streptavidin which had four binding sides to biotin and two bindings each on the opposite side. So each streptavidin is able to bind two biotin labeled lipids and the GNP is coated with more than one streptavidin. In these results the GNP is able to bind several biotin labeled lipids. Liao et al. analyzed different sizes of GNP to evaluate the influence of the unknown GNP membrane binding points. 262 The smaller GNPs were faster than the greater ones (table A.1 which indicates an influence of the binding sites or size of the GNPs to the diffusion constant.

\section{Fluorescence correlation spectroscopy (FCS)}

To investigate the diffusion within both leaflets and ensure only single lipid diffusion is measured, Z-scan FCS measurements were performed (chapter 3.3.1). The measurements were done on $\mathrm{SiO}_{\mathrm{x}}$ functionalized substrates, because they had the advantage that $l_{\mathrm{o}} / l_{\mathrm{d}}$ phase-separated PSMs were detectable in the f-PSM and s-PSM (figure 5.4. To be able to distinguish between these phases with Z-scan FCS, different lipid composition were used to mimic the $l_{\mathrm{d}}$ and $l_{\mathrm{o}}$ phase. The $l_{\mathrm{d}}$ phase was mimicked with a DOPC/ATTO488-DOPE (99.5:0.5) membrane and the $l_{\mathrm{o}}$ phase was mimicked with a $\mathrm{SM}_{\text {porc }} / \mathrm{Chol} / \mathrm{ATTO} 655-\mathrm{DOPE}$ (59.5:39.5:1) membrane. The fluorophores for the Z-scan FCS measurements were added in low concentrations $\left(1 \times 10^{-3}-1 \times 10^{-5} \mathrm{~mol} \%\right)$. The fluorophores which were used to measure the diffusion in the $l_{\mathrm{d}}$ phase were ATTO655-DOPE or TexasRed-DHPE and for the $l_{\mathrm{o}}$ phase BODIPY-Chol or naphtho[2,3-a]pyrene (naphthopyrene). 140 The resulting diffusion coefficients are listed in table 5.3 and visualized in figure 5.10. The measurements demonstrate, that ATTO655-DOPE and TexasRed-DHPE had the same diffusion constants in the f-PSM (figure $5.10 \mathrm{~A}$ ). 

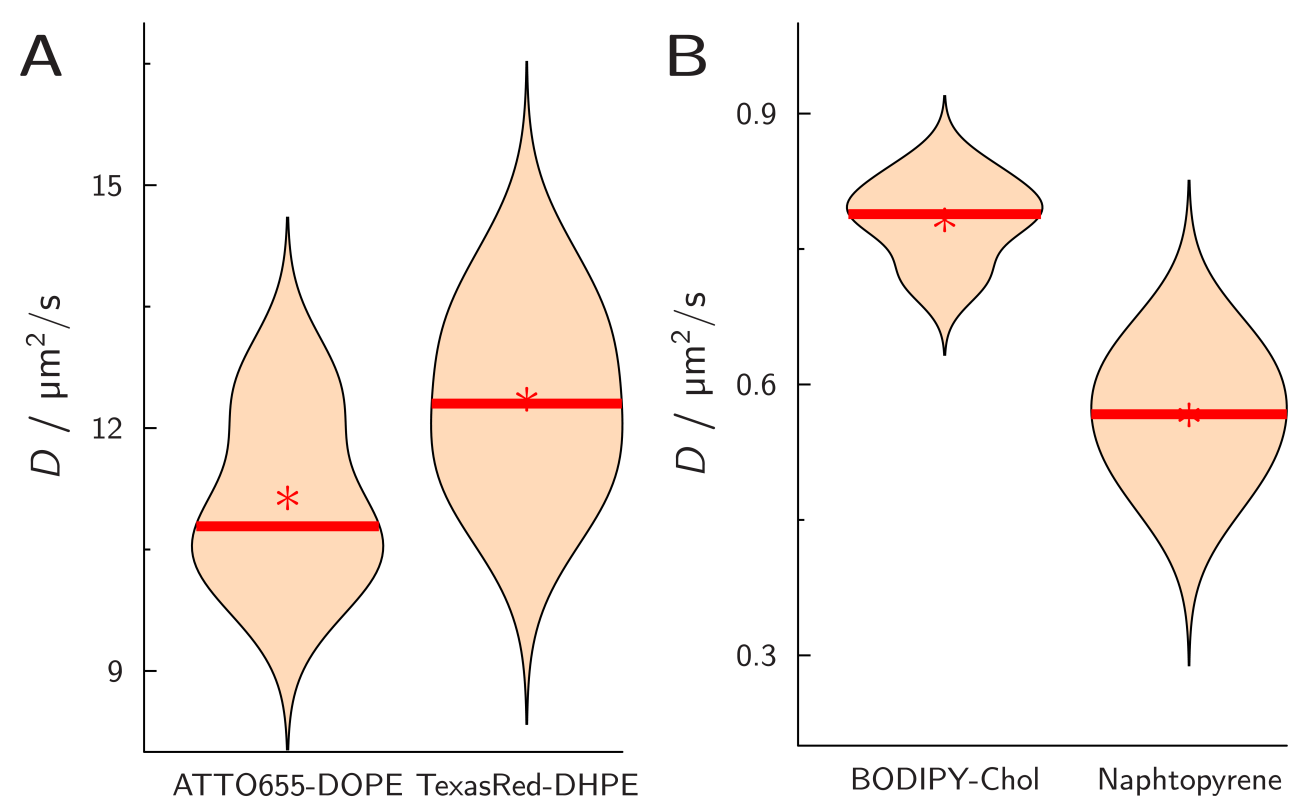

Figure 5.10.: The diffusion coefficient $(D)$ measured with Z-scan fluorescence correlation spectroscopy (Z-scan FCS) on the freestanding pore-spanning membrane (f-PSM). The substrates were functionalized with $\mathrm{SiO}_{\mathrm{x}}$. In A) the lipid composition of the membrane was DOPC/ATTO488-DOPE (99.5:0.5) doped with the Z-scan FCS fluorophores ATTO655DOPE or TexasRed-DHPE and in B) $\mathrm{SM}_{\text {porc }} / \mathrm{Chol} / \mathrm{ATTO} 655$-DOPE (59.5:39.5:1) doped with the Z-scan FCS fluorophores BODIPY-Chol or naphthopyrene.

The measurement of the diffusion constants in the f-PSM with $l_{\mathrm{o}}$ phase mimicking membrane was done with BODIPY-Chol and naphthopyrene (figure $5.10 \mathrm{~B}$ ). The diffusion of BODIPY-Chol $\left(0.78 \pm 0.05 \mu^{2} \mathrm{~s}^{-1}\right)$ was faster than the diffusion of naphthopyrene $\left(0.57 \pm 0.07 \mathrm{~mm}^{2} \mathrm{~s}^{-1}\right)$, but both diffusion coefficients were compared with the lipids in the $l_{\mathrm{d}}$ phase by a factor of 13 to 20 slower, which still allows for qualitative interpretations.

The diffusion coefficient of different phase-separated membranes were measured and reported in previous literature (table A.1). The diffusion coefficient of phaseseparated GUVs composed of DOPC/SM porc $/$ Chol $(2: 2: 1)$ was $6.1 \mu^{2} \mathrm{~s}^{-1}$ in the $l_{\mathrm{d}}$ phase which was slower by a factor of 20 in the $l_{\text {o phase }}\left(0.3 \mu \mathrm{m}^{2} \mathrm{~s}^{-1}\right)$ measured with Z-scan FCS.263 A second example with non-adhered membranes (GUVs) composed of DOPC/SM C $18: 0 /$ Chol (1:1:1) was measured with FCS. The diffusion was faster by a factor of 20 in the $l_{\mathrm{d}}$ phase compared to the diffusion in the $l_{\mathrm{o}}$ phase. $\left(5.15 \mathrm{\mu m}^{2} \mathrm{~s}^{-1}\right.$ $l_{\mathrm{d}}$ and $0.255 \mathrm{um}^{2} \mathrm{~s}^{-1} l_{\mathrm{o}}$ phase). 264 The difference in the diffusion coefficient of the $l_{\mathrm{o}}$ and $l_{\mathrm{d}}$ phase in the $\mathrm{f}-\mathrm{PSM}$ is in agreement with previous literature.

The diffusion coefficient of DOPC f-PSM mimicking the $l_{\mathrm{d}}$ phase $\left(\sim 11.8 \mu \mathrm{m}^{2} \mathrm{~s}^{-1}\right)$ is higher than the diffusion coefficient measured in DOPC GUVs. Different FCS measurements were reported in DOPC GUVs with diffusion coefficients from 6.3 to 
Table 5.3.: Diffusion coefficients $(D)$ were measured with Z-scan fluorescence correlation spectroscopy (Z-scan FCS) in the freestanding pore-spanning membrane (f-PSM) composed of DOPC/ATTO488-DOPE (99.5:0.5) for the liquid disordered $\left(l_{\mathrm{d}}\right)$ phase and $\mathrm{SM}_{\text {porc }} /$

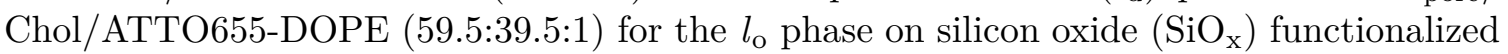
substrates. The different FCS fluorophores were added in $1 \times 10^{-3}$ to $1 \times 10^{-5} \mathrm{~mol} \%$. The mean values and the standard deviations were listed with the number of measured f-PSM trajectories $(N)$.

\begin{tabular}{cccc} 
Phase & Fluorophore & $D\left[\mathrm{\mu m}^{2} \mathrm{~s}^{-1}\right]$ & $N$ \\
\hline$l_{\mathrm{d}}$ & ATTO655-DOPE & $11.2 \pm 0.9$ & 3 \\
$l_{\mathrm{d}}$ & TexasRed-DHPE & $12.4 \pm 1.1$ & 6 \\
$l_{\mathrm{o}}$ & BODIPY-Chol & $0.78 \pm 0.05$ & 4 \\
$l_{\mathrm{o}}$ & naphthopyrene & $0.57 \pm 0.07$ & 6
\end{tabular}

$8.5 \mathrm{um}^{2} \mathrm{~s}^{-1}$ (table A.1. $170[264266$ However, as stated before this can be explained by the increased membrane tension of f-PSM compared to GUVs. 256 258 The membrane tension of GUVs can be assumed to be zero and the membrane tension of the f-PSM composed of DOPC on $\mathrm{SiO}_{\mathrm{x}}$ functionalized substrates was $10.5 \mathrm{mN} \mathrm{m}^{-1}$ (table 5.1 ).

The diffusion coefficient of the f-PSM composed of DOPC/POPE/POPS/Chol (5: 2:1:2) was measured by Schwenen et al. with FCS and 6MH-Au functionalized substrates, which were found to be $\sim 7.55 \mu^{2} \mathrm{~s}^{-1}$. 267. The diffusion coefficient found by Schwenen et al. is roughly lower by a factor of 1.5 than the diffusion coefficient measured on f-PSM composed of DOPC on $\mathrm{SiO}_{\mathrm{x}}$ functionalized substrates. As described in literature, Chol has an influence on the diffusion constant within the membranes. The increase of the Chol content from 20 to $66 \mathrm{~mol} \%$ in a DOPC GUVs reduced the diffusion coefficient by a factor of 2, which was measured with FCS. 264 Schwenen et al. used $20 \mathrm{~mol} \% \mathrm{Chol}$ which was expected to decrease lipid diffusion compared to the membrane systems used in this thesis.

In contrast to the literature, the comparison of FCS measurements of Schwenen et al. with the iSCAT measurements in the f-PSM on the 6MH-Au functionalized

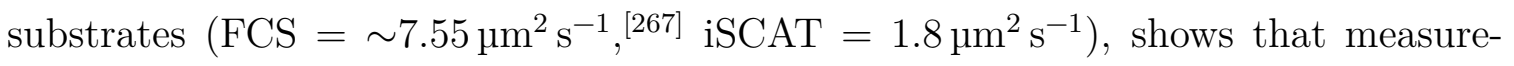
ments without Chol had a lower diffusion compared to measurements executed on Chol rich membranes in f-PSMs. The measurements were performed with two different techniques. The comparison of the two techniques on the same measurement system (DOPC GUVs), resulted in diffusion coefficients of $4.88 \mu \mathrm{m}^{2} \mathrm{~s}^{-1261}$ (iSCAT) and of 6.3 to $8.5 \mu^{2} \mathrm{~s}^{-1}$ (FCS, table A.1). 170|264 266. The difference of the techniques in GUVs can be sum up due to the effect of the size from the analyzed molecule. 262 While iSCAT only takes the upper leaflet of the lipid membrane in the f-PSM on a $6 \mathrm{MH}-\mathrm{Au}$ functionalized substrate into account, FCS considers both leaflets, which 
might induce the differences when comparing these techniques as the substrate functionalization also influences the bilayer coupling.

In this work the lipid diffusion was measured with two different techniques, iSCAT and FCS in PSMs. The diffusion coefficients depended on the functionalization of the substrate and the technique. iSCAT is a non-fluorescence technique and was used to measure the diffusion of the s-PSM and f-PSM. FCS is capable of distinguishing $l_{\mathrm{o}}$ and $l_{\mathrm{d}}$ phase within the f-PSM. The measured diffusion constant of the $l_{\mathrm{o}}$ phase was in the range of the lipid diffusion of cellular membranes (from 0.3 to $5.4 \mu^{2} \mathrm{~s}^{-1}$ ). $266|268| 271$ Despite the still too large membrane tension, it matches this model appropriate to study diffusion control mechanisms for processes involved in raft formation.

\subsection{Phase separation in pore-spanning membranes (PSMs)}

Utilizing $\mathrm{SiO}_{\mathrm{x}}$ functionalized substrates, phase separation behavior in dependency of Chol content and temperature was studied on f-PSMs and s-PSMs. Therefore, GUVs composed of DOPC/SM $\mathrm{Sm}_{\text {porc }} /$ Chol were spread on these substrates to generate the PSMs. The Chol content was varied between 0 to $50 \mathrm{~mol} \%$ while keeping DOPC and $\mathrm{SM}_{\text {porc }}$ content all the time at a 1:1 ratio. To measure phase separation

behavior of $l_{\mathrm{d}}$ and $l_{\mathrm{o}}$ phases, TexasRed-DHPE was used as a $l_{\mathrm{d}}$ phase marker $139 \mid 140$ and BODIPY-Chol as a $l_{\mathrm{o}}$ phase marker. $140 \mid 142$ The temperature was varied between 25 and $55^{\circ} \mathrm{C}$. The measurement of each fluorescence image was done at a certain temperature. Fluorescence images were recorded only once the temperature reached a stable plateau.

Figure 5.11 shows typical fluorescence micrographs of PSMs with BODIPY-Chol (green) and TexasRed-DHPE (red) being in a phase-separated state $\left(T=25^{\circ} \mathrm{C}\right)$ and in a non-phase-separated state $\left(T=55^{\circ} \mathrm{C}\right)$. To calculate absolute intensities, an uncovered pore was indicated with an $\mathrm{x}$ in each fluorescence image which was used for background intensity calculations. Two different lipid compositions are shown, i.e., DOPC/SM porc $_{\text {Chol (41.5:41.5:17) (A) and DOPC/SM }}$ porc $/$ Chol (47:47:6) (B). At $55^{\circ} \mathrm{C}$, the non-phase-separated state, the fluorophores show red and green fluorescence co-localized in f-PSM and s-PSM (figure 5.11 A and B, T=55 ${ }^{\circ} \mathrm{C}$ ). As discussed before, s-PSM intensity was diminished compared to f-PSM intensity due to quenching effects. 148 Co-localization of both lipid phase markers proves that only one phase exists and both fluorophores are soluble in the $l_{\mathrm{d}}$ phase as well as the temperature was above the miscible temperature. That means that the membrane 
was existent in the $l_{\mathrm{d}}$ phase. At $25^{\circ} \mathrm{C}$, a phase-separated state of the PSM was found (figure 5.11 A and B, $T=25^{\circ} \mathrm{C}$ ). While the s-PSMs showed a homogeneous TexasRed-DHPE fluorescence, no TexasRed-DHPE fluorescence intensity was measured in the f-PSMs.
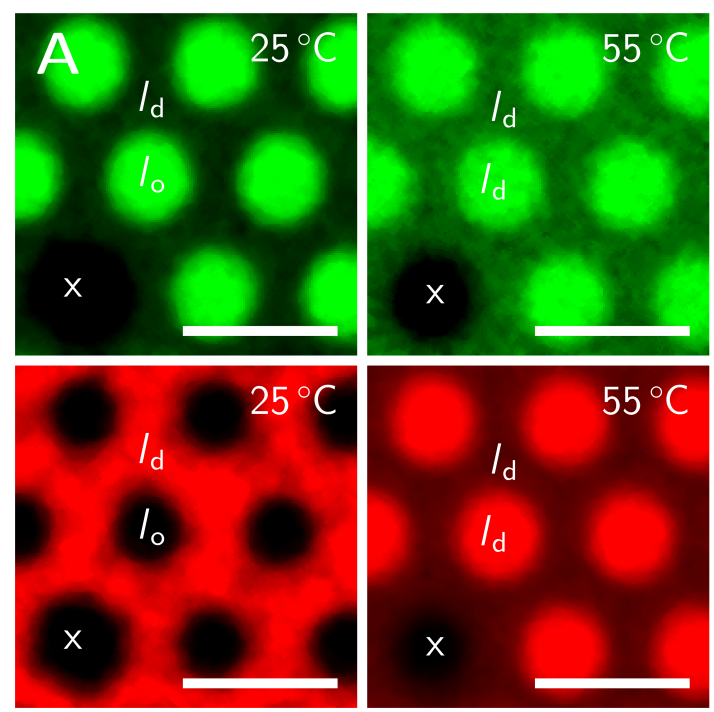
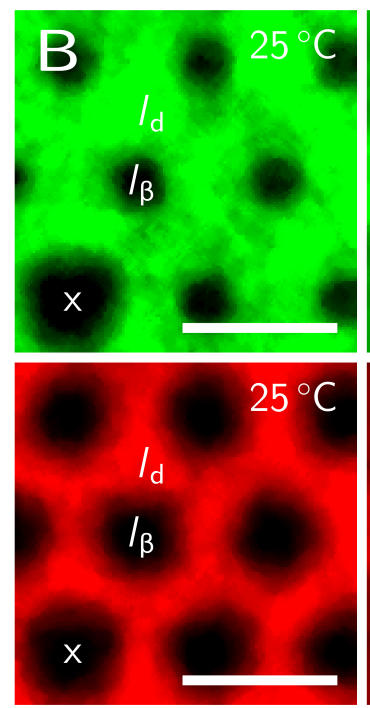

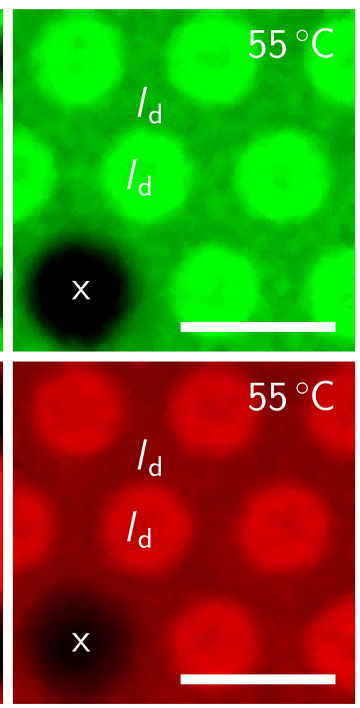

Figure 5.11.: Fluorescence micrographs of phase-separated pore-spanning membranes (PSMs) at $25^{\circ} \mathrm{C}$ and $55^{\circ} \mathrm{C}$. The green channel shows the BODIPY-Chol fluorescence, while the red channels shows the TexasRed-DHPE fluorescence. A) PSM composed of

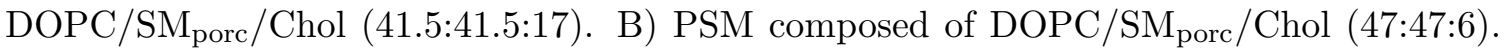
The gel like $\left(l_{\beta}\right)$, the liquid ordered $\left(l_{\mathrm{o}}\right)$ and the liquid disordered $\left(l_{\mathrm{d}}\right)$ phases are assigned to the freestanding pore-spanning membrane (f-PSM) and solid supported pore-spanning membrane (s-PSM) according to the partition of the two fluorophores BODIPY-Chol and TexasRed-DHPE. An uncovered pore is marked by an x. Scale bar $2 \mu \mathrm{m}$.

The f-PSMs showed a clear BODIPY-Chol fluorescence signal with higher Chol content (figure $5.11 \mathrm{~A}, T=25^{\circ} \mathrm{C}$ ), proving, that a f-PSM was still existent. The BODIPY-Chol fluorescence intensity of the s-PSM was still homogeneously distributed, but compared to the absolute intensity in the non-phase-separated state, the intensity level was lowered. The membrane composition DOPC/SM $\mathrm{Sorc}_{\text {c }} / \mathrm{Chol}$ (41.5:41.5: 17) excluded TexasRed-DHPE from the f-PSM while BODIPY-Chol was enriched, indicating that the f-PSM is composed of the $l_{\mathrm{o}}$ phase $\left.140 \mid 142\right]$ and the s-PSM of the $l_{\mathrm{d}}$ phase.

Interestingly, a mixture with low Chol content at $25^{\circ} \mathrm{C}$ showed no fluorescence intensity in the f-PSM at all, neither from the $l_{\mathrm{d}}$ nor from the $l_{\mathrm{o}}$ phase marker (figure $5.11 \mathrm{~B}, T=25^{\circ} \mathrm{C}$ ). By heating up the system to $55^{\circ} \mathrm{C}$, reaching the nonphase-separated state, both fluorophores were co-localized in the f-PSMs. This proves that even if no fluorescence is visible, a membrane suspending the pore is existent at $25^{\circ} \mathrm{C}$ and this membrane is excluding both fluorescence markers. Ternary phase 
diagrams of DOPC/SM/Chol do not only describe the $l_{\mathrm{d}}$ and $l_{\mathrm{o}}$ phase, but also the gel like $\left(l_{\beta}\right)$ phase which is present if the content of SM is very high. $97|29| 264|272| 275$ Both fluorescent lipid phase markers are excluded from the $l_{\beta}$ phase.140|170 At low Chol content the f-PSM was composed of the $l_{\beta}$ phase and the s-PSM was composed of the $l_{\mathrm{d}}$ phase.

Based on the assignment of the three detectable phases, temperature and Chol content were studied systematically to extract the transition temperature $\left(T_{\mathrm{M}}\right)$ by analyzing fluorescence images. The TexasRed-DHPE intensity was used to extract quantitative information, as TexasRed-DHPE is known to distribute to almost $100 \%$ in the $l_{\mathrm{d}}$ phase. $139 \mid 140$ In contrast, BODIPY-Chol partitions only about 50 to $80 \%$ into the $l_{\mathrm{o}}$ phase $\frac{142[229}{12}$ and was used primarily to distinguish between the $l_{\beta}$ and $l_{\mathrm{o}}$ phase. To extract the transition temperature, the ratio of fluorescence intensities of the f-PSM $\left(I_{\text {f-PSM }}\right)$ and s-PSM $\left(I_{\text {s-PSM }}\right)$ were measured for several temperatures, as explained in chapter 3.4.2. The intensity ratios were plotted against the temperature and showed a sigmoidal curve, where the turning point reflects the transition temperature.

The phase separation $l_{\beta} / l_{\mathrm{d}}$ is shown in figure 5.12 with the composition of DOPC/ $\mathrm{SM}_{\text {porc }} /$ Chol (46:46:8) along a rising temperature ramp. The fluorescence micrographs (figure 5.12, top, BODIPY-Chol (green) and TexasRed-DHPE (red)) at $T=25^{\circ} \mathrm{C}$ clearly showed that both fluorophores were excluded from the f-PSMs indicating that the f-PSMs were existent in the $l_{\beta}$ phase. TexasRed-DHPE fluorescence was found in the s-PSM representing the $l_{\mathrm{d}}$ phase. At $55^{\circ} \mathrm{C}$, a homogeneous distribution of the TexasRed-DHPE fluorophore in the f-PSM and in the s-PSM was observed. Hence, at Chol content of $8 \mathrm{~mol} \%$, a phase transition between a $l_{\beta} / l_{\mathrm{d}}$ phase-separated membrane to a homogeneous $l_{\mathrm{d}}$ phase occurred. From the plotted intensity ratio as a function of temperature (figure 5.12, bottom), a $T_{\mathrm{M}}$ of $40.7^{\circ} \mathrm{C}$ was extracted. This phase separation were observed for Chol contents of 0 to $10 \mathrm{~mol} \%$.

By increasing the Chol content to more than $10 \mathrm{~mol} \%$, the f-PSM formed a $l_{\mathrm{o}}$ phase instead of a $l_{\beta}$ phase. A PSM composed of DOPC/SM $\mathrm{SMrc}_{\text {pol }} / \mathrm{Chol}$ (42.5:42.5:15) is shown in figure 5.13. The fluorescence micrographs (figure 5.13, top, BODIPYChol (green) and TexasRed-DHPE (red)) at $T=25^{\circ} \mathrm{C}$ demonstrated that TexasRedDHPE was excluded from the f-PSM, while BODIPY-Chol was enriched proving the existence of the $l_{\mathrm{o}}$ phase in the f-PSM. TexasRed-DHPE fluorescence was found in the s-PSM, highlighting the $l_{\mathrm{d}}$ phase. At $55^{\circ} \mathrm{C}$, a homogeneous distribution of the TexasRed-DHPE in the f-PSM and in the s-PSM was observed. Hence, at a Chol content of $15 \mathrm{~mol} \%$, a phase transition between a $l_{\mathrm{o}} / l_{\mathrm{d}}$ phase-separated membrane to a homogeneous $l_{\mathrm{d}}$ phase occurred. From the plotted intensity ratio as a function 

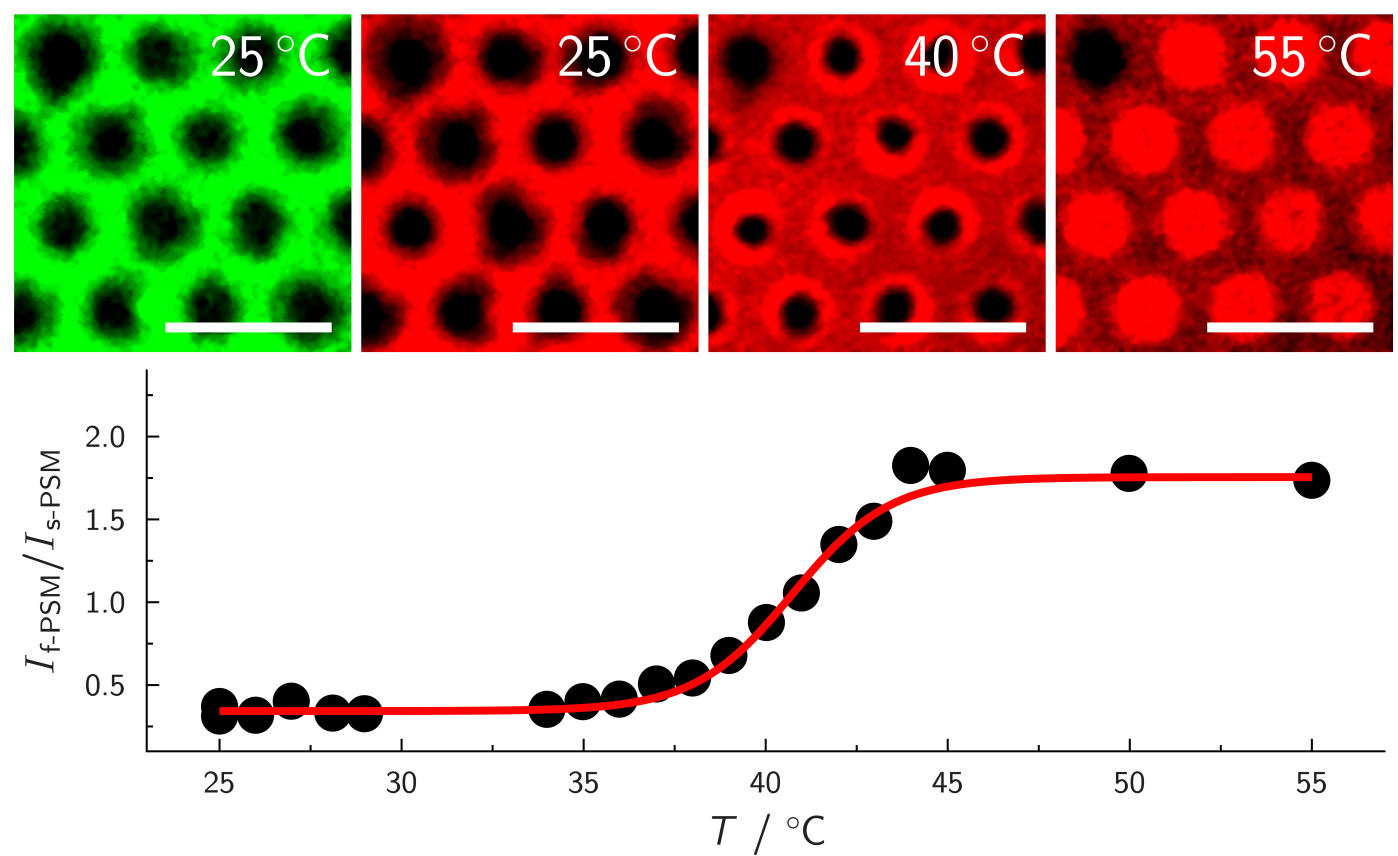

Figure 5.12.: (Top) Fluorescence micrographs of a PSM composed of DOPC/SM porc/ Chol (46:46:8) at different temperatures. The BODIPY-Chol (green) and the TexasRedDHPE (red) fluorescence images at $T=25^{\circ} \mathrm{C}$ indicate that the PSM phase-separates into a $l_{\beta}$ and $l_{\mathrm{d}}$ phase. The TexasRed-DHPE fluorescence images (red) at $T=25,40$ and $55^{\circ} \mathrm{C}$ demonstrate the phase transition from the $l_{\beta} / l_{\mathrm{d}}$ phase-separated membrane to a homogeneous $l_{\mathrm{d}}$ phase, with the visualization of phase separation in the f-PSM. (Bottom) Fluorescence intensity ratio as a function of temperature resulted in a sigmoidal curve which was fitted to the data resulting in $T_{\mathrm{M}}=40.7^{\circ} \mathrm{C}$. Scale bar $3 \mu \mathrm{m}$.

of temperature (figure 5.14, bottom), a $T_{\mathrm{M}}$ of $39.1^{\circ} \mathrm{C}$ was extracted. This phase separation behavior was observed for Chol contents of 10 to $50 \mathrm{~mol} \%$.

Another phase transition between a $l_{\mathrm{o}} / l_{\mathrm{d}}$ phase-separated membrane to a homogeneous $l_{\mathrm{d}}$ phase was shown in (figure 5.14) for a Chol content of $25 \mathrm{~mol} \%$. As shown in figure 5.13, the fluorescence micrographs (figure 5.14, top, BODIPY-Chol (green) and TexasRed-DHPE (red)) at $T=25^{\circ} \mathrm{C}$ demonstrate that TexasRed-DHPE was excluded from the f-PSM, while BODIPY-Chol was enriched assigning the f-PSM to the $l_{\mathrm{o}}$ phase and the s-PSM to the $l_{\mathrm{d}}$ phase. At $55^{\circ} \mathrm{C}$, a homogeneous distribution of the TexasRed-DHPE fluorophore in the f-PSM and in the s-PSM was observed. Varying the Chol contents between 0 and $18 \mathrm{~mol} \%$, a phase separation in the f-PSM was detected (figure 5.12 and 5.13). At higher Chol contents no phase separation in the f-PSM was found, during the temperature ramp measurement (figure 5.14 , top, TexasRed-DHPE (red) from 25 to $55^{\circ} \mathrm{C}$ ). The increase of the fluorescence intensity from TexasRed-DHPE in the f-PSM was measured and a $T_{\mathrm{M}}$ of $45.7^{\circ} \mathrm{C}$ was extracted. 

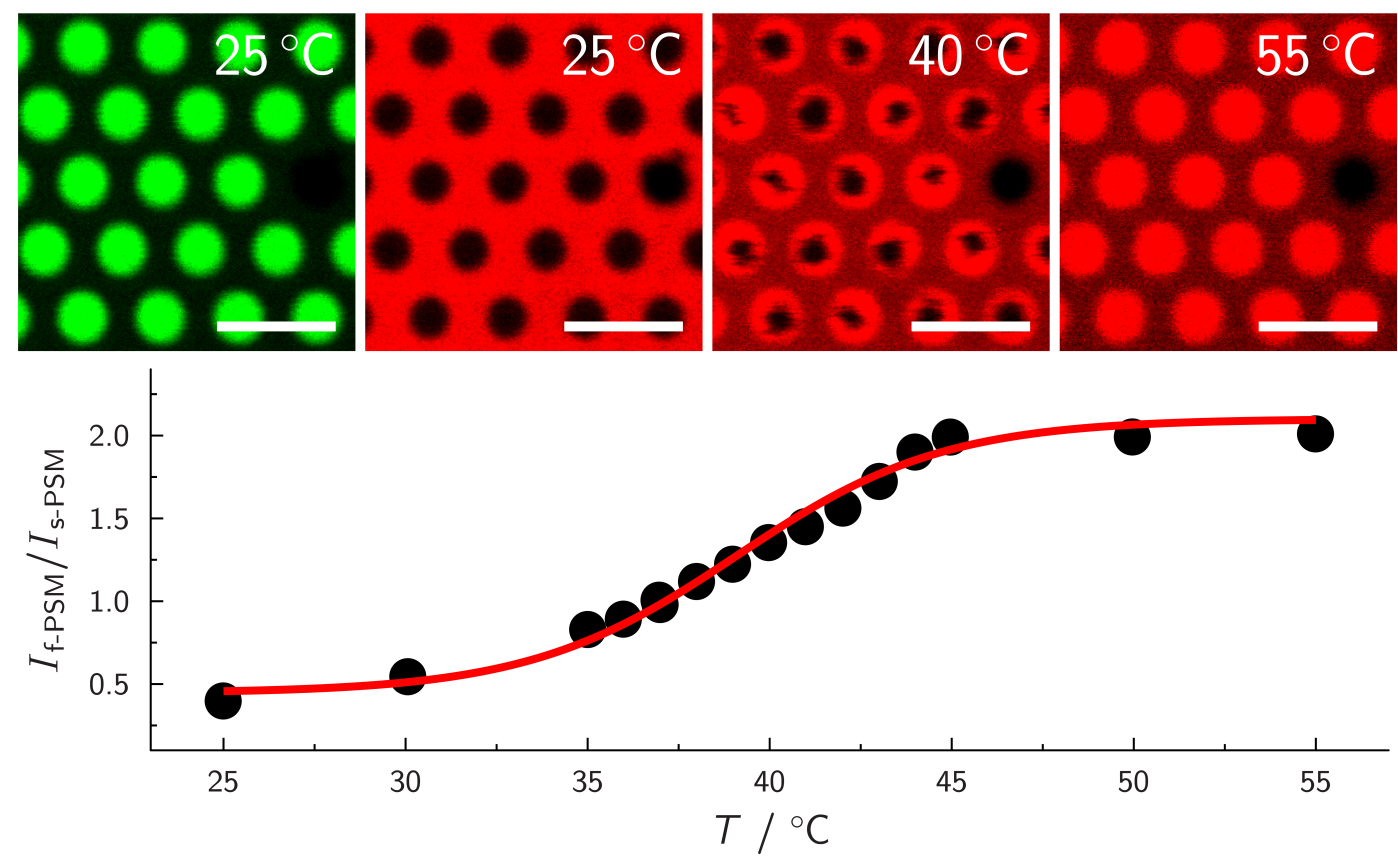

Figure 5.13.: (Top) Fluorescence micrographs of a PSM composed of DOPC/SM $/ \mathrm{Sorc}_{\text {por }}$ Chol (42.5:42.5:15) at different temperatures. The BODIPY-Chol (green) and the TexasRed-DHPE (red) fluorescence images at $T=25^{\circ} \mathrm{C}$ indicate that the PSM phase-separates into a $l_{\mathrm{o}}$ and $l_{\mathrm{d}}$ phase. The TexasRed-DHPE fluorescence images (red) at $T=25,40$ and $55^{\circ} \mathrm{C}$ demonstrate the phase transition from the $l_{\mathrm{o}} / l_{\mathrm{d}}$ phase-separated membrane to a homogeneous $l_{\mathrm{d}}$ phase, with the visualization of phase separation in the f-PSM. (Bottom) Fluorescence intensity ratio as a function of temperature resulted in a sigmoidal curve which was fitted to the data resulting in $T_{\mathrm{M}}=39.1^{\circ} \mathrm{C}$. Scale bar $3 \mu \mathrm{m}$.

$T_{\mathrm{MS}}$ for all measured Chol concentrations were plotted in the phase diagram (figure 5.15), indicating the border regions of the already described phase transitions which were possible in the PSM system. At high temperatures, the lipids were homogeneously distributed in the PSMs and independent from the Chol concentration. From $0 \mathrm{~mol} \% \mathrm{Chol}$ up to $8 \%$, only a $l_{\beta} / l_{\mathrm{d}}$ phase separation was found. From $11 \mathrm{~mol} \%$ to the highest measured Chol concentration, only a $l_{\mathrm{o}} / l_{\mathrm{d}}$ phase separation was detected. At concentrations of $9 \mathrm{~mol} \%$ and $10 \mathrm{~mol} \%$ Chol, both phase separation types were measured. This unclear border is a fact of the distribution of the GUVs which have up to $5 \%$ composition fluctuations within the population. $272|274| 276 \mid 277$ The highest used Chol concentration was $50 \mathrm{~mol} \%$ which is still below the maximum of soluble Chol that was determined to be $66 \mathrm{~mol} \%$ for DOPC membranes. 278|279] The upper limit of $50 \mathrm{~mol} \%$ for Chol was chosen to ensure that the Chol content in the membranes is unaffected by the maximum incorporation limit of Chol.

In previous literature, different phase diagrams from ternary lipid mixtures were reported on model membrane systems with a homogeneous membrane adhesion. 201 

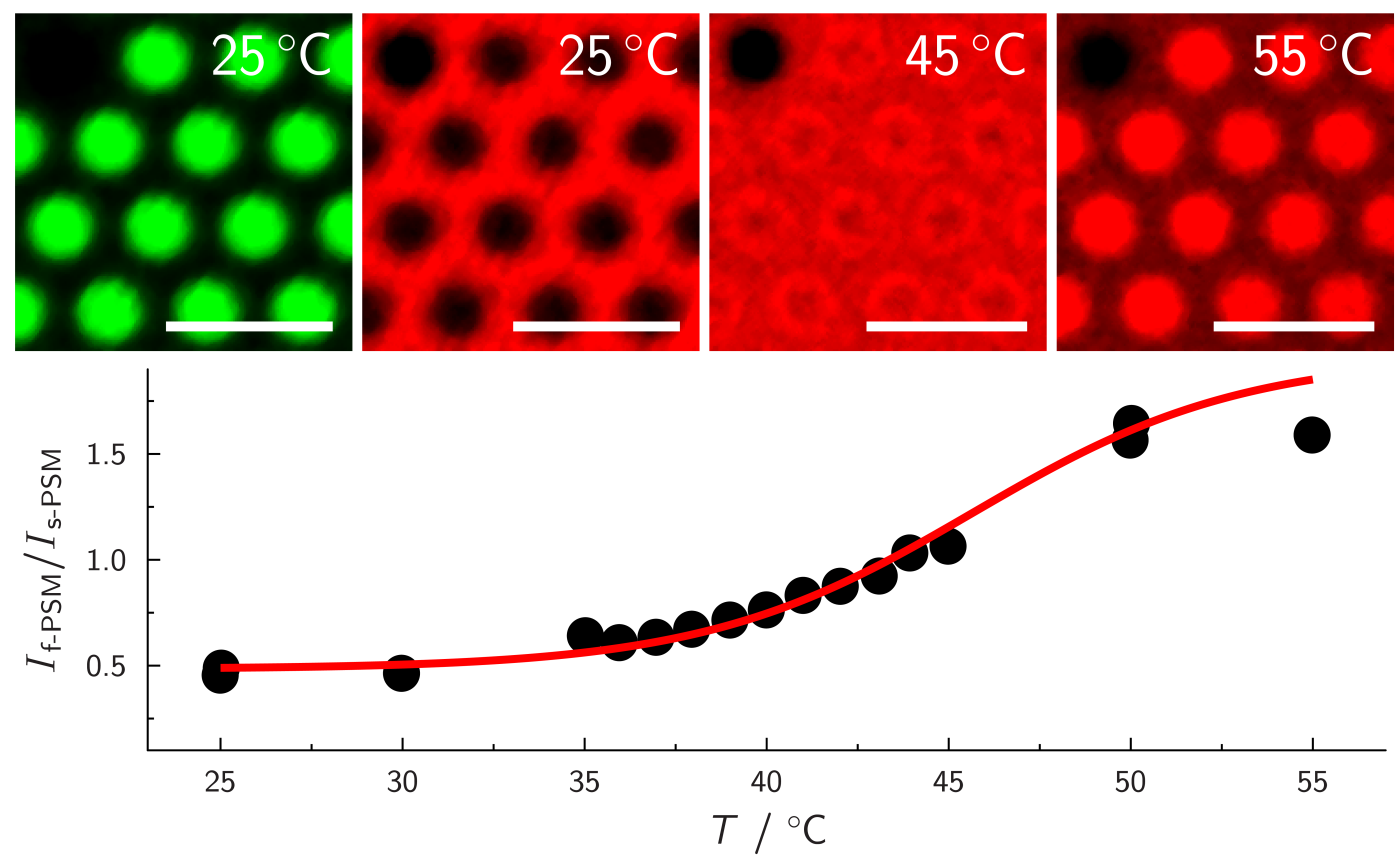

Figure 5.14.: (Top) Fluorescence micrographs of a PSM composed of DOPC/SM porc/ Chol (37.5:37.5:25) at different temperatures. The BODIPY-Chol (green) and the TexasRed-DHPE (red) fluorescence images at $T=25^{\circ} \mathrm{C}$ indicate that the PSM phase-separates into a $l_{\mathrm{o}}$ and $l_{\mathrm{d}}$ phase. The TexasRed-DHPE fluorescence images (red) at $T=25,40$ and $55{ }^{\circ} \mathrm{C}$ demonstrate the phase transition from the $l_{\mathrm{o}} / l_{\mathrm{d}}$ phase-separated membrane to a homogeneous $l_{\mathrm{d}}$ phase, without a visualization of phase separation in the f-PSM. (Bottom) Fluorescence intensity ratio as a function of temperature resulted in a sigmoidal curve which was fitted to the data resulting in $T_{\mathrm{M}}=45.7^{\circ} \mathrm{C}$. Scale bar $2 \mu \mathrm{m}$.

The difference of this measured phase diagram (figure 5.15) to ones reported for vesicles or SSM is explainable due to different adhesion properties for the membrane in PSM systems which basically have two different adhesion areas (f-PSM, s-PSM). A theoretical work from Lipowsky et al. described the influence of different adhesion areas on continuous membranes composed of POPC/Chol.11280. The phase diagram of the membrane in each adhesion region on its own is equal to the phase diagram of homogeneously adhered membranes. The overall phase diagram is changed, as different lipids show varying preferences to the different adhesion areas, resulting in a kind of lipid sorting induced by the adhesional properties of system. The deviations of the phase diagram for membrane systems with homogeneous adhesion to lipid membranes with heterogeneous adhesion can be summarized in three statements for the system used within this work: 1280 First, an increase in the difference of adhesional energy between f-PSM and s-PSM results in a broadening of the overall phase separation region within the phase diagram. Second, the maximum of $T_{\mathrm{M}}$ of the phase-separated region for the whole system does not change by increasing the difference of adhesional 


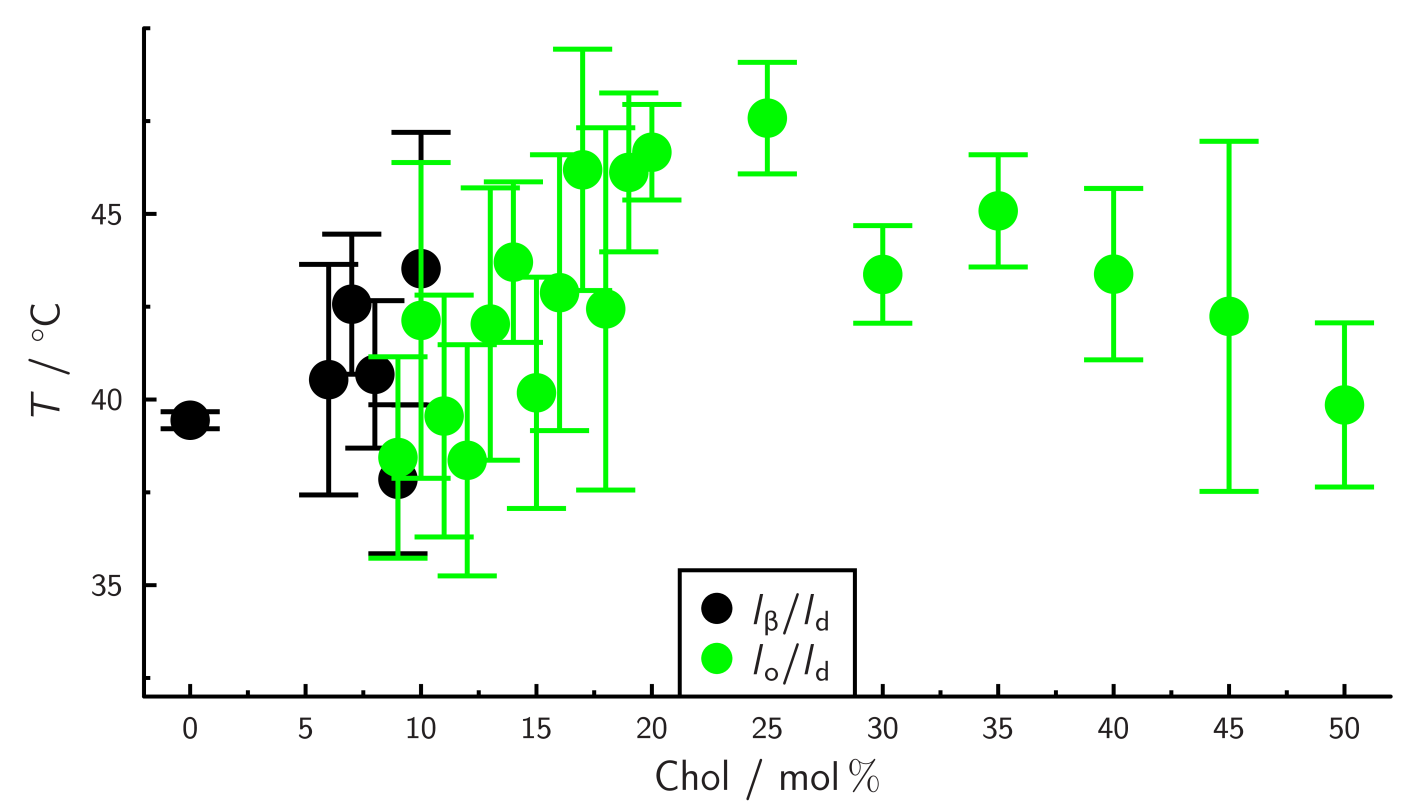

Figure 5.15.: hase diagram by changing the Chol content from 0 to $50 \mathrm{~mol} \%$ on porespanning membranes (PSMs) and the lipid ratio between DOPC and $\mathrm{SM}_{\text {porc }}$ was one. The different transition temperature $\left(T_{\mathrm{M}}\right)$ from gel like $\left(l_{\beta}\right)$ /liquid disordered $\left(l_{\mathrm{d}}\right)$ and liquid ordered $\left(l_{\mathrm{o}}\right) / l_{\mathrm{d}}$ to the homogeneous $l_{\mathrm{d}}$ phase were plotted in black and green respectively.

energy between f-PSM and s-PSM. Third, lipids have a preferential area (f-PSM, sPSM) depending on their properties within the lipid mixture which can result in differing lipid compositions for the f-PSM and s-PSM. Phase separation will occur either in the f-PSM or the s-PSM, but never in both simultaneously. 11280 These three theoretical statements are experimentally studied using the system of PSM as an example for heterogeneous adhesion and comparing these results to previous literature with experiments done with vesicles or SSMs as examples for homogeneously adhered membranes.

Starting with the first statement from Lipowsky et al., an increase in the adhesion difference must result in a broadening of the phase diagram 1280 which will be true if the preference of the different lipid phases to the different adhesion regions is stronger than the miscibility of the lipids, leading to the already described effect of lipid sorting. To prove this hypothesis an extreme point of phase separation is used for PSMs and compared to reported lipid mixtures with homogeneous membrane adhesion. Membranes with lipid compositions of DOPC/SM $\mathrm{Sorc}_{\text {p }} / \mathrm{Chol}$ with high Cholesterol content of $50 \mathrm{~mol} \%$ were used which showed a clear phase separation as seen in figure 5.15. The same lipid composition was demonstrated in a study of Petruzielo et al. using fluorescence resonance energy transfer (FRET) to investigate phase separation in multi-lamellar vesicles (MLVs).281 Phase separation 
was observed up to $40 \mathrm{~mol} \% \mathrm{Chol}, 281$ at higher Chol content no phase separation is reported. This indicates a broadening for this specific membrane mixture when different membrane areas with adhesional energy are present. Another phase diagram, measured with vesicles, was done by Carravilla et al..282 They analyzed the lipid composition by changing the $\mathrm{SM}$ from $\mathrm{SM}_{\text {porc }}$ to $\mathrm{SM}_{\text {egg }}$ and visualized the phase separation by Laudan in GUVs. They saw no phase separation with Chol contents higher than $40 \mathrm{~mol} \% .282$ Aufderhorst-Roberts et al. measured phase separation in the SSM using AFM to measure topographic images. In their work, it is reported that DOPC $/ \mathrm{SM}_{\text {egg }} /$ Chol membranes on mica showed phase separation up to $40 \mathrm{~mol} \%$ Chol at $25^{\circ} \mathrm{C} .283$ In all phase diagrams reported for homogeneous membrane adhesion, the phase-separated membrane was found up to $40 \mathrm{~mol} \%$ of Chol content but not for higher Chol contents. In the phase diagram of the whole PSMs, the phase separation was visible also at Chol contents of $50 \mathrm{~mol} \%$. This verifies the theoretical first hypothesis, namely that areas with differing adhesional energies lead to a broadening of the phase separation region in the phase diagram.

The second hypethesis from Lipowsky et al. is that a phase diagram of a lipid mixture with different membrane adhesion areas shows the same maximal transition temperature as a phase diagram of the same lipid mixture prepared with a homogeneous membrane adhesion. 1280 It is expected that the lipid mixture in the PSM can be sorted into two lipid compositions, one with high membrane adhesion in the s-PSM and one with no surface interaction in the f-PSM. The lipid composition in each of the both regions can be at different positions of the phase diagram, since the phase diagram describes the correct phase distribution behavior of f-PSM as well as s-PSM. If this is true, the maximum transition temperature should not change from each region individually and from the whole system compared to phase diagrams recorded for "classic" systems like SSMs or vesicles. Within this thesis, this statement cannot be verified in general, because only one line of a ternary phase diagram was measured. Still, it is possible to falsify this hypothesis. If in this thesis a phase transition temperature was measured, which is higher than a phase transition temperature in a phase diagram with homogeneous adhered membrane from a comparable lipid mixture, the statement would be rejected. The measurements of the transition temperatures of PSMs at the line DOPC/SM porc $/ \mathrm{Chol}(1: 1: \mathrm{x})$ had a maximum transition temperature of $47.5^{\circ} \mathrm{C}$. Many phase diagrams with a ternary lipid mixture were measured at one specific temperature. 201 The phase diagrams for DOPC/SM $\mathrm{C}_{16: 0} /$ Chol show a maximum $T_{\mathrm{M}}$ of around $50^{\circ} \mathrm{C} .99$ Pokorny et al. found phase separation for the lipid mixture POPC $/ \mathrm{SM}_{\text {porc }} / \mathrm{Chol}$ still at $46{ }^{\circ} \mathrm{C} .273$ Since in the literature for comparable lipid composition higher transition temperatures as on 
the PSMs are reported, the second statement from Lipowsky et al. is not rejected for this specific line in the ternary phase diagram but cannot be accepted in general. 1280

The last hypothesis by Lipowsky et al. describes competing process based on the local lipid composition concerning exchange of lipid material and phase separation. Since s-PSM and f-PSM are connected and able to exchange lipid material, the process of phase separation is not only dependent on temperature and overall membrane composition, but also on the local lipid composition which is prone to change upon lipid material exchange. The process of phase separation in either f-PSM or s-PSM is in competition with the process of exchanging local lipid compositions. Typically, the lipid composition would change before phase separation happens either in f-PSM or s-PSM. At a certain point, the energy cost of changing the lipid composition from the different adhesion regions is higher compared to one region, with the same membrane adhesion, undergoing a phase separation. This means that due to energetic reasons, phase separation is only possible in one of the regions and never in both at the same time. Phase separation was never observed in both regions (f-PSM and s-PSM) at the same time for the phase diagram measurements. Phase separation was only observed within the f-PSM in measurements with a Chol concentration of $\leq 18 \mathrm{~mol} \%$ (figure 5.12 and 5.13 at $40^{\circ} \mathrm{C}$ ). In contrast to this, the visualization of phase separation in both regions was possible if phase-separated GUVs were spread at room temperature (figure 5.4 and 5.5). This is not in contradiction to the hypothesis, since the membranes were kinetically entrapped and not in the thermodynamic equilibrium. This was proven, since heating the membranes above the transition temperature and cooling down lead to membranes without phase separation in both regions. The theory of Lipowsky et al. described only membranes in the thermodynamic equilibrium and not in a kinetic entrapment.1280 The theory of Lipowsky et al. is not falsified in general but verified for the lipid compositions used within this work.

s-PSM are composed of $l_{\mathrm{d}}$ phase and f-PSM of $l_{\mathrm{o}} / l_{\beta}$ phases at all different measured Chol concentrations. From the theory of Lipowsky et al.1280 and these findings, it is concluded that the adhesion energy to the $\mathrm{SiO}_{\mathrm{x}}$ functionalized substrates is higher for the $l_{\mathrm{d}}$ phase than for the $l_{\mathrm{o}}$ phase. This is in agreement with the measurements of the membrane tensions for the different phases. The results of membrane tension measurements on $\mathrm{SiO}_{\mathrm{x}}$ functionalized substrates suggest that the adhesion energy for the $l_{\mathrm{d}}$ phase is higher compared to the adhesion energy for the $l_{\mathrm{o}}$ phase (chapter 5.1.2. page 75). The reason is that the membrane bending stiffness of the $l_{\mathrm{d}}$ phase is lower compared to the $l_{\mathrm{o}}$ phase. 243254 Therefore, more surface on the rough $\mathrm{SiO}_{\mathrm{x}}$ functionalized substrate, for the membrane adhesion is present for the $l_{\mathrm{d}}$ phase. 


\subsection{Conclusion of pore-spanning membranes (PSMs)}

As found in cellular systems, where the membrane is partly adhered and partly free standing, the PSM models allow to systematically study the influence of adhesion in complex membrane systems. The interaction of membrane and substrate in the s-PSM region influences the membrane properties in the f-PSM region. With higher adhesion in the s-PSM region, the diffusion coefficient in the s-PSM slowed down and the diffusion coefficient in the f-PSM increased as determined by iSCAT measurements. The adhesion of the membrane to the substrate in the s-PSM is the origin of the membrane tension in the f-PSM. The functionalization with gold and different mercaptoalcohols resulted in the tension regime of 0.6 to $3.2 \mathrm{mN} \mathrm{m}^{-1}$. While p- $\mathrm{Si}_{3} \mathrm{~N}_{4}$ functionalized surfaces showed a tension of $1.6 \pm 0.5 \mathrm{mN} \mathrm{m}^{-1}$, a tension of $10.5 \pm 4.3 \mathrm{mN} \mathrm{m}^{-1}$ was found when $\mathrm{SiO}_{\mathrm{x}}$ functionalized substrates were used. The $\mathrm{SiO}_{\mathrm{x}}$ surfaces allow the detection of fluorescence signals in f-PSMs as well as s-PSMs and were used to study phase-separated PSMs in dependency of the two regions. The $l_{\mathrm{d}}$ and $l_{\mathrm{o}}$ phases can be distinguished with a factor of 20 by FCS measurements in the f-PSM.

$\mathrm{STxB} \mathrm{Gb}_{3}$ binding was not effected by the high membrane tension or the high substrate adhesion of the membrane to the $\mathrm{SiO}_{\mathrm{x}}$ functionalized substrate, because it binds to the natural $l_{\mathrm{o}}$ phase (figure 5.5).

To investigate the influence of the different adhesion properties on the phaseseparated membranes, a phase diagram was measured by increasing the Chol content in a membrane with an equimolar of DOPC and $\mathrm{SM}_{\text {porc }}$. Two different fluorescent phase markers were used to determine the lipid phases in the f-PSMs and s-PSMs for different temperatures and Chol contents. As it was shown for a low Chol content $(\leq 10 \mathrm{~mol} \%)$, the phase separation takes place between the $l_{\beta}$ phase in the f-PSM and the $l_{\mathrm{d}}$ phase in the s-PSM at low temperatures $\left(<30^{\circ} \mathrm{C}\right)$ to a homogeneous $l_{\mathrm{d}}$ phase in the whole PSM at high temperatures $\left(>50^{\circ} \mathrm{C}\right)$. Another phase separation takes place at a high Chol content $(\geq 9 \mathrm{~mol} \%)$ from a phase-separated PSM with the $l_{\mathrm{o}}$ phase in the f-PSM and the $l_{\mathrm{d}}$ phase in the s-PSM to a homogeneous $l_{\mathrm{d}}$ phase in the whole PSM. Phase diagrams with a homogeneous adhesion to the membrane showed no phase-separated membrane with $50 \mathrm{~mol} \%$ Chol. In contrast to this finding, the phase diagram of the PSM showed phase-separated membranes at that Chol content. This confirms the broadening effect of the phase-separated region in the phase diagram from the theoretical work of Lipowsky et al..1280

The cell membrane can be described with the lipid raft model and this model includes a clustering of the membrane. The results of the phase-separated PSMs 
gave a hint that not only the lipid-protein mixture has an effect on the membrane ordering, but also that the different pining points affect the membrane ordering, membrane dynamic and lipid-protein distribution. 


\section{Conclusion}

Enterohemorrhagic Escherichia coli (EHEC) is a bacterial strain, causing diseases like diarrhea, bloody diarrhea, hemorrhagic colitis, haemolytic uraemic syndrome and damage of the central nervous system. All these diseases are triggered by shiga toxin (STx) which is a protein produced by EHEC. STx is known to bind to globotriaosyl ceramide $\left(\mathrm{Gb}_{3}\right)$ in the cellular membranes. After binding, STx enters the cell where it inhibits the intracellular protein biosynthesis. The B-subunit of STx (STxB) is responsible for binding while the A-subunit of STx (STxA) inflicts the toxic effect. The STxB is known to bind to the more ordered membrane structure at the cells, but the distribution of $\mathrm{Gb}_{3}$ before the $\mathrm{STxB}$ binding is not known which was investigated in this work. Biomimetic model membranes showing liquid ordered $\left(l_{\mathrm{o}}\right)$ /liquid disordered $\left(l_{\mathrm{d}}\right)$ phase separation were employed to study $\mathrm{Gb}_{3}$ phase performance before and after STxB binding.

To quantify the partitioning of $\mathrm{Gb}_{3}$ in coexisting $l_{\mathrm{o}} / l_{\mathrm{d}}$ phases, fluorescently fatty acid labeled $\mathrm{Gb}_{3} \mathrm{~s}$ were synthesized, containing pentaene or hexaene moieties that allowed localization of $\mathrm{Gb}_{3}$ molecules in $l_{\mathrm{o}} / l_{\mathrm{d}}$ membranes by measure of fluorescence microscopy. These $\mathrm{Gb}_{3}$ s were preferentially localized in the $l_{\mathrm{d}}$ phase. STxB binding to these $\mathrm{Gb}_{3}$-species also takes place in the $l_{\mathrm{d}}$ phase which contradicts the binding behavior of the natural $\mathrm{Gb}_{3}$. To contain a natural phase preference of $\mathrm{Gb}_{3}$, a second labeling strategy was employed by connecting a BODIPY fluorophore to the head group of the $\mathrm{Gb}_{3}$ using polyethylene glycol (PEG) linker. The synthesized head group labeled $\mathrm{Gb}_{3} \mathrm{~s}$ allowed to address the question how the structure of the fatty acid of $\mathrm{a} \mathrm{Gb}_{3}$ influences its distribution in $l_{\mathrm{o}} / l_{\mathrm{d}}$ phase-separated membranes. The results clearly demonstrate that the unsaturated fatty acid significantly shifts the $\mathrm{Gb}_{3}$ molecules from the $l_{\mathrm{o}}$ phase to the $l_{\mathrm{d}}$ phase. Which can be explained because the $l_{\mathrm{o}}$ phase is enriched in saturated lipids and the $l_{\mathrm{d}}$ phase enriched unsaturated lipids. An $\alpha$-hydroxilation at the fatty acid showed no effect an the distribution of $\mathrm{Gb}_{3}$. Furthermore, the fatty acids of sphingomyelin (SM) impact the distribution of the $\mathrm{Gb}_{3}$ due to interdigitation. It is conceivable that the overall recruitment of lipids and the STx induced membrane reorganization eventually leads to invagination 
of the protein into the host cells and that this can be an influence of the fatty acid composition of $\mathrm{Gb}_{3}$.

The cell membrane has different interactions like cell-cell junctions, focal adhesions or the cytoskeleton. These adhesional points might influence local lipid compartition and therefore could have an impact for the lipid raft hypothesis and also on the $\mathrm{Gb}_{3}$ distribution in the cellular membrane. A model membrane system with different adhesional energies to the membrane is the pore-spanning membrane (PSM). While established PSM system are based on gold thiol functionalization. The gold prohibit fluorescence readout in the solid supported PSM (s-PSM). Within this thesis, a silicon oxide $\left(\mathrm{SiO}_{\mathrm{x}}\right)$ functionalization was established and characterized to enable phase separation on freestanding PSM (f-PSM) and s-PSM.

To investigate the influence of different adhesional properties on phase-separated membranes, a phase diagram was obtained by increasing the cholesterol (Chol) content in an equimolar membrane composed of DOPC and $\mathrm{SM}_{\text {porc }}$. The existence of two different adhesion areas (s-PSM and f-PSM) had a dramatic effect on the phase diagram. The region of the phase-separated membrane was broadened in comparison to phase diagrams reported from systems with homogeneous membrane adhesion. Interestingly, the $l_{\mathrm{d}}$ phase was only observed in the s-PSM, while the more ordered membrane was found in the f-PSM. The adhesion of the $l_{\mathrm{d}}$ phase to the rough surface can be favored, as the lower bending energy allows for the $l_{\mathrm{d}}$ phase to get higher adhesion energy.

The results obtain in this thesis suggest that not only the lipid-protein mixture has an effect on the membrane reorganization to nano domains, but also the adhesional points affected the membrane reorganization, membrane dynamic and lipid-protein distribution. This can also influence the phase distribution of the $\mathrm{Gb}_{3}$ which can be analyzed in further steps using PSMs. This would provide a better biomimetic model compared to giant unilamellar vesicles (GUVs). 


\section{References}

1. Lipowsky R., Rouhiparkouhi T., Discher D. E. \& Weikl T. R. Domain formation in cholesterol-phospholipid membranes exposed to adhesive surfaces or environments. Soft Matter 9, 8438-8453 (2013).

2. Scherer W. F., Syverton J. T. \& Gey G. O. Studies on the propagation in vitro of poliomyelitis viruses: IV. Viral multiplication in a stable strain of human malignant epithelial cells (strain HeLa) derived from an epidermoid carcinoma of the cervix. J. Exp. Med. 97, 695-710 (1953).

3. Michino H., Araki K., Minami S., Takaya S., Sakai N., Miyazaki M., Ono A. \& Yanagawa H. Massive outbreak of Escherichia coli O157: H7 infection in schoolchildren in Sakai City, Japan, associated with consumption of white radish sprouts. Am. J. Epidemiol. 150, 787-796 (1999).

4. Baljer G. \& Wieler L. Enterohaemorrhagic E. coli (EHEC)-recent information on a new medically important zoonotic angent. Lohman Information 22, 21-6 (1999).

5. Bettelheim K. A., Whipp M., Djordjevic S. P. \& Ramachandran V. First isolation outside Europe of sorbitol-fermenting verocytotoxigenic Escherichia coli (VTEC) belonging to O group O157. J. Med. Microbiol. 51, 713 (2002).

6. Croxen M. A., Law R. J., Scholz R., Keeney K. M., Wlodarska M. \& Finlay B. B. Recent advances in understanding enteric pathogenic Escherichia coli. Clin. Microbiol. Rev. 26, 822-880 (2013).

7. Konowalchuk J., Speirs J. \& Stavric S. Vero response to a cytotoxin of Escherichia coli. Infect. Immun. 18, 775-779 (1977).

8. Riley L. W., Remis R. S., Helgerson S. D., McGee H. B., Wells J. G., Davis B. R., Hebert R. J., Olcott E. S., Johnson L. M., Hargrett N. T., et al. Hemorrhagic colitis associated with a rare Escherichia coli serotype. New Engl. J. Med. 308, 681-685 (1983). 
9. Grant J., Wendelboe A. M., Wendel A., Jepson B., Torres P., Smelser C. \& Rolfs R. T. Spinach-associated Escherichia coli O157: H7 outbreak, Utah and New Mexico, 2006. Emerg. Infect. Dis. 14, 1633 (2008).

10. Sartz L., De Jong B., Hjertqvist M., Plym-Forshell L., Alsterlund R., Löfdahl S., Osterman B., Ståhl A., Eriksson E., Hansson H.-B., et al. An outbreak of Escherichia coli O157: H7 infection in southern Sweden associated with consumption of fermented sausage; aspects of sausage production that increase the risk of contamination. Epidemiol. Infect. 136, 370-380 (2008).

11. Schimmer B., Nygard K., Eriksen H.-M., Lassen J., Lindstedt B., Brandal L. T., Kapperud G. \& Aavitsland P. Outbreak of haemolytic uraemic syndrome in Norway caused by stx 2-positive Escherichia coli O103: H25 traced to cured mutton sausages. BMC Infect. Dis. 8, 41 (2008).

12. Ammon A., Petersen L. R. \& Karch H. A Large Outbreak of Hemolytic Uremic Syndrome Caused by an Unusual Sorbitol-Fermenting Strain of Escherichia coli O157: H-. J. Infect. Dis. 179, 1274-1277 (1999).

13. Buchholz U., Bernard H., Werber D., Böhmer M. M., Remschmidt C., Wilking H., Deleré Y., an der Heiden M., Adlhoch C., Dreesman J., et al. German outbreak of Escherichia coli O104: H4 associated with sprouts. New Engl. J. Med. 365, 1763-1770 (2011).

14. Bielaszewska M., Mellmann A., Zhang W., Köck R., Fruth A., Bauwens A., Peters G. \& Karch H. Characterisation of the Escherichia coli strain associated with an outbreak of haemolytic uraemic syndrome in Germany, 2011: a microbiological study. Lancet Infect. Dis. 11, 671-676 (2011).

15. Karch H. EHEC O104: H4 und die Folgen. Biospektrum 17, 616-620 (2011).

16. Appel B., Böl G., Greiner M., Lahrssen-Wiederholt M. \& Hensel A. EHEC outbreak 2011. Investigation of the outbreak along the food chain. Berlin: Bundesinstitut für Risikobewertung (2012).

17. Burger R. in Improving Food Safety Through a One Health Approach: Workshop Summary, 2012 115-130 (Robert Koch-Institut, 2012).

18. Frank C., Faber M., Askar M., Bernard H., Fruth A., Gilsdorf A., Höhle M., Karch H., Krause G., Prager R., et al. Large and ongoing outbreak of haemolytic uraemic syndrome, Germany, May 2011. Eurosurveillance 16, 19878 (2011).

19. Alpers K., Stark K., Hellenbrand W. \& Ammon A. Zoonotische Infektionen beim Menschen. Bundesgesundheitsbla. 47, 622-632 (2004). 
20. Robert Koch-Institut. SurvStat@RKI 2.0 German. https://survstat.rki. de (July 30, 2019).

21. Villysson A., Tontanahal A. \& Karpman D. Microvesicle Involvement in Shiga Toxin-Associated Infection. Toxins 9, 376 (2017).

22. Onyenweaku Florence C., Ifeanyi O. E. \& Nwandikor Uzoije U. A Review on Heamolytic Uremic Syndrome. Int. J. Curr. Res. Med. Sci. 4, 80-92 (2018).

23. Tarr P. I., Gordon C. A. \& Chandler W. L. Shiga-toxin-producing Escherichia coli and haemolytic uraemic syndrome. Lancet 365, 1073-1086 (2005).

24. Spinale J. M., Ruebner R. L., Copelovitch L. \& Kaplan B. S. Long-term outcomes of Shiga toxin hemolytic uremic syndrome. Pediatr. Nephrol. 28, 20972105 (2013).

25. Melton-Celsa A. R. Shiga toxin (Stx) classification, structure, and function. Microbiol. Spectrum 2 (2014).

26. Tesh V. L., Burris J., Owens J., Gordon V., Wadolkowski E., O’brien A. \& Samuel J. Comparison of the relative toxicities of Shiga-like toxins type I and type II for mice. Infect. Immun. 61, 3392-3402 (1993).

27. Wadolkowski E., Sung L., Burris J., Samuel J. \& O’brien A. Acute renal tubular necrosis and death of mice orally infected with Escherichia coli strains that produce Shiga-like toxin type II. Infect. Immun. 58, 3959-3965 (1990).

28. O’Brien A., Lively T., Chen M., Rothman S. \& Formal S. Escherichia coli 0157: H7 strains associated with haemorrhagic colitis in the United States produce a Shigella dysenteriae 1 (Shiga) like cytotoxin. Lancet 321, 702 (1983).

29. Manitz J., Kneib T., Schlather M., Helbing D. \& Brockmann D. Origin detection during food-borne disease outbreaks-a case study of the 2011 ehec/hus outbreak in germany. PLoS Curr. 6 (2014).

30. Trachtman H., Austin C., Lewinski M. \& Stahl R. A. Renal and neurological involvement in typical Shiga toxin-associated HUS. Nat. Rev. Nephrol. 8, 658 (2012).

31. Karch H., Tarr P. I. \& Bielaszewska M. Enterohaemorrhagic Escherichia coli in human medicine. Int. J. Med. Microbiol. 295, 405-418 (2005).

32. Karpman D., Loos S., Tati R. \& Arvidsson I. Haemolytic uraemic syndrome. J. Intern. Med. 281, 123-148 (2017). 
33. Exeni R. A., Fernandez-Brando R. J., Santiago A. P., Fiorentino G. A., Exeni A. M., Ramos M. V. \& Palermo M. S. Pathogenic role of inflammatory response during Shiga toxin-associated hemolytic uremic syndrome (HUS). Pediatr. Nephrol. 1-15 (2018).

34. Obata F., Tohyama K., Bonev A. D., Kolling G. L., Keepers T. R., Gross L. K., Nelson M. T., Sato S. \& Obrig T. G. Shiga toxin 2 affects the central nervous system through receptor globotriaosylceramide localized to neurons. J. Infect. Dis. 198, 1398-1406 (2008).

35. Friedrich A. W., Bielaszewska M., Zhang W.-L., Pulz M., Kuczius T., Ammon A. \& Karch H. Escherichia coli harboring Shiga toxin 2 gene variants: frequency and association with clinical symptoms. J. Infect. Dis. 185, 74-84 (2002).

36. Fuller C. A., Pellino C. A., Flagler M. J., Strasser J. E. \& Weiss A. A. Shiga toxin subtypes display dramatic differences in potency. Infect. Immun. 79, 1329-1337 (2011).

37. Shiga K. Studien über die epidemische Dysenterie in Japan, unter besonderer Berücksichtigung des Bacillus dysenteriae (Schluss aus No. 44.) Deut. Med. Wochenschr. 27, 783-786 (1901).

38. Orihuel A., Terán L., Renaut J., Planchon S., Valacco M. P., Masias E., Minahk C., Vignolo G., Moreno S., De Almeida A. M., et al. Physiological and proteomic response of Escherichia coli O157: H7 to a bioprotective lactic acid bacterium in a meat environment. Food Res. Int. 108622 (2019).

39. Zoja C., Buelli S. \& Morigi M. Shiga toxin-associated hemolytic uremic syndrome: pathophysiology of endothelial dysfunction. Pediatr. Nephrol. 25, 2231$2240(2010)$.

40. Lee M.-S. \& Tesh V. L. Roles of Shiga Toxins in Immunopathology. Toxins 11, $212(2019)$.

41. Mahfoud R., Manis A. \& Lingwood C. A. Fatty acid-dependent globotriaosyl ceramide receptor function in detergent resistant model membranes. J. Lipid Res. 50, 1744-1755 (2009).

42. Mahfoud R., Manis A., Binnington B., Ackerley C. \& Lingwood C. A. A major fraction of glycosphingolipids in model and cellular cholesterol-containing membranes is undetectable by their binding proteins. J. Biol. Chem. 285, 36049-36059 (2010). 
43. Lindberg A., Brown J., Strömberg N., Westling-Ryd M., Schultz J. \& Karlsson K. Identification of the carbohydrate receptor for Shiga toxin produced by Shigella dysenteriae type 1. J. Biol. Chem. 262, 1779-1785 (1987).

44. Ramegowda B., Samuel J. E. \& Tesh V. L. Interaction of Shiga toxins with human brain microvascular endothelial cells: cytokines as sensitizing agents. $J$. Infect. Dis. 180, 1205-1213 (1999).

45. Legros N., Pohlentz G., Runde J., Dusny S., Humpf H.-U., Karch H. \& Müthing J. Colocalization of receptors for Shiga toxins with lipid rafts in primary human renal glomerular endothelial cells and influence of D-PDMP on synthesis and distribution of glycosphingolipid receptors. Glycobiology 27, 947-965 (2017).

46. Legros N., Dusny S., Humpf H.-U., Pohlentz G., Karch H. \& Müthing J. Shiga toxin glycosphingolipid receptors and their lipid membrane ensemble in primary human blood-brain barrier endothelial cells. Glycobiology 27, 99-109 (2016).

47. Legros N., Pohlentz G., Steil D. \& Müthing J. Shiga toxin-glycosphingolipid interaction: Status quo of research with focus on primary human brain and kidney endothelial cells. Int. J. Med. Microbiol. 308, 1073-1084 (2018).

48. Steil D., Bonse R., Meisen I., Pohlentz G., Vallejo G., Karch H. \& Müthing J. A topographical atlas of Shiga toxin 2e receptor distribution in the tissues of weaned piglets. Toxins 8, 357 (2016).

49. Kale R. R., McGannon C. M., Fuller-Schaefer C., Hatch D. M., Flagler M. J., Gamage S. D., Weiss A. A. \& Iyer S. S. Differentiation between structurally homologous Shiga 1 and Shiga 2 toxins by using synthetic glycoconjugates. Angew. Chem. Int. Ed. 47, 1265-1268 (2008).

50. Ling H., Boodhoo A., Hazes B., Cummings M. D., Armstrong G. D., Brunton J. L. \& Read R. J. Structure of the shiga-like toxin I B-pentamer complexed with an analogue of its receptor $\mathrm{Gb}_{3}$. Biochemistry 37, 1777-1788 (1998).

51. Jacobson J. M., Yin J., Kitov P. I., Mulvey G., Griener T. P., James M. N., Armstrong G. \& Bundle D. R. The crystal structure of Shiga toxin type 2 with bound disaccharide guides the design of a heterobifunctional toxin inhibitor. J. Biol. Chem. 289, 885-894 (2014).

52. Mobassaleh M., Donohue-Rolfe A., Jacewicz M., Grand R. J. \& Keusch G. T. Pathogenesis of shigella diarrhea: evidence for a developmentally regulated glycolipid receptor for shigella toxin involved in the fluid secretory response of rabbit small intestine. J. Infect. Dis. 157, 1023-1031 (1988). 
53. Reisbig R., Olsnes S. \& Eiklid K. The cytotoxic activity of Shigella toxin. Evidence for catalytic inactivation of the $60 \mathrm{~S}$ ribosomal subunit. J. Biol. Chem. 256, 8739-8744 (1981).

54. Windschiegl B., Orth A., Romer W., Berland L., Stechmann B., Bassereau P., Johannes L. \& Steinem C. Lipid reorganization induced by Shiga toxin clustering on planar membranes. PLoS One 4, e6238 (2009).

55. Safouane M., Berland L., Callan-Jones A., Sorre B., Römer W., Johannes L., Toombes G. E. \& Bassereau P. Lipid cosorting mediated by shiga toxin induced tubulation. Traffic 11, 1519-1529 (2010).

56. Pezeshkian W., Hansen A. G., Johannes L., Khandelia H., Shillcock J., Kumar P. S. \& Ipsen J. Membrane invagination induced by Shiga toxin B-subunit: from molecular structure to tube formation. Soft Matter 12, 5164-5171 (2016).

57. Römer W., Berland L., Chambon V., Gaus K., Windschiegl B., Tenza D., Aly M. R., Fraisier V., Florent J.-C., Perrais D., Lamaze C., Raposo G., Steinem C., Sens P., Bassereau P. \& Johannes L. Shiga toxin induces tubular membrane invaginations for its uptake into cells. Nature 450, 670-675 (2007).

58. Sandvig K., Olsnes S., Brown J. E., Petersen O. W. \& van Deurs B. Endocytosis from coated pits of Shiga toxin: a glycolipid-binding protein from Shigella dysenteriae 1. J. Cell Biol. 108, 1331-1343 (1989).

59. Sandvig K. \& Van Deurs B. Endocytosis, intracellular transport, and cytotoxic action of Shiga toxin and ricin. Physiol. Rev. 76, 949-966 (1996).

60. Lauvrak S. U., Torgersen M. L. \& Sandvig K. Efficient endosome-to-Golgi transport of Shiga toxin is dependent on dynamin and clathrin. J. Cell Sci. 117, 2321-2331 (2004).

61. Saint-Pol A., Yélamos B., Amessou M., Mills I. G., Dugast M., Tenza D., Schu P., Antony C., McMahon H. T., Lamaze C., et al. Clathrin adaptor epsinR is required for retrograde sorting on early endosomal membranes. Dev. Cell 6, 525-538 (2004).

62. Johannes L. \& Römer W. Shiga toxins from cell biology to biomedical applications. Nat. Rev. Microbiol. 8, 105-116 (2010).

63. Spooner R. A. \& Lord J. M. in Ricin and Shiga Toxins 19-40 (Springer, 2011).

64. Johannes L. Shiga Toxin-A Model for Glycolipid-Dependent and Lectin-Driven Endocytosis. Toxins 9, 340 (2017). 
65. Kovbasnjuk O., Mourtazina R., Baibakov B., Wang T., Elowsky C., Choti M. A., Kane A. \& Donowitz M. The glycosphingolipid globotriaosylceramide in the metastatic transformation of colon cancer. Proc. Natl. Acad. Sci. U.S.A. 102, 19087-19092 (2005).

66. Amessou M., Carrez D., Patin D., Sarr M., Grierson D. S., Croisy A., Tedesco A. C., Maillard P. \& Johannes L. Retrograde delivery of photosensitizer (TPPpO- $\beta$-GluOH) 3 selectively potentiates its photodynamic activity. Bioconjugate Chem. 19, 532-538 (2008).

67. Distler U., Souady J., Hülsewig M., Drmić-Hofman I., Haier J., Friedrich A. W., Karch H., Senninger N., Dreisewerd K., Berkenkamp S., et al. Shiga toxin receptor $\mathrm{Gb}_{3} \mathrm{Cer} / \mathrm{CD} 77$ : tumor-association and promising therapeutic target in pancreas and colon cancer. PLoS One 4, e6813 (2009).

68. Engedal N., Skotland T., Torgersen M. L. \& Sandvig K. Shiga toxin and its use in targeted cancer therapy and imaging. Microb. Biotechnol. 4, 32-46 (2011).

69. Luginbuehl V., Meier N., Kovar K. \& Rohrer J. Intracellular drug delivery: Potential usefulness of engineered Shiga toxin subunit B for targeted cancer therapy. Biotechnol. Adv. 36, 613-623 (2018).

70. LaCasse E., Bray M., Patterson B., Lim W.-M., Perampalam S., Radvanyi L., Keating A., Stewart A., Buckstein R., Sandhu J., Miller N., Banerjee D., Singh D., Belch A., Pilarski L. \& Gariépy J. Shiga-like toxin-1 receptor on human breast cancer, lymphoma, and myeloma and absence from CD34+ hematopoietic stem cells: implications for ex vivo tumor purging and autologous stem cell transplantation. Blood 94, 2901-2910 (1999).

71. Tarragó-Trani M. T. \& Storrie B. Alternate routes for drug delivery to the cell interior: pathways to the Golgi apparatus and endoplasmic reticulum. Adv. Drug Deliver. Rev. 59, 782-797 (2007).

72. Batisse C., Dransart E., Sarkouh R. A., Brulle L., Bai S.-K., Godefroy S., Johannes L. \& Schmidt F. A new delivery system for auristatin in STxB-drug conjugate therapy. Eur. J. Med. Chem. 95, 483-491 (2015).

73. Maak M., Nitsche U., Keller L., Wolf P., Sarr M., Thiebaud M., Rosenberg R., Langer R., Kleeff J., Friess H., et al. Tumor-specific targeting of pancreatic cancer with Shiga toxin B-subunit. Mol. Cancer Ther. 10, 1918-1928 (2011).

74. Tarragó-Trani M. T., Jiang S., Harich K. C. \& Storrie B. Shiga-like toxin subunit B (SLTB)-enhanced delivery of chlorin e6 (Ce6) improves cell killing. Photochem. Photobiol. 82, 527-537 (2006). 
75. Ryou J.-H., Sohn Y.-K., Hwang D.-E. \& Kim H.-S. Shiga-like toxin-based high-efficiency and receptor-specific intracellular delivery system for a protein. Biochem. Biophys. Res. Commun. 464, 1282-1289 (2015).

76. Bouter A., Delord B., Dransart E., Poirier C., Johannes L. \& Van Effenterre D. Intracellular trafficking of Shiga-toxin-B-subunit-functionalized spherulites. Biol. Cell 100, 717-728 (2008).

77. Singer S. J. \& Nicolson G. L. The fluid mosaic model of the structure of cell membranes. Science 175, 720-731 (1972).

78. Van Meer G. \& de Kroon A. I. Lipid map of the mammalian cell. J. Cell Sci. 124, 5-8 (2011).

79. Harayama T. \& Riezman H. Understanding the diversity of membrane lipid composition. Nat. Rev. Mol. Cell Biol. 19, 281 (2018).

80. Simons K. \& Ikonen E. Functional rafts in cell membranes. Nature 387, 569572 (1997).

81. Goñi F. M. "Rafts": a nickname for putative transient nanodomains. Chem. Phys. Lipids 218, 34-39 (2019).

82. Cebecauer M., Amaro M., Jurkiewicz P., Sarmento M. J., Šachl R., Cwiklik L. \& Hof M. Membrane Lipid Nanodomains. Chem. Rev. 118, 11259-11297 (2018).

83. Raghunathan K. \& Kenworthy A. K. Dynamic pattern generation in cell membranes: Current insights into membrane organization. Biochim. Biophys. Acta, Biomembr. 1860, 2018-2031 (2018).

84. Pike L. J. Rafts defined: a report on the Keystone Symposium on Lipid Rafts and Cell Function. J. Lipid Res. 47, 1597-1598 (2006).

85. Sezgin E., Levental I., Mayor S. \& Eggeling C. The mystery of membrane organization: composition, regulation and roles of lipid rafts. Nat. Rev. Mol. Cell Biol. 18, 361 (2017).

86. Cheng X. \& Smith J. C. Biological Membrane Organization and Cellular Signaling. Chem. Rev. 119, 5849-5880 (2019).

87. Liu A. P. \& Fletcher D. A. Actin polymerization serves as a membrane domain switch in model lipid bilayers. Biophys. J. 91, 4064-4070 (2006).

88. Marushchak D., Gretskaya N., Mikhalyov I. \& Johansson L. B.-Å. Self-aggregation-an intrinsic property of GM1 in lipid bilayers. Mol. Membr. Biol. 24, 102-112 (2007). 
89. Van Zanten T. S. \& Mayor S. Current approaches to studying membrane organization. F1000Res. 4 (2015).

90. Brown D. A. \& London E. Structure and function of sphingolipid-and cholesterolrich membrane rafts. J. Biol. Chem. 275, 17221-17224 (2000).

91. Rao M. \& Mayor S. Use of Forster's resonance energy transfer microscopy to study lipid rafts. Biochim. Biophys. Acta, Mol. Cell Res. 1746, 221-233 (2005).

92. Loura L. M., Fedorov A. \& Prieto M. Fluid-fluid membrane microheterogeneity: a fluorescence resonance energy transfer study. Biophys. J. 80, 776-788 (2001).

93. Krieger J. W., Singh A. P., Bag N., Garbe C. S., Saunders T. E., Langowski J. \& Wohland T. Imaging fluorescence (cross-) correlation spectroscopy in live cells and organisms. Nat. Protoc. 10, 1948 (2015).

94. Bag N., Ng X. W., Sankaran J. \& Wohland T. Spatiotemporal mapping of diffusion dynamics and organization in plasma membranes. Method. Appl. Fluoresc. 4, 034003 (2016).

95. Nickels J. D., Chatterjee S., Stanley C. B., Qian S., Cheng X., Myles D. A., Standaert R. F., Elkins J. G. \& Katsaras J. The in vivo structure of biological membranes and evidence for lipid domains. PLoS Biol. 15, e2002214 (2017).

96. Bernardino de la Serna J., Schütz G. J., Eggeling C. \& Cebecauer M. There is no simple model of the plasma membrane organization. Front. Cell Dev. Biol. 4, 106 (2016).

97. Feigenson G. W. Phase diagrams and lipid domains in multicomponent lipid bilayer mixtures. Biochim. Biophys. Acta, Biomembr. 1788, 47-52 (2009).

98. London E. How principles of domain formation in model membranes may explain ambiguities concerning lipid raft formation in cells. Biochim. Biophys. Acta, Mol. Cell Res. 1746, 203-220 (2005).

99. Veatch S. L. \& Keller S. L. Miscibility phase diagrams of giant vesicles containing sphingomyelin. Phys. Rev. Lett. 94, 148101 (2005).

100. Dimova R. Giant vesicles and their use in assays for assessing membrane phase state, curvature, mechanics, and electrical properties. Annu. Rev. Biophys. 48, 93-119 (2019).

101. Aoki P., Schroder A., Constantino C. \& Marques C. Bioadhesive giant vesicles for monitoring hydroperoxidation in lipid membranes. Soft Matter 11, 59955998 (2015). 
102. Janshoff A. \& Steinem C. Mechanics of lipid bilayers: What do we learn from pore-spanning membranes? Biochim. Biophys. Acta, Mol. Cell Res. 1853, 2977-2983 (2015).

103. Chan Y.-H. M. \& Boxer S. G. Model membrane systems and their applications. Curr. Opin. Chem. Biol. 11, 581-587 (2007).

104. Kubsch B., Robinson T., Lipowsky R. \& Dimova R. Solution asymmetry and salt expand fluid-fluid coexistence regions of charged membranes. Biophys. J. 110, 2581-2584 (2016).

105. Kubsch B., Robinson T., Steinkühler J. \& Dimova R. Phase Behavior of Charged Vesicles Under Symmetric and Asymmetric Solution Conditions Monitored with Fluorescence Microscopy. J. Vis. Exp. 128, e56034 (2017).

106. Diaz A. J., Albertorio F., Daniel S. \& Cremer P. S. Double cushions preserve transmembrane protein mobility in supported bilayer systems. Langmuir 24, 6820-6826 (2008).

107. Tanaka M. \& Sackmann E. Polymer-supported membranes as models of the cell surface. Nature 437, 656 (2005).

108. Sheetz M. P. Cell control by membrane-cytoskeleton adhesion. Nat. Rev. Mol. Cell Biol. 2, 392-396 (2001).

109. Nambiar R., McConnell R. E. \& Tyska M. J. Control of cell membrane tension by myosin-I. Proc. Natl. Acad. Sci. U.S.A. 106, 11972-11977 (2009).

110. Gleisner M., Kroppen B., Fricke C., Teske N., Kliesch T.-T., Janshoff A., Meinecke M. \& Steinem C. Epsin N-terminal homology domain (ENTH) activity as a function of membrane tension. J. Biol. Chem. 291, 19953-19961 (2016).

111. Van Dijck P., De Kruijff B., Van Deenen L., De Gier J. \& Demel R. The preference of cholesterol for phosphatidylcholine in mixed phosphatidylcholinephosphatidylethanolamine bilayers. Biochim. Biophys. Acta, Biomembr. 455, 576-587 (1976).

112. Ulrich A. S., Sami M. \& Watts A. Hydration of DOPC bilayers by differential scanning calorimetry. Biochim. Biophys. Acta, Biomembr. 1191, 225-230 (1994). 
113. Lewis R. N., Sykes B. D. \& McElhaney R. N. Thermotropic phase behavior of model membranes composed of phosphatidylcholines containing cis-monounsaturated acyl chain homologs of oleic acid: differential scanning calorimetric and phosphorus-31 NMR spectroscopic studies. Biochemistry 27, 880-887 (1988).

114. Koynova R. \& Caffrey M. Phases and phase transitions of the phosphatidylcholines. Biochim. Biophys. Acta, Rev. Biomembr. 1376, 91-145 (1998).

115. Lynch D. V. \& Steponkus P. L. Lyotropic phase behavior of unsaturated phosphatidylcholine species: relevance to the mechanism of plasma membrane destabilization and freezing injury. Biochim. Biophys. Acta, Biomembr. 984, 267272 (1989).

116. Coolbear K., Berde C. \& Keough K. Gel to liquid-crystalline phase transitions of aqueous dispersions of polyunsaturated mixed-acid phosphatidylcholines. Biochemistry 22, 1466-1473 (1983).

117. Barton P. G. \& Gunstone F. Hydrocarbon chain packing and molecular motion in phospholipid bilayers formed from unsaturated lecithins. Synthesis and properties of sixteen positional isomers of 1, 2-dioctadecenoyl-sn-glycero-3phosphorylcholine. J. Biol. Chem. 250, 4470-4476 (1975).

118. Ladbrooke B. \& Chapman D. Thermal analysis of lipids, proteins and biological membranes a review and summary of some recent studies. Chem. Phys. Lipids 3, 304-356 (1969).

119. Ipsen J., Mouritsen O. \& Bloom M. Relationships between lipid membrane area, hydrophobic thickness, and acyl-chain orientational order. The effects of cholesterol. Biophys. J. 57, 405-412 (1990).

120. Shaw K. P., Brooks N. J., Clarke J. A., Ces O., Seddon J. M. \& Law R. V. Pressure-temperature phase behaviour of natural sphingomyelin extracts. Soft Matter 8, 1070-1078 (2012).

121. Byrdwell W. C. \& Perry R. H. Liquid chromatography with dual parallel mass spectrometry and 31P nuclear magnetic resonance spectroscopy for analysis of sphingomyelin and dihydrosphingomyelin: I. Bovine brain and chicken egg yolk. J. Chromatogr., A 1133, 149-171 (2006).

122. Koynova R. \& Caffrey M. Phases and phase transitions of the sphingolipids. Biochim. Biophys. Acta, Lipids Lipid Metab. 1255, 213-236 (1995). 
123. Kawasaki Y., Nishikido H., Kuboki A., Ohira S. \& Kodama M. Unilamellar vesicle-forming property of N-nervonoylsphingomyelin (C24: 1-SM) as studied by differential scanning calorimetry and negative stain electron microscopy. Thermochim. Acta 431, 188-194 (2005).

124. Aoki H., Kosakabe S., Inumaru M., Kuboki A., Ohira S. \& Kodama M. Structural property and function of D-erythro asymmetric chain sphingomyelins as studied by microcalorimetry and electron microscopy. J. Therm. Anal. Calorim. 92, 443 (2008).

125. Kodama M. \& Kawasaki Y. Structural role of mismatched $\mathrm{C}-\mathrm{C}$ bonds in a series of d-erythro-sphingomyelins as studied by DSC and electron microscopy. Chem. Phys. Lipids 163, 514-523 (2010).

126. Kodama M., Kawasaki Y. \& Ohtaka H. The main transition enthalpy of the gel-to-liquid crystal phases for a series of asymmetric chain length D-erythro (2S, 3R) sphingomyelins. Thermochim. Acta 532, 22-27 (2012).

127. Sripada P., Maulik P., Hamilton J. \& Shipley G. Partial synthesis and properties of a series of N-acyl sphingomyelins. J. Lipid Res. 28, 710-718 (1987).

128. Björkqvist Y. J. E., Brewer J., Bagatolli L. A., Slotte J. P. \& Westerlund B. Thermotropic behavior and lateral distribution of very long chain sphingolipids. Biochim. Biophys. Acta, Biomembr. 1788, 1310-1320 (2009).

129. Calhoun W. I. \& Shipley G. G. Sphingomyelin-lecithin bilayers and their interaction with cholesterol. Biochemistry 18, 1717-1722 (1979).

130. Barenholz Y., Suurkuusk J., Mountcastle D., Thompson T. \& Biltonen R. A calorimetric study of the thermotropic behavior of aqueous dispersions of natural and synthetic sphingomyelins. Biochemistry 15, 2441-2447 (1976).

131. Li X.-M., Smaby J. M., Momsen M. M., Brockman H. L. \& Brown R. E. Sphingomyelin interfacial behavior: the impact of changing acyl chain composition. Biophys. J. 78, 1921-1931 (2000).

132. Cohen R., Barenholz Y., Gatt S. \& Dagan A. Preparation and characterization of well defined D-erythro sphingomyelins. Chem. Phys. Lipids 35, 371-384 (1984).

133. Jimenez-Rojo N., Garcia-Arribas A. B., Sot J., Alonso A. \& Goni F. M. Lipid bilayers containing sphingomyelins and ceramides of varying N-acyl lengths: a glimpse into sphingolipid complexity. Biochim. Biophys. Acta, Biomembr. 1838, 456-464 (2014). 
134. Jaikishan S., Björkbom A. \& Slotte J. P. Sphingomyelin analogs with branched N-acyl chains: the position of branching dramatically affects acyl chain order and sterol interactions in bilayer membranes. Biochim. Biophys. Acta, Biomembr. 1798, 1987-1994 (2010).

135. Epand R. M. \& Moscarello M. A. The effect of bovine myelin basic protein on the phase transition properties of sphingomyelin. Biochim. Biophys. Acta, Biomembr. 685, 230-232 (1982).

136. Ohtaka H., Kawasaki Y. \& Kodama M. Phase transitions of highly asymmetric chain-length N-lignocerylsphingomyelin (C24: 0-SM) bilayer. J. Therm. Anal. Calorim. 113, 1593-1602 (2013).

137. Maulik P. R. \& Shipley G. G. X-ray diffraction and calorimetric study of Nlignoceryl sphingomyelin membranes. Biophys. J. 69, 1909-1916 (1995).

138. McIntosh T. J., Simon S. A., Needham D. \& Huang C. H. Structure and cohesive properties of sphingomyelin/cholesterol bilayers. Biochemistry 31, 20122020 (1992).

139. Baumgart T., Hunt G., Farkas E. R., Webb W. W. \& Feigenson G. W. Fluorescence probe partitioning between $\mathrm{L}_{\mathrm{o}} / \mathrm{L}_{\mathrm{d}}$ phases in lipid membranes. Biochim. Biophys. Acta, Biomembr. 1768, 2182-2194 (2007).

140. Klymchenko A. S. \& Kreder R. Fluorescent probes for lipid rafts: from model membranes to living cells. Chem. Biol. 21, 97-113 (2014).

141. Patalag L. J., Sibold J., Schütte O. M., Steinem C. \& Werz D. B. Gb3 Glycosphingolipids with Fluorescent Oligoene Fatty Acids: Synthesis and Phase Behavior in Model Membranes. ChemBioChem 18, 2171-2178 (2017).

142. Sezgin E., Levental I., Grzybek M., Schwarzmann G., Mueller V., Honigmann A., Belov V. N., Eggeling C., Coskun Ü., Simons K. \& Schwille P. Partitioning, diffusion, and ligand binding of raft lipid analogs in model and cellular plasma membranes. Biochim. Biophys. Acta, Biomembr. 1818, 1777-1784 (2012).

143. Sibold J., Kettelhoit K., Vuong L., Liu F., Werz D. B. \& Steinem C. Synthesis of Head Group Labeled Gb3 Glycosphingolipids and Their Distribution in PhaseSeparated Giant Unilamellar Vesicles. Angew. Chem. Int. Ed. 58, 17805-17813 (2019).

144. Johannes L., Tenza D., Antony C. \& Goud B. Retrograde transport of KDELbearing B-fragment of Shiga toxin. J. Biol. Chem. 272, 19554-19561 (1997). 
145. Falguières T., Römer W., Amessou M., Afonso C., Wolf C., Tabet J.-C., Lamaze C. \& Johannes L. Functionally different pools of Shiga toxin receptor, globotriaosyl ceramide, in HeLa cells. FEBS J. 273, 5205-5218 (2006).

146. Bosse M., Sibold J., Scheidt H. A., Patalag L. J., Kettelhoit K., Ries A., Werz D. B., Steinem C. \& Huster D. Shiga Toxin Binding Alters Lipid Packing and Domain Structure of $\mathrm{Gb}_{3}$-Containing Membranes: A Solid-State NMR Study. Phys. Chem. Chem. Phys. 21, 15630-15638 (2019).

147. Spindler S., Sibold J., Gholami Mahmoodabadi R., Steinem C. \& Sandoghdar V. High-Speed Microscopy of Diffusion in Pore-Spanning Lipid Membranes. Nano Lett. 18, 5262-5271 (2018).

148. Teske N., Sibold J., Schumacher J., Teiwes N. K., Gleisner M., Mey I. \& Steinem C. Continuous Pore-Spanning Lipid Bilayers On Silicon Oxide-Coated Porous Substrates. Langmuir 33, 14175-14183 (2017).

149. Klostermeier D. \& Rudolph M. G. Biophysical Chemistry 1. Aufl. ISBN: 978-1482-25224-8 (CRC Press, Boca Raton, Fla, 2018).

150. Benda A., Beneš M., Marecek V., Lhotskỳ A., Hermens W. T. \& Hof M. How to determine diffusion coefficients in planar phospholipid systems by confocal fluorescence correlation spectroscopy. Langmuir 19, 4120-4126 (2003).

151. Lakowicz J. R. Principles of fluorescence spectroscopy 3rd ed. 954 pp. ISBN: 978-0-387-31278-1 (Springer, New York, 2006).

152. Geddes C. D. Reviews in Fluorescence 2009 (Springer Science \& Business Media, 2011).

153. Wohland T., Rigler R. \& Vogel H. The standard deviation in fluorescence correlation spectroscopy. Biophys. J. 80, 2987-2999 (2001).

154. Vestergaard C. L., Blainey P. C. \& Flyvbjerg H. Optimal estimation of diffusion coefficients from single-particle trajectories. Phys. Rev. E: Stat., Nonlinear, Soft Matter Phys. 89, 022726 (2014).

155. Nehls S. \& Janshoff A. Elastic Properties of Pore-Spanning Apical Cell Membranes Derived from MDCK II Cells. Biophys. J. 113, 1822-1830 (2017).

156. Schwamborn M., Schumacher J., Sibold J., Teiwes N. K. \& Steinem C. Monitoring ATPase induced $\mathrm{pH}$ changes in single proteoliposomes with the lipidcoupled fluorophore Oregon Green 488. Analyst 142, 2670-2677 (2017). 
157. Fuchs G., Mobassaleh M., Donohue-Rolfe A., Montgomery R., Grand R. \& Keusch G. Pathogenesis of Shigella diarrhea: rabbit intestinal cell microvillus membrane binding site for Shigella toxin. Infect. Immun. 53, 372-377 (1986).

158. Eiklid K. \& Olsnes S. Interaction of Shigella shigae cytotoxin with receptors on sensitive and insensitive cells. J. Receptor Res. 1, 199-213 (1980).

159. Pina D. G. \& Johannes L. Cholera and Shiga toxin B-subunits: thermodynamic and structural considerations for function and biomedical applications. Toxicon 45, 389-393 (2005).

160. Schütte O. M., Patalag L. J., Weber L. M., Ries A., Römer W., Werz D. B. \& Steinem C. 2-Hydroxy Fatty Acid Enantiomers of Gb 3 Impact Shiga Toxin Binding and Membrane Organization. Biophys. J. 108, 2775-2778 (2015).

161. Head S. C., Karmali M. A. \& Lingwood C. A. Preparation of VT1 and VT2 hybrid toxins from their purified dissociated subunits. Evidence for B subunit modulation of a subunit function. J. Biol. Chem. 266, 3617-3621 (1991).

162. Schütte O. M., Ries A., Orth A., Patalag L. J., Römer W., Steinem C. \& Werz D. B. Influence of Gb 3 glycosphingolipids differing in their fatty acid chain on the phase behaviour of solid supported membranes: chemical syntheses and impact of Shiga toxin binding. Chem. Sci. 5, 3104-3114 (2014).

163. Castanho M., Coutinho A. \& Prieto M. Absorption and fluorescence spectra of polyene antibiotics in the presence of cholesterol. J. Biol. Chem. 267, 204-209 (1992).

164. Nieves I., Artetxe I., Abad J. L., Alonso A., Busto J. V., Fajarí L., Montes L. R., Sot J., Delgado A. \& Goñi F. M. Fluorescent Polyene Ceramide Analogues as Membrane Probes. Langmuir 31, 2484-2492 (2015).

165. Johnson I. D., Kang H. C. \& Haugland R. P. Fluorescent membrane probes incorporating dipyrrometheneboron difluoride fluorophores. Anal. Biochem. 198, 228-237 (1991).

166. Boldyrev I. A., Zhai X., Momsen M. M., Brockman H. L., Brown R. E. \& Molotkovsky J. G. New BODIPY lipid probes for fluorescence studies of membranes. J. Lipid Res. 48, 1518-1532 (2007).

167. Kaiser R. D. \& London E. Determination of the depth of BODIPY probes in model membranes by parallax analysis of fluorescence quenching. Biochim. Biophys. Acta, Biomembr. 1375, 13-22 (1998). 
168. Karolin J., Johansson L. B.-A., Strandberg L. \& Ny T. Fluorescence and absorption spectroscopic properties of dipyrrometheneboron difluoride (BODIPY) derivatives in liquids, lipid membranes, and proteins. J. Am. Chem. Soc. 116, 7801-7806 (1994).

169. Mikhalyov I., Gretskaya N. \& Johansson L. B.-Å. Fluorescent BODIPY-labelled G M1 gangliosides designed for exploring lipid membrane properties and specific membrane-target interactions. Chem. Phys. Lipids 159, 38-44 (2009).

170. Ariola F. S., Li Z., Cornejo C., Bittman R. \& Heikal A. A. Membrane fluidity and lipid order in ternary giant unilamellar vesicles using a new bodipycholesterol derivative. Biophys. J. 96, 2696-2708 (2009).

171. O'Connor D., Byrne A. \& Keyes T. E. Linker length in fluorophore-cholesterol conjugates directs phase selectivity and cellular localisation in GUVs and live cells. RSC Adv. 9, 22805-22816 (2019).

172. Collot M., Boutant E., Lehmann M. \& Klymchenko A. S. BODIPY with Tuned Amphiphilicity as a Fluorogenic Plasma Membrane Probe. Bioconjugate Chem. 30, 192-199 (2018).

173. MacDonald R. I. Characteristics of self-quenching of the fluorescence of lipidconjugated rhodamine in membranes. J. Biol. Chem. 265, 13533-13539 (1990).

174. London E. \& Brown D. A. Insolubility of lipids in triton X-100: physical origin and relationship to sphingolipid/cholesterol membrane domains (rafts). Biochim. Biophys. Acta, Biomembr. 1508, 182-195 (2000).

175. Lingwood D. \& Simons K. Detergent resistance as a tool in membrane research. Nat. Protoc. 2, 2159 (2007).

176. Manni M. M., Cano A., Alonso C. \& Goñi F. M. Lipids that determine detergent resistance of MDCK cell membrane fractions. Chem. Phys. Lipids 191, 68-74 (2015).

177. Komura N., Suzuki K. G., Ando H., Konishi M., Koikeda M., Imamura A., Chadda R., Fujiwara T. K., Tsuboi H., Sheng R., et al. Raft-based interactions of gangliosides with a GPI-anchored receptor. Nat. Chem. Biol. (2016).

178. Sengupta P., Hammond A., Holowka D. \& Baird B. Structural determinants for partitioning of lipids and proteins between coexisting fluid phases in giant plasma membrane vesicles. Biochim. Biophys. Acta, Biomembr. 1778, 20-32 (2008). 
179. Bacia K., Schwille P. \& Kurzchalia T. Sterol structure determines the separation of phases and the curvature of the liquid-ordered phase in model membranes. Proc. Natl. Acad. Sci. U.S.A. 102, 3272-3277 (2005).

180. Rissanen S., Grzybek M., Orłowski A., Róg T., Cramariuc O., Levental I., Eggeling C., Sezgin E. \& Vattulainen I. Phase Partitioning of GM1 and Its Bodipy-Labeled Analog Determine Their Different Binding to Cholera Toxin. Front. Physiol. 8, 252 (2017).

181. Kahya N., Brown D. A. \& Schwille P. Raft partitioning and dynamic behavior of human placental alkaline phosphatase in giant unilamellar vesicles. Biochemistry 44, 7479-7489 (2005).

182. Hammond A., Heberle F., Baumgart T., Holowka D., Baird B. \& Feigenson G. Crosslinking a lipid raft component triggers liquid ordered-liquid disordered phase separation in model plasma membranes. Proc. Natl. Acad. Sci. U.S.A. 102, 6320-6325 (2005).

183. Kitov P. I. \& Bundle D. R. Synthesis and structure-activity relationships of diand trisaccharide inhibitors for Shiga-like toxin Type 1. J. Chem. Soc., Perkin Trans. 1, 838-853 (2001).

184. Kitov P. I., Shimizu H., Homans S. W. \& Bundle D. R. Optimization of tether length in nonglycosidically linked bivalent ligands that target sites 2 and 1 of a Shiga-like toxin. J. Am. Chem. Soc. 125, 3284-3294 (2003).

185. Römer W., Pontani L.-L., Sorre B., Rentero C., Berland L., Chambon V., Lamaze C., Bassereau P., Sykes C. \& Gaus K. Actin dynamics drive membrane reorganization and scission in clathrin-independent endocytosis. Cell 140, 540$553(2010)$.

186. Smith D. C., Sillence D. J., Falguieres T., Jarvis R. M., Johannes L., Lord J. M., Platt F. M. \& Roberts L. M. The association of Shiga-like toxin with detergent-resistant membranes is modulated by glucosylceramide and is an essential requirement in the endoplasmic reticulum for a cytotoxic effect. Mol. Biol. Cell 17, 1375-1387 (2006).

187. Raa H., Grimmer S., Schwudke D., Bergan J., Wälchli S., Skotland T., Shevchenko A. \& Sandvig K. Glycosphingolipid requirements for endosome-to-Golgi transport of Shiga toxin. Traffic 10, 868-882 (2009). 
188. Honigmann A., Mueller V., Hell S. W. \& Eggeling C. STED microscopy detects and quantifies liquid phase separation in lipid membranes using a new far-red emitting fluorescent phosphoglycerolipid analogue. Faraday Discuss. 161, 7789 (2013).

189. Bordovsky S. S., Wong C. S., Bachand G. D., Stachowiak J. C. \& Sasaki D. Y. Engineering lipid structure for recognition of the liquid ordered membrane phase. Langmuir 32, 12527-12533 (2016).

190. Momin N., Lee S., Gadok A. K., Busch D. J., Bachand G. D., Hayden C. C., Stachowiak J. C. \& Sasaki D. Y. Designing lipids for selective partitioning into liquid ordered membrane domains. Soft Matter 11, 3241-3250 (2015).

191. Stefaniu C., Ries A., Gutowski O., Ruett U., Seeberger P. H., Werz D. B. \& Brezesinski G. Impact of Structural Differences in Galactocerebrosides on the Behavior of 2D Monolayers. Langmuir 32, 2436-2444 (2016).

192. Blume A. A comparative study of the phase transitions of phospholipid bilayers and monolayers. Biochim. Biophys. Acta, Biomembr. 557, 32-44 (1979).

193. Morrow M. R., Singh D. M. \& Grant C. W. Glycosphingolipid acyl chain order profiles: substituent effects. Biochim. Biophys. Acta, Biomembr. 1235, 239248 (1995).

194. Sáenz J. P., Sezgin E., Schwille P. \& Simons K. Functional convergence of hopanoids and sterols in membrane ordering. Proc. Natl. Acad. Sci. U.S.A. 109, 14236-14240 (2012).

195. Maté S., Busto J. V., García-Arribas A. B., Sot J., Vazquez R., Herlax V., Wolf C., Bakás L. \& Goni F. M. N-Nervonoylsphingomyelin (c24: 1) prevents lateral heterogeneity in cholesterol-containing membranes. Biophys. J. 106, 2606-2616 (2014).

196. Morrow M. R., Singh D. M. \& Grant C. W. Glycosphingolipid headgroup orientation in fluid phospholipid/cholesterol membranes: similarity for a range of glycolipid fatty acids. Biophys. J. 69, 955-964 (1995).

197. Ekholm O., Jaikishan S., Lönnfors M., Nyholm T. K. \& Slotte J. P. Membrane bilayer properties of sphingomyelins with amide-linked 2-or 3-hydroxylated fatty acids. Biochim. Biophys. Acta, Biomembr. 1808, 727-732 (2011).

198. Lingwood D., Binnington B., Róg T., Vattulainen I., Grzybek M., Coskun Ü., Lingwood C. A. \& Simons K. Cholesterol modulates glycolipid conformation and receptor activity. Nat. Chem. Biol. 7, 260-262 (2011). 
199. Yahi N., Aulas A. \& Fantini J. How Cholesterol Constrains Glycolipid Conformation for Optimal Recognition of Alzheimer's $\beta$ Amyloid Peptide (A $\beta$ 1-40). PLoS One 5, e9079 (2010).

200. Balleza D., Mescola A., Marín-Medina N., Ragazzini G., Pieruccini M., Facci P. \& Alessandrini A. Complex phase behavior of GUVs containing different Sphingomyelins. Biophys. J. 116, 503-517 (2019).

201. Marsh D. Handbook of lipid bilayers (CRC press, 2013).

202. Ramstedt B. \& Slotte J. P. Interaction of cholesterol with sphingomyelins and acyl-chain-matched phosphatidylcholines: a comparative study of the effect of the chain length. Biophys. J. 76, 908-915 (1999).

203. Niemelä P. S., Hyvönen M. T. \& Vattulainen I. Influence of chain length and unsaturation on sphingomyelin bilayers. Biophys. J. 90, 851-863 (2006).

204. Maulik P., Sripada P. \& Shipley G. Structure and thermotropic properties of hydrated N-stearoyl sphingomyelin bilayer membranes. Biochim. Biophys. Acta, Biomembr. 1062, 211-219 (1991).

205. Maulik P., Atkinson D. \& Shipley G. X-ray scattering of vesicles of N-acyl sphingomyelins. Determination of bilayer thickness. Biophys. J. 50, 1071-1077 (1986).

206. Takahashi H., Hayakawa T., Kawasaki Y., Ito K., Fujisawa T., Kodama M. \& Kobayashi T. Structural characterization of N-lignoceroyl (C24: 0) sphingomyelin bilayer membranes: a re-evaluation. Appl. Crystallogr. 40, s312-s317 (2007).

207. Róg T., Orłowski A., Llorente A., Skotland T., Sylvänne T., Kauhanen D., Ekroos K., Sandvig K. \& Vattulainen I. Interdigitation of long-chain sphingomyelin induces coupling of membrane leaflets in a cholesterol dependent manner. Biochim. Biophys. Acta, Biomembr. 1858, 281-288 (2016).

208. Jaikishan S. \& Slotte J. P. Effect of hydrophobic mismatch and interdigitation on sterol/sphingomyelin interaction in ternary bilayer membranes. Biochim. Biophys. Acta, Biomembr. 1808, 1940-1945 (2011).

209. Orth A., Johannes L., Römer W. \& Steinem C. Creating and Modulating Microdomains in Pore-Spanning Membranes. ChemPhysChem 13, 108-114 (2012).

210. Hasegawa T. in Glycobiophysics 21-39 (Springer, 2018). 
211. Todeschini A. R. \& Hakomori S.-i. Functional role of glycosphingolipids and gangliosides in control of cell adhesion, motility, and growth, through glycosynaptic microdomains. Biochim. Biophys. Acta, Gen. Subj. 1780, 421-433 (2008).

212. Missler M., Südhof T. C. \& Biederer T. Synaptic cell adhesion. Cold Spring Harb.Perspect.Biol. 4, a005694 (2012).

213. Honigmann A., Sadeghi S., Keller J., Hell S. W., Eggeling C. \& Vink R. A lipid bound actin meshwork organizes liquid phase separation in model membranes. eLife 3, e01671 (2014).

214. Kuhlmann J. W., Mey I. P. \& Steinem C. Modulating the lateral tension of solvent-free pore-spanning membranes. Langmuir 30, 8186-8192 (2014).

215. Dai J. \& Sheetz M. P. Membrane tether formation from blebbing cells. Biophys. J. 77, 3363-3370 (1999).

216. Lieber A. D., Yehudai-Resheff S., Barnhart E. L., Theriot J. A. \& Keren K. Membrane tension in rapidly moving cells is determined by cytoskeletal forces. Curr. Biol. 23, 1409-1417 (2013).

217. Reineck P., Gómez D., Ng S. H., Karg M., Bell T., Mulvaney P. \& Bach U. Distance and wavelength dependent quenching of molecular fluorescence by Au@ SiO2 core-shell nanoparticles. ACS Nano 7, 6636-6648 (2013).

218. Dulkeith E., Morteani A., Niedereichholz T., Klar T., Feldmann J., Levi S., Van Veggel F., Reinhoudt D., Möller M. \& Gittins D. Fluorescence quenching of dye molecules near gold nanoparticles: radiative and nonradiative effects. Phys. Rev. Lett. 89, 203002 (2002).

219. Dulkeith E., Ringler M., Klar T., Feldmann J., Munoz Javier A. \& Parak W. Gold nanoparticles quench fluorescence by phase induced radiative rate suppression. Nano Lett. 5, 585-589 (2005).

220. Kennedy G., Buiu O. \& Taylor S. Oxidation of silicon nitride films in an oxygen plasma. J. Appl. Phys. 85, 3319-3326 (1999).

221. Hair M. L. Hydroxyl groups on silica surface. J. Non-Cryst. Solids 19, 299-309 (1975).

222. Jennings T., Singh M. \& Strouse G. Fluorescent lifetime quenching near $d=$ $1.5 \mathrm{~nm}$ gold nanoparticles: probing NSET validity. J. Am. Chem. Soc. 128, $5462-5467$ (2006). 
223. Kittredge K. W., Fox M. A. \& Whitesell J. K. Effect of alkyl chain length on the fluorescence of 9-alkylfluorenyl thiols as self-assembled monolayers on gold. J. Phys. Chem. B 105, 10594-10599 (2001).

224. Hiasa T. \& Onishi H. Mercaptohexanol assembled on gold: FM-AFM imaging in water. Colloid. Surface. A. 441, 149-154 (2014).

225. Fogarassy E., Slaoui A., Fuchs C. \& Regolini J. Rapid thermal oxidation of silicon monoxide. Appl. Phys. Lett. 51, 337-339 (1987).

226. Baumgärtel T., von Borczyskowski C. \& Graaf H. Fluorescence studies of Rhodamine 6G functionalized silicon oxide nanostructures. Nanotechnology 21, 475205 (2010).

227. Hayashi T., Castner T. \& Boyd R. W. Quenching of molecular fluorescence near the surface of a semiconductor. Chem. Phys. Lett. 94, 461-466 (1983).

228. Whitmore P., Alivisatos A. \& Harris C. Distance Dependence of Electronic Energy Transfer to Semiconductor Surfaces: n $3 \pi^{*}$ Pyrazine/GaAs (110). Phys. Rev. Lett. 50, 1092 (1983).

229. Sezgin E., Schneider F., Zilles V., Urbančič I., Garcia E., Waithe D., Klymchenko A. S. \& Eggeling C. Polarity-Sensitive Probes for Superresolution Stimulated Emission Depletion Microscopy. Biophys. J. 113, 1321-1330 (2017).

230. Schütte O. M., Mey I., Enderlein J., Savić F., Geil B., Janshoff A. \& Steinem C. Size and mobility of lipid domains tuned by geometrical constraints. Proc. Natl. Acad. Sci. U.S.A. 201704199 (2017).

231. Sumitomo K. \& Oshima A. Liquid-Ordered/Liquid-Crystalline Phase Separation at a Lipid Bilayer Suspended over Microwells. Langmuir 33, 13277-13283 (2017).

232. Kudelski A. Chemisorption of 2-mercaptoethanol on silver, copper, and gold: direct Raman evidence of acid-induced changes in adsorption/desorption equilibria. Langmuir 19, 3805-3813 (2003).

233. Liu J., Paddon-Row M. N. \& Gooding J. J. Heterogeneous electron-transfer kinetics for flavin adenine dinucleotide and ferrocene through alkanethiol mixed monolayers on gold electrodes. J. Phys. Chem. B 108, 8460-8466 (2004).

234. Kocun M., Lazzara T. D., Steinem C. \& Janshoff A. Preparation of solvent-free, pore-spanning lipid bilayers: modeling the low tension of plasma membranes. Langmuir 27, 7672-7680 (2011). 
235. Mey I., Stephan M., Schmitt E. K., Müller M. M., Ben Amar M., Steinem C. \& Janshoff A. Local membrane mechanics of pore-spanning bilayers. J. Am. Chem. Soc. 131, 7031-7039 (2009).

236. Rawicz W., Olbrich K., McIntosh T., Needham D. \& Evans E. Effect of chain length and unsaturation on elasticity of lipid bilayers. Biophys. J. 79, 328-339 (2000).

237. Rawicz W., Smith B., McIntosh T., Simon S. \& Evans E. Elasticity, strength, and water permeability of bilayers that contain raft microdomain-forming lipids. Biophys. J. 94, 4725-4736 (2008).

238. Bezlyepkina N., Gracià R., Shchelokovskyy P., Lipowsky R. \& Dimova R. Phase diagram and tie-line determination for the ternary mixture DOPC/eSM/cholesterol. Biophys. J. 104, 1456-1464 (2013).

239. Kocun M. \& Janshoff A. Pulling Tethers from Pore-Spanning Bilayers: Towards Simultaneous Determination of Local Bending Modulus and Lateral Tension of Membranes. Small 8, 847-851 (2012).

240. Fuhrmans M. \& Müller M. Mechanisms of vesicle spreading on surfaces: coarsegrained simulations. Langmuir 29, 4335-4349 (2013).

241. Hoopes M. I., Faller R. \& Longo M. L. Lipid domain depletion at small localized bends imposed by a step geometry. Langmuir 27, 2783-2788 (2011).

242. Yoon T.-Y., Jeong C., Lee S.-W., Kim J. H., Choi M. C., Kim S.-J., Kim M. W. \& Lee S.-D. Topographic control of lipid-raft reconstitution in model membranes. Nat. Mater. 5, 281-285 (2006).

243. Parthasarathy R., Yu C.-h. \& Groves J. T. Curvature-modulated phase separation in lipid bilayer membranes. Langmuir 22, 5095-5099 (2006).

244. Callan-Jones A., Sorre B. \& Bassereau P. Curvature-driven lipid sorting in biomembranes. Cold Spring Harb.Perspect.Biol. a004648 (2011).

245. Callan-Jones A. \& Bassereau P. Curvature-driven membrane lipid and protein distribution. Curr. Opin. Solid State Mater. Sci. 17, 143-150 (2013).

246. Gracià R. S., Bezlyepkina N., Knorr R. L., Lipowsky R. \& Dimova R. Effect of cholesterol on the rigidity of saturated and unsaturated membranes: fluctuation and electrodeformation analysis of giant vesicles. Soft Matter 6, 1472-1482 (2010). 
247. Sorre B., Callan-Jones A., Manneville J.-B., Nassoy P., Joanny J.-F., Prost J., Goud B. \& Bassereau P. Curvature-driven lipid sorting needs proximity to a demixing point and is aided by proteins. Proc. Natl. Acad. Sci. U.S.A. 106, 5622-5626 (2009).

248. Hu J., Weikl T. \& Lipowsky R. Vesicles with multiple membrane domains. Soft Matter 7, 6092-6102 (2011).

249. McIntosh T. J., Vidal A. \& Simon S. A. Sorting of lipids and transmembrane peptides between detergent-soluble bilayers and detergent-resistant rafts. Biophys. J. 85, 1656-1666 (2003).

250. Dimova R. Recent developments in the field of bending rigidity measurements on membranes. Adv. Colloid Interfac. 208, 225-234 (2014).

251. Baumgart T., Das S., Webb W. \& Jenkins J. Membrane elasticity in giant vesicles with fluid phase coexistence. Biophys. J. 89, 1067-1080 (2005).

252. Kollmitzer B., Heftberger P., Podgornik R., Nagle J. F. \& Pabst G. Bending rigidities and interdomain forces in membranes with coexisting lipid domains. Biophys. J. 108, 2833-2842 (2015).

253. Zhao Y., Das S. \& Du Q. Adhesion of multicomponent vesicle membranes. Phys. Rev. E: Stat., Nonlinear, Soft Matter Phys. 81, 041919 (2010).

254. Semrau S., Idema T., Holtzer L., Schmidt T. \& Storm C. Accurate determination of elastic parameters for multicomponent membranes. Phys. Rev. Lett. 100, 088101 (2008).

255. Vestergaard C. L. Optimizing experimental parameters for tracking of diffusing particles. Phys. Rev. E: Stat., Nonlinear, Soft Matter Phys. 94, 022401 (2016).

256. Muddana H. S., Gullapalli R. R., Manias E. \& Butler P. J. Atomistic simulation of lipid and DiI dynamics in membrane bilayers under tension. Phys. Chem. Chem. Phys. 13, 1368-1378 (2011).

257. Reddy A. S., Warshaviak D. T. \& Chachisvilis M. Effect of membrane tension on the physical properties of DOPC lipid bilayer membrane. Biochim. Biophys. Acta, Biomembr. 1818, 2271-2281 (2012).

258. Muddana H. S., Gullapalli R. R., Tabouillot T. \& Butler P. J. Physiological membrane tension causes an increase in lipid diffusion: a single molecule fluorescence study. Biophys. J. 96, 197a-198a (2009). 
259. Thoms V. L., Hormel T. T., Reyer M. A. \& Parthasarathy R. Tension Independence of Lipid Diffusion and Membrane Viscosity. Langmuir 33, 12510-12515 (2017).

260. Hsieh C.-L., Spindler S., Ehrig J. \& Sandoghdar V. Tracking single particles on supported lipid membranes: multimobility diffusion and nanoscopic confinement. J. Phys. Chem. B 118, 1545-1554 (2014).

261. Spindler S., Ehrig J., König K., Nowak T., Piliarik M., Stein H. E., Taylor R. W., Garanger E., Lecommandoux S., Alves I. D., et al. Visualization of lipids and proteins at high spatial and temporal resolution via interferometric scattering (iSCAT) microscopy. J. Phys. D: Appl. Phys. 49, 274002 (2016).

262. Liao Y.-H., Lin C.-H., Cheng C.-Y., Wong W. C., Juo J.-Y. \& Hsieh C.-L. Monovalent and Oriented Labeling of Gold Nanoprobes for the High-Resolution Tracking of a Single Membrane Molecule. ACS Nano 13, 10918-10928 (2019). accepted.

263. Carrer D. C., Schmidt A. W., Knölker H.-J. \& Schwille P. Membrane domaindisrupting effects of 4-substitued cholesterol derivatives. Langmuir 24, 88078812 (2008).

264. Kahya N., Scherfeld D., Bacia K., Poolman B. \& Schwille P. Probing lipid mobility of raft-exhibiting model membranes by fluorescence correlation spectroscopy. J. Biol. Chem. 278, 28109-28115 (2003).

265. Przybylo M., Sýkora J., Humpolíčková J., Benda A., Zan A. \& Hof M. Lipid diffusion in giant unilamellar vesicles is more than 2 times faster than in supported phospholipid bilayers under identical conditions. Langmuir 22, 90969099 (2006).

266. Schneider F., Waithe D., Clausen M. P., Galiani S., Koller T., Ozhan G., Eggeling C. \& Sezgin E. Diffusion of lipids and GPI-anchored proteins in actinfree plasma membrane vesicles measured by STED-FCS. Mol. Biol. Cell 28, 1507-1518 (2017).

267. Schwenen L. L., Hubrich R., Milovanovic D., Geil B., Yang J., Kros A., Jahn R. \& Steinem C. Resolving single membrane fusion events on planar pore-spanning membranes. Sci. Rep. 5 (2015).

268. Schütz G. J., Kada G., Pastushenko V. P. \& Schindler H. Properties of lipid microdomains in a muscle cell membrane visualized by single molecule microscopy. EMBO J. 19, 892-901 (2000). 
269. Feder T. J., Brust-Mascher I., Slattery J. P., Baird B. \& Webb W. W. Constrained diffusion or immobile fraction on cell surfaces: a new interpretation. Biophys. J. 70, 2767-2773 (1996).

270. Fujiwara T., Ritchie K., Murakoshi H., Jacobson K. \& Kusumi A. Phospholipids undergo hop diffusion in compartmentalized cell membrane. J. Cell Biol. 157, 1071-1082 (2002).

271. Eggeling C., Ringemann C., Medda R., Schwarzmann G., Sandhoff K., Polyakova S., Belov V. N., Hein B., von Middendorff C., Schönle A., et al. Direct observation of the nanoscale dynamics of membrane lipids in a living cell. Nature 457, 1159-1162 (2009).

272. Veatch S. L. \& Keller S. L. Seeing spots: complex phase behavior in simple membranes. Biochim. Biophys. Acta, Mol. Cell Res. 1746, 172-185 (2005).

273. Pokorny A., Yandek L. E., Elegbede A. I., Hinderliter A. \& Almeida P. F. Temperature and composition dependence of the interaction of $\delta$-lysin with ternary mixtures of sphingomyelin/cholesterol/POPC. Biophys. J. 91, 21842197 (2006).

274. Konyakhina T. M. \& Feigenson G. W. Phase diagram of a polyunsaturated lipid mixture: brain sphingomyelin/1-stearoyl-2-docosahexaenoyl-sn-glycero-3phosphocholine/cholesterol. Biochim. Biophys. Acta, Biomembr. 1858, 153161 (2016).

275. Kahya N., Scherfeld D., Bacia K. \& Schwille P. Lipid domain formation and dynamics in giant unilamellar vesicles explored by fluorescence correlation spectroscopy. J. Struct. Biol. 147, 77-89 (2004).

276. Veatch S. L. \& Keller S. L. A Closer Look at the Canonical 'Raft Mixture'in Model Membrane Studies. Biophys. J. 84, 725 (2003).

277. Baykal-Caglar E., Hassan-Zadeh E., Saremi B. \& Huang J. Preparation of giant unilamellar vesicles from damp lipid film for better lipid compositional uniformity. Biochim. Biophys. Acta, Biomembr. 1818, 2598-2604 (2012).

278. Huang J., Buboltz J. T. \& Feigenson G. W. Maximum solubility of cholesterol in phosphatidylcholine and phosphatidylethanolamine bilayers. Biochim. Biophys. Acta, Biomembr. 1417, 89-100 (1999).

279. Stevens M. M., Honerkamp-Smith A. R. \& Keller S. L. Solubility limits of cholesterol, lanosterol, ergosterol, stigmasterol, and $\beta$-sitosterol in electroformed lipid vesicles. Soft Matter 6, 5882-5890 (2010). 
280. Rouhiparkouhi T., Weikl T. R., Discher D. E. \& Lipowsky R. Adhesion-induced phase behavior of two-component membranes and vesicles. Int. J. Mol. Sci. 14, 2203-2229 (2013).

281. Petruzielo R. S., Heberle F. A., Drazba P., Katsaras J. \& Feigenson G. W. Phase behavior and domain size in sphingomyelin-containing lipid bilayers. Biochim. Biophys. Acta, Biomembr. 1828, 1302-1313 (2013).

282. Carravilla P., Nieva J. L., Goñi F. M., Requejo-Isidro J. \& Huarte N. Twophoton Laurdan studies of the ternary lipid mixture DOPC: SM: cholesterol reveal a single liquid phase at sphingomyelin: cholesterol ratios lower than 1. Langmuir 31, 2808-2817 (2015).

283. Aufderhorst-Roberts A., Chandra U. \& Connell S. D. Three-phase coexistence in lipid membranes. Biophys. J. 112, 313-324 (2017).

284. Kuhlmann J. W., Junius M., Diederichsen U. \& Steinem C. SNARE-Mediated Single-Vesicle Fusion Events with Supported and Freestanding Lipid Membranes. Biophys. J. 112, 2348-2356 (2017).

285. Oshima A., Nakashima H. \& Sumitomo K. Evaluation of lateral diffusion of lipids in continuous membrane between freestanding and supported areas by fluorescence recovery after photobleaching. Langmuir (2019).

286. Bacia K., Scherfeld D., Kahya N. \& Schwille P. Fluorescence correlation spectroscopy relates rafts in model and native membranes. Biophys. J. 87, 10341043 (2004).

287. Scherfeld D., Kahya N. \& Schwille P. Lipid dynamics and domain formation in model membranes composed of ternary mixtures of unsaturated and saturated phosphatidylcholines and cholesterol. Biophys. J. 85, 3758-3768 (2003).

288. Kahya N., Scherfeld D. \& Schwille P. Differential lipid packing abilities and dynamics in giant unilamellar vesicles composed of short-chain saturated glycerolphospholipids, sphingomyelin and cholesterol. Chem. Phys. Lipids 135, 169180 (2005).

289. Guo L., Har J. Y., Sankaran J., Hong Y., Kannan B. \& Wohland T. Molecular diffusion measurement in lipid bilayers over wide concentration ranges: a comparative study. ChemPhysChem 9, 721-728 (2008).

290. Levental I., Byfield F. J., Chowdhury P., Gai F., Baumgart T. \& Janmey P. A. Cholesterol-dependent phase separation in cell-derived giant plasma-membrane vesicles. Biochem. J. 424, 163-167 (2009). 
291. Dietrich C., Bagatolli L., Volovyk Z., Thompson N., Levi M., Jacobson K. \& Gratton E. Lipid rafts reconstituted in model membranes. Biophys. J. 80, 1417-1428 (2001).

292. Korlach J., Schwille P., Webb W. W. \& Feigenson G. W. Characterization of lipid bilayer phases by confocal microscopy and fluorescence correlation spectroscopy. Proc. Natl. Acad. Sci. U.S.A. 96, 8461-8466 (1999).

293. Solanko L. M., Honigmann A., Midtiby H. S., Lund F. W., Brewer J. R., Dekaris V., Bittman R., Eggeling C. \& Wüstner D. Membrane orientation and lateral diffusion of BODIPY-cholesterol as a function of probe structure. Biophys. J. 105, 2082-2092 (2013).

294. Wu H.-M., Lin Y.-H., Yen T.-C. \& Hsieh C.-L. Nanoscopic substructures of raft-mimetic liquid-ordered membrane domains revealed by high-speed singleparticle tracking. Sci. Rep. 6, 20542 (2016).

295. Ries J., Chiantia S. \& Schwille P. Accurate determination of membrane dynamics with line-scan FCS. Biophys. J. 96, 1999-2008 (2009).

296. Chiantia S., Kahya N. \& Schwille P. Raft domain reorganization driven by short-and long-chain ceramide: a combined AFM and FCS study. Langmuir 23, 7659-7665 (2007).

297. Schindelin J., Arganda-Carreras I., Frise E., Kaynig V., Longair M., Pietzsch T., Preibisch S., Rueden C., Saalfeld S., Schmid B., et al. Fiji: an open-source platform for biological-image analysis. Nat. Methods 9, 676 (2012).

298. Sibold J., Tewaag V., Vagedes T., Mey I. \& Steinem C. Phase separation induced by surface adhesion (2019). in preparation. 



\section{A. Appendix}

\section{A.1. List of figures}

1.1. Reported EHEC disease in Germany in the last five years. . . . . . . 1

1.2. Protein structure of STx. . . . . . . . . . . . . . . . 3

1.3. The uptake of STx into the cell. . . . . . . . . . . . . . . 4

1.4. A schematically draw of the cell membrane. . . . . . . . . . . . 6

1.5. Four different model membrane systems. . . . . . . . . . . . . . . 7

3.1. A SPR measurement. . . . . . . . . . . . . . . . . . . . . . . . . . . . 29

3.2. Example GUV slide to show the analysis of the labeled $G_{3} \mathrm{~s}$. . . . . . 31

3.3. The fluorophore marker distribution in the $l_{\mathrm{o}} / l_{\mathrm{d}}$ and $l_{\beta} / l_{\mathrm{d}}$ phase separation in PSMs. . . . . . . . . . . . . . . . . . . . . . . . . 33

4.1. Schägger gel of different STxB purification's. . . . . . . . . . . . . . 38

4.2. Adsorption isotherms of different STxB purification's. . . . . . . . . . 40

4.3. Excitation and emission spectra of labeled $\mathrm{Gb}_{3} \mathrm{~s}$. . . . . . . . . . . . . 42

4.4. Emission spectra of labeled $\mathrm{Gb}_{3} \mathrm{~s}$ with different $l_{\mathrm{d}}$ phase markers. . . 44

4.5. Self-quenching of labeled $\mathrm{Gb}_{3} \mathrm{~s}$. . . . . . . . . . . . . . . . . 44

4.6. Liquid ordered phase distribution of the different fatty acid labeled $\mathrm{Gb}_{3} \mathrm{~S}$ without STxB. . . . . . . . . . . . . . 46

4.7. Liquid ordered phase distribution of the different fatty acid labeled

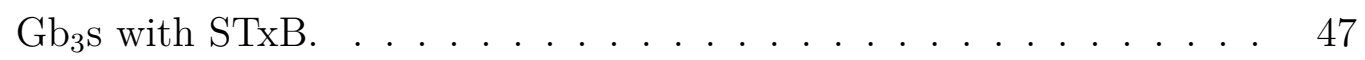

4.8. Visualization the binding of STxB to fatty acid labeled $\mathrm{Gb}_{3} \mathrm{~s}$. . . . . . 48

4.9. Phase distribution of head group labeled $\mathrm{Gb}_{3} \mathrm{~s}$ comparing the linker length. . . . . . . . . . . . . . . . . 51

4.10. Phase distribution of head group labeled $\mathrm{Gb}_{3} \mathrm{~s}$ comparing the saturation of the fatty acid. . . . . . . . . . . . . . . . . 53

4.11. Phase distribution of head group labeled $\mathrm{Gb}_{3} \mathrm{~s}$ comparing the hydroxilation of $\alpha$ position at the fatty acid. . . . . . . . . . . . . . . 54

4.12. Phase distribution of head group labeled $\mathrm{Gb}_{3} \mathrm{~s}$ with different sphingomyelins. . . . . . . . . . . . . . . . . 56 
4.13. Visualization the binding of STxB to head group labeled $\mathrm{Gb}_{3} \mathrm{~s}$. . . . . $\quad 59$

5.1. Model of the pore-spanning membrane. . . . . . . . . . . . . . . . . 63

5.2. SEM images of different orthogonality evaporated substrates. . . . . . 65

5.3. The different functionalizations for generating PSMs and there fluo-

rescent properties. . . . . . . . . . . . . . . 66 66

5.4. Phase separation on pore-spanning membrane (PSM) with the $\mathrm{SiO}_{\mathrm{x}}$ preparation. . . . . . . . . . . . . . . . . . . . 68

5.5. The binding of STxB on phase-separated PSMs with the $\mathrm{SiO}_{\mathrm{x}}$ functionalization. ......................... 70

5.6. The membrane tension of the pore-spanning membrane (PSM) from the gold mercaptoalcohol functionalization. . . . . . . . . . . . . . 72

5.7. The membrane tension of the pore-spanning membrane (PSM) from the $\mathrm{p}-\mathrm{Si}_{3} \mathrm{~N}_{4}$ and $\mathrm{SiO}_{\mathrm{x}}$ functionalization. . . . . . . . . . . . . . 74

5.8. The different Diffusion pathways of GNPs on PSMs . . . . . . . . . . 77

5.9. The different diffusion constant of GNPs on different functionalized PSMs. . . . . . . . . . . . . . . . . . . . . . . . 78

5.10. The different diffusion coefficient for the $l_{\mathrm{d}}$ and $l_{\mathrm{o}}$ phase on PSMs. . . 80

5.11. Fluorescence micrographs of phase-separated PSMs at $25^{\circ} \mathrm{C}$ and $55^{\circ} \mathrm{C}$. 83

5.12. Phase separation on PSM with Chol $8 \mathrm{~mol} \%$. . . . . . . . . . . 85

5.13. Phase separation on PSM with Chol $15 \mathrm{~mol} \%$. . . . . . . . . . . . 86

5.14. Phase separation on PSM with Chol $25 \mathrm{~mol} \%$. . . . . . . . . . . 87

5.15. The phase diagram in PSM with the lipid compositions DOPC/SM $\mathrm{Sorc}_{\text {p }} /$ Chol $(1 ; 1 ; \mathrm{x}) . \ldots \ldots \ldots$. . . . . . . . . . . . . . . 88

\section{A.2. List of schemes}

3.1. Structure of DOPC. . . . . . . . . . . . . . . . . . . . . . 11

3.2. Structure of Chol. . . . . . . . . . . . . . . . . . . . . . . . . 12

3.3. Structure of sphingomyelin. . . . . . . . . . . . . . . . 12

3.4. Structure of ATTO655-DOPE. . . . . . . . . . . . . . . . . . . . . 14

3.5. Structure of TexasRed-DHPE. . . . . . . . . . . . . . . . . . 14

3.6. Structure of ATTO655-DOPE. . . . . . . . . . . . . . . . . 15

3.7. Structure of Dy731-DOPE. . . . . . . . . . . . . . . . . . . . . . . . . . . . . . .

3.8. Structure of naphthopyrene. . . . . . . . . . . . . . . 16

3.9. Structure of BODIPY-Chol. . . . . . . . . . . . . . . . 16

3.10. Structure of $\mathrm{Gb}_{3}$. . . . . . . . . . . . . . . . . . . . . 17 


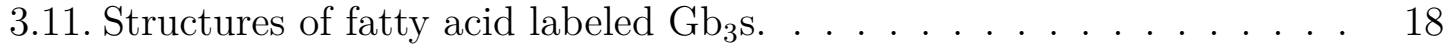

3.12. Structures of head group labeled $\mathrm{Gb}_{3} . \ldots \ldots$. . . . . . . . . . . . . 19

3.13. A process chart to calculate the different intensities of the PSMs. . . 35

3.14. A schematically draw of the $T_{\mathrm{M}}$ calcualtion. . . . . . . . . . . 36

A.1. All labeled $\mathrm{Gb}_{3} \mathrm{~S}$. . . . . . . . . . . . . . . . . . . . . . . . . . . 129

\section{A.3. List of tables}

3.1. SM distribution of $\mathrm{SM}_{\mathrm{porc}}$. . . . . . . . . . . . . . . . . . . 12

3.2. Sphingomyelins and their phase transition temperature. . . . . . . . . 13

4.1. $K_{\mathrm{d}}$ for the different STxB purification's. . . . . . . . . . . . . . . 39

4.2. Liquid ordered phase distribution of the different fatty acid labeled $\mathrm{Gb}_{3} \mathrm{~s}$. 47

4.3. Liquid ordered phase distribution of the different head group labeled $\mathrm{Gb}_{3} \mathrm{~s} . \ldots \ldots \ldots \ldots \ldots$

4.4. Head group labeled $\mathrm{Gb}_{3} \mathrm{~s}$ and their influence of the structure properties on liquid ordered phase distribution. . . . . . . . . . . . . . 51

4.5. Liquid ordered phase distribution of the different head group labeled $\mathrm{Gb}_{3} \mathrm{~s}$ compared with different SMs. . . . . . . . . . . 57

5.1. The membrane tension from f-PSM on different functionalized substrates. 73

5.2. The diffusion coefficients from the PSM functionalization measured with iSCAT. . . . . . . . . . . . . . . . . . . . 77

5.3. The diffusion coefficients from the PSM measured with FCS. . . . . . 81

A.1. Comparison of different lipid diffusion constants. . . . . . . . . . . . . 126

A.2. Comparison of different membrane tensions in PSMs. . . . . . . . . . 128

A.3. LMM result of fatty acid labeled $\mathrm{Gb}_{3}$ with no STxB. . . . . . . . . . 130

A.4. LMM result of fatty acid labeled $\mathrm{Gb}_{3}$ with $\mathrm{STxB}$. . . . . . . . . . . . 131

A.5. LMM result of head group labeled $\mathrm{Gb}_{3}$ with $\mathrm{SM}_{\text {porc }}$. . . . . . . . . . . 131

A.6. LMM result of head group labeled $\mathrm{Gb}_{3}$ with $\mathrm{PEG}_{13}$ linker and $\mathrm{SM}_{\text {porc }}$. 132

A.7. LMM result of head group labeled $\mathrm{Gb}_{3}$ with different SMs. . . . . . . 132

A.8. LMM result of head group labeled $\mathrm{Gb}_{3}$ with $\mathrm{PEG}_{13}$ linker and different

SMs. . . . . . . . . . . . . . . . . . 133

A.9. LMM result of membrane tension. . . . . . . . . . . . . . . . 134 


\section{A.4. Lipid diffusion constants}

Table A.1.: Comparison of measured diffusion coefficients for different lipid model systems and lipid phases obtained by different methods. Lipid mixtures are: $\mathrm{A}=\mathrm{SM}_{\text {porc }} / \mathrm{Chol}$, $\mathrm{B}=\mathrm{DOPC} / \mathrm{POPE} / \mathrm{POPS} / \mathrm{Chol}, \mathrm{C}=\mathrm{DOPC} / \mathrm{SM}_{\text {porc }} /$ Chol, $\mathrm{D}=\mathrm{DOPC} / \mathrm{Chol}, \mathrm{E}=\mathrm{DOPC} /$ $\mathrm{SM} \mathrm{C}_{18: 0} / \mathrm{Chol}, \mathrm{F}=\mathrm{DLPC} / \mathrm{DPPC} / \mathrm{Chol}, \mathrm{G}=\mathrm{DLPC} / \mathrm{SM} \mathrm{C}_{18: 0} / \mathrm{Chol}, \mathrm{H}=\mathrm{DMPC} / \mathrm{SM} \mathrm{C}_{18: 0} /$ Chol, I=DOPC/SMegg/Chol, J=DPPC/Chol, K=POPC/Chol, L=DPhPC/DPPC/Chol.

\begin{tabular}{|c|c|c|c|c|c|}
\hline System/Surface & Lipids & Method & Phase & Fluorophore & $D / \mu \mathrm{m}^{2} \mathrm{~s}^{-1}$ \\
\hline $\mathrm{f}-\mathrm{PSM} / \mathrm{SiO}_{x}{ }^{1}$ & DOPC & Z-scan FCS & $l_{\mathrm{d}}$ & ATTO655-DOPE & $11.2 \pm 0.9$ \\
\hline $\mathrm{f}-\mathrm{PSM} / \mathrm{SiO}_{\mathrm{x}}$ & DOPC & Z-scan FCS & $l_{\mathrm{d}}$ & TexasRed-DHPE & $12.4 \pm 1.1$ \\
\hline $\mathrm{f}-\mathrm{PSM} / \mathrm{SiO}_{\mathrm{x}}$ & A $(3: 2)$ & Z-scan FCS & $l_{\mathrm{o}}$ & BODIPY-Chol & $0.78 \pm 0.05$ \\
\hline $\mathrm{f}-\mathrm{PSM} / \mathrm{SiO}_{\mathrm{x}}[$ & $\mathrm{A}(3: 2)$ & Z-scan FCS & $l_{\mathrm{o}}$ & naphthopyrene & $0.57 \pm 0.07$ \\
\hline $\mathrm{f}-\mathrm{PSM} / \mathrm{p}-\mathrm{Si}_{3} \mathrm{~N}_{4}{ }^{2}$ & DOPC & $\mathrm{SPT}$ & $l_{\mathrm{d}}$ & $40 \mathrm{~nm}$ GNP & $2.6 \pm 1.0$ \\
\hline $\mathrm{s}-\mathrm{PSM} / \mathrm{p}-\mathrm{Si}_{3} \mathrm{~N}_{4}$ & DOPC & $\mathrm{SPT}$ & $l_{\mathrm{d}}$ & $40 \mathrm{~nm}$ GNP & $0.8 \pm 0.5$ \\
\hline $\mathrm{f}-\mathrm{PSM} / 6 \mathrm{MH}-\mathrm{Au}^{2}{ }^{2}$ & DOPC & $\mathrm{SPT}$ & $l_{\mathrm{d}}$ & $40 \mathrm{~nm}$ GNP & $1.8 \pm 0.7$ \\
\hline $\mathrm{s}-\mathrm{PSM} / 6 \mathrm{MH}-\mathrm{Au}^{2}$ & DOPC & $\mathrm{SPT}$ & $l_{\mathrm{d}}$ & $40 \mathrm{~nm}$ GNP & $1.9 \pm 0.7$ \\
\hline f-PSM/6MH-Au 267 & B $(5: 2: 1: 2)$ & FCS & $l_{\mathrm{d}}$ & ATTO488-DPPE & $7.7 \pm 0.4$ \\
\hline f-PSM/6MH-Au 267 & B $(5: 2: 1: 2)$ & FCS & $l_{\mathrm{d}}$ & DPPE-KK114 & $7.4 \pm 0.3$ \\
\hline s-PSM/6MH-Au 284 & B $(5: 2: 1: 2)$ & FRAP & $l_{\mathrm{d}}$ & ATTO488-DPPE & $2.8 \pm 0.4$ \\
\hline $\mathrm{f}-\mathrm{PSM} / \mathrm{SiO}_{2} 285$ & DOPC & FRAP & $l_{\mathrm{d}}$ & Rhod-DOPE & 5.2 \\
\hline $\mathrm{s}-\mathrm{PSM} / \mathrm{SiO}_{2} 285$ & DOPC & FRAP & $l_{\mathrm{d}}$ & Rhod-DOPE & $0.26,2.6$ \\
\hline GUV 261 & DOPC & SPT & $l_{\mathrm{d}}$ & $40 \mathrm{~nm}$ GNP & $4.88 \pm 0.04$ \\
\hline GUV 265 & DOPC & Z-scan FCS & $l_{\mathrm{d}}$ & BODIPY 500/510-PC & $7.8 \pm 0.8$ \\
\hline GUV 266 & DOPC & FCS & $l_{\mathrm{d}}$ & ATTO647N-DOPE & $\sim 8.5$ \\
\hline GUV 266 & DOPC & FCS & $l_{\mathrm{d}}$ & BODIPY-Chol & $\sim 8.5$ \\
\hline GUV 170 & DOPC & FCS & $l_{\mathrm{d}}$ & BODIPY-Chol & 7.23 \\
\hline GUV 264 & DOPC & FCS & $l_{\mathrm{d}}$ & DiI- $\mathrm{C}_{18}$ & $6.3 \pm 0.2$ \\
\hline GUV 263 & $\mathrm{C}(2: 2: 1)$ & Z-scan FCS & $l_{\mathrm{d}}$ & DiD-C 18 & $6.1 \pm 0.5$ \\
\hline GUV 263 & $\mathrm{C}(2: 2: 1)$ & Z-scan FCS & $l_{\mathrm{o}}$ & DiD-C 18 & $0.3 \pm 0.1$ \\
\hline GUV 264 & $\mathrm{D}(4: 1)$ & FCS & $l_{\mathrm{d}}$ & DiI- $\mathrm{C}_{18}$ & 4.8 \\
\hline GUV 264 & $\mathrm{D}(1: 2)$ & FCS & $l_{\mathrm{d}}$ & DiI- $\mathrm{C}_{18}$ & 2.4 \\
\hline GUV 264 & $\mathrm{E}(2: 2: 1)$ & FCS & $l_{\mathrm{d}}$ & DiI- $\mathrm{C}_{18}$ & $5.15 \pm 0.15$ \\
\hline GUV 264 & $\mathrm{E}(2: 2: 1)$ & FCS & $l_{\mathrm{o}}$ & DiI- $C_{18}$ & $0.255 \pm 0.058$ \\
\hline GUV 286 & DOPC & FCS & $l_{\mathrm{d}}$ & DiI-C $_{18}$ & $7.0 \pm 1.2$ \\
\hline GUV 286 & $\mathrm{E}(1: 1: 1)$ & FCS & $l_{\mathrm{d}}$ & DiI- $C_{18}$ & $4.8 \pm 0.9$ \\
\hline GUV 286 & $\mathrm{E}(1: 1: 1)$ & FCS & $l_{\mathrm{o}}$ & DiI-C $_{18}$ & $2.0 \pm 1.0$ \\
\hline GUV 287 & $\mathrm{~F}(2: 2: 1)$ & FCS & $l_{\mathrm{d}}$ & DiI- $C_{18}$ & $\sim 4.5$ \\
\hline GUV 287. & $\mathrm{F}(2: 2: 1)$ & FCS & $l_{\mathrm{o}}$ & DiI-C ${ }_{18}$ & $\sim 0.4$ \\
\hline GUV 288 & $\mathrm{G}(1: 1: 2)$ & FCS & $l_{\mathrm{d}}$ & DiI- $C_{18}$ & 0.80 \\
\hline
\end{tabular}

\footnotetext{
${ }^{1}$ Measurements were performed by Thomas Vagedes during his master thesis (table 5.3).

${ }^{2}$ Measurements were done in cooperation with Prof. Dr. Vahid Sandoghdar and were performed by Susann Spindler. The results are published (table 5.2. 147.
} 
A.4. Lipid diffusion constants

\begin{tabular}{|c|c|c|c|c|c|}
\hline System/Surface & Lipids & Method & Phase & Fluorophore & $D / \mu \mathrm{m}^{2} \mathrm{~s}^{-1}$ \\
\hline GUV 288 & G $(1: 1: 2)$ & FCS & $l_{\mathrm{o}}$ & DiI-C $_{18}$ & 0.55 \\
\hline GUV 288 & $\mathrm{H}(1: 1: 2)$ & FCS & $l_{\mathrm{d}}$ & DiI-C $_{18}$ & 0.60 \\
\hline GUV 288 & $\mathrm{H}(1: 1: 2)$ & FCS & $l_{\mathrm{o}}$ & DiI-C $_{18}$ & 0.36 \\
\hline GUV 170 & I $(7: 1: 2)$ & FCS & $l_{\mathrm{o}}$ & BODIPY-Chol & 4.98 \\
\hline GUV 289 & POPC & FCS & $l_{\mathrm{d}}$ & Rhod-PE-POPC & $6.2 \pm 1.1$ \\
\hline GUV 289 & POPC & FRAP & $l_{\mathrm{d}}$ & Rhod-PE-POPC & $3.3 \pm 1.8$ \\
\hline GUV 259 & DOPC & FRAP & $l_{\mathrm{d}}$ & TexasRed-DHPE & $\sim 5$ \\
\hline GPMV 266 & GPMV & FCS & $l_{\mathrm{d}}$ & ATTO647N-DOPE & $\sim 2.5$ \\
\hline GPMV 290 & GPMV & FCS & $l_{\mathrm{d}}$ & Rhod-PE-SOPC & 5.6 \\
\hline GPMV 290 & GPMV & FCS & $l_{\mathrm{o}}$ & Rhod-PE-SOPC & 1.8 \\
\hline SSM/glass 262 & DOPC & SPT & $l_{\mathrm{d}}$ & $10 \mathrm{~nm}$ GNP & $3.01 \pm 0.35$ \\
\hline SSM/glass 262 & DOPC & $\mathrm{SPT}$ & $l_{\mathrm{d}}$ & $15 \mathrm{~nm}$ GNP & $2.87 \pm 0.33$ \\
\hline SSM/glass 262 & DOPC & SPT & $l_{\mathrm{d}}$ & $20 \mathrm{~nm}$ GNP & $2.37 \pm 0.17$ \\
\hline SSM/glass 262 & DOPC & $\mathrm{SPT}$ & $l_{\mathrm{d}}$ & $30 \mathrm{~nm}$ GNP & $2.28 \pm 0.16$ \\
\hline SSM/glass 262 & DOPC & $\mathrm{SPT}$ & $l_{\mathrm{d}}$ & $40 \mathrm{~nm}$ GNP & $2.01 \pm 0.17$ \\
\hline SSM/glass 262 & DOPC & $\mathrm{SPT}$ & $l_{\mathrm{d}}$ & ATTO647N-DOPE & $2.15 \pm 0.67$ \\
\hline $\mathrm{SSM} /$ glass 262 & DOPC & $\mathrm{SPT}$ & $l_{\mathrm{d}}$ & ATTO532-DOPE & $2.63 \pm 0.92$ \\
\hline SSM/glass 260 & DOPC & $\mathrm{SPT}$ & $l_{\mathrm{d}}$ & $40 \mathrm{~nm}$ GNP & 0.22 \\
\hline SSM/glass 291 & $\mathrm{C}(1: 1: 1)$ & SPT & $l_{\mathrm{d}}$ & $40 \mathrm{~nm}$ GNP & 1.1 \\
\hline SSM/glass 291 & $\mathrm{C}(1: 1: 1)$ & $\mathrm{SPT}$ & $l_{\mathrm{o}}$ & $40 \mathrm{~nm}$ GNP & 0.38 \\
\hline GUV/glass 292 & DLPC & FCS & $l_{\mathrm{d}}$ & BODIPY-PC & 3 \\
\hline GUV/glass 292 & $\mathrm{F}(9: 9: 2)$ & FCS & $l_{\mathrm{o}}$ & BODIPY-PC & 0.3 \\
\hline GUV/glass 292 & $\mathrm{~J}(2: 3)$ & FCS & $l_{\mathrm{o}}$ & BODIPY-PC & 0.15 \\
\hline SSM/glass 293 & DOPC & FCS & $l_{\mathrm{d}}$ & BODIPY-Chol & 3.2 \\
\hline SSM/glass 293 & $\mathrm{~K}(7: 3)$ & FCS & $l_{\mathrm{o}}$ & BODIPY-Chol & 1.7 \\
\hline $\mathrm{SSM} / \mathrm{mica} 294$ & $\mathrm{~L}(2: 2: 1)$ & $\mathrm{SPT}$ & $l_{\mathrm{d}}$ & $20 \mathrm{~nm}$ GNP & $1.43 \pm 0.50$ \\
\hline $\mathrm{SSM} / \mathrm{mica} 294$ & $\mathrm{~L}(2: 2: 1)$ & SPT & $l_{\mathrm{o}}$ & $20 \mathrm{~nm}$ GNP & $0.24 \pm 0.12$ \\
\hline $\mathrm{SSM} / \mathrm{mica} 265$ & DOPC & Z-scan FCS & $l_{\mathrm{d}}$ & BODIPY 500/510-PC & $3.1 \pm 0.3$ \\
\hline $\mathrm{SSM} / \mathrm{mica} 295$ & C (10:10:7) & L-scan FCS & $l_{\mathrm{d}}$ & BODIPY-Chol & $6.2 \pm 0.4$ \\
\hline $\mathrm{SSM} /$ mica 295 & C (10:10:7) & L-scan FCS & $l_{\mathrm{o}}$ & BODIPY-Chol & $0.42 \pm 0.02$ \\
\hline $\mathrm{SSM} / \mathrm{mica} 188$ & L $(20: 20: 17)$ & FCS & $l_{\mathrm{d}}$ & DSPE-KK114 & 1.9 \\
\hline $\mathrm{SSM} / \mathrm{mica} 188$ & DOPC & FCS & $l_{\mathrm{d}}$ & DSPE-PEG-KK114 & 9 \\
\hline $\mathrm{SSM} / \mathrm{mica} 188$ & $\mathrm{D}(7: 3)$ & FCS & $l_{\mathrm{d}}$ & DSPE-PEG-KK114 & 2.6 \\
\hline $\mathrm{SSM} /$ mica 188 & L $(20: 20: 17)$ & FCS & $l_{\mathrm{d}}$ & DSPE-PEG-KK114 & 1.8 \\
\hline $\mathrm{SSM} /$ mica 188 & L $(20: 20: 17)$ & FCS & $l_{\mathrm{o}}$ & DSPE-PEG-KK114 & 0.7 \\
\hline $\mathrm{SSM} /$ mica 296 & $\mathrm{E}(3: 3: 2)$ & FCS & $l_{\mathrm{d}}$ & BODIPY-Chol & 6 \\
\hline $\mathrm{SSM} /$ mica 296 & $\mathrm{E}(3: 3: 2)$ & FCS & $l_{\mathrm{o}}$ & BODIPY-Chol & 0.15 \\
\hline
\end{tabular}




\section{A.5. Membrane tension of pore-spanning membranes}

Table A.2.: Comparison measured different membrane tension on different functionalizations of the porous substrates, 2-mercapto-1-ethanol on gold (2ME-Au), 6-mercapto-1hexanol on gold $(6 \mathrm{MH}-\mathrm{Au}), 6 \mathrm{MH}-\mathrm{Au}+\mathrm{O}$-cholesteryl $\mathrm{N}$-(8'-mercapto-3',6'-dioxaoctyl)carbamate (CPEO3), 8-mercapto-1-octanol on gold (8MO-Au), octan-1-thiol on gold (OT-Au), 11-mercapto-1-undecanol on gold (11MUD-Au), 6-mercapto-1-hexanol on gold (6MH-Au), plasma cleaned silicon nitride $\left(\mathrm{p}-\mathrm{Si}_{3} \mathrm{~N}_{4}\right)$ or silicon oxide $\left(\mathrm{SiO}_{\mathrm{x}}\right)$ and different preparation methods, spreading of GUVs or painting the membrane over the porous substrate. The membrane tension $(\sigma)$ was measured also with different lipids and lipid compositions, $\mathrm{A}=\mathrm{POPC} / \mathrm{Chol}, \mathrm{B}=\mathrm{DOPC} / \mathrm{Chol}, \mathrm{C}=\mathrm{DPPC} / \mathrm{Chol}, \mathrm{D}=\mathrm{SM}_{\text {porc }} / \mathrm{Chol}$.

\begin{tabular}{|c|c|c|c|}
\hline Functionalization & Lipids & Preparation & $\sigma / \mathrm{mN} \mathrm{m}^{-1}$ \\
\hline $2 \mathrm{ME}-\mathrm{Au}$ & DOPC & GUV & $1.6 \pm 1.1$ \\
\hline $2 \mathrm{ME}-\mathrm{Au} 239$ & POPC & GUV & $2.4 \pm 0.5$ \\
\hline $2 \mathrm{ME}-\mathrm{Au} 239$ & $\mathrm{~A}(3: 1)$ & GUV & $2.6 \pm 0.9$ \\
\hline $2 \mathrm{ME}-\mathrm{Au} 234$ & POPC & GUV & $2.00 \pm 0.09$ \\
\hline $2 \mathrm{ME}-\mathrm{Au} 234$ & DOPC & GUV & $1.018 \pm 0.014$ \\
\hline 2ME-Au 234 & B $(7: 3)$ & GUV & $3.50 \pm 0.15$ \\
\hline $6 \mathrm{MH}-\mathrm{Au}$ & DOPC & GUV & $0.6 \pm 0.4$ \\
\hline $6 \mathrm{MH}-\mathrm{Au}^{3}$ & DOPC & GUV & $0.4 \pm 0.1$ \\
\hline $6 \mathrm{MH}-\mathrm{Au} 214$ & $\mathrm{DPhPC}$ & GUV & 1.4 \\
\hline $6 \mathrm{MH}-\mathrm{Au}+9.1 \% \mathrm{CPEO} 3214$ & $\mathrm{DPhPC}$ & GUV & 5.0 \\
\hline $\mathrm{OT}-\mathrm{Au} 2235$ & $\mathrm{DPhPC}$ & GUV & $26 \pm 4$ \\
\hline $\mathrm{OT}-\mathrm{Au} 235$ & $\mathrm{DPhPC}$ & painting & $18 \pm 3$ \\
\hline $\mathrm{OT}-\mathrm{Au} 235$ & $\mathrm{DPPC}$ & painting & $31 \pm 3$ \\
\hline $\mathrm{OT}-\mathrm{Au} 235$ & $\mathrm{C}(3: 1)$ & painting & $23 \pm 3$ \\
\hline $8 \mathrm{MO}-\mathrm{Au}$ & DOPC & GUV & $3.2 \pm 0.9$ \\
\hline 11MUD-Au & DOPC & GUV & $2.1 \pm 1.4$ \\
\hline 16MHD-Au & DOPC & GUV & $1.0 \pm 0.4$ \\
\hline $\mathrm{p}-\mathrm{Si}_{3} \mathrm{~N}_{4}$ & DOPC & GUV & $1.6 \pm 0.5$ \\
\hline $\mathrm{p}-\mathrm{Si}_{3} \mathrm{~N}_{4}$ & DOPC & GUV & $1.2 \pm 0.4$ \\
\hline $\mathrm{SiO}_{\mathrm{x}}$ & DOPC & GUV & $10.5 \pm 4.3$ \\
\hline $\mathrm{SiO}_{\mathrm{x}}$ & $\mathrm{D}(3: 2)$ & GUV & $6.7 \pm 4.0$ \\
\hline $\mathrm{SiO}_{2} 235$ & $\mathrm{DPhPC}$ & GUV & $0.15 \pm 0.05$ \\
\hline
\end{tabular}

${ }^{3}$ Measurements were done in cooperation with Prof. Dr. Vahid Sandoghdar. The results are published. 147 


\section{A.6. Fluorescence labeled $\mathbf{G b}_{3}$}

\section{$\mathrm{Gb}_{3}$}

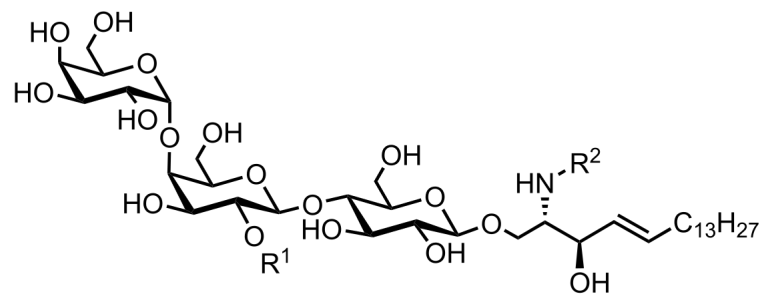

fatty acid labeled $\mathrm{Gb}_{\mathbf{3}}$

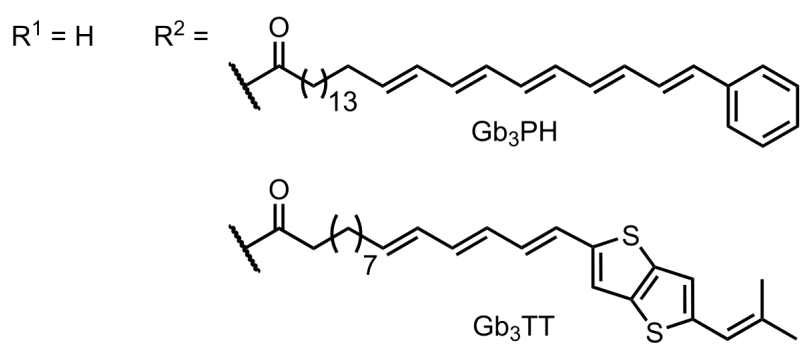

head group labeled $\mathrm{Gb}_{3}$

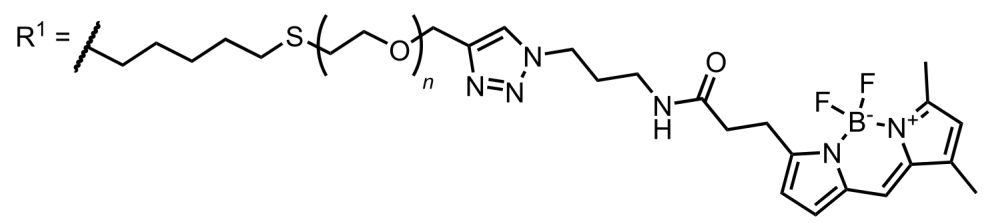

$\mathrm{R}^{2}=\mathrm{C}_{\mathrm{C}_{23} \mathrm{H}_{47}}^{\mathrm{O}}$

$\mathrm{Gb}_{3} \mathrm{PEG}_{13} \mathrm{C}_{24: 0} \mathrm{H}: n=13 \quad \mathrm{~Gb}_{3} \mathrm{PEG}_{13} \mathrm{C}_{24 \cdot 0} \mathrm{OH}: n=13$

$\mathrm{Gb}_{3} \mathrm{PEG}_{3} \mathrm{C}_{24: 0} \mathrm{H}: n=3$<smiles>CCCC(O)C(=O)C(C)C</smiles>

$\mathrm{Gb}_{3} \mathrm{PEG}_{3} \mathrm{C}_{24: 0} \mathrm{OH}: n=3$<smiles>CCC(C)C(C)=O</smiles>

$\mathrm{Gb}_{3} \mathrm{PEG}_{13} \mathrm{C}_{24 \cdot 1} \mathrm{H}: n=13$ $\mathrm{Gb}_{3} \mathrm{PEG}_{3} \mathrm{C}_{24: 1} \mathrm{H}: n=3$

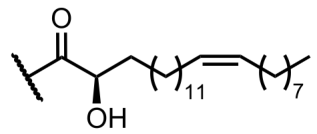

$\mathrm{Gb}_{3} \mathrm{PEG}_{13} \mathrm{C}_{24: 1} \mathrm{OH}: n=13$

$\mathrm{Gb}_{3} \mathrm{PEG}_{3} \mathrm{C}_{24: 1} \mathrm{OH}: n=3$

Scheme A.1.: A schematically overview from all labeled globotriaosyl ceramides $\left(\mathrm{Gb}_{3} \mathrm{~s}\right)$. The ground structure is a $\mathrm{Gb}_{3}$ with two different residues one at the head group $\left(\mathrm{R}^{1}\right)$ and the second the fatty acid $\left(\mathrm{R}^{2}\right)$. The fatty acid labeled $\mathrm{Gb}_{3}$ s have no modification at the head group $\left(\mathrm{R}^{1}=\mathrm{H}\right)$, but there are two different fatty acids, which resulted in the globotriaosyl ceramid phenyl-modified fatty acid $\left(\mathrm{Gb}_{3} \mathrm{PH}\right)$ and globotriaosyl ceramid thienothienylmodified fatty acid $\left(\mathrm{Gb}_{3} \mathrm{TT}\right)$. The head group labeled $\mathrm{Gb}_{3}$ s are modified at the head group with a PEG linker with the linker length $(\mathrm{n}=3,13)$ and a BODIPY. The fatty acid varied also in four different solutions. In total there are eight different structures which were shorten to $\mathrm{Gb}_{3} \mathrm{PEG}_{\mathrm{n}} \mathrm{C}_{24: \Delta} \mathrm{X}$. 


\section{A.7. Statistical analyses}

A linear mixed-effects model (LMM) was fitted to the data in order to evaluate effects of the data.

$$
\text { Data }=X \beta+Z u+\epsilon
$$

The measured data are listed in a long vector. The data belongs to different groups of interest which is set in the fixed effect coefficient matrix $X$. The fixed effects are the influences of interest and stands in the vector $\beta$. The distribution of the data are broadened by systematical distributions (random effects). The coefficient matrix of this systematical errors is $Z$ and the vector of the systematical distribution which has normal distributions as elements is $u$. Additionally a vector of random error $(\epsilon)$ was included which is normal distributed. The equation is optimized by optimizing the normal distributions with a maximum likelihood estimation.

\section{A.7.1. Results of the linear mixed-effects model for the fatty acid labeled globotriaosyl ceramide}

The statistical analysis of the different LMM models are summarized in the following short tables. The differences are calculated as following $\Delta$ FattyAcid $=\% l_{\mathrm{o}}\left(\mathrm{Gb}_{3} \mathrm{PH}\right)$ $-\% l_{\mathrm{o}}\left(\mathrm{Gb}_{3} \mathrm{TT}\right), \Delta \mathrm{STxB}=\% l_{\mathrm{o}}(+\mathrm{STxB})-\% l_{\mathrm{o}}(-\mathrm{STxB})$. The LMM has a starting population, which were influenced by fixed-effects and random-effects. The effects with fixed influences, like the influence of the fatty acid to the $l_{\mathrm{o}}$ phase distribution $\left(\% l_{\mathrm{o}}\right)$ of the head group labeled $\mathrm{Gb}_{3}$, were shorten in the LMM equation as an addend. Effects, which influences the distribution of the statistic are random-effects, like the preparation days or the different GUVs. This random effects were written in the LMM equation like $(1 \mid<$ random effect $>)$.

Table A.3.: The used model: liquid ordered phase distribution $\left(\% l_{\mathrm{o}}\right)=\mathrm{Gb}_{3} \mathrm{PH}+\Delta$ FattyAcid $+(1 \mid$ Day $)+(1 \mid \mathrm{GUV})$. All two different fatty acid labeled $\mathrm{Gb}_{3}$ were used in GUV composed of $\mathrm{DOPC} / \mathrm{SM}_{\text {porc }} / \mathrm{Chol} / \mathrm{Gb}_{3} / \mathrm{Dy} 731-\mathrm{DOPE}$ (39:35:20:5:1) before STxB incubation; $\mathrm{R}^{2}=0.6710$

\begin{tabular}{lrr} 
Difference & $\Delta \% l_{\mathrm{o}}$ & p-Value \\
\hline $\mathrm{Gb}_{3} \mathrm{PH}$ & $0.40 \pm 0.02$ & $2.4 \times 10^{-205}$ \\
$\Delta$ FattyAcid & $0.16 \pm 0.02$ & $3.1 \times 10^{-22}$
\end{tabular}


Table A.4.: The used model: liquid ordered phase distribution $\left(\% l_{\mathrm{o}}\right)=\mathrm{Gb}_{3} \mathrm{PH}+\Delta$ FattyAcid $+\Delta \mathrm{STxB}(1 \mid$ Day $)+(1 \mid$ GUV $)$. All two different fatty acid labeled $\mathrm{Gb}_{3}$ were used in GUV composed of $\mathrm{DOPC} / \mathrm{SM}_{\text {porc }} / \mathrm{Chol} / \mathrm{Gb}_{3} / \mathrm{Dy} 731-\mathrm{DOPE}$ (39:35:20:5:1) before and after STxB incubation; $\mathrm{R}^{2}=0.6266$

\begin{tabular}{lrr} 
Difference & $\Delta \% l_{\mathrm{o}}$ & $\mathrm{p}$-Value \\
\hline $\mathrm{Gb}_{3} \mathrm{PH}$ & $0.39 \pm 0.02$ & $3.9 \times 10^{-286}$ \\
$\Delta$ FattyAcid & $0.16 \pm 0.02$ & $1.7 \times 10^{-44}$ \\
$\Delta \mathrm{STxB}$ & $-0.01 \pm 0.02$ & 0.19
\end{tabular}

\section{A.7.2. Results of the linear mixed-effects model for the head group labeled globotriaosyl ceramide}

The statistical analysis of the different LMM models are summarized in the following short tables. The differences are calculated as following $\triangle \mathrm{PEG}=\% l_{\mathrm{o}}\left(\mathrm{PEG}_{13}\right)$ $\% l_{\mathrm{o}}\left(\mathrm{PEG}_{3}\right), \Delta \mathrm{C}_{24}=\% l_{\mathrm{o}}\left(\mathrm{C}_{24: 0}\right)-\% l_{\mathrm{o}}\left(\mathrm{C}_{24: 1}\right)$ and $\Delta \mathrm{OH}=\% l_{\mathrm{o}}(\mathrm{H})-\% l_{\mathrm{o}}(\mathrm{OH})$. The LMM has a starting population, which were influenced by fixed-effects and random-effects. The effects with fixed influences, like the influence of the linker length to the $\% l_{\text {o }}$ of the head group labeled $\mathrm{Gb}_{3}$, were shorten in the LMM equation as an addend. Effects, which influences the distribution of the statistic are random-effects, like the preparation days or the different GUVs. This random effects were written in the LMM equation like $(1 \mid<$ random effect $>)$.

Measurements with $S M_{\text {porc }}$

Table A.5.: The used model: liquid ordered phase distribution $\left(\% l_{\mathrm{o}}\right)=\mathrm{Gb}_{3} \mathrm{PEG}_{13} \mathrm{C}_{24: 0} \mathrm{H}+\Delta \mathrm{PEG}+\Delta \mathrm{C}_{24}+\Delta \mathrm{OH}+(1 \mid$ Day $)+(1 \mid$ GUV $)$. All eight different head group labeled $\mathrm{Gb}_{3}$ were used in GUV with the lipid mixture DOPC/ $\mathrm{SM}_{\text {porc }} / \mathrm{Chol} / \mathrm{Gb}_{3} /$ Dy731-DOPE (39:39:20:1:1); $\mathrm{R}^{2}=0.9201$

\begin{tabular}{lrr} 
Difference & $\Delta \% l_{\mathrm{o}}$ & $\mathrm{p}$-Value \\
\hline $\mathrm{Gb}_{3} \mathrm{PEG}_{13} \mathrm{C}_{24: 0} \mathrm{H}$ & $0.72 \pm 0.03$ & $1.4 \times 10^{-250}$ \\
$\Delta \mathrm{PEG}$ & $0.27 \pm 0.03$ & $1.3 \times 10^{-32}$ \\
$\Delta \mathrm{C}_{24}$ & $0.19 \pm 0.03$ & $1.6 \times 10^{-16}$ \\
$\Delta \mathrm{OH}$ & $0.01 \pm 0.03$ & 0.67
\end{tabular}


Table A.6.: The used model: liquid ordered phase distribution $\left(\% l_{\mathrm{o}}\right)=\mathrm{Gb}_{3} \mathrm{PEG}_{13} \mathrm{C}_{24: 0} \mathrm{H}$ $+\Delta \mathrm{C}_{24}+\Delta \mathrm{OH}+(1 \mid$ Day $)+(1 \mid \mathrm{GUV})$. The head group labeled $\mathrm{Gb}_{3}$ with the long PEG linker were used in GUV with the lipid mixture DOPC $/ \mathrm{SM}_{\text {porc }} / \mathrm{Chol} / \mathrm{Gb}_{3} / \mathrm{Dy} 731-\mathrm{DOPE}$ $(39: 39: 20: 1: 1) ; R^{2}=0.8695$

\begin{tabular}{lrr} 
Difference & $\Delta \% l_{\mathrm{o}}$ & $\mathrm{p}$-Value \\
\hline $\mathrm{Gb}_{3} \mathrm{PEG}_{13} \mathrm{C}_{24: 0} \mathrm{H}$ & $0.74 \pm 0.03$ & $9.4 \times 10^{-186}$ \\
$\Delta \mathrm{C}_{24}$ & $0.24 \pm 0.04$ & $3.7 \times 10^{-15}$ \\
$\Delta \mathrm{OH}$ & $0.01 \pm 0.04$ & 0.76
\end{tabular}

\section{Measurements with different sphingomyelin species}

Table A.7.: The used model: liquid ordered phase distribution $\left(\% l_{\mathrm{o}}\right)=\mathrm{Gb}_{3} \mathrm{PEG}_{13} \mathrm{C}_{24: 0} \mathrm{H}$ in $\mathrm{SMC}_{16: 0}+\Delta \mathrm{SM}+\Delta \mathrm{PEG}+\Delta \mathrm{C}_{24}+\Delta \mathrm{OH}+(1 \mid$ Day $)+(1 \mid \mathrm{GUV})$. All eight different head group labeled $\mathrm{Gb}_{3}$ were used in GUV with the lipid mixture DOPC/SM/Chol/ $/ \mathrm{Gb}_{3} /$ Dy731-DOPE (39:39:20:1:1); $\mathrm{R}^{2}=0.9495$

\begin{tabular}{lrr} 
Difference & $\Delta \% l_{\mathrm{o}}$ & $\mathrm{p}$-Value \\
\hline $\mathrm{Gb}_{3} \mathrm{PEG}_{13} \mathrm{C}_{24: 0} \mathrm{H}$ in $\mathrm{SM} \mathrm{C}_{16: 0}$ & $0.48 \pm 0.03$ & $1.2 \times 10^{-75}$ \\
$\Delta \mathrm{SM} \mathrm{C}_{16: 0}-\mathrm{SM} \mathrm{C}_{18: 0}$ & $-0.03 \pm 0.03$ & 0.36 \\
$\Delta \mathrm{SM} \mathrm{C}_{16: 0}-\mathrm{SM} \mathrm{C}_{20: 0}$ & $-0.21 \pm 0.03$ & $1.8 \times 10^{-12}$ \\
$\Delta \mathrm{SM} \mathrm{C}_{16: 0}-\mathrm{SM} \mathrm{C}_{22: 0}$ & $-0.19 \pm 0.03$ & $4.7 \times 10^{-11}$ \\
$\Delta \mathrm{SM} \mathrm{C}_{16: 0}-\mathrm{SM} \mathrm{C}_{24: 0}$ & $-0.19 \pm 0.03$ & $8.1 \times 10^{-12}$ \\
$\Delta \mathrm{SM} \mathrm{C}_{18: 0}-\mathrm{SM} \mathrm{C}_{20: 0}$ & $-0.18 \pm 0.03$ & $7.4 \times 10^{-10}$ \\
$\Delta \mathrm{SM} \mathrm{C}_{18: 0}-\mathrm{SM} \mathrm{C}_{22: 0}$ & $-0.17 \pm 0.03$ & $1.2 \times 10^{-8}$ \\
$\Delta \mathrm{SM} \mathrm{C}_{18: 0}-\mathrm{SM} \mathrm{C}_{24: 0}$ & $-0.17 \pm 0.03$ & $2.9 \times 10^{-9}$ \\
$\Delta \mathrm{SM} \mathrm{C}_{20: 0}-\mathrm{SM} \mathrm{C}_{22: 0}$ & $0.01 \pm 0.04$ & 0.65 \\
$\Delta \mathrm{SM} \mathrm{C}_{20: 0}-\mathrm{SM} \mathrm{C}_{24: 0}$ & $0.01 \pm 0.03$ & 0.78 \\
$\Delta \mathrm{SM} \mathrm{C}_{22: 0}-\mathrm{SM} \mathrm{C}_{24: 0}$ & $-0.01 \pm 0.03$ & 0.86 \\
$\Delta \mathrm{PEG}$ & $0.31 \pm 0.02$ & $2.6 \times 10^{-65}$ \\
$\Delta \mathrm{C}_{24}$ & $0.16 \pm 0.02$ & $6.3 \times 10^{-18}$ \\
$\Delta \mathrm{OH}$ & $-0.03 \pm 0.02$ & 0.15
\end{tabular}


Table A.8.: The used model: liquid ordered phase distribution $\left(\% l_{\mathrm{o}}\right)=\mathrm{Gb}_{3} \mathrm{PEG}_{13} \mathrm{C}_{24: 0} \mathrm{H}$ in $\mathrm{SM} \mathrm{C}_{16: 0}+\Delta \mathrm{SM}+\Delta \mathrm{C}_{24}+\Delta \mathrm{OH}+(1 \mid$ Day $)+(1 \mid \mathrm{GUV})$. The head group labeled $\mathrm{Gb}_{3}$ with the long PEG linker were used in GUV with the lipid mixture DOPC/SM/Chol/ $\mathrm{Gb}_{3} /$ Dy731-DOPE (39:39:20:1:1); $\mathrm{R}^{2}=0.9178$

\begin{tabular}{ccc} 
Difference & $\Delta \% l_{\mathrm{o}}$ & $\mathrm{p}$-Value \\
\hline $\mathrm{Gb}_{3} \mathrm{PEG}_{13} \mathrm{C}_{24: 0} \mathrm{H}_{\text {in }} \mathrm{SM} \mathrm{C}_{16: 0}$ & $0.46 \pm 0.03$ & $3.3 \times 10^{-53}$ \\
$\Delta \mathrm{SM} \mathrm{C}_{16: 0^{-}} \mathrm{SM} \mathrm{C}_{18: 0}$ & $-0.09 \pm 0.04$ & $8.8 \times 10^{-3}$ \\
$\Delta \mathrm{SM} \mathrm{C}_{16: 0^{-}} \mathrm{SM} \mathrm{C}_{20: 0}$ & $-0.25 \pm 0.04$ & $1.1 \times 10^{-11}$ \\
$\Delta \mathrm{SM} \mathrm{C}_{16: 0^{-}} \mathrm{SM} \mathrm{C}_{22: 0}$ & $-0.25 \pm 0.04$ & $4.7 \times 10^{-12}$ \\
$\Delta \mathrm{SM} \mathrm{C}_{16: 0^{-}} \mathrm{SM} \mathrm{C}_{24: 0}$ & $-0.29 \pm 0.04$ & $2.5 \times 10^{-15}$ \\
$\Delta \mathrm{SM} \mathrm{C}_{18: 0^{-}} \mathrm{SM} \mathrm{C}_{20: 0}$ & $-0.16 \pm 0.04$ & $1.0 \times 10^{-5}$ \\
$\Delta \mathrm{SM} \mathrm{C}_{18: 0^{-}} \mathrm{SM} \mathrm{C}_{22: 0}$ & $-0.16 \pm 0.04$ & $5.4 \times 10^{-6}$ \\
$\Delta \mathrm{SM} \mathrm{C}_{18: 0^{-}} \mathrm{SM} \mathrm{C}_{24: 0}$ & $-0.20 \pm 0.04$ & $2.6 \times 10^{-8}$ \\
$\Delta \mathrm{SM} \mathrm{C}_{20: 0^{-}} \mathrm{SM} \mathrm{C}_{22: 0}$ & $-0.00 \pm 0.04$ & 0.90 \\
$\Delta \mathrm{SM} \mathrm{C}_{20: 0^{-}} \mathrm{SM} \mathrm{C}_{24: 0}$ & $-0.04 \pm 0.04$ & 0.27 \\
$\Delta \mathrm{SM} \mathrm{C}_{22: 0^{-}} \mathrm{SM} \mathrm{C}_{24: 0}$ & $-0.04 \pm 0.04$ & 0.33 \\
$\Delta \mathrm{C}_{24}$ & $0.22 \pm 0.03$ & $6.4 \times 10^{-21}$ \\
$\Delta \mathrm{OH}$ & $0.02 \pm 0.03$ & 0.52
\end{tabular}

\section{A.7.3. Statistical analyzing of Membrane tension by using the LMM}

The statistical analysis of the LMM model is summarized in the following short tables. The differences of the membrane tension according there functionalization is exlained in the tables. The LMM has a starting population, which were influenced by fixed-effects and random-effects. The effects with fixed influences were shorten in the LMM equation as an addend. Effects, which influences the distribution of the statistic are random-effects, like the preparation days. This random effects were written in the LMM equation like $(1 \mid<$ random effect $>)$. 
Table A.9.: The used model was, membrane tension $(\sigma)=2 \mathrm{ME}-\mathrm{Au}+\Delta$ functionalization $+(1 \mid$ Day $) . \mathrm{R}^{2}=0.8628$. The membrane tension $(\sigma)$ of the f-PSM composed of DOPC/ ATTO488-DPPE (99.5:0.5) was measured on different functionalizations. The lipid composition of the $l_{\mathrm{o}}$ phase on $\mathrm{SiO}_{\mathrm{x}}$ functionalized substrate was $\mathrm{SM}_{\text {porc }} / \mathrm{Chol} / \mathrm{ATTO} 655-\mathrm{DOPE}$ (59.5:39.5:1). The analyze method is described in chapter 3.3 .3 .

\begin{tabular}{|c|c|c|}
\hline Difference & $\Delta \sigma \mathrm{mN} \mathrm{m}^{-1}$ & p-Value \\
\hline $2 \mathrm{ME}-\mathrm{Au}$ & $2.1 \pm 1.1$ & 0.047 \\
\hline$\triangle 2 \mathrm{ME}-\mathrm{Au}-6 \mathrm{MH}-\mathrm{Au}$ & $1.3 \pm 2.0$ & 0.50 \\
\hline$\triangle 2 \mathrm{ME}-\mathrm{Au}-8 \mathrm{MO}-\mathrm{Au}$ & $-1.3 \pm 1.7$ & 0.52 \\
\hline$\Delta 2 \mathrm{ME}-\mathrm{Au}-11 \mathrm{MUD}-\mathrm{Au}$ & $0.2 \pm 1.9$ & 0.42 \\
\hline$\Delta 2 \mathrm{ME}-\mathrm{Au}-16 \mathrm{MHD}-\mathrm{Au}$ & $1.0 \pm 1.7$ & 0.92 \\
\hline$\Delta 2 \mathrm{ME}-\mathrm{Au}-\mathrm{p}-\mathrm{Si}_{3} \mathrm{~N}_{4}$ & $-0.5 \pm 2.5$ & 0.84 \\
\hline$\Delta 2 \mathrm{ME}-\mathrm{Au}-\mathrm{SiO}_{\mathrm{x}}$ & $-7.0 \pm 1.5$ & $1.4 \times 10^{-6}$ \\
\hline$\Delta 2 \mathrm{ME}-\mathrm{Au}-\mathrm{SiO}_{\mathrm{x}} l_{\mathrm{o}}$ & $5.1 \pm 1.5$ & $4.5 \times 10^{-4}$ \\
\hline $6 \mathrm{MH}-\mathrm{Au}$ & $0.73 \pm 1.70$ & 0.66 \\
\hline$\Delta 6 \mathrm{MH}-\mathrm{Au}-8 \mathrm{MO}-\mathrm{Au}$ & $-2.7 \pm 2.2$ & 0.21 \\
\hline$\Delta 6 \mathrm{MH}-\mathrm{Au}-11 \mathrm{MUD}-\mathrm{Au}$ & $-1.1 \pm 2.3$ & 0.62 \\
\hline$\Delta 6 \mathrm{MH}-\mathrm{Au}-16 \mathrm{MHD}-\mathrm{Au}$ & $-0.3 \pm 2.1$ & 0.89 \\
\hline$\Delta 6 \mathrm{MH}-\mathrm{Au}-\mathrm{p}-\mathrm{Si}_{3} \mathrm{~N}_{4}$ & $0.8 \pm 2.8$ & 0.77 \\
\hline$\Delta 6 \mathrm{MH}-\mathrm{Au}-\mathrm{SiO}_{\mathrm{x}}$ & $-8.3 \pm 2.0$ & $2.0 \times 10^{-5}$ \\
\hline$\Delta 6 \mathrm{MH}-\mathrm{Au}-\mathrm{SiO}_{\mathrm{x}} l_{\mathrm{o}}$ & $-6.4 \pm 2.0$ & $1.0 \times 10^{-3}$ \\
\hline $8 \mathrm{MO}-\mathrm{Au}$ & $3.4 \pm 1.4$ & $9.6 \times 10^{-4}$ \\
\hline$\Delta 8 \mathrm{MO}-\mathrm{Au}-11 \mathrm{MUD}-\mathrm{Au}$ & $1.5 \pm 2.1$ & 0.46 \\
\hline$\Delta 8 \mathrm{MO}-\mathrm{Au}-16 \mathrm{MHD}-\mathrm{Au}$ & $2.4 \pm 1.9$ & 0.20 \\
\hline$\Delta 8 \mathrm{MO}-\mathrm{Au}-\mathrm{p}-\mathrm{Si}_{3} \mathrm{~N}_{4}$ & $1.8 \pm 2.6$ & 0.48 \\
\hline$\Delta 8 \mathrm{MO}-\mathrm{Au}-\mathrm{SiO}_{\mathrm{x}}$ & $-5.7 \pm 1.7$ & $5.9 \times 10^{-4}$ \\
\hline$\Delta 8 \mathrm{MO}-\mathrm{Au}-\mathrm{SiO}_{\mathrm{x}} l_{\mathrm{o}}$ & $-3.8 \pm 1.7$ & 0.023 \\
\hline$\Delta 6 \mathrm{MH}-\mathrm{Au}-\mathrm{SiO}_{\mathrm{x}} l_{\mathrm{o}}$ & $-6.4 \pm 2.0$ & $1.0 \times 10^{-3}$ \\
\hline 11MUD-Au & $1.9 \pm 1.6$ & 0.24 \\
\hline$\Delta 11 \mathrm{MUD}-\mathrm{Au}-16 \mathrm{MHD}-\mathrm{Au}$ & $0.86 \pm 2.10$ & 0.67 \\
\hline$\Delta 11 \mathrm{MUD}-\mathrm{Au}-\mathrm{p}-\mathrm{Si}_{3} \mathrm{~N}_{4}$ & $0.32 \pm 2.80$ & 0.91 \\
\hline$\Delta 11 \mathrm{MUD}-\mathrm{Au}-\mathrm{SiO}_{\mathrm{x}}$ & $-7.2 \pm 1.9$ & $1.3 \times 10^{-4}$ \\
\hline$\Delta 11 \mathrm{MUD}-\mathrm{Au}-\mathrm{SiO}_{\mathrm{x}} l_{\mathrm{o}}$ & $-5.3 \pm 1.9$ & $5.0 \times 10^{-3}$ \\
\hline 16MHD-Au & $1.0 \pm 1.3$ & 0.43 \\
\hline$\Delta 16 \mathrm{MHD}-\mathrm{Au}-\mathrm{p}-\mathrm{Si}_{3} \mathrm{~N}_{4}$ & $0.54 \pm 2.60$ & 0.83 \\
\hline$\Delta 16 \mathrm{MHD}-\mathrm{Au}-\mathrm{SiO}_{\mathrm{x}}$ & $-8.1 \pm 1.7$ & $9.4 \times 10^{-7}$ \\
\hline
\end{tabular}




\begin{tabular}{lrr} 
Difference & $\Delta \sigma \mathrm{mN} \mathrm{m}^{-1}$ & $\mathrm{p}$-Value \\
\hline$\Delta 16 \mathrm{MHD}-\mathrm{Au}-\mathrm{SiO}_{\mathrm{x}} l_{\mathrm{o}}$ & $-6.1 \pm 1.7$ & $1.9 \times 10^{-4}$ \\
$\mathrm{p}-\mathrm{Si}_{3} \mathrm{~N}_{4}$ & $1.6 \pm 2.2$ & 0.48 \\
$\Delta \mathrm{p}-\mathrm{Si}_{3} \mathrm{~N}_{4}-\mathrm{SiO}_{\mathrm{x}}$ & $-7.5 \pm 2.5$ & $2.1 \times 10^{-3}$ \\
$\Delta \mathrm{p}-\mathrm{Si}_{3} \mathrm{~N}_{4}-\mathrm{SiO}_{\mathrm{x}} l_{\mathrm{o}}$ & $-5.6 \pm 2.5$ & 0.022 \\
$\mathrm{SiO}_{\mathrm{x}}$ & $9.07 \pm 1.01$ & $1.6 \times 10^{-18}$ \\
$\Delta \mathrm{SiO}_{\mathrm{x}}-\mathrm{SiO}_{\mathrm{x}} l_{\mathrm{o}}$ & $1.9 \pm 1.5$ & 0.18 \\
$\mathrm{SiO}_{\mathrm{x}} l_{\mathrm{o}}$ & $7.2 \pm 1.0$ & $3.9 \times 10^{-12}$
\end{tabular}

\section{A.8. Chemicals and consumables}

2-mercapto-1-ethanol

3-aminopropyltrimethoxysilane

6-mercapto-1-hexanol

8-mercapto-1-octanol

octan-1-thiol

12-mercapto-1-dodecanol

16-mercapto-1-hexadecanol

Ar

$\mathrm{Au}, 99.99 \%$

ATTO488-DOPE

ATTO488-DPPE

ATTO532-DOPE

ATTO655-DOPE

cb-DOPE

BL-AC40TS-C2

BODIPY-Chol

BSA

$\mathrm{CaCl}_{2} \cdot\left(\mathrm{H}_{2} \mathrm{O}\right)_{2}$

Chloroform

Chol

Cu-band
Sigma-Aldrich, Taufkirchen, Germany

Sigma-Aldrich, Taufkirchen, Germany

Sigma-Aldrich, Taufkirchen, Germany

Sigma-Aldrich, Taufkirchen, Germany

Sigma-Aldrich, Taufkirchen, Germany

Dojindo EU GmbH, Munich, Germany

Santa Cruz Biotechnology, Inc., Heidelberg,

Germany

Air Liquide Deutschland GmbH, Düsseldorf,

Germany

Allgemeine Gold- und Silberscheideanstalt,

Pforzheim, Germany

ATTO-TEC, Siegen, Germany

ATTO-TEC, Siegen, Germany

ATTO-TEC, Siegen, Germany

ATTO-TEC, Siegen, Germany

Avanti Polar Lipids, Alabaster, AL, US

Olympus, Tokio, Japan

Avanti Polar Lipids, Alabaster, AL, US

Carl Roth GmbH, Karlsruhe, Germany

Merck KGaA, Darmstadt, Germany

VWR International, Darmstadt, Germany

Sigma-Aldrich, Taufkirchen, Germany

Präzisions Glas \& Optik GmbH, Iserlohn, Germany 
Cy3 STxB

DOPC

Dy731-DOPE

Ethanol

$\mathrm{Gb}_{3} \mathrm{PEG}_{3} \mathrm{C}_{24: 0} \mathrm{H}$

$\mathrm{Gb}_{3} \mathrm{PEG}_{3} \mathrm{C}_{24: 1} \mathrm{H}$

$\mathrm{Gb}_{3} \mathrm{PEG}_{3} \mathrm{C}_{24: 0} \mathrm{OH}$

$\mathrm{Gb}_{3} \mathrm{PEG}_{3} \mathrm{C}_{24: 1} \mathrm{OH}$

$\mathrm{Gb}_{3} \mathrm{PEG}_{13} \mathrm{C}_{24: 0} \mathrm{H}$

$\mathrm{Gb}_{3} \mathrm{PEG}_{13} \mathrm{C}_{24: 1} \mathrm{H}$

$\mathrm{Gb}_{3} \mathrm{PEG}_{13} \mathrm{C}_{24: 0} \mathrm{OH}$

$\mathrm{Gb}_{3} \mathrm{PEG}_{13} \mathrm{C}_{24: 1} \mathrm{OH}$

$\mathrm{Gb}_{3} \mathrm{PH}$

$\mathrm{Gb}_{3 \text { porc }}$

$\mathrm{Gb}_{3} \mathrm{TT}$

GNP

iso-propanol

ITO-slides

$\mathrm{KCl}$

$\mathrm{KH}_{2} \mathrm{PO}_{4}$

Methoxy PEG succinimidy carbonate $(5 \mathrm{kDa})$

$\mathrm{N}_{2}$

$n$-propanol

Methanol

$\mathrm{NaCl}$

$\mathrm{Na}_{2} \mathrm{HPO}_{4}$

naphthopyrene

$\mathrm{O}_{2}$

PageRuler ${ }^{\mathrm{TM}}$

Polycarbonate membranes

Porous $\mathrm{Si}_{3} \mathrm{~N}_{4}$ substrates

$\mathrm{SiO}$
Provided by Prof. Dr. Winfried Römer

Avanti Polar Lipids, Alabaster, AL, US

Provided by Prof. Dr. Daniel B. Werz

Carl Roth GmbH, Karlsruhe, Germany

Provided by Prof. Dr. Daniel B. Werz

Provided by Prof. Dr. Daniel B. Werz

Provided by Prof. Dr. Daniel B. Werz

Provided by Prof. Dr. Daniel B. Werz

Provided by Prof. Dr. Daniel B. Werz

Provided by Prof. Dr. Daniel B. Werz

Provided by Prof. Dr. Daniel B. Werz

Provided by Prof. Dr. Daniel B. Werz

Provided by Prof. Dr. Daniel B. Werz

Matreya, State College, PA, US

Provided by Prof. Dr. Daniel B. Werz

BBI, Cardiff, UK

Carl Roth GmbH, Karlsruhe, Germany

Präzisions Glas \& Optik GmbH, Iserlohn, Germany

Sigma-Aldrich, Taufkirchen, Germany

Merck KGaA, Darmstadt, Germany

Nanocs Inc., Boston, MA, US

Air Liquide Deutschland GmbH, Düsseldorf, Germany

Carl Roth GmbH, Karlsruhe, Germany

Carl Roth GmbH, Karlsruhe, Germany

Sigma-Aldrich, Taufkirchen, Germany

Merck KGaA, Darmstadt, Germany

TCI Deutschland GmbH, Eschborn, Germany

Air Liquide Deutschland GmbH, Düsseldorf,

Germany

Fisher Scientific GmbH, Schwerte, Germany

Avestin, Ottawa, Canada

Aquamarijn Micro Filtration BV, Zutphen,

Netherlands

Merck KGaA, Darmstadt, Germany 
$\mathrm{SM} \mathrm{C}_{16: 0}$

$\mathrm{SM} \mathrm{C}_{18: 0}$

$\mathrm{SM} \mathrm{C}_{20: 0}$

$\mathrm{SM} \mathrm{C}_{22: 0}$

$\mathrm{SM} \mathrm{C}_{24: 0}$

$\mathrm{SM}_{\text {porc }}$

SPR sensor chips (bare gold)

STxB

STxB

Sucrose

TexasRed-DHPE

$\mathrm{Ti}$

Tris

Ultra low range marker
Avanti Polar Lipids, Alabaster, AL, US

Avanti Polar Lipids, Alabaster, AL, US

Matreya, State College, PA, US

Matreya, State College, PA, US

Avanti Polar Lipids, Alabaster, AL, US

Avanti Polar Lipids, Alabaster, AL, US

XanTec bioanalytics GmbH, Düsseldorf, Germany

Provided by Prof. Dr. Daniel Huster

Provided by Prof. Dr. Winfried Römer

Carl Roth GmbH, Karlsruhe, Germany

Sigma-Aldrich, Taufkirchen, Germany

Elektronen Optik Service GmbH, Dortmund,

Germany

Carl Roth GmbH, Karlsruhe, Germany

Sigma-Aldrich, Taufkirchen, Germany

\section{A.9. Software}

Fiji

Matlab 2014b

Matlab 2017b

Origin Pro8.5G
Schindelin et al. 297

Mathworks, Natick, MA, US

Mathworks, Natick, MA, US

OriginLab Corporation, Northhampton, US

\section{A.10. Equipment}

BX 51

BX 53

Coating system MED020

Frequency generator Agilent 33220A

FluoView 1200

Heating chamber

LEO supra-35 microscope

LSM 710

LSM 880
Olympus, Tokio, Japan

Olympus, Tokio, Japan

Bal-TEc, Leica, Wetzlar, Germany

Agilent Technology, Santa Clara, CA, US

Olympus, Tokio, Japan

Binder, Tuttlingen, Germany

Carl Zeiss, Jena, Germany

Carl Zeiss, Jena, Germany

Carl Zeiss, Jena, Germany 
MilliQ Gradient A10

MFP-3D classic

Nanowizard 4

pH-meter Calimatic 766

Sputtercoater

Plasma cleaner PDC 32 G-2

Tip sonifier Sonoplus HD2070

Ultrasonic bath Sonorex RK $255 \mathrm{H}$

Vacuum drying chamber
Merck Millipore, Darmstadt, Germany

Asylum Research, Santa Barbara, CA, US

JPK Instruments, Berlin, Germany

Knick, Berlin, Germany

Cressington Scientific Instruments, Watford, UK

Harrick Plasma, Ithaca, NY, US

Bandelin, Berlin, Germany

Bandelin, Berlin, Germany

Binder, Tuttlingen, Germany 


\section{Curriculum vitae}

\section{Personal data}

Name: Jeremias Sibold

Date of birth: 18.04 .1990

Place of birth: Donaueschingen, Germany

Citizenship: German

\section{Carreer}

2015-2019 Ph.D. thesis at the Georg-August University, Göttingen (Prof. Dr. C. Steinem), title of the thesis: Phase separation of biomimetic membranes: Influence of the substrate adhesion or the glycosphingolipid structure

2013-2015 graduate studies in chemistry at the Georg-August University, Göttingen, degree: Master of Science, grade: 1.8, title of the master thesis: Mechanical properties of pore-spanning membranes: Influence of osmolarity gradients, grade: 1.3

2010-2013 undergraduate studies in chemistry at the Georg-August University, Göttingen, degree: Bachelor of Science, grade: 1.9, title of the bachelor thesis: Theoretical study of $\beta$-Peptides, grade: 1.3 2009-2010 civilian service at Hofgemeinschaft für Heilende Arbeit e.V., care of disabled people in Khena, Germany

2006-2009 Allgemeine Hochschulreife at Merian Schule Biotechnologisches Gymnasium, Freiburg, degree: Abitur, grade: 1.5

\section{Awards}

Best Poster Award 2018 1st at the Biennial Meeting of the German Biophysical Society 2018, Düsseldorf, Germany Travel grant to the 19th IUPAB and 11th EBSA congress in Edinburgh, UK, from the Deutschen Gesellschaft für Biophysik 


\section{Scientific contributions}

\section{Manuscript}

2019

Sibold J., Tewaag V., Vagedes T., Mey I. \& Steinem C. Phase separation induced by surface adhesion (2019). in preparation.

\section{Peer-reviewed publications}

Sibold J., Kettelhoit K., Vuong L., Liu F., Werz D. B. \& Steinem C. Synthesis of Head Group Labeled Gb3 Glycosphingolipids and Their Distribution in Phase-Separated Giant Unilamellar Vesicles. Angew. Chem. Int. Ed. 58, 17805-17813 (2019).

Bosse M., Sibold J., Scheidt H. A., Patalag L. J., Kettelhoit K., Ries A., Werz D. B., Steinem C. \& Huster D. Shiga Toxin Binding Alters Lipid Packing and Domain Structure of $\mathrm{Gb}_{3}$-Containing Membranes: A Solid-State NMR Study. Phys. Chem. Chem. Phys. 21, 15630-15638 (2019).

2018 Spindler S., Sibold J., Gholami Mahmoodabadi R., Steinem C. \& Sandoghdar V. High-Speed Microscopy of Diffusion in PoreSpanning Lipid Membranes. Nano Lett. 18, 5262-5271 (2018).

Teske N., Sibold J., Schumacher J., Teiwes N. K., Gleisner M., Mey I. \& Steinem C. Continuous Pore-Spanning Lipid Bilayers On Silicon Oxide-Coated Porous Substrates. Langmuir 33, 1417514183 (2017).

2017 Patalag L. J., Sibold J., Schütte O. M., Steinem C. \& Werz D. B. $\mathrm{Gb}_{3}$ Glycosphingolipids with Fluorescent Oligoene Fatty Acids: Synthesis and Phase Behavior in Model Membranes. ChemBioChem 18, 2171-2178 (2017).

2017 Schwamborn M., Schumacher J., Sibold J., Teiwes N. K. \& Steinem C. Monitoring ATPase induced pH changes in single proteoliposomes with the lipid-coupled fluorophore Oregon Green 488. Analyst 142, 2670-2677 (2017).

\section{Oral presentations}

2017 CRC803 Autumnschool, Duderstadt

Diffusion of lipids and lipid domains in PSMs and the influence of STxB binding. 


\section{Posters}

CRC 803 Symposium 2018, Göttingen, Germany

Phase separation of sphingomyelin containing lipid bilayers is controlled by lipid-substrate adhesion as well as chain length and saturation

2018 Biennial Meeting of the German Biophysical Society 2018, Düsseldorf, Germany

Phase separation of sphingomyelin containing lipid bilayers is controlled by lipid-substrate adhesion as well as chain length and saturation

19th IUPAB and 11th EBSA congress in Edinburgh, UK

Pore spanning membranes as a versatile biomimetic tool to study phase separation and lipid diffusion

Biomembrane Days, Berlin, Germany

Lipid diffusion of a biomimetic system

2016 Biological Dynamics from Microscopic to Mesoscopic Scales, Grimma, Germany

Lipid diffusion of a biomimietic system

\section{Internship}

08/2008 German Institute of Human Nutrition Postsdam-Rehbruecke, Germany 



\section{Danksagung}

Prof. Dr. Claudia Steinem möchte ich für die Möglichkeit diese Arbeit zu gestalten danken. Ich danke dir auch dafür, dass du es immer geschafft hast, meinen Blick wieder auf den Fokus zu richten, wenn ich mich wieder an Kleinigkeiten aufgehalten habe.

Prof. Dr. Jörg Enderlein danke ich für die Übernahme des zweit Gutachtens und die fachlichen Diskussionen in den Thesis Committees.

Besonders möchte ich Prof. Dr. Daniel B. Werz, Dr. Lukas Patalag und Dr. Katharina Kettelhoit für die Synthese zahlreicher $\mathrm{Gb}_{3} \mathrm{~S}$ danken. Dr. Susann Spindler, Reza Gholami Mahmoodabadi und Prof. Dr. Vahid Sandoghdar möchte ich für die iSCAT Messungen danken. Für die Isolation von STxB möchte ich Mathias Bosse und Prof. Dr. Daniel Huster danken.

In diesem Atemzug möchte ich mich auch bei den Masterstudenten Vera Tewaag und Thomas Vagedes, den Praktikanten Julian Meyer, Thomas Vagedes und Fangyuan Liu, sowie der wissenschaftlichen Hilfkraft Loan Vuong für eure Experimente und Datenanalyse bedanken.

Ein besonderer Dank für die zahlreiche Unterstüzung im Laboralltag geht an Jutta Gerber-Nolte und Michaela Klingebiel. Danke für die unermüdliche Herstellung verschiedenster Vesikel.

Dem allerbesten Sekreteriat, Melanie Sorhage, Marianne Wagener und Dana Sachs, möchte ich für die Unterstüzung in den vielen organisatorischen Fragen danken, ich weiss, dass ihr das hier lest.

Natürlich danke ich meiner Familie für die Tatkräftige Unterstützung aus dem Schwarzwald. In der näheren Umgebung möchte ich Rebecca dafür danken, dass du immer für mich da warst. 
Tja wer umblättert hat einen Vorteil.

Dem gesammten Arbeitskreis möchte ich durch eine verschlüsselte Nachricht danken: GrosserBuchstabenSalat='olBölüjTsDANKEdenBas MdftaFÜR zAdusDIE Bs SCHÖNEasd kljZEITft \rsdnasdepyxickjrnewrL';fprintf(GrosserBuchstabenSalat([18 $1617346031252122118823236165]$ )); fprintf(GrosserBuchstabenSalat([10 48 785216512466220211318177795225346230516979 17]));

DrIngo danken ich nur für die vielen wissenschaftlichen Diskussionen über MATLAB (Spoiler für den Arbeitskreis, vielleicht hilf es euch) und meine Forschung. Ach was solls, natürlich danke ich dir auch für die sonstigen Diskussionen und witzige Augenblicke.

Für die Studienzeit in Göttingen danke ich meinen Kommilitonen und adH. 